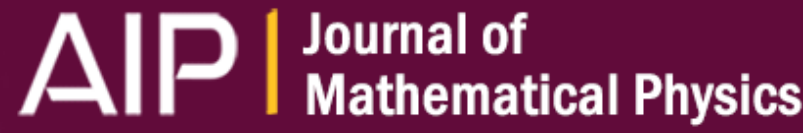

\section{Scale-invariant gauge theories of gravity: Theoretical foundations}

A. N. Lasenby and M. P. Hobson

Citation: Journal of Mathematical Physics 57, 092505 (2016); doi: 10.1063/1.4963143

View online: http://dx.doi.org/10.1063/1.4963143

View Table of Contents: http://scitation.aip.org/content/aip/journal/jmp/57/9?ver=pdfcov

Published by the AIP Publishing

\section{Articles you may be interested in}

Scale-invariant curvature fluctuations from an extended semiclassical gravity

J. Math. Phys. 56, 022303 (2015); 10.1063/1.4908127

Dirac matrices for Chern-Simons gravity

AIP Conf. Proc. 1471, 118 (2012); 10.1063/1.4756824

Non-Abelian gauge field theory in scale relativity

J. Math. Phys. 47, 032303 (2006); 10.1063/1.2176915

Birkhoff's theorem in Lovelock gravity

J. Math. Phys. 46, 072502 (2005); 10.1063/1.1960798

Gauge principle revisited: Towards a unification of space-time and internal gauge interactions J. Math. Phys. 44, 5166 (2003); 10.1063/1.1604183

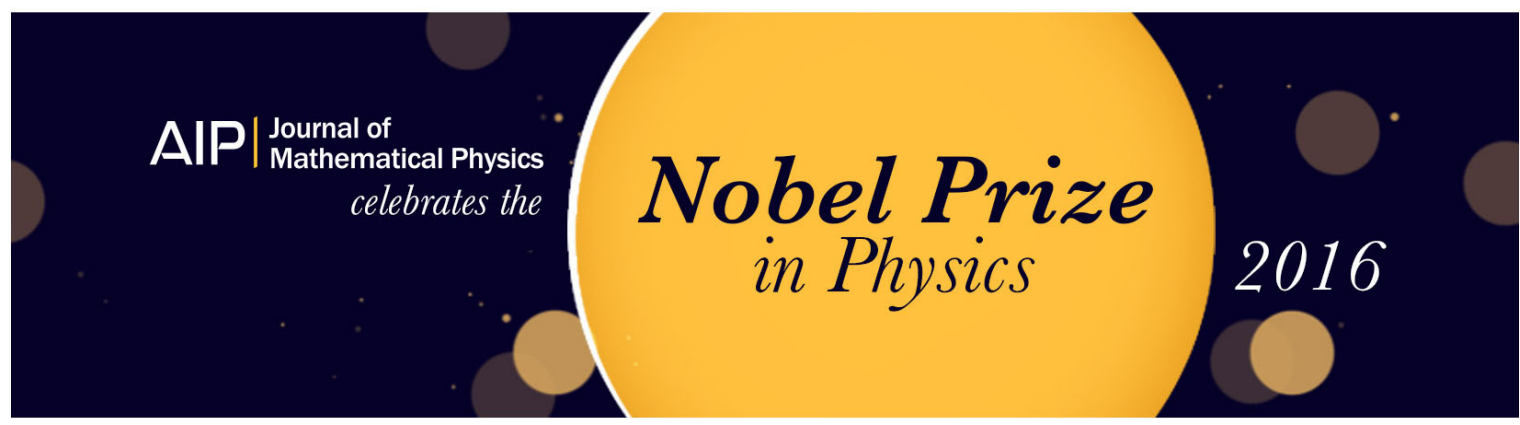




\title{
Scale-invariant gauge theories of gravity: Theoretical foundations
}

\author{
A. N. Lasenby ${ }^{1,2, a)}$ and M. P. Hobson ${ }^{1, b)}$ \\ ${ }^{1}$ Astrophysics Group, Cavendish Laboratory, JJ Thomson Avenue, \\ Cambridge CB3 OHE, United Kingdom \\ ${ }^{2}$ Kavli Institute for Cosmology, Madingley Road, Cambridge CB3 OHA, United Kingdom
}

(Received 22 October 2015; accepted 7 September 2016; published online 26 September 2016)

\begin{abstract}
We consider the construction of gauge theories of gravity, focussing in particular on the extension of local Poincaré invariance to include invariance under local changes of scale. We work exclusively in terms of finite transformations, which allow for a more transparent interpretation of such theories in terms of gauge fields in Minkowski spacetime. Our approach therefore differs from the usual geometrical description of locally scale-invariant Poincaré gauge theory (PGT) and Weyl gauge theory (WGT) in terms of Riemann-Cartan and Weyl-Cartan spacetimes, respectively. In particular, we reconsider the interpretation of the Einstein gauge and also the equations of motion of matter fields and test particles in these theories. Inspired by the observation that the PGT and WGT matter actions for the Dirac field and electromagnetic field have more general invariance properties than those imposed by construction, we go on to present a novel alternative to WGT by considering an "extended" form for the transformation law of the rotational gauge field under local dilations, which includes its "normal" transformation law in WGT as a special case. The resulting "extended" Weyl gauge theory (eWGT) has a number of interesting features that we describe in detail. In particular, we present a new scale-invariant gauge theory of gravity that accommodates ordinary matter and is defined by the most general parity-invariant eWGT Lagrangian that is at most quadratic in the eWGT field strengths, and we derive its field equations. We also consider the construction of PGTs that are invariant under local dilations assuming either the "normal" or "extended" transformation law for the rotational gauge field, but show that they are special cases of WGT and eWGT, respectively. Published by AIP Publishing. [http://dx.doi.org/10.1063/1.4963143]
\end{abstract}

\section{INTRODUCTION}

In providing a unified framework for the description of fundamental interactions, the gauge principle has proved very successful. The electromagnetic, weak, and strong interactions are all described by gauge theories, and so it is natural to consider the gauge group description of gravitation. In the gravitational case, however, in place of gauging an internal compact semi-simple Lie symmetry group, one must apply the gauge principle to spacetime symmetries, which act on spacetime coordinates as well as on the dynamical fields.

All experiments to date show that the non-gravitational fundamental interactions are consistent with the underlying spacetime symmetry of the Poincare group, and so in attempting to develop a gauge approach to gravitation, it is most natural to begin by constructing a physical theory invariant under local Poincaré transformations. As in the gauging of internal symmetries, this process requires the introduction of new, compensating gauge fields, which represent gravitational interactions.

\footnotetext{
a)Electronic mail: a.n.lasenby@mrao.cam.ac.uk

b) Electronic mail: mph@mrao.cam.ac.uk
} 
Following an initial attempt by Utiyama, ${ }^{1}$ the idea of gauging the Poincaré group was fully developed by Kibble ${ }^{2}$ (and also considered by Sciama ${ }^{3}$ ). The physical model envisaged in Kibble's approach is an underlying Minkowski spacetime in which a matter field (or fields) $\varphi$ with energy-momentum and, in general, spin-angular-momentum is distributed continuously. The field dynamics are described by a matter action $S_{\mathrm{M}}=\int L_{\mathrm{M}}\left(\varphi, \partial_{\mu} \varphi\right) d^{4} x$ that is invariant under global Poincare transformations. By then demanding the matter action be invariant with respect to (infinitesimal, passively interpreted) local Poincaré transformations, in which the ten Poincaré group parameters become arbitrary functions of position, one is led to the introduction of the new field variables $h_{a}{ }^{\mu}$ and $A^{a b}{ }_{\mu}$, which are interpreted as gravitational gauge fields corresponding, respectively, to the translational and (Lorentz) rotational parts of the Poincaré group. To construct a matter action that is invariant under local Poincaré transformations, the gravitational gauge fields $h_{a}{ }^{\mu}$ and $A^{a b}{ }_{\mu}$ are used to assemble a covariant derivative $\mathcal{D}_{a} \varphi$, and the matter action in the presence of gravity is typically obtained by the minimal coupling procedure of replacing partial derivatives in the special-relativistic matter Lagrangian by covariant ones, to obtain $S_{\mathrm{M}}=\int h^{-1} L_{\mathrm{M}}\left(\varphi, \mathcal{D}_{a} \varphi\right) d^{4} x$ (where the factor containing $h \equiv \operatorname{det}\left(h_{a}{ }^{\mu}\right)$ is required to make the integrand a scalar density rather than a scalar). As for any gauge theory, the form of the covariant derivative defines its structure, and the result in this case is known as Poincaré gauge theory (PGT). Kibble's approach improved considerably on the earlier work of Utiyama, who introduced the fields $A^{a b}{ }_{\mu}$ by localising just the Lorentz symmetry, while the $h_{a}{ }^{\mu}$ were treated as given functions of spacetime position (although at a later stage these functions were regarded as dynamical variables).

In addition to the matter action, the total action must also contain terms describing the dynamics of the free gravitational gauge fields. Indeed, the choice of these terms defines the precise form of Poincaré gauge theory under consideration. Following the normal procedure used in gauging internal symmetries, Kibble first constructed covariant field strength tensors for the gauge fields by commuting covariant derivatives, i.e., by considering $\left[\mathcal{D}_{a}, \mathcal{D}_{b}\right] \varphi$. This procedure yields two field strength tensors: $\mathcal{R}^{a b}{ }_{c d}(h, A, \partial A)$ corresponding to the gauge field $A^{a b}{ }_{\mu}$ arising from the (Lorentz) rotational part of the Poincaré group and $\mathcal{T}^{a}{ }_{b c}(h, \partial h, A)$ corresponding to the gauge field $h_{a}{ }^{\mu}$ arising from the translational part, where (suppressing indices for brevity) we have indicated the functional dependence of each field strength tensor on the gauge fields and their derivatives. ${ }^{4}$ It is worth noting that each field strength tensor is linear in the derivatives of the gauge field to which it corresponds. The free gravitational action then takes the form $S_{\mathrm{G}}=\int h^{-1} L_{\mathrm{G}}\left(\mathcal{R}^{a b}{ }_{c d}, \mathcal{T}^{a}{ }_{b c}\right) d^{4} x$, where the Lagrangian $L_{\mathrm{G}}$ is a scalar depending on the two field strengths. The total action is taken as the sum of the matter and gravitational actions, and variation of the total action with respect to the gauge fields $h_{a}{ }^{\mu}$ and $A^{a b}{ }_{\mu}$ leads to two coupled gravitational field equations in which the energy-momentum $\tau^{k}{ }_{\mu} \equiv \delta \mathcal{L}_{\mathrm{M}} / \delta h_{a}{ }^{\mu}$ and spin-angular-momentum $\sigma_{a b}{ }^{\mu} \equiv \delta \mathcal{L}_{\mathrm{M}} / \delta A^{a b}{ }_{\mu}$ of the matter field act as sources, where $\mathcal{L}_{\mathrm{M}} \equiv h^{-1} L_{\mathrm{M}}\left(\varphi, \mathcal{D}_{a} \varphi\right)$. In contrast to standard Yang-Mills gauge theory, in PGT one can construct an invariant for use in $L_{\mathrm{G}}$ that is linear in the derivatives of the gauge fields, namely, $\mathcal{R} \equiv \mathcal{R}^{a b}{ }_{a b}$. Indeed, Kibble chose simply to set $L_{\mathrm{G}} \propto \mathcal{R}$, which defines the so-called Einstein-Cartan (EC) theory, which, when re-interpreted geometrically, is a direct generalisation of general relativity to include torsion sourced by the spin-angular-momentum (if any) of the matter field.

Following Kibble's work, several other approaches to gauging the Poincaré group have been proposed, although they are all closely related to his original derivation. For example, Kibble's passive interpretation of the transformations has been criticised by Hehl et al., ${ }^{5}$ who reproduced Kibble's derivation from the standpoint of active transformations of the matter fields. In essence, Kibble considered the "total" variation $\delta \varphi \equiv \varphi^{\prime}\left(x^{\prime}\right)-\varphi(x)$ of the matter fields under the action of a passive infinitesimal local Poincaré coordinate transformation, whereas Hehl et al. and others ${ }^{6}$ considered instead the "form" variation $\delta_{0} \varphi \equiv \varphi^{\prime}(x)-\varphi(x)$. The latter approach is closer in spirit to the gauging of internal symmetries, since the form variation allows one to realise the representation of the Poincaré group as an active transformation in the space of the matter fields $\varphi$. Nonetheless, the transformation properties of the resulting gauge fields, as well as the form of the covariant derivative, are independent of these details, so that the final structure of the theory is the same. ${ }^{7}$ Hehl et al. further proposed that, in the matter field transformation $\delta_{0} \varphi$, the partial derivative in the translational generator be replaced by a covariant one to preserve its geometric meaning after 
localisation of the Poincare symmetry. It is easily shown, however, that their new form for the variation differs from the original $\delta_{0} \varphi$ only by a local Lorentz transformation and, therefore, invariance of a matter action under one form of the variation implies invariance under the other. In fact, it is unnecessary to consider only infinitesimal transformations. Mukunda ${ }^{8}$ considered finite local passive Poincaré transformations and again arrived at a theory equivalent to Kibble's. Lasenby, Doran, and $\mathrm{Gull}^{9}$ also considered finite transformations, but interpreted actively, and arrived at a gauge theory of gravity with the same mathematical structure as Kibble's theory, although formulated in the powerful language of geometric algebra, which greatly simplifies their subsequent application of the theory to various astrophysical and cosmological scenarios. In essence, however, all the above formulations are equivalent approaches to the localisation of Poincaré symmetry. Finally, it is worth noting that the gauge approach to gravitation is naturally interpreted as a field theory in Minkowski spacetime, ${ }^{6,9}$ in the same way as the gauge field theories describing the other fundamental interactions, and this is the viewpoint that we shall adopt in this paper. It is more common, however, to reinterpret the mathematical structure of PGT geometrically, whereby $h_{a}{ }^{\mu}$ and $A^{a b}{ }_{\mu}$ are considered as the components of a vierbein system and a local spin connection, respectively, in a more general Riemann-Cartan spacetime possessing non-zero curvature and torsion. ${ }^{5}$ For a thorough, modern account of the gauge approach to gravitation, see the work of Blagojevic, ${ }^{10}$ in which many of the issues above are discussed in some detail.

Although Kibble chose the simple gravitational Lagrangian $L_{\mathrm{G}} \propto \mathcal{R}$, it is clear that numerous higher-order invariants can be constructed from the field strength tensors $\mathcal{R}^{a b}{ }_{c d}$ and $\mathcal{T}^{a}{ }_{b c}$, and their inclusion in $L_{\mathrm{G}}$ cannot be ruled out a priori. Nonetheless, if one demands that the dynamics of each gauge field must be determined by a source current which corresponds to the same gauge group generator, one is naturally led to models with a Lagrangian $L_{\mathrm{G}}$ that is quadratic in the "curvature" and "torsion." The use of terms quadratic in the field strengths also mimics conventional gauge theory. In addition, one can typically ensure coincidence (or small difference) of predictions of PGT with those of general relativity in macroscopic domains, where Einstein's theory is satisfactorily verified by observations and experiments, by adding the linear Hilbert-Einstein term. ${ }^{11,12}$ Hence, one is led to consider

$$
L_{\mathrm{G}}=-\kappa^{-1}(\Lambda+a \mathcal{R})+L_{\mathcal{R}^{2}}+\kappa^{-1} L_{\mathcal{T}^{2}},
$$

where $\kappa=8 \pi G / c^{4}$ is the Einstein's gravitational constant, $\Lambda$ is a cosmological constant, $a$ is a dimensionless free parameter (which is usually positive with the sign conventions adopted in this paper $\left.{ }^{13}\right)$, and

$L_{\mathcal{R}^{2}}=\alpha_{1} \mathcal{R}^{2}+\alpha_{2} \mathcal{R}_{a b} \mathcal{R}^{a b}+\alpha_{3} \mathcal{R}_{a b} \mathcal{R}^{b a}+\alpha_{4} \mathcal{R}_{a b c d} \mathcal{R}^{a b c d}+\alpha_{5} \mathcal{R}_{a b c d} \mathcal{R}^{a c b d}+\alpha_{6} \mathcal{R}_{a b c d} \mathcal{R}^{c d a b}$,

$L_{\mathcal{T}^{2}}=\beta_{1} \mathcal{T}_{a b c} \mathcal{T}^{a b c}+\beta_{2} \mathcal{T}_{a b c} \mathcal{T}^{b a c}+\beta_{3} \mathcal{T}_{a} \mathcal{T}^{a}$,

in which the $\alpha_{i}$ and $\beta_{i}$ are also dimensionless free parameters. Note that pseudoscalar terms have been omitted by requiring parity invariance; parity-odd terms have been investigated previously ${ }^{12,14-16}$ and may, in principle, be included in the free-gravitational Lagrangian, but we do not consider them here. Also, one may use the generalised Gauss-Bonnet identity to set one of $\alpha_{1}, \alpha_{3}$, or $\alpha_{6}$ to zero, without loss of generality. We have written $L_{\mathrm{G}}$ in the form (1) so that, in natural units ( $c=\hbar=1$, which we will adopt throughout), it has dimensions of (length) ${ }^{-4}$, and hence the corresponding action $S_{\mathrm{G}}$ is dimensionless; note that in natural units $\kappa$ has dimensions of (length) ${ }^{2}$ and $\Lambda$ has dimensions of (length) ${ }^{-2}$.

In general, the equations of motion resulting from (1) contain derivatives of the gauge fields of no higher than second order. Indeed, restricting the Lagrangian to be at most quadratic in the field strengths, and hence at most quadratic in the first derivatives of the gauge fields, ensures that one satisfies the so-called pseudolinearity hypothesis, ${ }^{17}$ which suggests that the field equations should be linear in the highest (i.e., second) order derivatives of the gauge fields. ${ }^{18}$ Most importantly, the absence from the equations of motion of higher-order time derivatives of the fields ensures that such theories do not suffer from Ostrogradsky's instability, ${ }^{19,20}$ wherein the corresponding Hamiltonian is not bounded from below, so the energy of the system can take an arbitrarily negative value. While such a system for a free theory is not pathological, when it is coupled to a normal (matter) system with positive energy, the total system can "evaporate" into excitations of positive 
and negative energy degrees of freedom. Ostrogradsky's instability is a quite generic feature of higher-derivative theories and may explain why nature is described by theories having second-order equations of motion. We note that, although there are 40 dynamical variables $h_{a}{ }^{\mu}$ (or, equivalently, the components $b^{a}{ }_{\mu}$ of the inverse $h$-field) and $A^{a b}{ }_{\mu}$, the "curvature" and "torsion" field strengths are defined in terms of their antisymmetric derivatives and so do not depend on the time derivatives of $b^{a}{ }_{0}$ and $A^{a b}{ }_{0}$; thus 10 of the field equations represent constraints on initial data. Among the remaining 30 variables $b^{a}{ }_{\alpha}$ and $A^{a b}{ }_{\alpha}(\alpha=1,2,3)$, one can impose 10 gauge conditions by fixing the 10 parameters of the local Poincaré symmetry, reducing the number of independent variables to 20.

The large number of free parameters in $L_{\mathrm{G}}$ clearly offers many possibilities for constructing PGTs. For example, one of the simplest generalizations of EC theory is the so-called $\mathcal{R}+\mathcal{T}^{2}$ theory, for which $L_{\mathrm{G}}=\kappa^{-1}\left(a \mathcal{R}+L_{\mathcal{T}^{2}}\right)$. Like EC theory, however, this generalisation does not contain a kinetic part for the rotational gauge field $A^{a b}{ }_{\mu}$, so that the equations of motion couple the translation gauge field strength $\mathcal{T}^{a} b c$ (or the torsion in the geometrical reinterpretation of PGT) algebraically to the spin density of the matter field; hence the "torsion" is a non-propagating field, i.e., it must vanish outside of any matter sources. In a dynamical sense, therefore, EC and $\mathcal{R}+\mathcal{T}^{2}$ theories are incomplete: the rotational gauge field $A^{a b}{ }_{\mu}$ is introduced as an independent field, but the dynamics imposes an algebraic relation between the $A$-field and the matter spin-density. The $A$-field becomes fully dynamical only through the quadratic "curvature" terms in $L_{\mathcal{R}^{2}}$, in which case the "torsion" becomes a propagating field. Consequently, a number of $\mathcal{R}+\mathcal{R}^{2}$-type theories have been investigated, as have $\mathcal{R}^{2}+\mathcal{T}^{2}$ theories. ${ }^{21}$ It is worth mentioning that the latter allow the possibility of repulsive torsion interactions at small distances, which may prevent gravitational collapse to infinite matter densities. ${ }^{22}$ A summary of the properties of general $\mathcal{R}+\mathcal{R}^{2}+\mathcal{T}^{2}$ theories is given by Obukhov et al. ${ }^{12}$ The properties of even more general actions, and the coupling to matter fields, have been discussed. ${ }^{23}$ In broad terms, if $L_{\mathrm{G}}$ consists of $\mathcal{R}$ and $\mathcal{T}^{2}$ terms, then it contains dimensional constants, which is not an attractive property for quantisation. On the other hand, the $\mathcal{R}^{2}$ terms contain dimensionless constants, but the classical limit of theories without the linear curvature term $\mathcal{R}$ is questionable. ${ }^{10}$

Despite the advantages of the above framework for constructing $L_{\mathrm{G}}$, certain general difficulties do arise that go beyond merely the paucity of guiding physical principles for elucidating the magnitude or origin of the many free parameters in $L_{\mathrm{G}}$, or the algebraic complexity of the resulting field equations. For example, although the field equations that follow from the variational principle are of second differential order, if one substitutes for the rotational gauge field $A^{a b}{ }_{\mu}$ in terms of $h_{a}{ }^{\mu}$, one can rewrite them in a form that contains third-order derivatives, and it becomes difficult to assess whether they are well-posed. ${ }^{24} \mathrm{~A}$ judicious choice of the parameters in $L_{\mathrm{G}}$ can eliminate such higher derivatives from the theory, ${ }^{25}$ although the resulting theories are not without their difficulties, such as the lack of a sensible Newtonian limit or well-defined initial value problem. ${ }^{26}$ Nonetheless, the general structure of the equations of motion has been analysed to clarify the evolution of given initial data, i.e., the Cauchy problem. Of the 40 equations of motion, it is found that 10 represent constraints on the initial data, as mentioned above, and the remaining 30 define a consistent time evolution of the dynamical variables provided that the parameters $a, \alpha_{i}$, and $\beta_{i}$ in the Lagrangian $L_{\mathrm{G}}$ obey certain conditions. ${ }^{27}$

In assessing various PGTs as candidate theories of gravitation, however, the issues of greatest interest are renormalisability and unitarity, both of which can be investigated by examining the particle content of the theory. This is performed by first linearizing the 40 gauge fields $h_{a}^{\mu}$ and $A^{a b}{ }_{\mu}$ about the trivial vacuum solution to the field equations and retaining only those terms in the Lagrangian $L_{\mathrm{G}}$ that are bilinear in the linearized gauge fields to obtain $L_{\mathrm{G}}^{(2)}$. After transforming to momentum space, one chooses covariant basis states, so that $L_{\mathrm{G}}^{(2)}$, when considered as a $40 \times 40$-matrix in field space, becomes mostly zeros. The covariant basis states are constructed using $k$-space projection operators, ${ }^{28}$ which decompose the linearised gauge fields into tensors that reduce to states of definite spin-parity $J^{P}$ in a "rest-frame" where $k^{a}$ has only a time component and are thus irreducible representations of the Poincare group. One finds that of the 20 independent degrees of freedom in the gauge fields, two correspond to the massless graviton (with 
spin-parity $\left.J^{P}=2^{+}\right)$and the decomposition of $A^{a b}{ }_{\alpha}(\alpha=1,2,3)$ yields (in general, massive) tordions (or rotons) with spin-parities of $J^{P}=2^{ \pm}, 1^{ \pm}, 0^{ \pm}$, with $2(5+3+1)=18$ degrees of freedom. The propagator is simply (minus) the inverse of the terms in the resulting bilinear Lagrangian. ${ }^{29}$

For a theory to be unitary, its particle spectrum must be free from ghosts (particles with negative definite free-field energy, which destroys the unitarity of the $S$ matrix) and tachyons (particles which propagate faster than light and therefore violate causality). One can check for ghosts or tachyons by examining the residues and locations of the propagator poles, which generally depend in a complicated way on all the parameters $a, \alpha_{i}$, and $\beta_{i}$ in the original Lagrangian $L_{\mathrm{G}}$. The condition for no tachyons is that the propagator has first-order poles for non-negative $k^{2}$, whereas the condition for no ghosts is that the matrix of the residues at the poles is positive definite. In other words, each element of the diagonalized propagator must be of the form $-R /\left(k^{2}-m^{2}\right)$ with $R>0$ and $m^{2} \geq 0$ (assuming a "mostly minus" metric signature). These requirements follow from the spectral representation of the vacuum expectation value of the time-ordered product. ${ }^{30}$ In PGT, for the general case in which all the tordion modes are massive, there exist ranges of values of the parameters in $L_{\mathrm{G}}$ (that divide into 5 broad classes) for which the resulting PGT is indeed unitary. ${ }^{31}$ If one further requires that the torsion propagates, one finds that there are 12 six-parameter Lagrangians that lead to unitary theories. ${ }^{32}$ For the special case in which there are massless tordion modes, and hence the possibility of long-range torsion effects, it is important to take into account the transversality relations between residues (for example, they are needed to eliminate ghosts from general relativity). In this case, the results are not so complete. ${ }^{18,33}$ Nonetheless, at least one 3-parameter Lagrangian exists that propagates (only) a massless $J^{P}=1^{-}$tordion and results in a unitary theory. ${ }^{32}$ It is worth noting that a generic feature of massless tordion Lagrangians is the appearance of extra gauge symmetries. The introduction of a scalar field allows for a still larger choice of unitary Lagrangians. ${ }^{34}$

For a theory to be power-counting renormalisable, one requires good high-energy behaviour of the propagators for its constituent particles. In particular, in all spin sectors, the propagators for the graviton should behave as $\sim 1 / k^{4}$, whereas those for the tordions should be $\sim 1 / k^{2}$, since tordion vertices are better behaved than those of gravitons. ${ }^{29,31,35}$ Pure $1 / k^{4}$ poles can occur in PGT, but are forbidden since they give rise to field modes with free-field energies not bounded from below ("dipole-ghosts"). ${ }^{36}$ Nonetheless, the PGT propagator also has pole structures of the form $\left(k^{2}-m_{1}^{2}\right)^{-1}\left(k^{2}-m_{2}^{2}\right)^{-1}=\left(m_{2}^{2}-m_{1}^{2}\right)^{-1}\left[\left(k^{2}-m_{2}^{2}\right)^{-1}-\left(k^{2}-m_{1}^{2}\right)^{-1}\right]$. These can be tolerated in lower spin-sectors, since the resulting ghost mode may be compensated by lower-spin terms in the higher-spin projection operators. This cannot occur in the spin-2 sector, however, and so any powercounting renormalizable PGT will have ghosts in the spin-2 sector. ${ }^{37}$ Conversely, to remove the ghosts, the $\sim 1 / k^{4}$ propagators must cancel, making the theory power-counting non-renormalizable. Indeed, for unitary massive-tordion Lagrangians, the graviton propagator goes as $\sim 1 / k^{2}$ and the tordion propagator tends to a constant at high energies. For the massless propagating $J^{P}=1^{-}$ tordion Lagrangian, however, the tordion propagator goes as $\sim 1 / k^{2}$, leading to improved ultraviolet behaviour. ${ }^{32}$ Although power-counting renormalizable PGTs are not unitary, and vice versa, it still remains possible that unitary PGTs are renormalizable when higher-order loops are taken into account, but relatively little is known. It has been shown ${ }^{38}$ that, in the absence of torsion, $\mathcal{R}+\mathcal{R}^{2}$ theories are renormalizable (but not unitary). The renormalisability of EC theory and the quantum effect of quadratic torsion terms at one-loop level have been investigated. ${ }^{6,39}$ More innovative approaches to quantisation of PGT have been pursued, ${ }^{40,41}$ but are also problematic. ${ }^{42}$

The lack of a clear route to quantising PGT has led to interest in imposing extra gauge symmetries beyond local Poincaré symmetry, since this could, in principle, lead to surprises that might allow for a simultaneously unitary and renormalizable theory, ${ }^{31,43}$ without having to resort to non-local theories. ${ }^{44-46}$ In particular, perhaps the most natural extension of PGT is also to demand local scale invariance, which might resolve some of the problems outlined above, ${ }^{47}$ since such theories contain no absolute energy scale. It should be noted, however, that scale invariance defines an unacceptable transformation law for particle masses. If scale invariance were to hold in nature, it would imply that the mass spectrum is either continuous (if $m^{2} \neq 0$ ) or all the masses vanish. Thus scale invariance must be a broken symmetry in a world with non-vanishing, discrete particle masses. 
The most direct approach to constructing gauge theories of gravity that are invariant under local changes of scale, in addition to local Poincaré transformations, is to gauge the Weyl group W(1,3), which extends Poincaré symmetry to include scale invariance and is a sub-group of the full conformal group $\mathrm{C}(1,3) \cdot{ }^{48-50}$ This may be formulated in a number of ways, e.g., by considering the Weyl transformations as active or passive, infinitesimal or finite, but they are all essentially equivalent. As in PGT, one assumes the physical model of an underlying Minkowski spacetime in which a continuum matter field (or fields) $\varphi$ with energy-momentum and, in general, spin-angular-momentum is distributed continuously. Now, however, the field dynamics are described by a matter action $S_{\mathrm{M}}=\int L_{\mathrm{M}}\left(\varphi, \partial_{\mu} \varphi\right) d^{4} x$ that is invariant under global Weyl coordinate transformations, which imposes much more severe constraints on the form of the action. By then demanding the matter action to be invariant with respect to local Weyl transformations, in which the eleven Weyl group parameters become arbitrary functions of position, one is again led to the introduction of gauge fields $h_{a}{ }^{\mu}$ and $A^{a b}{ }_{\mu}$ (although the transformation rules of the former are different to those in PGT) and also a new gravitational gauge field $B_{\mu}$ corresponding to the dilation part of the Weyl group. ${ }^{51-53}$ Similar to PGT, the gravitational gauge fields $h_{a}{ }^{\mu}, A^{a b}{ }_{\mu}$, and $B_{\mu}$ are used to assemble a covariant derivative $\mathcal{D}_{a}^{*} \varphi$, and the matter action in the presence of gravity is obtained by the minimal coupling procedure, so that $S_{\mathrm{M}}=\int h^{-1} L_{\mathrm{M}}\left(\varphi, \mathcal{D}_{a}^{*} \varphi\right) d^{4} x$. It should be noted, however, that the requirement of local scale invariance imposes tight constraints on the precise form of $L_{\mathrm{M}}$. In particular, since $h^{-1}$ has a Weyl (or conformal) weight $w\left(h^{-1}\right)=4$ (see Section II B), the Lagrangian $L_{\mathrm{M}}$ must have a weight $w\left(L_{\mathrm{G}}\right)=-4$.

As in PGT, the action term describing the dynamics of the free gravitational gauge fields is assembled by first constructing covariant field strength tensors for the gauge fields by commuting covariant derivatives, i.e., by considering $\left[\mathcal{D}_{a}^{*}, \mathcal{D}_{b}^{*}\right] \varphi$. One obtains $\mathcal{R}^{a b}{ }_{c d}(h, A, \partial A)$ and $\mathcal{T}^{* a}{ }_{b c}(h, \partial h, A, B)$ as before (but where the form of the latter differs from that in PGT) and an additional field strength $\mathcal{H}_{a b}(h, \partial B)$ corresponding to the new gauge field $B_{\mu}$ arising from the dilation part of the Weyl group (we have again indicated the functional dependence of each field strength tensor on the gauge fields and their derivatives, suppressing indices for brevity). As in PGT, each field strength tensor is linear in the derivatives of the gauge field to which it corresponds. The free gravitational action then has the general form $S_{\mathrm{G}}=\int h^{-1} L_{\mathrm{G}}\left(\mathcal{R}^{a b}{ }_{c d}, \mathcal{T}^{* a}{ }_{b c}, \mathcal{H}_{a b}\right) d^{4} x$. As usual, the total action is taken as the sum of the matter and gravitational actions, and variation of the total action with respect to the gauge fields $h_{a}{ }^{\mu}, A^{a b}{ }_{\mu}$, and $B_{\mu}$ leads to three coupled gravitational field equations in which the energy-momentum $\tau^{k}{ }_{\mu} \equiv \delta \mathcal{L}_{\mathrm{M}} / \delta h_{a}{ }^{\mu}$, spin-angular-momentum $\sigma_{a b}{ }^{\mu} \equiv \delta \mathcal{L}_{\mathrm{M}} / \delta A^{a b}{ }_{\mu}$, and dilation current $\zeta^{\mu} \equiv \delta \mathcal{L}_{\mathrm{M}} / \delta B_{\mu}$ of the matter field act as sources.

The free gravitational Lagrangian $L_{\mathrm{G}}$ must also have a Weyl (conformal) weight $w\left(L_{\mathrm{G}}\right)=-4$, which places tight constraints on its form. It is easily shown that $w\left(\mathcal{R}^{a b}{ }_{c d}\right)=w\left(\mathcal{H}_{a b}\right)=-2$ and $w\left(\mathcal{T}^{* a}{ }_{b c}\right)=-1$, which means that $L_{\mathrm{G}}$ can be quadratic in $\mathcal{R}^{a b}{ }_{c d}$ and $\mathcal{H}_{a b}$, while terms linear in $\mathcal{R} \equiv \mathcal{R}^{a b}{ }_{a b}$ or quadratic in $\mathcal{T}^{* a}{ }_{b c}$ are not allowed, despite them transforming covariantly under local Weyl transformations. Thus, in Weyl gauge theory (WGT), the general form of $L_{\mathrm{G}}$, possessing terms no higher than quadratic order in the field strengths, and hence at most quadratic in the first derivatives of gauge fields, is of $\mathcal{R}^{2}+\mathcal{H}^{2}$ type,

$$
\begin{aligned}
L_{\mathrm{G}}= & \alpha_{1} \mathcal{R}^{2}+\alpha_{2} \mathcal{R}_{a b} \mathcal{R}^{a b}+\alpha_{3} \mathcal{R}_{a b} \mathcal{R}^{b a}+\alpha_{4} \mathcal{R}_{a b c d} \mathcal{R}^{a b c d}+\alpha_{5} \mathcal{R}_{a b c d} \mathcal{R}^{a c b d}+\alpha_{6} \mathcal{R}_{a b c d} \mathcal{R}^{c d a b} \\
& +\xi \mathcal{H}_{a b} \mathcal{H}^{a b} \equiv L_{\mathcal{R}^{2}}+L_{\mathcal{H}^{2}},
\end{aligned}
$$

where the $\alpha_{i}$ and $\xi$ are dimensionless free parameters. Once again pseudo-scalar terms have been omitted by requiring parity invariance, and one may use the generalised Gauss-Bonnet identity to set one of $\alpha_{1}, \alpha_{3}$, or $\alpha_{6}$ to zero, without loss of generality. Evidently, local Weyl invariance removes many of the possibilities that exist in PGT; essentially the $\mathcal{R}$ and $\mathcal{T}^{* 2}$ terms, which possess dimensional constants, are forbidden.

Although the requirement that each term in the total Lagrangian must have a Weyl weight $w=$ -4 clearly places quite restrictive conditions on its form, one can construct further Weyl-covariant terms with the appropriate weight for inclusion in the Lagrangian by introducing an additional massless scalar field (or fields) $\phi$ with Weyl weight $w(\phi)=-1$, often termed the compensator(s). ${ }^{10}$ This opens up possibilities for the inclusion of further action terms in which the scalar field is non-minimally (conformally) coupled to the field strength tensors of the gravitational gauge fields, 
combined (usually) with an additional free kinetic term for $\phi$. For example, terms proportional to $\phi^{2} \mathcal{R}$ or $\phi^{2} L_{\mathcal{T} * 2}$ are Weyl-covariant with weight $w=-4$ and so may be added to the total Lagrangian. The inclusion of the term $\phi^{2} \mathcal{R}$ has been widely investigated, ${ }^{54,55}$ whereas the phenomenological value of the $\phi^{2} L_{\mathcal{T} * 2}$ terms has received relatively little attention. ${ }^{56,57}$ In any case, the resulting total Lagrangian is at most quadratic in the first derivatives of the gauge fields, thereby satisfying the pseudolinearity hypothesis that field equations be linear in the second-order derivatives of the gauge field, and hence ensuring that such theories do not suffer from Ostrogradsky's instability.

The inclusion of scalar fields also allows for more flexibility in the allowed forms of the matter energy-momentum tensor. Let us take as an example the action for a free Dirac field $\psi$, which has Weyl weight $w(\psi)=w(\bar{\psi})=-\frac{3}{2}$. This action is not scale-invariant owing to the mass term $m \bar{\psi} \psi$, so it appears that one requires the field to be massless, which clearly cannot describe "ordinary" matter. One may reach the same conclusion by noting that in WGT the trace of the total matter energy-momentum tensor must equal the covariant divergence of the dilation current ${ }^{10}$ $\zeta^{\mu} \equiv \delta \mathcal{L}_{\mathrm{M}} / \delta B_{\mu}$. In the case of Dirac matter, the Lagrangian resulting from gauging the Weyl symmetry, curiously, does not contain the dilation gauge field $B_{\mu}$. Hence, for Dirac matter on its own, the energy-momentum tensor of $\psi$ must be traceless, which again requires the field to be massless. These difficulties can be circumvented, however, by making the replacement $m \bar{\psi} \psi \rightarrow \mu \phi \bar{\psi} \psi$ in the Dirac action, where $\mu$ is a dimensionless parameter but $\mu \phi$ has the dimensions of mass in natural units. Although the trace of the total energy momentum tensor of the $\psi$ and $\phi$ fields must still vanish, the energy-momentum tensor of the Dirac matter field $\psi$ itself need not be traceless, thereby allowing it to be massive. Indeed, this approach to the construction of gauge theories of gravity that are scale-invariant but, at the same time, are able to accommodate "ordinary" matter was first explored by Dirac ${ }^{54}$ in the context of attempting to establish a deeper physical understanding of his "large numbers hypothesis" relating microscopic (quantum) and macroscopic (gravitational) scales. In particular, Dirac hoped that the additional scalar field $\phi$ might provide a time-dependent coupling of gravitation to matter, hence allowing the Newtonian gravitational "constant" $G$ to vary with cosmic epoch. This idea can be naturally accommodated in the framework of WGT ${ }^{58}$ (and extended to include non-zero torsion, which was assumed to vanish by Dirac).

In general, the introduction of scalar matter fields in WGT, and scale-invariant theories more generally, is also important since they provide a natural means for spontaneously breaking the scale symmetry, which is necessary to achieve an acceptable long-range limit. The approach most commonly adopted is to use local scale invariance to set the compensator scalar field $\phi$ to a constant value in the resulting field equations, which is known as the Einstein gauge. Setting $\phi=\phi_{0}$ in the equation of motion for the Dirac field $\psi$, for example, leads to its interpretation as a massive field with $m=\mu \phi_{0}$. It is usually considered that setting $\phi=\phi_{0}$ represents the choice of some definite scale in the theory, thereby breaking scale-invariance. Indeed, it is often given the physical interpretation of corresponding to some spontaneous breaking of the scale symmetry (where nature chooses the gauge). As we show in Section II L, however, this interpretation is questionable. In particular, we demonstrate in WGT that the equations of motion in the Einstein gauge are identical in form to those obtained when working in scale-invariant variables, where the latter involves no breaking of the scale symmetry. This suggests that one should introduce further scalar fields, in addition to the compensator field $\phi$, to enable a true physical breaking of the scale symmetry.

In general, the phenomenology of WGT is far less well understood than that of PGT. As for PGT, the presence of $\mathcal{R}^{2}$ terms in the action improves the quantum renormalisability of such theories, but can again lead to problems with unitarity. ${ }^{10}$ These issues, as well as the stability of the ground state, have yet to be resolved, but the development of a full phenomenological basis for WGT remains of considerable theoretical interest.

In this paper, however, our primary aim is to present a novel alternative to WGT by considering a more general "extended" transformation law for the rotational gauge field under local dilations, which includes its "normal" transformation law in WGT as a special case. This is motivated by the observation that the PGT (and WGT) matter actions both for the Dirac field and the electromagnetic field are already invariant under local dilations if one assumes this "extended" transformation law, in the same way as they are for the "normal" transformation law assumed in WGT. Moreover, under a global scale transformation, the two transformation laws coincide, and so both may be considered 
as an equally valid gauging of global Weyl scale invariance. The key difference between the two sets of transformations is that, whereas under the "normal" Weyl transformations the PGT gauge fields $h_{a}{ }^{\mu}$ and $A^{a b}{ }_{\mu}$ transform covariantly with weights of $w,-1$ and 0 , respectively, under the "extended" transformations the rotational gauge field $A^{a b}{ }_{\mu}$ transforms inhomogeneously. This also has the consequence that the transformation properties of the PGT rotational gauge field strength (or "curvature") $\mathcal{R}_{a b c d}$ and translational gauge field strength (or "torsion") $\mathcal{T}_{a b c}$ are treated in a more balanced manner. The resulting "extended" Weyl gauge theory (eWGT) has a number of interesting features that we describe in detail, although we focus here only on its formal description and postpone a full discussion of its application and phenomenology to future publications.

Finally, it is worth mentioning that one can also construct gauge theories of gravity that are invariant under local changes of scale (assuming either the normal or extended transformation law for the rotational gauge field), in addition to local Poincaré transformations, without gauging the Weyl group. Instead, one works entirely in terms of PGT objects, such as matter fields $\varphi$ and their PGT covariant derivatives $\mathcal{D}_{a} \varphi$, the PGT gauge field strengths $\mathcal{R}^{a b}{ }_{c d}$ and $\mathcal{T}^{a}{ }_{b c}$ (or even their simpler counterparts in the absence of "torsion"), and a (compensator) scalar field $\phi$ (as discussed above). These are used to construct terms in the free gravitational and matter actions that individually are not necessarily invariant (or even covariant) under (extended) local scale changes, but by taking the appropriate linear combinations of such terms, it is possible to arrive at a free gravitational action and matter action that are locally scale-invariant (and hence so is the total action). ${ }^{59-62,89}$

The remainder of this paper is arranged as follows. In Section II, we establish our general approach to constructing locally scale-invariant gauge theories of gravity by giving a brief outline of the mathematical structure of WGT, but in a way not usually presented in the literature. In particular, we maintain throughout the notion of $h_{a}{ }^{\mu}, A^{a b}{ }_{\mu}$, and $B_{\mu}$ as gauge fields in Minkowski spacetime, rather than adopting the more usual geometric interpretation of WGT in terms of the curvature and torsion of a Weyl-Cartan $Y_{4}$ spacetime (although we do give a brief account of the latter); this is in keeping with the gauge field theories describing the other fundamental interactions and has some non-trivial consequences. We also work exclusively in terms of finite local Weyl transformations, rather than their usual infinitesimal forms; the former allows, in our view, for a more transparent interpretation of WGT. We also make some observations that differ from typical accounts of the subject, in particular regarding the interpretation of the Einstein gauge and also the equations of motion of matter fields and test particles. In Section III, we present our novel alternative to WGT, obtained by demanding invariance under a more general form for the transformation law of the rotational gauge field under local dilations, and describe the general properties of the resulting "extended" Weyl gauge theory (eWGT); we also discuss its interpretation in terms of a new spacetime geometry (that we term $\widehat{Y}_{4}$ ), which is an extended form of Weyl-Cartan $Y_{4}$ spacetime. In Section IV, we present a new scale-invariant gauge theory of gravity that can accommodate ordinary matter and is defined by the most general eWGT Lagrangian that is at most quadratic in the eWGT field strengths, and we derive its field equations. In Section V, we briefly discuss the construction of PGT actions that are invariant under local dilations. We conclude in Section VI. In addition, we include six appendices. Appendix A presents a re-expression of the special-relativistic Lagrangian of a Dirac field, which lends itself to the development of a semi-classical description of a spinning point particle, which we will use as our model of "ordinary" matter. Appendix B provides a brief description of PGT as a special case, with appropriate notational modifications, of our discussion of WGT in Section II, and Appendix C summarises the particular PGT originally considered by Kibble, namely, Einstein-Cartan theory. In Appendix D, as an example of a WGT, we describe the first proposed scale-invariant theory of gravity that can accommodate "ordinary" matter, which was explored by Dirac, ${ }^{54}$ but extend it to include non-zero torsion. In Appendix E, we present an alternative approach to deriving the form of the covariant derivative in eWGT, which is complementary to that presented in Section III. Finally, in Appendix F, we present a summary of our notation. The phenomenology of eWGT and its application to astrophysics and cosmology will be described in forthcoming papers. 


\section{WEYL GAUGE THEORY}

As discussed in the Introduction, the minimal and perhaps most natural extension of PGT is based on gauging the Weyl group W(1,3), which extends Poincaré symmetry to include scale invariance. Thus, in traditional Weyl gauge theory (WGT), in addition to local Poincaré symmetry, one also demands invariance under local change of scale. We give an account of the mathematical structure of WGT here. In particular, we will interpret Weyl transformations passively, but consider them in their more transparent finite form, rather than as infinitesimal transformations. The notation used broadly follows Kibble ${ }^{2}$ and Mukunda. ${ }^{8}$ It is worth noting that, with appropriate notational modifications, the following discussion also serves as an account of PGT, which may be considered as a special case of WGT, as described in Appendix B.

\section{A. Global Weyl invariance}

We begin with a Minkowski spacetime $\mathcal{M}$, labelled using Cartesian inertial coordinates $x^{\mu}$, in which the dynamics of some multiplet of "matter" (i.e., non-gravitational) fields $\varphi(x)$ is described by a matter action

$$
S_{\mathrm{M}}=\int L_{\mathrm{M}}\left(\varphi, \partial_{\mu} \varphi\right) d^{4} x,
$$

that is invariant under global Weyl transformations, which imposes much more severe constraints on the form of the action than those required by mere global Poincare invariance. We denote the coordinate basis vectors by $\boldsymbol{e}_{\mu}$, which are tangents to the coordinate curves and for Cartesian inertial coordinates satisfy $\boldsymbol{e}_{\mu} \cdot \boldsymbol{e}_{\nu}=\eta_{\mu \nu}$, where $\eta_{\mu \nu}=\operatorname{diag}(1,-1,-1,-1)$. At each point of $\mathcal{M}$, we can also define a local Lorentz reference frame, unrelated to the coordinates $x^{\mu}$, which is represented by an orthonormal tetrad $\hat{\boldsymbol{e}}_{a}(x)$, such that $\hat{\boldsymbol{e}}_{a} \cdot \hat{\boldsymbol{e}}_{b}=\eta_{a b}$. In global Cartesian inertial coordinates $x^{\mu}$, we can always choose the tetrad such that it coincides with a coordinate frame $\boldsymbol{e}_{\mu}(x)$, i.e., $\hat{\boldsymbol{e}}_{a}=\delta_{a}^{\mu} \boldsymbol{e}_{\mu}$. We will adopt the common convention that Latin indices refer to local tetrad frames, while Greek indices refer to coordinate frames.

We suppose that our multiplet of matter fields $\varphi(x)$ belongs to some representation of the Weyl group. More precisely, we replace the usual Lorentz subgroup $\operatorname{SO}(3,1)$ by $\operatorname{SL}(2, C)$, so that spinor fields can be accommodated. ${ }^{63}$ Indeed, by expressing $\varphi(x)$ in its 2 -spinor form, for example, one can always ensure that its components carry no coordinate (Greek) indices. Such an approach can be rather cumbersome, however, and so we adopt a more straightforward notation in which the matter field components $\left\{\varphi^{a}(x)\right\}$ simply carry a generic Roman index (or set of indices) to denote that they are "referred to" a local Lorentz frame. Thus, matter field components (which may be expressed in 2-spinor, 4-spinor, or 4-tensor form, as is most convenient) behave as scalars under a change of coordinates, but transform under a corresponding rotation of the local tetrad frames (such that they coincide with the new coordinate frame at each point) or physical dilations according to their $\operatorname{SL}(2, C)$ representation and Weyl weight (see below), respectively. This distinction will become important shortly, when we consider localising the Weyl symmetry.

Thus, under a global Weyl transformation

$$
x^{\prime \mu}=e^{\rho}\left(\Lambda^{\mu}{ }_{v} x^{v}+a^{\mu}\right),
$$

the multiplet of matter fields transforms via

$$
\varphi^{\prime}\left(x^{\prime}\right)=e^{w \rho} \mathrm{S}(\Lambda) \varphi(x),
$$

where $\mathrm{S}(\Lambda)$ is the matrix corresponding to the element $\Lambda$ of the Lorentz group (or SL(2,C) group) in the representation to which $\varphi(x)$ belongs (we have suppressed Lorentz indices on these objects for notational simplicity), and $w$ is the Weyl (or conformal) weight of the field $\varphi(x)$. Moreover, the derivatives of the matter fields transform as

$$
\partial_{\mu}^{\prime} \varphi^{\prime}\left(x^{\prime}\right)=e^{(w-1) \rho} \Lambda_{\mu}{ }^{\nu} \mathrm{S}(\Lambda) \partial_{\nu} \varphi(x) .
$$


It is worth briefly considering in an explicitly geometrical manner the dilation part of the Weyl transformation, i.e., with $\Lambda^{\mu}{ }_{v}=\delta_{v}^{\mu}$ and $a^{\mu}=0$ in (6) and (7). This transformation may be considered as consisting of two distinct parts. The first part physically (actively) dilates the Minkowski spacetime such that the distance between any two points is increased by a factor $e^{\rho}$. Adopting global inertial coordinates, such that $d s^{2}=\eta_{\mu \nu} d x^{\mu} d x^{\nu}$, the metric must therefore transform according to $\eta_{\mu \nu} \rightarrow \gamma_{\mu \nu}=e^{2 \rho} \eta_{\mu \nu}$. Hence, under this active dilation the metric has a Weyl weight of $w=2$. Note, however, that no coordinate transformation has occurred, since every point in the spacetime still has its original coordinate labels; one may consider the coordinates as being "embedded" in the spacetime as it dilates. The second part of the transformation consists of a coordinate transformation $x^{\prime \mu}=e^{\rho} x^{\mu}$, which "shrinks" the coordinate system back to its original "size." Under this coordinate transformation, $\gamma_{\mu \nu}^{\prime}=e^{-2 \rho} \gamma_{\mu \nu}=\eta_{\mu \nu}$ and so, overall, the metric remains unchanged. This distinction between the two parts of the Weyl dilation will become important when we localise the Weyl symmetry, at which point they decouple completely.

For invariance of the matter action under a global Weyl transformation (6) (for which the Jacobian equals $e^{4 \rho}$ ), a sufficient condition is that the matter Lagrangian density satisfies

$$
L_{\mathrm{M}}\left(\varphi^{\prime}\left(x^{\prime}\right), \partial_{\mu}^{\prime} \varphi^{\prime}\left(x^{\prime}\right)\right)=e^{-4 \rho} L_{\mathrm{M}}\left(\varphi(x), \partial_{\mu} \varphi(x)\right),
$$

where the arguments on the LHS are given by (7) and (8), respectively.

For our later purposes, it is useful to consider the concrete examples provided by the standard special-relativistic matter Lagrangians for a massive free scalar field, a massive free Dirac field, and the electromagnetic field, respectively. For a (real) free scalar field $\phi$ of mass $m$, one has the Klein-Gordon Lagrangian

$$
L_{\mathrm{KG}}=\frac{1}{2} \eta^{\mu v} \partial_{\mu} \phi \partial_{\nu} \phi-\frac{1}{2} m^{2} \phi^{2},
$$

and under the dilation part of a Weyl transformation (6) and (7), one thus obtains

$$
\begin{aligned}
L_{\mathrm{KG}}^{\prime} & =\frac{1}{2} \gamma^{\prime \mu \nu} \partial_{\mu}^{\prime} \phi^{\prime} \partial_{\nu}^{\prime} \phi^{\prime}-\frac{1}{2} m^{2} \phi^{\prime 2} \\
& =\frac{1}{2} e^{2(w-1) \rho} \eta^{\mu \nu} \partial_{\mu} \phi \partial_{\nu} \phi-\frac{1}{2} m^{2} e^{2 w \rho} \phi^{2} .
\end{aligned}
$$

For invariance of the corresponding matter action, one requires (9) to hold. Thus, the free scalar field action is dilation invariant only for $m=0$ and provided the Weyl weight of the field is taken as $w(\phi)=-1$.

One can perform a similar analysis for the Lagrangian for a classical Dirac field $\psi$ of mass $m$, which is given by ${ }^{64}$

$$
L_{\mathrm{D}}=\frac{1}{2} i\left[\bar{\psi} \gamma^{\mu} \partial_{\mu} \psi-\left(\partial_{\mu} \bar{\psi}\right) \gamma^{\mu} \psi\right]-m \bar{\psi} \psi \equiv \frac{1}{2} i \bar{\psi} \gamma^{\mu} \stackrel{\leftrightarrow}{\partial_{\mu}} \psi-m \bar{\psi} \psi
$$

where the kinetic energy term is written so as to ensure that it is always real (and the sign ensures that the terms contribute positive energy on quantisation). Under a dilation transformation, one obtains

$$
\begin{aligned}
L_{\mathrm{D}}^{\prime} & =\frac{1}{2} i \overline{\psi^{\prime}} \gamma^{\mu} \stackrel{\leftrightarrow}{\partial_{\mu}^{\prime}} \psi^{\prime}-m \bar{\psi}^{\prime} \psi^{\prime} \\
& =\frac{1}{2} i e^{(2 w-1) \rho} \bar{\psi} \gamma^{\mu} \stackrel{\leftrightarrow}{\partial_{\mu}} \psi-m e^{2 w \rho} \bar{\psi} \psi .
\end{aligned}
$$

Demanding (9) to hold, one thus finds that the Dirac action is dilation invariant only for $m=0$ and provided the Weyl weight of the Dirac field is taken as $w(\psi)=w(\bar{\psi})=-\frac{3}{2}$, which is consistent with the interpretation of $\bar{\psi} \psi$ as a number density. As mentioned in the Introduction, however, one can circumvent this restriction to a massless Dirac field by introducing a massless scalar field $\phi$ and making the replacement $m \bar{\psi} \psi \rightarrow \mu \phi \bar{\psi} \psi$ in the Dirac Lagrangian, where $\mu$ is a dimensionless parameter, combined usually with an additional kinetic term for $\phi$. One thus obtains

$$
L_{\mathrm{M}}=\frac{1}{2} i \bar{\psi} \gamma^{\mu} \stackrel{\leftrightarrow}{\partial_{\mu}} \psi+\frac{1}{2} \partial_{\mu} \phi \partial^{\mu} \phi-\mu \phi \bar{\psi} \psi
$$

which is easily shown to be invariant under a dilation transformation and describes a massless Dirac field interacting with a massless scalar field. If one then adopts the Einstein gauge $\phi=\phi_{0}$ in the resulting field equations, however, one may interpret $\psi$ as a massive field with $m=\mu \phi_{0}$. 
Finally, we consider the electromagnetic field $A_{\mu}$ (which should not be confused with the "rotational" gravitational gauge field $A^{a b}{ }_{\mu}$ ), for which the special-relativistic Lagrangian is given by

$$
L_{\mathrm{EM}}=-\frac{1}{4} F_{\mu \nu} F^{\mu \nu}-J^{\mu} A_{\mu},
$$

where $F_{\mu \nu} \equiv \partial_{\mu} A_{v}-\partial_{\nu} A_{\mu}$ and $J^{\mu}$ is some source current density. Under a dilation transformation, one thus obtains

$$
\begin{aligned}
L_{\mathrm{EM}}^{\prime} & =-\frac{1}{4} F_{\mu \nu}^{\prime} F^{\prime \mu \nu}-J^{\prime \mu} A_{\mu}^{\prime} \\
& =-\frac{1}{4} e^{2(w-1) \rho} F_{\mu \nu} F^{\mu \nu}-e^{\left(w+w_{J}\right) \rho} J^{\mu} A_{\mu},
\end{aligned}
$$

where $w$ and $w_{J}$ are the Weyl weights of $A_{\mu}$ and $J_{\mu}$, respectively. Thus we see that the Lagrangian satisfies (9), provided that $w\left(A_{\mu}\right)=-1$ and $w\left(J_{\mu}\right)=-3$. It is worth noting that the Dirac current $J^{\mu} \equiv \bar{\psi} \gamma^{\mu} \psi$ satisfies this requirement.

\section{B. Local Weyl invariance}

In order to gauge the Weyl transformation (6) and (7), one makes each of the six Lorentz rotation parameters in $\Lambda$, the four translation parameters $a^{\mu}$, and the dilation parameter $\rho$ into eleven independent arbitrary functions of $x$. This leads to a complete decoupling of the translation parts of the transformation from the rotational and dilation parts. Thus, it is customary to view $x^{\prime}$ as arising from a general coordinate transformation (GCT) (resulting in a consequent change in the coordinate basis vectors $\boldsymbol{e}_{\mu}(x)$ at each point), which does not refer at all to the local Lorentz rotation $\Lambda(x)$ of the orthonormal tetrad $\hat{\boldsymbol{e}}_{a}(x)$ or local (physical) dilation $\rho(x)$, at each point. The local Lorentz rotation is still an element of $\mathrm{SO}(3,1)$ (or more precisely $\mathrm{SL}(2, C)$ ) for each $x$, and the basic transformation law for $\varphi$ involves a local shuffling and scaling of components given by

$$
\varphi^{\prime}\left(x^{\prime}\right)=e^{w \rho(x)} S(\Lambda(x)) \varphi(x) .
$$

Since the GCT and the local Lorentz rotation are completely decoupled, it is convenient (and common practice) to use Greek indices on spacetime coordinates $x^{\mu}$ and components of the GCT, but use Latin indices for local Lorentz rotations $\Lambda_{b}^{a}(x)$. This means that any spinor, vector or tensor character that $\varphi$ has in the special-relativistic theory now appears in its local Lorentz transformation law, so that under a GCT each component of $\varphi$ (carrying only Latin indices) behaves as a scalar field. Consequently, the partial derivative, $\partial_{\mu} \varphi$, is covariant under GCT. We note that lowering and raising of Latin indices are performed using the Minkowski metric $\eta_{a b}=\eta^{a b}=\operatorname{diag}(1,-1,-1,-1)$, whereas Greek indices are raised and lowered using the (flat-space) metric $\gamma_{\mu \nu}=\boldsymbol{e}_{\mu} \cdot \boldsymbol{e}_{\nu}$ and its inverse $\gamma^{\mu \nu}$ corresponding to the coordinate system $x^{\mu}$ used to label the Minkowski spacetime. In particular, the duals of the orthonormal tetrad vectors and the coordinate basis vectors are given by $\hat{\boldsymbol{e}}^{a}=\eta^{a b} \hat{\boldsymbol{e}}_{b}$ and $\boldsymbol{e}^{\mu}=\gamma^{\mu v} \boldsymbol{e}_{\nu}$, respectively. Moreover, the decoupling of the GCT from the local Lorentz rotation and local dilation transformations means that the metric $\gamma_{\mu \nu}$ of the Minkowski spacetime obeys the transformation law

$$
\gamma_{\mu \nu}^{\prime}\left(x^{\prime}\right)=\frac{\partial x^{\lambda}}{\partial x^{\prime \mu}} \frac{\partial x^{\sigma}}{\partial x^{\nu}} e^{2 \rho(x)} \gamma_{\lambda \sigma}(x) .
$$

From (17), we see that the transformation law for the derivatives of $\varphi$ under the gauged Weyl transformation is given by

$$
\partial_{\mu}^{\prime} \varphi^{\prime}\left(x^{\prime}\right)=\frac{\partial x^{\nu}}{\partial x^{\prime \mu}} e^{w \rho(x)}\left[\mathrm{S}(\Lambda(x)) \partial_{\nu} \varphi(x)+\partial_{\nu} \mathrm{S}(\Lambda(x)) \varphi(x)+w \partial_{\nu} \rho \mathrm{S}(\Lambda(x)) \varphi(x)\right] .
$$

In order to take advantage of the property (9), we need to construct a covariant derivative that transforms like (8) under local Weyl transformations. This is achieved in two steps. First, one defines the $(\Lambda, \rho)$-covariant derivative of the matter field as ${ }^{65}$

$$
\begin{aligned}
D_{\mu}^{*} \varphi(x) & \equiv\left[\partial_{\mu}+\frac{1}{2} A^{a b}{ }_{\mu}(x) \Sigma_{a b}+w B_{\mu}(x)\right] \varphi(x) \\
& =\left[D_{\mu}+w B_{\mu}(x)\right] \varphi(x),
\end{aligned}
$$


where $D_{\mu} \varphi(x)$ is the $\Lambda$-covariant derivative introduced in PGT; the asterisk on the derivative operator on the left-hand side is intended simply to distinguish it from $D_{\mu} \varphi(x)$ and should not be confused with the operation of complex conjugation. Most importantly, $A^{a b}{ }_{\mu}(x)$ is the gauge-field corresponding to the local Lorentz rotations and $B_{\mu(x)}$ is the gauge-field corresponding to local dilations. The matrices $\Sigma_{a b}=-\Sigma_{a b}$ are the generators of the $\operatorname{SL}(2, C)$ representation $\mathrm{S}(\Lambda)$ to which $\varphi(x)$ belongs, obeying the usual commutation rules

$$
\left[\Sigma_{a b}, \Sigma_{c d}\right]=\eta_{a d} \Sigma_{b c}-\eta_{b d} \Sigma_{a c}+\eta_{b c} \Sigma_{a d}-\eta_{a c} \Sigma_{b d} .
$$

Given the antisymmetry of the generator matrices $\Sigma_{a b}$, without loss of generality we may take the $A$ gauge field to share this antisymmetry property, such that $A^{a b}{ }_{\mu}=-A^{b a}{ }_{\mu}$. The aim of introducing the $A$ and $B$ gauge fields is to compensate for the second and third terms in square brackets in (19), so that the $(\Lambda, \rho)$-covariant derivative transforms under a combined GCT, local Lorentz rotation, and local dilation as

$$
D_{\mu}^{* \prime} \varphi^{\prime}\left(x^{\prime}\right)=\frac{\partial x^{\nu}}{\partial x^{\prime \mu}} e^{w \rho(x)} S(\Lambda(x)) D_{v}^{*} \varphi(x) .
$$

This is easily achieved by demanding that the rotational gauge field $A^{a b}{ }_{\mu}$ transforms as

$$
A^{\prime a b}{ }_{\mu}\left(x^{\prime}\right)=\frac{\partial x^{v}}{\partial x^{\prime \mu}}\left[\Lambda_{c}^{a}(x) \Lambda_{d}^{b}(x) A^{c d}{ }_{\nu}(x)-\Lambda^{b c}(x) \partial_{v} \Lambda_{c}^{a}(x)\right],
$$

and the dilation gauge field $B_{\mu}$ transforms as

$$
B_{\mu}^{\prime}\left(x^{\prime}\right)=\frac{\partial x^{\nu}}{\partial x^{\prime \mu}}\left[B_{v}(x)-\partial_{v} \rho(x)\right]
$$

Thus, $A^{a b}{ }_{\mu}$ and $B_{\mu}$ are covariant vectors under GCT, but transform inhomogeneously under local Lorentz transformations and local dilations, respectively, which is typical of gauge fields. We also note that $A^{a b}{ }_{\mu}$ is invariant under local dilations (i.e., has Weyl weight $w=0$ ).

It is convenient sometimes to write the $(\Lambda, \rho)$-covariant derivative $(20)$ as

$$
D_{\mu}^{*} \varphi(x)=\left[\partial_{\mu}^{*}+\frac{1}{2} A^{a b}{ }_{\mu}(x) \Sigma_{a b}\right] \varphi(x),
$$

where we have defined the derivative operator

$$
\partial_{\mu}^{*} \equiv \partial_{\mu}+w B_{\mu}(x)
$$

It is straightforward to show that $\partial_{\mu}^{*} \varphi(x)$ itself also transforms covariantly with weight $w$ (i.e., the same weight as the field $\varphi$ ) under local dilations (but does not transform covariantly under local Poincaré transformations).

In the second step, we define a generalised covariant derivative, linearly related to $D_{\mu}^{*} \varphi$, by introducing a new, "translational" gauge field $h_{a}{ }^{\mu}(x)$ that transforms via

$$
h_{a}^{\prime \mu}\left(x^{\prime}\right)=\frac{\partial x^{\prime \mu}}{\partial x^{\nu}} e^{-\rho(x)} \Lambda_{a}{ }^{b}(x) h_{b}{ }^{\nu}(x) .
$$

Thus, the field $h_{a}{ }^{\mu}$ is a contravariant GCT vector, a local Lorentz four-vector, and has Weyl weight $w=-1$ under local dilations. The inverse matrix is usually denoted by $b^{a}{ }_{\mu}(x)$ (which has Weyl weight $w=1$ ) and satisfies

$$
h_{a}{ }^{\mu} b^{a}{ }_{\nu}=\delta_{v}^{\mu} \quad \text { and } \quad h_{a}{ }^{\mu} b^{c}{ }_{\mu}=\delta_{a}^{c} .
$$

The generalised covariant derivative is then defined as

$$
\begin{aligned}
\mathcal{D}_{a}^{*} \varphi(x) & \equiv h_{a}{ }^{\mu}(x) D_{\mu}^{*} \varphi(x) \\
& =h_{a}{ }^{\mu}(x)\left[\partial_{\mu}+\frac{1}{2} A^{c d}{ }_{\mu}(x) \Sigma_{c d}+w B_{\mu}(x)\right] \varphi(x),
\end{aligned}
$$

which consequently transforms under local Weyl transformations in the same way as $\partial \varphi$ does under global Weyl transformations, namely, ${ }^{66}$

$$
\mathcal{D}_{a}^{* \prime} \varphi^{\prime}\left(x^{\prime}\right)=e^{(w-1) \rho(x)} \Lambda_{a}{ }^{b}(x) \mathrm{S}(\Lambda(x)) \mathcal{D}_{b}^{*} \varphi(x) .
$$


Having achieved our goal of defining a general covariant derivative with the transformation law (30), we can take advantage of the property (9) of the special-relativistic matter Lagrangian, and we see that under the local Weyl group one has

$$
L_{\mathrm{M}}\left(\varphi^{\prime}\left(x^{\prime}\right) ; \mathcal{D}_{a}^{* \prime} \varphi^{\prime}\left(x^{\prime}\right)\right)=e^{-4 \rho(x)} L_{\mathrm{M}}\left(\varphi(x) ; \mathcal{D}_{a}^{*} \varphi(x)\right) .
$$

Thus the new minimally coupled matter Lagrangian is a GCT and local Lorentz scalar and has a Weyl weight $w=-4$. However, since $x \rightarrow x^{\prime}$ is a GCT, we need a scalar density, rather than a scalar, as the integrand in the matter action. Moreover, the integrand must have an overall Weyl weight of zero. Thus we must multiply $L_{\mathrm{M}}\left(\varphi ; \mathcal{D}^{*} \varphi\right)$ by a factor which transforms by the appropriate Jacobian determinant of the GCT and has a Weyl weight $w=4$. From the transformation properties of the $A-, B-$, and $h$-fields, we see that the only suitable factor that does not involve derivatives is $h^{-1}$. Thus, our final minimally coupled matter action is

$$
S_{\mathrm{M}}=\int h^{-1} L_{\mathrm{M}}\left(\varphi, \mathcal{D}_{a}^{*} \varphi\right) d^{4} x
$$

which is invariant under local Weyl transformations. It will be convenient to denote the integrand of the action by the Lagrangian density $\mathcal{L}_{\mathrm{M}} \equiv h^{-1} L_{\mathrm{M}}\left(\varphi, \mathcal{D}_{a}^{*} \varphi\right)$. We note that the gravitational gauge fields $h, A$, and $B$ contain a total of 44 independent variables.

It should be mentioned, however, that the locally Weyl-invariant matter action (32) is not guaranteed to inherit invariance properties possessed by the original action (5) under other types of transformation, so (32) may need to be modified to restore any further required invariances. An important example that illustrates this phenomenon is provided by the electromagnetic field and is discussed in Section II K. For the purposes of our general discussion, however, we will assume that the action has the form (32).

As mentioned previously, one may also introduce an additional "compensator" scalar field $\phi$ (or fields) into the matter action. The introduction of an additional scalar field opens up possibilities for the inclusion of further terms in the matter action that non-minimally (conformally) couple $\phi$ to the WGT gravitational gauge field strengths (see Section II D). In particular, terms proportional to $\phi^{2} \mathcal{R}$ or $\phi^{2} L_{\mathcal{T}^{* 2}}$ are Weyl-covariant with weight $w=-4$ and so may be added to $L_{\mathrm{M}}$ (where $L_{\mathcal{T}^{* 2}}$ has the form (3), but with the PGT translational gauge field strength replaced by the corresponding quantity in WGT).

\section{Minkowski spacetime interpretation of WGT}

At this point, it is worth making a few observations that are not usually presented in the literature. First, we have introduced the $h, A$, and $B$ fields as gravitational gauge fields in Minkowski spacetime, in keeping with the interpretation of the gauge field theories describing the other fundamental interactions. We are therefore still at liberty to perform our calculations in a global inertial Cartesian coordinate system $x^{\mu}$. In making this choice, one may extend the $(\Lambda, \rho)$-covariant derivative $D_{\mu}^{*}$ to act on any quantity that transforms linearly under local Lorentz transformations, even if it also carries Greek indices, by simply ignoring its GCT transformation properties, since the explicit form for the generators $\Sigma_{a b}$ appearing in $D_{\mu}^{*} \varphi$ depends only on the Lorentz transformation properties of $\varphi$. Thus, for instance, considering the $h$-field itself, one has

$$
D_{\mu}^{*} h_{a}^{v}=\partial_{\mu}^{*} h_{a}^{v}+\frac{1}{2} A^{c d}{ }_{\mu}\left(\Sigma_{c d}^{1}\right)_{a}^{b} h_{b}{ }^{v}=\partial_{\mu}^{*} h_{a}{ }^{v}-A^{b}{ }_{a \mu} h_{b}{ }^{v},
$$

where $\left(\Sigma_{c d}^{1}\right)_{b}^{a}=\left(\delta_{c}^{a} \eta_{d b}-\delta_{d}^{a} \eta_{c b}\right)$ are the generators of the vector representation of the Lorentz group. Similarly, for the inverse $h$-field,

$$
D_{\mu}^{*} b^{a}{ }_{\nu}=\partial_{\mu}^{*} b^{a}{ }_{\nu}+A^{a}{ }_{b \mu} b^{b}{ }_{\nu} .
$$

It may prove useful occasionally, however, to work in a different coordinate system in Minkowski spacetime, such as spherical polar coordinates. In this case, we are obliged to generalise the $(\Lambda, \rho)$-covariant derivative so that it is capable of being applied to fields carrying Greek indices, which have definite tensor behaviour under GCT, in addition to $\operatorname{SL}(2, C)$ behaviour. In this case, the 
$(\Lambda, \rho)$-covariant derivative in $(25)$ becomes

$$
D_{\mu}^{*} \equiv \partial_{\mu}^{*}+{ }^{0} \Gamma_{\rho \mu}^{\sigma} \mathrm{X}_{\sigma}{ }_{\sigma}+\frac{1}{2} A^{a b}{ }_{\mu} \Sigma_{a b}={ }^{0} \nabla_{\mu}^{*}+\frac{1}{2} A^{a b}{ }_{\mu} \Sigma_{a b},
$$

where ${ }^{0} \nabla_{\mu}^{*} \equiv{ }^{0} \nabla_{\mu}+w B_{\mu},{ }^{0} \Gamma_{\mu \nu}^{\lambda} \equiv \frac{1}{2} \gamma^{\lambda \rho}\left(\partial_{\mu} \gamma_{\nu \rho}+\partial_{\nu} \gamma_{\mu \rho}-\partial_{\rho} \gamma_{\mu \nu}\right)$ is the metric connection corresponding to the (flat-space) metric $\gamma_{\mu \nu}$ defined by the coordinate system, and $\mathrm{X}^{\rho}{ }_{\sigma}$ are the GL $(4, R)$ generator matrices appropriate to the GCT tensor character of the field to which $D_{\mu}^{*}$ is applied, and $w$ is its Weyl weight. Once again taking the $h$-field as an example, one thus has

$$
D_{\mu}^{*} h_{a}{ }^{\nu}=\partial_{\mu}^{*} h_{a}{ }^{v}+{ }^{0} \Gamma^{\nu}{ }_{\rho \mu} h_{a}{ }^{\rho}-A^{b}{ }_{a \mu} h_{b}{ }^{\nu} .
$$

It should be remembered, however, that in Minkowski spacetime, the connection coefficients ${ }^{0} \Gamma^{\sigma}{ }_{\rho \mu}$ are not dynamical entities, but are fixed by our choice of coordinate system. Moreover, the curvature and torsion tensors derived from the connection both vanish in Minkowski spacetime, meaning that the derivatives ${ }^{0} \nabla_{\mu}$ and ${ }^{0} \nabla_{\nu}$ always commute, i.e.,

$$
\left[{ }^{0} \nabla_{\mu}, \nabla_{\nu}\right] \varphi=0
$$

irrespective of the GCT tensor character of $\varphi$. Unless otherwise stated, however, from now on we will adopt a global Cartesian inertial coordinate system $x^{\mu}$ in our Minkowski spacetime, which greatly simplifies calculations.

Finally, some further comments about the interpretation of the translational gauge field are worthwhile. It is a rank-2 tensor field, which we will denote by $\boldsymbol{h}$, defined on Minkowski spacetime. Thus, formally, it provides a linear mapping from any two vectors in Minkowski spacetime to the real numbers. In the usual manner, the components of $\boldsymbol{h}$ that we have considered hitherto are given by $h_{a}{ }^{\mu} \equiv \boldsymbol{h}\left(\hat{\boldsymbol{e}}_{a}, \boldsymbol{e}^{\mu}\right)$. Similarly, the inverse of $\boldsymbol{h}$, which we denote by $\boldsymbol{b}$, is also a rank-2 tensor field defined on Minkowski spacetime, and the components that we have considered so far are given by $b^{a}{ }_{\mu} \equiv \boldsymbol{b}\left(\hat{\boldsymbol{e}}^{a}, \boldsymbol{e}_{\mu}\right)$. Other components of $\boldsymbol{h}$ and $\boldsymbol{b}$ may be obtained in the usual manner by acting with them on different combinations of the tetrad and coordinate basis vectors and their duals. The two tensors $\boldsymbol{h}$ and $\boldsymbol{b}$ are inverses in the sense that the components $h_{a}{ }^{\mu}$ and $b^{a}{ }_{\mu}$ satisfy the conditions (28).

Given their transformation properties, the components of $\boldsymbol{h}$ and $\boldsymbol{b}$ are able to convert between $\mathrm{GCT}$ tensor behaviour and the corresponding $\operatorname{SL}(2, C)$ behaviour, i.e., they can be used to replace Greek indices with Latin ones and vice versa (although $\operatorname{SL}(2, C)$ spinors cannot be converted into any GCT tensorial objects). As an example, let us consider the simplest case of a vector field $\boldsymbol{J}$ defined on the Minkowski spacetime, which may be written in terms of its components in the coordinate basis or its dual as $\boldsymbol{J}=J^{\mu} \boldsymbol{e}_{\mu}=J_{\mu} \boldsymbol{e}^{\mu}$. Replacing the Greek index with a Latin one using the components of $\boldsymbol{h}$, we may obtain the quantities $\mathcal{J}_{a} \equiv h_{a}{ }^{\mu} J_{\mu}=h_{a \mu} J^{\mu}$ or $\mathcal{J}^{a} \equiv h^{a}{ }_{\mu} J^{\mu}=h^{a \mu} J_{\mu}$. Adopting the viewpoint that $\boldsymbol{h}$ is a tensor (gauge) field in Minkowski spacetime, and thus attaching to it no geometric significance a priori, one must regard these as the components in the local tetrad basis of a new vector field $\mathcal{J}=\boldsymbol{h}(\cdot, \boldsymbol{J}) \cdot{ }^{67}$ It is also worth noting that the components of $\boldsymbol{h}$ may also be used to convert Latin indices into Greek ones. For example, consider the vector $\boldsymbol{h}(\mathcal{J}, \cdot)$, which has contravariant components in the coordinate basis given by $h_{a}{ }^{\mu} \mathcal{J}^{a}$; these are the components of another new vector and are, in general, not equal to the original $J_{\mu}$.

Similar conclusions follow for the inverse tensor $\boldsymbol{b}$. For example, if we place $\mathcal{J}$ into the first "slot" of $\boldsymbol{b}$, we recover the original vector $\boldsymbol{J}=\boldsymbol{b}(\mathcal{J}, \cdot)$, since its covariant components in the coordinate basis are $b^{a}{ }_{\mu} \mathcal{J}_{a}=J_{\mu}$, where in the last equality we have used the reciprocity relations (28). These components can also be written as $J_{\mu}=b_{a \mu} \mathcal{J}^{a}$, and their contravariant counterparts as $J^{\mu}=b_{a}{ }^{\mu} \mathcal{J}^{a}=b^{a \mu} \mathcal{J}_{a}$. In all these cases, the components of $\boldsymbol{b}$ have been used to convert a Latin index into a Greek one. One may also perform the reverse operation: consider, for example, the vector $\boldsymbol{b}(\cdot, \boldsymbol{J})$; this has contravariant components in the tetrad basis given by $b^{a}{ }_{\mu} J^{\mu}$, but these are not, in general, equal to $\mathcal{J}^{a}$.

The index conversion properties of the components of $\boldsymbol{h}$ and $\boldsymbol{b}$ trivially extend to objects possessing multiple indices: for example, if $\boldsymbol{T}$ is a rank- 2 tensor field, then one obtains the new rank-2 tensor field $\mathcal{T}$ with covariant components (say) in the tetrad basis given by $\mathcal{T}_{a b}=h_{a}{ }^{\mu} h_{b}{ }^{\nu} T_{\mu \nu}$. Using the reciprocity relations (28), one may therefore also write, for example, $T_{\mu \nu}=b^{a}{ }_{\mu} b^{b}{ }_{\nu} \mathcal{T}_{a b}$. One may also, of course, convert just some subset of the indices on a higher-rank object, and each case 
corresponds to the components of a different object, referred to the corresponding combination of tetrad and coordinate basis vectors. ${ }^{68}$

The interpretation presented above contrasts sharply with the (more commonly adopted) geometric interpretation of WGT (which we describe in detail in Section II O), in which $h_{a}{ }^{\mu}$ is identified as a vierbein that directly relates the coordinate basis vectors $\boldsymbol{e}_{\mu}$ and local tetrad frame vectors $\hat{\boldsymbol{e}}_{a}$; in this case, the components $h_{a}{ }^{\mu} J_{\mu}$, for example, are interpreted as the components $J_{a}$ of the original vector field $\boldsymbol{J}$ in the local Lorentz basis. Moreover, in the Minkowski spacetime gauge field interpretation, the RHS of (36) need not vanish, which should be contrasted with the usual "tetrad postulate" that applies in the geometric interpretation of WGT.

\section{Gauge field strengths in WGT}

The gauge field strengths in WGT are defined in the usual way in terms of the commutator of the covariant derivatives. Considering first the $(\Lambda, \rho)$-covariant derivative, one finds

$$
\left[D_{\mu}^{*}, D_{v}^{*}\right] \varphi=\frac{1}{2} R^{a b}{ }_{\mu \nu} \Sigma_{a b} \varphi+w H_{\mu \nu} \varphi,
$$

where we have defined the "rotational" and "dilation" field strength tensors of the gauge fields $A^{a b}{ }_{\mu}$ and $B_{\mu}$, respectively, as

$$
\begin{aligned}
R^{a b}{ }_{\mu \nu} & \equiv \partial_{\mu} A^{a b}{ }_{\nu}-\partial_{\nu} A^{a b}{ }_{\mu}+A^{a}{ }_{c \mu} A^{c b}{ }_{\nu}-A^{a}{ }_{c \nu} A^{c b}{ }_{\mu}, \\
H_{\mu \nu} & \equiv \partial_{\mu} B_{\nu}-\partial_{\nu} B_{\mu} .
\end{aligned}
$$

Both $R^{a b}{ }_{\mu \nu}$ and $H_{\mu \nu}$ transform covariantly under GCT and local Lorentz rotations in accordance with their respective index structures and are invariant under local dilations (i.e., each has Weyl weight $w=0$ ).

Of greater relevance, however, is the commutator of two generalised covariant derivatives. Since $\mathcal{D}_{a}^{*} \varphi=h_{a}{ }^{\mu} D_{\mu}^{*} \varphi$, this commutator differs from (38) by an additional term containing the derivatives of $h_{a}{ }^{\mu}$ and reads

$$
\left[\mathcal{D}_{c}^{*}, \mathcal{D}_{d}^{*}\right] \varphi=\frac{1}{2} \mathcal{R}^{a b}{ }_{c d} \Sigma_{a b} \varphi+w \mathcal{H}_{c d} \varphi-\mathcal{T}^{* a}{ }_{c d} \mathcal{D}_{a}^{*} \varphi,
$$

where $\mathcal{R}^{a b}{ }_{c d} \equiv h_{a}{ }^{\mu} h_{b}{ }^{\nu} R^{a b}{ }_{\mu \nu}$ and $\mathcal{H}_{c d}=h_{c}{ }^{\mu} h_{d}{ }^{\nu} H_{\mu \nu}$, and the "translational" field strength of the gauge field $h_{a}{ }^{\mu}$ is given by

$$
\mathcal{T}^{* a}{ }_{b c} \equiv h_{b}{ }^{\mu} h_{c}{ }^{\nu} T^{* a}{ }_{\mu \nu} \equiv h_{b}{ }^{\mu} h_{c}{ }^{\nu}\left(D_{\mu}^{*} b^{a}{ }_{\nu}-D_{\nu}^{*} b^{a}{ }_{\mu}\right),
$$

which transforms covariantly under local Lorentz transformations in accordance with its index structure. It is worth noting that (42) can also be written as

$$
\mathcal{T}^{* a}{ }_{b c}=-b^{a}{ }_{\mu}\left(\mathcal{D}_{b}^{*} h_{c}{ }^{\mu}-\mathcal{D}_{c}^{*} h_{b}{ }^{\mu}\right) .
$$

One may easily show that the translational field strength in WGT is related to the corresponding field strength $\mathcal{T}^{a}{ }_{b c}$ in PGT (see Appendix B) by

$$
\mathcal{T}^{* a}{ }_{b c}=\mathcal{T}^{a}{ }_{b c}+\delta_{c}^{a} \mathcal{B}_{b}-\delta_{b}^{a} \mathcal{B}_{c},
$$

where $\mathcal{B}_{a}=h_{a}{ }^{\mu} B_{\mu}$. It is also convenient to define $\mathcal{T}_{b}{ }^{*} \equiv \mathcal{T}^{* a}{ }_{b a}=\mathcal{T}_{b}+3 \mathcal{B}_{b}$, where $\mathcal{T}_{b}$ is the corresponding PGT quantity.

It is easy to show that $\mathcal{R}^{a b}{ }_{c d}, \mathcal{H}_{c d}$, and $\mathcal{T}^{* a}{ }_{c d}$ transform covariantly under local dilations, with weights $w\left(\mathcal{R}^{a b}{ }_{c d}\right)=w\left(\mathcal{H}_{c d}\right)=-2$ and $w\left(\mathcal{T}^{* a}{ }_{c d}\right)=-1$, respectively. We further note that the functional dependencies of the three field strengths tensors on the gauge fields and their first derivatives are $\mathcal{R}^{a b}{ }_{c d}(h, A, \partial A), \mathcal{T}^{* a}{ }_{b c}(h, \partial h, A, B)$, and $\mathcal{H}_{a b}(h, \partial B)$. When WGT is re-interpreted geometrically (see Section II O), the field strengths $\mathcal{R}^{a b}{ }_{c d}, \mathcal{H}_{a b}$, and $\mathcal{T}^{* a}{ }_{b c}$ are related to the curvature and torsion tensors of a Weyl-Cartan $Y_{4}$ spacetime, but here are to be regarded simply as local tensors defined in Minkowski spacetime. 


\section{E. Alternative form of covariant derivative in WGT}

An immediate use of the expression (43) is that it can be rearranged to give an important expression for the $A$ gauge field. Defining the quantities

$$
c^{* a}{ }_{b c} \equiv h_{b}{ }^{\mu} h_{c}{ }^{\nu}\left(\partial_{\mu}^{*} b^{a}{ }_{\nu}-\partial_{\nu}^{*} b^{a}{ }_{\mu}\right),
$$

the relevant relationship is easily found to be $A_{a b \mu}=b^{c}{ }_{\mu} \mathcal{A}_{a b c}$, where

$$
\mathcal{A}_{a b c}=\frac{1}{2}\left(c_{a b c}^{*}+c_{b c a}^{*}-c_{c a b}^{*}\right)-\frac{1}{2}\left(\mathcal{T}_{a b c}^{*}+\mathcal{T}_{b c a}^{*}-\mathcal{T}_{c a b}^{*}\right) .
$$

It is important to note that this is an identity that is valid independently of any equations of motion satisfied by the gauge fields $h, A$, and $B$. In the special case where the equations of motion result in $\mathcal{T}_{a b c}^{*}$ being independent of $\mathcal{A}_{a b c}$, (46) gives an explicit expression for the $A$-field in terms of the $h$-field, its first derivatives, and the $B$-field. In such a case, the $A$-field is no longer an independent gauge field and the resulting theory can, if desired, be written entirely in terms of the other gauge fields, i.e., one moves from a "first-order" to a "second-order" formalism.

It will prove convenient for our later discussion to rewrite the expression (46) as

$$
\mathcal{A}_{a b c}={ }^{0} \mathcal{A}_{a b c}^{*}(h, \partial h, B)+\mathcal{K}_{a b c}^{*}(h, \partial h, A, B),
$$

in which we have defined the quantities ${ }^{0} \mathcal{A}_{a b c}^{*} \equiv \frac{1}{2}\left(c_{a b c}^{*}+c_{b c a}^{*}-c_{c a b}^{*}\right)$ that, as indicated, are fully determined by the $h$-field, its first derivatives, and the $B$-field. We have also defined $\mathcal{K}_{a b c}^{*} \equiv$ $-\frac{1}{2}\left(\mathcal{T}_{a b c}^{*}+\mathcal{T}_{b c a}^{*}-\mathcal{T}_{c a b}^{*}\right)$, which when re-interpreted geometrically is analogous to the contortion tensor of a Weyl-Cartan spacetime $Y_{4}$. It is worth noting that both ${ }^{0} \mathcal{A}_{a b c}^{*}$ and $\mathcal{K}_{a b c}^{*}$ are antisymmetric in their first two indices. ${ }^{69}$

Under a local Weyl transformation, the quantities ${ }^{0} \mathcal{A}_{a b c}^{*}$ transform in the same way as $\mathcal{A}_{a b c}^{*}$, whereas $\mathcal{K}_{a b c}^{*}$ transform as the components of a local tensor with weight $w=-1$. Thus, the quantities ${ }^{0} \mathcal{A}_{a b c}^{*}$ can serve equally well as $\mathcal{A}_{a b c}^{*}$ in the construction of a covariant derivative. We may therefore construct the "reduced" $(\Lambda, \rho)$-covariant derivative

$$
{ }^{0} D_{\mu}^{*} \varphi \equiv\left(\partial_{\mu}^{*}+\frac{1}{2}^{0} A^{* a b}{ }_{\mu} \Sigma_{a b}\right) \varphi
$$

which transforms according to (22) under a local Weyl transformation, in the same way as $D_{\mu}^{*} \varphi$, but depends only on the $h$ field, its first derivatives, and the $B$-field. Similarly, one can define the corresponding generalised covariant derivative ${ }^{0} \mathcal{D}_{a}^{*} \equiv h_{a}{ }^{\mu 0} D_{\mu}^{*}$, which consequently transforms as in (30), as required. The "full" generalised covariant derivative is given in terms of the "reduced" one by the alternative form

$$
\mathcal{D}_{a}^{*} \varphi=\left({ }^{0} \mathcal{D}_{a}^{*}+\frac{1}{2} \mathcal{K}^{* b c}{ }_{a} \Sigma_{b c}\right) \varphi,
$$

which makes clear that if one (covariantly) sets the WGT torsion (and hence contortion) to zero, then $\mathcal{D}_{a}^{*}$ reduces to ${ }^{0} \mathcal{D}_{a}^{*}$.

\section{F. Bianchi identities in WGT}

It will also be convenient for our later development to obtain the Bianchi identities satisfied by the gravitational gauge field strengths $\mathcal{R}^{a b}{ }_{c d}, \mathcal{T}^{* a}{ }_{b c}$, and $\mathcal{H}_{a b}$ in WGT. These may be straightforwardly derived from the Jacobi identity applied to the generalised covariant derivative, namely,

$$
\left[\mathcal{D}_{a}^{*},\left[\mathcal{D}_{b}^{*}, \mathcal{D}_{c}^{*}\right]\right] \varphi+\left[\mathcal{D}_{c}^{*},\left[\mathcal{D}_{a}^{*}, \mathcal{D}_{b}^{*}\right]\right] \varphi+\left[\mathcal{D}_{b}^{*},\left[\mathcal{D}_{c}^{*}, \mathcal{D}_{a}^{*}\right]\right] \varphi=0 .
$$

Inserting the form (29) for the generalised covariant derivative into the above Jacobi identity, one quickly finds the three Bianchi identities

$$
\begin{aligned}
\mathcal{D}_{[a}^{*} \mathcal{R}_{b c]}^{d e}-\mathcal{T}^{* f}{ }_{[a b} \mathcal{R}_{c] f}^{d e} & =0, \\
\mathcal{D}_{[a}^{*} \mathcal{T}^{* d}{ }_{b c]}-\mathcal{T}^{* e}{ }_{[a b} \mathcal{T}^{* d}{ }_{c] e}-\mathcal{R}_{[a b c]}^{d}+\mathcal{H}_{[a b} \delta_{c]}^{d} & =0, \\
\mathcal{D}_{[a}^{*} \mathcal{H}_{b c]}-\mathcal{T}^{* e}{ }_{[a b} \mathcal{H}_{c] e} & =0 .
\end{aligned}
$$

It is worth noting that for the special case in which the "torsion" is totally antisymmetric, such that $\mathcal{T}_{a b c}^{*}=\epsilon_{a b c d} t^{d}$ for some vector $t^{d}$, then the second term on the LHS of (52) vanishes. 
By contracting over the indices $a$ and $d$ in the "R-identity" (51), once obtains the oncecontracted Bianchi identity

$$
\mathcal{D}_{a}^{*} \mathcal{R}^{a e}{ }_{b c}-2 \mathcal{D}_{[b}^{*} \mathcal{R}_{c]}^{e}-2 \mathcal{T}^{* f}{ }_{a[b} \mathcal{R}^{a e}{ }_{c] f}-\mathcal{T}^{* f}{ }_{b c} \mathcal{R}_{f}^{e}=0 .
$$

Contracting again over $b$ and $e$, one then finds the twice-contracted Bianchi identity

$$
\mathcal{D}_{a}^{*}\left(\mathcal{R}^{a}{ }_{c}-\frac{1}{2} \delta_{c}^{a} \mathcal{R}\right)+\mathcal{T}^{* f}{ }_{b c} \mathcal{R}^{b}{ }_{f}+\frac{1}{2} \mathcal{T}^{* f}{ }_{a b} \mathcal{R}^{a b}{ }_{c f}=0 .
$$

Turning to the "T -identity" (52) and contracting over $a$ and $d$, one obtains the further oncecontracted Bianchi identity

$$
\mathcal{D}_{a}^{*} \mathcal{T}^{* a}{ }_{b c}+2 \mathcal{D}_{[b}^{*} \mathcal{T}_{c]}^{*}+\mathcal{T}^{* e}{ }_{b c} \mathcal{T}_{e}^{*}+2 \mathcal{R}_{[b c]}+2 \mathcal{H}_{b c}=0
$$

One should note that (56) simplifies considerably if the torsion trace $\mathcal{T}_{a}^{*}$ vanishes, which clearly includes the special case in which the torsion is totally antisymmetric. It is clear that the " $\mathcal{H}$-identity" (53) has no non-trivial contractions.

\section{G. Free gravitational action in WGT}

In addition to the locally Weyl-invariant matter action (32), one must also include in the total action some further terms, which describe the dynamics of the free gravitational gauge fields. From $\mathcal{R}_{a b c d}, \mathcal{T}_{a b c}^{*}$, and $\mathcal{H}_{a b}$, one can construct a free gravitational action of the general form

$$
S_{\mathrm{G}}=\int h^{-1} L_{\mathrm{G}}\left(\mathcal{R}_{a b c d}, \mathcal{T}_{a b c}^{*}, \mathcal{H}_{a b}\right) d^{4} x,
$$

where the requirement of local scale invariance imposes the constraint that $L_{\mathrm{G}}$ must be a relative scalar with Weyl weight $w\left(L_{\mathrm{G}}\right)=-4$, so that the Lagrangian density $\mathcal{L}_{\mathrm{G}} \equiv h^{-1} L_{\mathrm{G}}$ has weight zero (i.e., scale invariant). It is trivial to show that $\mathcal{R}_{a b c d}, \mathcal{H}_{a b}$, and $\mathcal{T}_{a b c}^{*}$ transform covariantly under local dilations with Weyl weights $w\left(\mathcal{R}_{a b c d}\right)=w\left(\mathcal{H}_{a b}\right)=-2$ and $w\left(\mathcal{T}_{a b c}^{*}\right)=-1$, respectively. ${ }^{70}$ Hence $L_{\mathrm{G}}$ can be quadratic in $\mathcal{R}_{a b c d}$ and $\mathcal{H}_{a b}$, but terms linear in $\mathcal{R} \equiv \mathcal{R}^{a b}{ }_{a b}$ or quadratic in $\mathcal{T}_{a b c}^{*}$ are not allowed. Similarly, higher-order terms in $\mathcal{R}_{a b c d}$ and $\mathcal{H}_{a b}$ are forbidden; in principle one could include quartic terms in $\mathcal{T}_{a b c}^{*}$, but we will not consider them here. Thus, in WGT, the general form of $L_{\mathrm{G}}$, possessing terms no higher than quadratic order in the field strengths, is of the form

$$
L_{\mathrm{G}}=L_{\mathcal{R}^{2}}+L_{\mathcal{H}^{2}}
$$

where the expressions for $L_{\mathcal{R}^{2}}$ and $L_{\mathcal{H}^{2}}$ are given in (4). It may be shown, however, that the field strength $\mathcal{R}_{a b c d}$ satisfies a form of Gauss-Bonnet identity, ${ }^{71}$ such that the combination

$$
\mathcal{R}^{2}-4 \mathcal{R}_{a b} \mathcal{R}^{b a}+\mathcal{R}_{a b c d} \mathcal{R}^{c d a b}
$$

contributes a total derivative to the action (in $D \leq 4$ dimensions), and so has no effect on the resulting field equations. Hence one may set any one of $\alpha_{1}, \alpha_{3}$, or $\alpha_{6}$ in (4) to zero, without loss of generality (at least classically), but we will retain all these terms for the moment. As mentioned in Section II B, we note that one may also include in the total Lagrangian terms of the generic form $\phi^{2} \mathcal{R}$ and $\phi^{2} L_{\mathcal{T}^{* 2}}$, where $L_{\mathcal{T}^{* 2}}$ is given in (3), but with $\mathcal{T}_{a b c} \rightarrow \mathcal{T}_{a b c}^{*}$. However, since these terms depend upon the scalar (compensator) field $\phi$, in addition to the gravitational gauge fields, we do not consider them to be part of the free gravitational action, but instead regard them as belonging to the matter Lagrangian $L_{\mathrm{M}}$ (see Section II J).

The precise form of WGT under consideration depends on the form of the free gravitational Lagrangian density $\mathcal{L}_{\mathrm{G}}$. As an illustration, in Appendix $\mathrm{D}$ we give a brief account of a scale-invariant theory of gravity suggested by Dirac (but extended to include torsion), which can accommodate "ordinary" matter via the inclusion of an additional "compensator" scalar field. In this theory, $L_{\mathrm{G}}=L_{\mathcal{H}^{2}}$ and $L_{\mathrm{M}}$ contains a term proportional to $\phi^{2} \mathcal{R}$. 


\section{H. Field equations in WGT}

The total action $S_{\mathrm{T}}$ is simply the sum of the matter and free gravitational actions. In the free gravitational sector, the form of the Lagrangian (58) induces a dependence on $h, A, \partial A$, and $\partial B$ (suppressing indices for brevity). Note, in particular, that the absence of $\mathcal{T}_{a b c}^{*}$ from (58) means that it does not contain derivatives of the $h$-field. In the matter sector, covariant derivatives of the matter field $\varphi$ induce a dependence on $\varphi, \partial \varphi, h, A$, and $B$. We will also consider here the inclusion of an additional "compensator" scalar field $\phi$ in the matter action and further admit the possibility that it may include a kinetic term for the scalar field that contains derivatives of $\phi$. Moreover, if the matter action includes a term proportional to $\phi^{2} \mathcal{R}$, then this brings an additional dependence on $\partial A$. Finally, if one includes terms of the generic form $\phi^{2} L_{\mathcal{T}^{* 2}}$, then it produces an additional dependence on $\partial h$. Consequently, we will take the total Lagrangian density to be

$$
\mathcal{L}_{\mathrm{T}}=\mathcal{L}_{\mathrm{G}}(h, A, \partial A, \partial B)+\mathcal{L}_{\mathrm{M}}(\varphi, \partial \varphi, \phi, \partial \phi, h, \partial h, A, \partial A, B),
$$

where we have indicated the functional dependencies in the most general case.

Since $\mathcal{L}_{\mathrm{T}}$ is at most quadratic in the field strength tensors $\mathcal{R}_{a b c d}(h, A, \partial A), \mathcal{T}_{a b c}^{*}(h, \partial h, A, B)$, and $\mathcal{H}_{a b}(h, \partial B)$, and each of these is linear in the first derivative of the corresponding gauge field, the resulting equations of motion are, in general, linear in the second-order derivatives of the $h, A$, and $B$ gauge fields and contain no higher-derivative terms; such theories therefore do not suffer from Ostrogradsky's instability. It is worth noting, however, that if one substitutes for $\mathcal{A}_{a b c}$ using (47), one obtains equations of motion that contain up to third-order derivatives of the $h$-field, which can lead to difficulties in the interpretation of initial-value problems.

Variation of $S_{\mathrm{T}}$ with respect to the gauge fields $h_{a}{ }^{\mu}, A^{a b}{ }_{\mu}$, and $B_{\mu}$ leads to three coupled gravitational field equations for the gauge fields. In general, these read

$$
\begin{aligned}
t^{a}{ }_{\mu}+\tau^{a}{ }_{\mu} & =0, \\
s_{a b}{ }^{\mu}+\sigma_{a b}{ }^{\mu} & =0, \\
j^{\mu}+\zeta^{\mu} & =0 .
\end{aligned}
$$

In the free gravitational sector, we have defined $t^{a}{ }_{\mu} \equiv \delta \mathcal{L}_{\mathrm{G}} / \delta h_{a}{ }^{\mu}=\partial \mathcal{L}_{\mathrm{G}} / \partial h_{a}{ }^{\mu}, s_{a b}{ }^{\mu} \equiv \delta \mathcal{L}_{\mathrm{G}} / \delta A^{a b}{ }_{\mu}$, and $j^{\mu} \equiv \delta \mathcal{L}_{\mathrm{G}} / \delta B_{\mu}$, respectively. In the matter sector, the energy-momentum $\tau^{a}{ }_{\mu} \equiv \delta \mathcal{L}_{\mathrm{M}} / \delta h_{a}{ }^{\mu}$, spinangular-momentum $\sigma_{a b}{ }^{\mu} \equiv \delta \mathcal{L}_{\mathrm{M}} / \delta A^{a b}{ }_{\mu}$, and dilation current $\zeta^{\mu} \equiv \delta \mathcal{L}_{\mathrm{M}} / \delta B_{\mu}=\partial \mathcal{L}_{\mathrm{M}} / \partial B_{\mu}$ act as sources. ${ }^{72}$ It is easy to show that all the quantities in (61a)-(61c) transform covariantly under local Lorentz rotations and GCTs in accordance with their respective index structures and are also covariant under local dilations with Weyl weights $w=1, w=0$, and $w=0$, respectively. It is, in fact, more convenient to work with quantities carrying only Latin indices and write the general field equations as

$$
\begin{aligned}
t^{a}{ }_{b}+\tau^{a}{ }_{b} & =0, \\
s_{a b}{ }^{c}+\sigma_{a b}{ }^{c} & =0, \\
j^{a}+\zeta^{a} & =0,
\end{aligned}
$$

where $t^{a}{ }_{b} \equiv t^{a}{ }_{\mu} h_{b}{ }^{\mu}, s_{a b}{ }^{c} \equiv s_{a b}{ }^{\mu} b^{c}{ }_{\mu}$, and $j^{a} \equiv j^{\mu} b^{a}{ }_{\mu}$, and similarly for the matter sector. ${ }^{73}$ All the quantities in (62a)-(62c) are clearly invariant under GCT, transform covariantly under local Lorentz rotations in accordance with their respective index structures, and are also covariant under local dilations with Weyl weights $w=0, w=1$, and $w=1$, respectively.

Varying $S_{\mathrm{T}}$ with respect to $\varphi$ and $\phi$ leads to the matter field equations. Considering just the $\varphi$-equation for the moment, since $\mathcal{L}_{\mathrm{G}}$ does not depend on $\varphi$, one has simply

$$
\frac{\delta \mathcal{L}_{\mathrm{M}}}{\delta \varphi} \equiv \frac{\partial \mathcal{L}_{\mathrm{M}}}{\partial \varphi}-\partial_{\mu}\left(\frac{\partial \mathcal{L}_{\mathrm{M}}}{\partial\left(\partial_{\mu} \varphi\right)}\right)=0 .
$$

One may straightforwardly show that this equation can be recast in terms of the $(\Lambda, \rho)$-covariant derivative (20) as

$$
\frac{\delta \mathcal{L}_{\mathrm{M}}}{\delta \varphi} \equiv \frac{\bar{\partial} \mathcal{L}_{\mathrm{M}}}{\partial \varphi}-D_{\mu}^{*}\left(\frac{\partial \mathcal{L}_{\mathrm{M}}}{\partial\left(D_{\mu}^{*} \varphi\right)}\right)=0
$$


where $\bar{\partial} \mathcal{L}_{\mathrm{M}} / \partial \varphi \equiv\left[\partial \mathcal{L}_{\mathrm{M}}\left(\varphi, \mathcal{D}_{\mu}^{*} u, \ldots\right) / \partial \varphi\right]_{u=\varphi}$, so that $\varphi$ and $\mathcal{D}_{\mu}^{*} \varphi$ are treated as independent variables, rather than $\varphi$ and $\partial_{\mu} \varphi$. Even more convenient for our purposes is to rewrite (64) in terms of the generalised covariant derivative (29), which yields

$$
\frac{\bar{\partial} L_{\mathrm{M}}}{\partial \varphi}-\mathcal{D}_{a}^{*}\left(\frac{\partial L_{\mathrm{M}}}{\partial\left(\mathcal{D}_{a}^{*} \varphi\right)}\right)=h D_{\mu}^{*}\left(h^{-1} h_{a}{ }^{\mu}\right) \frac{\partial L_{\mathrm{M}}}{\partial\left(\mathcal{D}_{a}^{*} \varphi\right)},
$$

where we have defined $\mathcal{L}_{\mathrm{M}}=h^{-1} L_{\mathrm{M}}$ and $\bar{\partial} L_{\mathrm{M}} / \partial \varphi \equiv\left[\partial L_{\mathrm{M}}\left(\varphi, \mathcal{D}_{a}^{*} u, \ldots\right) / \partial \varphi\right]_{u=\varphi}$, so that $\varphi$ and $\mathcal{D}_{a}^{*} \varphi$ are treated as independent variables. One can develop the RHS of (65) further and put it into manifestly covariant form, by noting that

$$
h D_{\mu}^{*}\left(h^{-1} h_{a}{ }^{\mu}\right)=b^{b}{ }_{\mu}\left(\mathcal{D}_{b}^{*} h_{a}{ }^{\mu}-\mathcal{D}_{a}^{*} h_{b}{ }^{\mu}\right)=\mathcal{T}^{* b}{ }_{a b} \equiv \mathcal{T}_{a}^{*},
$$

where we have used the expression (43) for the field strength of the translational gauge field. An analogous procedure can be applied to the $\phi$ field, and so the matter equations of motion can be written in the convenient forms

$$
\begin{gathered}
\frac{\delta L_{\mathrm{M}}}{\delta \varphi}=\frac{\bar{\partial} L_{\mathrm{M}}}{\partial \varphi}-\left(\mathcal{D}_{a}^{*}+\mathcal{T}_{a}^{*}\right)\left(\frac{\partial L_{\mathrm{M}}}{\partial\left(\mathcal{D}_{a}^{*} \varphi\right)}\right)=0, \\
\frac{\delta L_{\mathrm{M}}}{\delta \phi}=\frac{\bar{\partial} L_{\mathrm{M}}}{\partial \phi}-\left(\mathcal{D}_{a}^{*}+\mathcal{T}_{a}^{*}\right)\left(\frac{\partial L_{\mathrm{M}}}{\partial\left(\mathcal{D}_{a}^{*} \phi\right)}\right)=0 .
\end{gathered}
$$

It is worth noting that these field equations, derived from the minimal-coupling procedure applied to the action $S_{\mathrm{M}}$, are not, in general, equivalent to those obtained simply by applying the minimalcoupling procedure to the field equations directly, ${ }^{9,74}$ which would be given by (67a) and (67b) without the terms containing $\mathcal{T}_{a}^{*}$. We also note that the general forms (67a) and (67b) are valid only if the action (32) has not subsequently been modified to satisfy any further required invariances that were lost in the localisation of the Weyl symmetry; this is illustrated in Section II K.

\section{Conservation laws in WGT}

The conservation laws for WGT are also easily obtained. Contrary to the approach usually adopted in the literature, it is most useful to present them in terms of quantities carrying only Latin indices. Invariance of $S_{\mathrm{G}}$ under (infinitesimal) local Lorentz (or SL(2,C)) rotations, GCTs, and local dilations, respectively, leads to the following manifestly covariant conservation laws among the contributions of the free gravitational sector to the gravitational field equations:

$$
\begin{aligned}
\left(\mathcal{D}_{c}^{*}+\mathcal{T}_{c}{ }^{*}\right)\left(h s_{a b}{ }^{c}\right)+h t_{[a b]} & =0, \\
\left(\mathcal{D}_{c}^{*}+\mathcal{T}_{c}^{*}\right)\left(h t^{c}{ }_{d}\right)-h\left(s_{a b}{ }^{c} \mathcal{R}^{a b}{ }_{c d}-t^{c}{ }_{b} \mathcal{T}^{* b}{ }_{c d}+j^{c} \mathcal{H}_{c d}\right) & =0, \\
\left(\mathcal{D}_{c}^{*}+\mathcal{T}_{c}{ }^{*}\right)\left(h j^{c}\right)-h t^{c}{ }_{c} & =0 .
\end{aligned}
$$

Similarly, the local Lorentz, GTC, and local dilation invariance properties of $S_{\mathrm{M}}$ lead, respectively, to the following manifestly covariant identities among the contributions of the matter sector to the gravitational field equations:

$$
\begin{array}{r}
\left(\mathcal{D}_{c}^{*}+\mathcal{T}_{c}^{*}\right)\left(h \sigma_{a b}{ }^{c}\right)+h \tau_{[a b]}+\frac{1}{2} \frac{\delta L_{\mathrm{M}}}{\delta \varphi} \Sigma_{a b} \varphi=0 \\
\left(\mathcal{D}_{c}^{*}+\mathcal{T}_{c}^{*}\right)\left(h \tau^{c}{ }_{d}\right)-h\left(\sigma_{a b}{ }^{c} \mathcal{R}^{a b}{ }_{c d}-\tau^{c}{ }_{b} \mathcal{T}^{* b}{ }_{c d}+\zeta^{c} \mathcal{H}_{c d}\right)+\frac{\delta L_{\mathrm{M}}}{\delta \phi} \mathcal{D}_{d}^{*} \phi+\frac{\delta L_{\mathrm{M}}}{\delta \varphi} \mathcal{D}_{d}^{*} \varphi=0 \\
\left(\mathcal{D}_{c}^{*}+\mathcal{T}_{c}^{*}\right)\left(h \zeta^{c}\right)-h \tau^{c}{ }_{c}-\frac{\delta L_{\mathrm{M}}}{\delta \phi} \phi+\frac{\delta L_{\mathrm{M}}}{\delta \varphi} w \varphi=0
\end{array}
$$

Thus, one is assured with the help of the matter field equations that the gravitational field equations become consistent. Moreover, it is worth noting that the above sets of conservation laws hold for any subset of terms in $L_{\mathrm{G}}$ and $L_{\mathrm{M}}$, respectively, that is covariant with weight $w=-4$ under local Weyl transformations. 


\section{J. Dirac matter field in WGT}

So far, we have not introduced a specific form for the matter Lagrangian. To illustrate the coupling of matter to the gravitational gauge fields, let us consider the case of a Dirac field, which will be useful for our later developments. Following our discussion in Section II A, we begin with the special-relativistic Lagrangian (14) for a classical Dirac field $\psi$ coupled to a (compensator) scalar field $\phi$. For the moment, however, we will consider only the $\psi$-dependent terms and hence omit the kinetic term for $\phi$ to obtain

$$
L_{\mathrm{D}}=\frac{1}{2} i \bar{\psi} \gamma^{\mu} \stackrel{\leftrightarrow}{\partial_{\mu}} \psi-\mu \phi \bar{\psi} \psi
$$

where $\mu$ is a dimensionless parameter, but $\mu \phi$ has the dimensions of mass in natural units. The action constructed from (70) is invariant under global Weyl transformations, provided $\psi$ and $\phi$ have the Weyl weights $w=-\frac{3}{2}$ and $w=-1$, respectively; we note that the action is also invariant under the global phase transformation $\psi \rightarrow \psi e^{i \alpha}$.

Following our general procedure for constructing a locally Weyl-invariant matter action (32) and applying it to (70), the appropriate form for the corresponding Lagrangian density is

$$
\mathcal{L}_{\mathrm{D}}=h^{-1} L_{\mathrm{D}}=h^{-1}\left(\frac{1}{2} i \bar{\psi} \gamma \gamma^{a} \stackrel{\leftrightarrow}{\mathcal{D}}_{a}^{*} \psi-\mu \phi \bar{\psi} \psi\right),
$$

where, on each $\gamma$-matrix, one has simply replaced the Greek index with a Roman one, without altering the matrix (since the $\gamma$-matrices naturally refer to the local Lorentz basis). It is clear that (71) has also inherited the invariance of the original Dirac action (70) under a global phase transformation. The first point to note is that, since both $\psi$ and $\bar{\psi}$ have Weyl weight $w=-\frac{3}{2}$, the dilation gauge field $B_{\mu}$ interacts in the same manner with each of them, thereby ruling out the interpretation of $B_{\mu}$ as the electromagnetic potential. Moreover, rather curiously, $B_{\mu}$ vanishes completely from (71). Consequently, the WGT covariant derivative operator $\mathcal{D}_{a}^{*}$ in the kinetic term in (71) can be replaced by its PGT counterpart $\mathcal{D}_{a}$, so that

$$
\mathcal{L}_{\mathrm{D}}=h^{-1}\left(\frac{1}{2} i \bar{\psi} \gamma^{a} \stackrel{\leftrightarrow}{\mathcal{D}}_{a} \psi-\mu \phi \bar{\psi} \psi\right)
$$

Put another way, the kinetic term in the Dirac action in PGT is already also invariant to local dilations.

To illustrate our general approach, however, for the moment we will consider the manifestly WGT-covariant form (71) of the Lagrangian density. Varying the corresponding action with respect to $\bar{\psi}$, one obtains a field equation of the form (67a) (with $\varphi$ replaced by $\bar{\psi}$ ), which is immediately found to read

$$
i \gamma^{a}\left(\mathcal{D}_{a}^{*}+\frac{1}{2} \mathcal{T}_{a}^{*}\right) \psi-\mu \phi \psi=0
$$

An equivalent adjoint field equation in $\bar{\psi}$ is obtained by varying the action with respect to $\psi$. Since $\mathcal{D}_{a}^{*} \psi$ and $\mathcal{T}_{a}^{*}$ transform covariantly under local dilations with weights $w=-\frac{5}{2}$ and $w=-1$, respectively, we see immediately that the LHS of (73) does indeed transform covariantly under local dilations with weight $w=-\frac{5}{2}$. We note that, as expected, the field equation (73) differs from that which would be obtained by applying the minimal coupling procedure directly at the level of the field equation; the latter would not contain the term proportional to $\mathcal{T}_{a}^{*}$. Since the Dirac Lagrangian density (71) may also be expressed as (72), however, one may use the PGT version of (67a) (obtained by removing all asterisks; see Appendix B) to show that the field equation (73) can be rewritten more simply in terms of corresponding PGT quantities as

$$
i \gamma^{a}\left(\mathcal{D}_{a}+\frac{1}{2} \mathcal{T}_{a}\right) \psi-\mu \phi \psi=0 .
$$

Although this equation is still WGT covariant, this is no longer manifest. The covariance of (74) is easily checked directly by recalling that $\mathcal{D}_{a} \psi=\mathcal{D}_{a}^{*} \psi+\frac{3}{2} \mathcal{B}_{a} \psi$ and $\mathcal{T}_{a}=\mathcal{T}_{a}^{*}-3 \mathcal{B}_{a}$ to recover (73). We note that (73) and (74) are valid for any choice of the free gravitational Lagrangian density $\mathcal{L}_{\mathrm{G}}$, but the expression for $\mathcal{T}_{a}$, or $\mathcal{T}_{a}^{*}$, will depend on the form of $\mathcal{L}_{\mathrm{G}}$.

It is of interest to rewrite (73) using the reduced covariant derivative discussed in Section II E. Substituting the expression (49) into (74) and using the fact that the Lorentz group generator 
matrices for Dirac spinors are given by $\Sigma_{a b}=\frac{1}{4}\left[\gamma_{a}, \gamma_{b}\right]$, after a short calculation one finds that

$$
i \gamma^{a}\left({ }^{0} \mathcal{D}_{a}^{*}-\frac{1}{4} \mathcal{T}_{[a b c]}^{*} \Sigma^{b c}\right) \psi-\mu \phi \psi=0 .
$$

This form of the Dirac equation is still manifestly WGT-covariant, but reveals that only the total antisymmetric part of the WGT "torsion" explicitly affects the dynamics of the Dirac field $\psi$. In other words, if $\mathcal{T}_{[a b c]}^{*}=0$ then (75) reduces to a form expressible entirely in terms of the "reduced" covariant derivative (which depends only on the $h$-field, its derivatives, and the $B$-field) and is thus equivalent to the Dirac equation obtained in the absence of torsion completely. Alternatively, one can perform the analogous procedure on (74) to obtain the form

$$
i \gamma^{a}\left({ }^{0} \mathcal{D}_{a}-\frac{1}{4} \mathcal{T}_{[a b c]} \Sigma^{b c}\right) \psi-\mu \phi \psi=0,
$$

in terms of the corresponding PGT quantities. Although (76) is not manifestly WGT-covariant, it provides the simplest expression of the dynamics of the $\psi$ field for calculational purposes and shows analogously that only the total antisymmetric part of the PGT "torsion" has an explicit effect.

From the Lagrangian density (72), we may also calculate the energy-momentum and spinangular-momentum tensors of the Dirac field $\psi$. In terms of the corresponding quantities carrying only Latin indices, one finds

$$
\begin{aligned}
h \tau^{a}{ }_{b} & =\frac{1}{2} i \bar{\psi} \gamma^{a}{\stackrel{\leftrightarrow}{\mathcal{D}_{b}} \psi} \psi \delta_{b}^{a} L_{\mathrm{D}}, \\
h \sigma_{a b c} & =\frac{1}{4} i \bar{\psi} \gamma_{[a} \gamma_{b} \gamma_{c]} \psi,
\end{aligned}
$$

where we have used the fact that $\Sigma_{a b}=\frac{1}{4}\left[\gamma_{a}, \gamma_{b}\right]$ for Dirac spinors to establish the second result. It is easily shown that the energy-momentum tensor is asymmetric and has the trace $\tau=h^{-1} \mu \phi \bar{\psi} \psi$, whereas the spin-angular-momentum tensor is totally antisymmetric, such that $\sigma_{a b c}=\sigma_{[a b c]}$. Consequently, all contractions of the spin-angular-momentum tensor vanish for Dirac matter. As one might expect from the above discussion, (77) may be rewritten in manifestly WGT-covariant form by simply making the replacement $\mathcal{D}_{a} \rightarrow \mathcal{D}_{a}^{*}$ throughout; the expression (78) is already manifestly WGT covariant.

Finally, we note that one might also consider adding the following to the matter Lagrangian density (72): kinetic and self-interaction terms for $\phi$ with Weyl weight $w=-4$ and, as discussed in Sections II B and II G, terms proportional to $\phi^{2} \mathcal{R}$ and $\phi^{2} L_{\mathcal{T}^{* 2}}$, which directly couple $\phi$ nonminimally (conformally) to the gravitational gauge field strengths, where $L_{\mathcal{T}^{* 2}}$ is given by (3), but with $\mathcal{T}_{b c}^{a}$ replaced by $\mathcal{T}^{* a}{ }_{b c}$. Thus, in the most general case, one might consider

$$
\mathcal{L}_{\mathrm{M}}=h^{-1}\left[\frac{1}{2} i \bar{\psi} \gamma^{a} \stackrel{\leftrightarrow}{\mathcal{D}}_{a} \psi-\mu \phi \bar{\psi} \psi+\frac{1}{2} v\left(\mathcal{D}_{a}^{*} \phi\right)\left(\mathcal{D}^{* a} \phi\right)-\lambda \phi^{4}-a \phi^{2} \mathcal{R}+\phi^{2} L_{\mathcal{T}^{* 2}}\right],
$$

where $\mu, v, \lambda$, and $a$ are dimensionless constants (usually positive), and there are three further

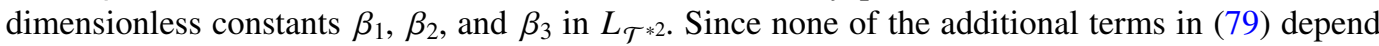
on $\psi$, the results derived above are not altered, but clearly the equation of motion for $\phi$ will become significantly more complicated.

\section{K. Electromagnetic field in WGT}

It is worthwhile also considering the coupling of the electromagnetic field to the gravitational gauge fields, since this illustrates some important differences from the Dirac field discussed above. The standard special-relativistic Lagrangian for the electromagnetic field $A_{\mu}$ (which should not be confused with the "rotational" gravitational gauge field $A^{a b}{ }_{\mu}$ ) is given by

$$
L_{\mathrm{M}}=-\frac{1}{4} F_{\mu \nu} F^{\mu \nu}-J^{\mu} A_{\mu}
$$

where the electromagnetic field strength tensor $F_{\mu \nu} \equiv \partial_{\mu} A_{v}-\partial_{v} A_{\mu}$ and $J^{\mu}$ is some source current density. We showed earlier that the action constructed from the Lagrangian (80) is invariant under global Weyl transformations, provided $w\left(A_{\mu}\right)=-1$ and $w\left(J_{\mu}\right)=-3$. Moreover, it is also invariant under the electromagnetic gauge transformation $A_{\mu} \rightarrow A_{\mu}+\partial_{\mu} \chi$, where $\chi$ is any scalar field, provided the source current density satisfies the continuity equation $\partial_{\mu} J^{\mu}=0$, which embodies the conservation of charge. 
Unlike the Dirac field, in this case the dynamical field $A_{\mu}$ carries a coordinate index. Our first task, which was unnecessary for the Dirac field, is therefore to convert this to a Roman index by constructing the covariant field $\mathcal{A}_{a}=h_{a}{ }^{\mu} A_{\mu}$. Similarly, we introduce the covariant current density $\mathcal{J}_{a}=h_{a}{ }^{\mu} J_{\mu}$. This leads us, however, to a subtle point. In the special relativistic case (80), the field components can equally well refer to the Cartesian coordinate basis $\boldsymbol{e}_{\mu}$ or the local Lorentz basis $\hat{\boldsymbol{e}}_{a}$, which can be chosen to coincide. In principle, however, the field components should properly refer to the latter. Thus, once the Weyl transformation is localised, for consistency one requires the covariant fields to have the Weyl weights $w\left(\mathcal{A}_{a}\right)=-1$ and $w\left(\mathcal{J}_{a}\right)=-3$; consequently, the fields carrying a Greek index have weights $w\left(A_{\mu}\right)=0$ and $w\left(J_{\mu}\right)=-2$.

Following the general procedure for constructing a locally Weyl-invariant matter action (32) leads to the Lagrangian density

$$
\mathcal{L}_{\mathrm{M}}=h^{-1} L_{\mathrm{M}}=-h^{-1}\left(\frac{1}{4} \widehat{\mathcal{F}}_{a b}^{*} \widehat{\mathcal{F}}^{* a b}+\mathcal{J}^{a} \mathcal{A}_{a}\right),
$$

where we have defined $\widehat{\mathcal{F}}_{a b}^{*} \equiv \mathcal{D}_{a}^{*} \mathcal{A}_{b}-\mathcal{D}_{b}^{*} \mathcal{A}_{a}$. Since $w\left(\widehat{\mathcal{F}}_{a b}^{*}\right)=-2, \mathcal{L}_{\mathrm{M}}$ has an overall Weyl weight of zero, as required. Unfortunately, the Lagrangian density (81) is inappropriate for describing the coupling of the electromagnetic and gravitational gauge fields, since the corresponding action does not inherit the necessary invariance under electromagnetic gauge transformations. This is easily seen by noting that

$$
\widehat{\mathcal{F}}_{a b}^{*}=\mathcal{F}_{a b}-\mathcal{T}^{* c}{ }_{a b} \mathcal{A}_{c},
$$

where we have defined $\mathcal{F}_{a b} \equiv h_{a}{ }^{\mu} h_{b}{ }^{\nu} F_{\mu \nu}$, in which $F_{\mu \nu} \equiv D_{\mu}^{*} A_{\nu}-D_{v}^{*} A_{\mu}=\partial_{\mu} A_{\nu}-\partial_{\nu} A_{\mu}$, and $\mathcal{T}^{* c}{ }_{a b}$ is the WGT field strength tensor of the $h$ gravitational gauge field, as defined in (42). It is apparent that $\mathcal{F}_{a b}$ does inherit the original electromagnetic gauge invariance of $F_{\mu \nu}$, whereas the second term on the RHS of (82) is clearly not invariant under the electromagnetic gauge transformation, and so neither is $\widehat{\mathcal{F}}_{a b}^{*}$. Moreover, $\mathcal{F}_{a b}$ has a Weyl weight $w\left(\mathcal{F}_{a b}\right)=-2$. Thus, substituting (82) into (81) and discarding the offending terms, we arrive instead at the Lagrangian density

$$
\mathcal{L}_{\mathrm{M}}=h^{-1} L_{\mathrm{M}}=-h^{-1}\left(\frac{1}{4} \mathcal{F}_{a b} \mathcal{F}^{a b}+\mathcal{J}^{a} \mathcal{A}_{a}\right),
$$

which has an overall Weyl weight of zero, as required, provided $w\left(\mathcal{J}_{a}\right)=-3$. It is worth noting that this form for $\mathcal{L}_{\mathrm{M}}$ is identical to that obtained in PGT, as should be clear from the above discussion. Hence, as we found for the Dirac field, the action for the electromagnetic field in PGT is already invariant under local changes of scale. Note that $\mathcal{L}_{\mathrm{M}}$ does not depend on the $A$ or $B$ gauge fields.

The equation of motion for the electromagnetic field is given by $\delta \mathcal{L}_{\mathrm{EM}} / \delta A_{\mu}=0$, which is easily shown to be equivalent to $\delta \mathcal{L}_{\mathrm{EM}} / \delta \mathcal{A}_{a}=0$, and in manifestly WGT-covariant form this reads

$$
\left(\mathcal{D}_{a}^{*}+\mathcal{T}_{a}^{*}\right) \mathcal{F}^{a c}-\frac{1}{2} \mathcal{T}^{* c}{ }_{a b} \mathcal{F}^{a b}=\mathcal{J}^{c} .
$$

It is worth noting that this equation does not have the generic form (67a), owing to the term containing $\mathcal{T}^{* c}{ }_{a b}$ on the LHS. This additional term arises because the demands of electromagnetic gauge invariance of the action dictated the use of the Lagrangian density (83), rather than (81). Had we (erroneously) used the latter, the resulting equation of motion would not have included this extra term and would indeed have taken the general form (67a). We note that the electromagnetic field strength tensor also satisfies a differential (Bianchi) identity, which is easily expressible in manifestly WGT-covariant form as

$$
\mathcal{D}_{[a}^{*} \mathcal{F}_{b c]}-\mathcal{T}_{[a b}^{* d} \mathcal{F}_{c] d}=0 .
$$

Note that all contractions of this identity are trivial.

Since the Lagrangian (83) is identical to that in PGT, one immediately finds that the equation of motion (84) and differential identity (85) satisfied by the electromagnetic field can be rewritten in terms of corresponding PGT quantities by simply removing asterisks as

$$
\begin{aligned}
\left(\mathcal{D}_{a}+\mathcal{T}_{a}\right) \mathcal{F}^{a c}-\frac{1}{2} \mathcal{T}^{c}{ }_{a b} \mathcal{F}^{a b} & =\mathcal{J}^{c}, \\
\mathcal{D}_{[a} \mathcal{F}_{b c]}-\mathcal{T}^{d}{ }_{[a b} \mathcal{F}_{c] d} & =0 .
\end{aligned}
$$


The covariance of these equations under local dilations is easily checked directly by noting that $\mathcal{D}_{a} \mathcal{F}^{a c}=\left(\mathcal{D}_{a}^{*}+2 \mathcal{B}_{a}\right) \mathcal{F}^{a c}$ and $\mathcal{T}_{a}=\mathcal{T}^{*}{ }_{a}-3 \mathcal{B}_{a}$, which on substitution recovers (84) and (85).

As we did for the Dirac equation in Sec. II J, it is interesting to rewrite the electromagnetic field equation (84) and Bianchi identity (85) in terms of the reduced covariant derivative discussed in Section II E. Thus, substituting (49) into (84) and (85), one may show straightforwardly that the electromagnetic field equation and Bianchi identity become, respectively,

$$
{ }^{0} \mathcal{D}_{a}^{*} \mathcal{F}^{a c}=\mathcal{J}^{c} \quad \text { and } \quad{ }^{0} \mathcal{D}_{[a}^{*} \mathcal{F}_{b c]}=0 .
$$

These equations are still manifestly WGT-covariant, but have precisely the same forms as would be obtained in the absence of torsion. Thus, irrespective of the nature of the WGT torsion, it plays no explicit role in the dynamics of the electromagnetic field. Alternatively, one can perform an analogous procedure to rewrite (86) and (87) in terms of the reduced PGT covariant derivative as

$$
{ }^{0} \mathcal{D}_{a} \mathcal{F}^{a c}=\mathcal{J}^{c} \quad \text { and } \quad{ }^{0} \mathcal{D}_{[a} \mathcal{F}_{b c]}=0,
$$

which are identical to (88) with all asterisks removed. Although not manifestly WGT-covariant, the Equations (89) provide the simplest expression of the dynamics of the electromagnetic field for calculational purposes and show that the PGT torsion has no direct effect.

To complete the description of the electromagnetic field in WGT, we derive its energymomentum and spin-angular-momentum tensors. Setting $\mathcal{J}^{a}=0$ in (83), one finds

$$
h \tau^{a}{ }_{b}=\frac{1}{4} \delta_{b}^{a} \mathcal{F}^{c d} \mathcal{F}_{c d}-\mathcal{F}^{a c} \mathcal{F}_{b c}
$$

whereas $\sigma_{a b}{ }^{c}=0$ immediately. We see that $\tau_{b}^{a}$ is the natural generalisation of the standard energy-momentum tensor for the electromagnetic field in the absence of gravity. Moreover, $\tau_{a b}$ is symmetric and traceless, as one would expect for a field with vanishing spin-angular-momentum and zero mass. We note that the expression (90) is already manifestly WGT (and PGT) covariant.

Finally, one may straightforwardly include the interaction of the electromagnetic and Dirac fields, both of which are coupled to gravity, by noting that the covariant current density of the Dirac field is simply $\mathcal{J}^{a}=q \bar{\psi} \gamma^{a} \psi$, which has the required Weyl weight $w\left(\mathcal{J}^{a}\right)=-3$. Thus, one simply makes this substitution into (83) and adds the result to (79).

\section{Einstein gauge and scale-invariant variables}

As mentioned in the Introduction, one may use the scale gauge freedom to set the scalar field $\phi$ to a constant (provided $\phi \neq 0$ ), which is known as the Einstein gauge. Although it is most common simply to set $\phi(x)=1$, this can be misleading and so we instead set $\phi(x)=\phi_{0}$, where $\phi_{0}$ is a constant with the same units as $\phi$. Adopting the Einstein gauge can be viewed simply as a mathematical convenience, but it is often also given the physical interpretation of corresponding to some spontaneous breaking of the scale symmetry. Indeed, it is usually considered that setting $\phi=\phi_{0}$ represents the choice of some definite scale in the theory, thereby breaking scale-invariance.

The forms of the field equations in WGT, which we discussed in general in Section II H, can be simplified considerably by adopting the Einstein gauge. There is, however, an alternative approach, which is not usually followed in the literature, where one recasts the theory in terms of scale-invariant field variables. ${ }^{50}$ We show here that, for any WGT Lagrangian density of the form considered in (60), this procedure yields equations of motion in the new field variables that are identical in form to those obtained after setting $\phi(x)=\phi_{0}$ in the equations of motion for the original fields. This therefore provides a rather different interpretation of the Einstein gauge than that traditionally assumed, since the approach using scale-invariant variables involves no breaking of the scale symmetry. Indeed, one may regard adopting the Einstein gauge merely as a shortcut to obtaining the forms of the equations of motion for the scale-invariant field variables. 
To demonstrate this equivalence, we begin by introducing the new matter and gravitational gauge fields

$$
\begin{aligned}
\widehat{\varphi} & \equiv\left(\frac{\phi}{\phi_{0}}\right)^{-w} \varphi, \\
\widehat{h}_{a}^{\mu} & \equiv\left(\frac{\phi}{\phi_{0}}\right)^{-1} h_{a}{ }^{\mu}, \\
\widehat{A}_{\mu}^{a b} & \equiv A^{a b}, \\
\widehat{B}_{\mu} & \equiv B_{\mu}-\partial_{\mu} \ln \left(\frac{\phi}{\phi_{0}}\right),
\end{aligned}
$$

where $w$ is the Weyl weight of the original matter field $\varphi$. It is straightforward to show that each of these new variables transforms covariantly with Weyl weight $w=0$ under local dilations, and is hence scale-invariant. It is also convenient to define the further scale-invariant variables $\widehat{\mathcal{A}}^{a b}{ }_{c} \equiv \widehat{h}_{a}{ }^{\mu} \widehat{A}^{a b}{ }_{\mu}$ and $\widehat{\mathcal{B}}_{a} \equiv \widehat{h}_{a}^{\mu} \widehat{B}_{\mu}$.

One immediately finds that the covariant derivative of the scalar field $\phi$ may be written as $\mathcal{D}_{a}^{*} \phi=-\left(\phi^{2} / \phi_{0}\right) \widehat{\mathcal{B}}_{a}$, whereas the covariant derivative of some general field $\chi$ with Weyl weight $w$ may be written as

$$
\begin{aligned}
\mathcal{D}_{a}^{*} \chi & \equiv\left(\mathcal{D}_{a}+w \mathcal{B}_{a}\right) \chi \\
& =\left(\frac{\phi}{\phi_{0}}\right)^{1-w}\left(\widehat{\mathcal{D}}_{a}+w \widehat{\mathcal{B}}_{a}\right) \widehat{\chi} \equiv\left(\frac{\phi}{\phi_{0}}\right)^{1-w} \widehat{\mathcal{D}}_{a}^{*} \widehat{\chi},
\end{aligned}
$$

where $\widehat{\chi}=\left(\phi / \phi_{0}\right)^{-w} \chi$ and we have defined the derivative operators $\widehat{\mathcal{D}}_{a} \equiv \widehat{h}_{a}^{\mu} D_{\mu}$ and $\widehat{\mathcal{D}}_{a}^{*}=\widehat{\mathcal{D}}_{a}+$ $w \widehat{\mathcal{B}}_{a}$, the latter of which preserves the Weyl weight of the quantity on which it acts. From (92), one sees that, aside from the overall multiplicative factor $\left(\phi / \phi_{0}\right)^{1-w}$, the scale-invariant quantity $\widehat{\mathcal{D}}_{a}^{*} \widehat{\chi}$ has the same functional dependency on $\widehat{\chi}, \widehat{h}_{a}^{\mu}, \widehat{A}^{a b}{ }_{\mu}$, and $\widehat{B}_{\mu}$, respectively, as $\mathcal{D}_{a}^{*} \chi$ does on $\chi, h_{a}{ }^{\mu}$, $A^{a b}{ }_{\mu}$, and $B_{\mu}$. Moreover, one also quickly finds that the WGT gauge field strengths defined in (41) may be written as $\mathcal{R}^{a b}{ }_{c d}=\left(\phi / \phi_{0}\right)^{2} \widehat{\mathcal{R}}^{a b}{ }_{c d}, \mathcal{T}^{* a}{ }_{b c}=\left(\phi / \phi_{0}\right) \widehat{\mathcal{T}}^{* a}{ }_{b c}$, and $\mathcal{H}_{a b}=\left(\phi / \phi_{0}\right)^{2} \widehat{\mathcal{H}}_{a b}$, where each quantity with a caret is scale-invariant and has the same functional dependence on $\widehat{h}_{a}^{\mu}, \widehat{A}_{\mu}^{a b}$, and $\widehat{B}_{\mu}$ as the corresponding original quantity does on $h_{a}{ }^{\mu}, A^{a b}{ }_{\mu}$, and $B_{\mu}$, respectively.

Noting that $h^{-1}=\left(\phi / \phi_{0}\right)^{-4} \widehat{h}^{-1}$, one is thus led to the important conclusion that the Lagrangian density $\mathcal{L}_{\mathrm{T}}$ in (60) may be written as (suppressing indices for brevity)

$$
\mathcal{L}_{\mathrm{T}}=\mathcal{L}_{\mathrm{G}}(\widehat{h}, \widehat{A}, \partial \widehat{A}, \partial \widehat{B})+\mathcal{L}_{\mathrm{M}}\left(\widehat{\varphi}, \partial \widehat{\varphi}, \phi_{0}, 0, \widehat{h}, \partial \widehat{h}, \widehat{A}, \partial \widehat{A}, \widehat{B}\right) .
$$

In other words, when written in terms of the scale-invariant field variables, the Lagrangian density $\mathcal{L}_{\mathrm{T}}$ (indeed each term separately) has the same functional form as it does in terms of the original variables with $\phi=\phi_{0}$. Thus, if $\chi$ represents $\varphi, h_{a}{ }^{\mu}, A^{a b}{ }_{\mu}$, or $B_{\mu}$, one may immediately conclude that (each term in) the equation of motion $\delta \mathcal{L}_{\mathrm{T}} / \delta \widehat{\chi}=0$ has the same functional form as (the corresponding term in) $\delta \mathcal{L}_{\mathrm{T}} /\left.\delta \chi\right|_{\phi=\phi_{0}}=0$, but with $\varphi, h_{a}{ }^{\mu}, A^{a b}{ }_{\mu}$, and $B_{\mu}$ replaced by their scale-invariant counterparts.

For the $\phi$-field equation, however, the situation is more subtle, since (93) does not depend explicitly on $\phi$. Nonetheless, one can still show that the same equivalence holds as follows. Working first in terms of the original variables, one begins by noting that $\delta \mathcal{L}_{\mathrm{T}} / \delta \phi=\delta \mathcal{L}_{\mathrm{M}} / \delta \phi$, since $\mathcal{L}_{\mathrm{G}}$ does not depend on $\phi$ or its derivative. As discussed in Section II I, the invariance of the matter action $S_{\mathrm{M}}$ under local dilations leads to the WGT conservation law (69c), which may be straightforwardly rewritten as

$$
\left.\phi_{0} \frac{\delta \mathcal{L}_{\mathrm{M}}}{\delta \phi}\right|_{\phi=\phi_{0}}=-\left.h_{a}{ }^{\mu} \frac{\delta \mathcal{L}_{\mathrm{M}}}{\delta h_{a}{ }^{\mu}}\right|_{\phi=\phi_{0}}+\partial_{\mu}\left(\frac{\delta \mathcal{L}_{\mathrm{M}}}{\delta B_{\mu}}\right)_{\phi=\phi_{0}},
$$

where we have made use of the $\varphi$-field equation $\delta \mathcal{L}_{\mathrm{T}} / \delta \varphi=\delta \mathcal{L}_{\mathrm{M}} / \delta \varphi=0$ and set $\phi=\phi_{0}$ in the resulting expression. Turning now to the expression (93) for $\mathcal{L}_{\mathrm{T}}$ in terms of scale-invariant variables 
and applying the chain rule for partial derivatives, one may show after a lengthy but straightforward calculation that

$$
\phi \frac{\delta \mathcal{L}_{\mathrm{M}}}{\delta \phi}=-\widehat{h}_{a}^{\mu} \frac{\delta \mathcal{L}_{\mathrm{M}}}{\delta \widehat{h}_{a}^{\mu}}+\partial_{\mu}\left(\frac{\partial \mathcal{L}_{\mathrm{M}}}{\partial \widehat{B}_{\mu}}\right),
$$

where we have again made use of the $\varphi$-field equation. Since $\mathcal{L}_{\mathrm{M}}$ does not depend on the derivatives of $\widehat{B}_{\mu}$, one may replace $\partial \mathcal{L}_{\mathrm{M}} / \partial \widehat{B}_{\mu}$ by $\delta \mathcal{L}_{\mathrm{M}} / \delta \widehat{B}_{\mu}$ in the above equation. Then, comparing (94) and (95) and making use of our earlier observations regarding the other field equations, one sees that the $\phi$-field equation written in terms of the scale-invariant variables also has the same functional form as the $\phi$-field equation in terms of the original variables with $\phi=\phi_{0}$.

For the remainder of our discussion, we will continue to use the original field variables for the most part, and occasionally simplify field equations by adopting the Einstein gauge $\phi(x)=\phi_{0}$, since this approach is more familiar and straightforward. Nonetheless, the equivalence of the results so obtained with those expressed in terms of scale-invariant variables should be borne in mind throughout.

\section{Motion of test particles in WGT}

We may determine the equation of motion of a massive matter test particle in WGT from the Dirac Lagrangian (12), but modified to accommodate local scale-invariance by simply introducing the massless scalar field $\phi$ and making the replacement $m \rightarrow \mu \phi$, as in (70). One begins by constructing the appropriate action for a spin- $\frac{1}{2}$ point particle and then makes the full classical approximation in which the particle spin is neglected. This procedure is outlined for a free particle in Appendix A. To include gravitational effects in the point particle action (A9), one needs only to make the replacements $p_{\mu} \rightarrow p_{a} \equiv h_{a}{ }^{\mu} p_{\mu}, \dot{x}^{\mu} \rightarrow v^{a} \equiv b^{a}{ }_{\mu} \dot{x}^{\mu}$, and $m \rightarrow \mu \phi$, which gives ${ }^{75}$

$$
S=-\int d \lambda\left[p_{a} v^{a}-\frac{1}{2} e\left(p_{a} p^{a}-\mu^{2} \phi^{2}\right)\right],
$$

where the three dynamical variables are the particle 4-momentum $p^{a}(\lambda), 4$-velocity $v^{a}(\lambda)$, and the einbein $e(\lambda)$ along the worldline, which is parameterised by $\lambda$. We note that the Weyl weights of the quantities appearing in (96) are $w\left(p^{a}\right)=-1, w\left(v^{a}\right)=0, w(e)=1, w(\lambda)=1$, and $w(\phi)=-1$, so that the action is indeed scale-invariant.

Varying the action (96) with respect to the three dynamical variables, respectively, one obtains the equations of motion

$$
\begin{aligned}
v^{a} & =e p^{a}, \\
\dot{p}_{a} & =c^{c}{ }_{a b} v^{b} p_{c}+e \mu^{2} \phi \partial_{a} \phi, \\
p^{2} & =\mu^{2} \phi^{2},
\end{aligned}
$$

where $p^{2} \equiv p_{a} p^{a}$ and the quantities $c^{c}{ }_{a b}$ are the PGT counterparts of those defined in (45) (obtained by removing all asterisks). It is straightforward to show that (98) can be written in a manifestly WGT-covariant manner as

$$
v^{c}\left(\mathcal{D}_{c}^{*} p_{a}-\mathcal{T}_{\text {cab }}^{*} p^{b}\right)=e \mu^{2} \phi \mathcal{D}_{a}^{*} \phi .
$$

At first sight, (100) appears to show that "torsion" enters the particle equation of motion directly. As one might suspect from our discussion in Sections II J and II K, however, appearances can be deceptive. Indeed, one may show directly that (100) can be rewritten as

$$
v^{c 0} \mathcal{D}_{c}^{*} p_{a}=e \mu^{2} \phi^{0} \mathcal{D}_{a}^{*} \phi,
$$

where ${ }^{0} \mathcal{D}_{c}^{*}$ is the reduced WGT covariant derivative, defined in (48). The equation of motion (101) is again manifestly WGT-covariant, but takes its simplest form when one chooses $e=1 /(\mu \phi)$, in which case $v^{2}=1$ and $\lambda$ corresponds to the proper time $\tau$ along the worldline; in this case the equation of motion becomes

$$
\phi v^{b 0} \mathcal{D}_{b}^{*} v_{a}=\left(\delta_{a}^{b}-v_{a} v^{b}\right)^{0} \mathcal{D}_{b}^{*} \phi
$$


For ease of calculation, one can rewrite this equation in terms of the reduced PGT-covariant derivative as ${ }^{76-78}$

$$
\phi v^{b 0} \mathcal{D}_{b} v_{a}=\left(\delta_{a}^{b}-v_{a} v^{b}\right) \partial_{b} \phi
$$

which is again WGT covariant, but not manifestly so. Moreover, if one uses local scale invariance to impose the Einstein gauge $\phi=\phi_{0}$ (a constant), then (103) reduces to

$$
v^{b 0} \mathcal{D}_{b} v_{a}=0 .
$$

When reinterpreted geometrically (see Section II O and Appendix B), the quantities ${ }^{0} A^{a b}{ }_{\mu}$ appearing in ${ }^{0} \mathcal{D}_{b}$ contain the same geometrical information as the metric connection. Thus, (104) describes the gauge theory equivalent of geodesic motion (as opposed, for example, to motion along autoparallels, which would correspond to the equation of motion $v^{b} \mathcal{D}_{b} v_{a}=0$ ). We note that this conclusion differs from some previous studies, ${ }^{79,80}$ in which the WGT conservation laws applied to the energy momentum tensor of dust yield an equation of motion for a matter test particle that contains an additional electromagnetic-like Lorentz force term proportional to $\left(q_{\mathrm{w}} / m\right) \mathcal{H}_{a b} v^{b}$, where $q_{\mathrm{W}}$ is the particle's Weyl charge and $m$ is its mass. Such a term is absent from the equation of motion (104), since the latter is derived by considering the Dirac action, which does not depend on the dilation gauge field $B_{\mu}$. It is feasible that by constructing a point particle action based instead upon (say) the action for a scalar field, which does depend on the dilation gauge field, one would arrive at an equation of motion that does contain the Lorentz-force term above. We do not consider this further here, however, since in our opinion "normal" matter is more appropriately described by Dirac particles.

By setting $\mu=0$ in the action (96) and choosing the einbein $e=1$, such that $v^{2}=0$, one finds that the equation of motion in the massless case (for example, a massless neutrino) is again given by (104), even without imposing the Einstein gauge. One may also arrive at a similar conclusion for the motion of photons by directly considering the dynamics of the electromagnetic field. As discussed in Section II K, the EM field tensor in WGT again satisfies the field equation and Bianchi identity given in (89), which have precisely the same form as those obtained in the absence of torsion. Consequently, one may immediately infer that the equation of motion for photons is also given by (104), so that they too follow the gauge theory equivalent of geodesic motion. It is worth noting that the lack of a Lorentz-force term is again related to the absence of the dilation gauge field $B_{\mu}$ from the WGT action for the electromagnetic field.

To deduce the geodesic equation of motion (104), it was necessary to impose the Einstein gauge condition $\phi=\phi_{0}$. As one might suspect from our discussion in Sec. II L, however, this is unnecessary and an equivalent result may be obtained directly in terms of scale-invariant variables, as we now demonstrate. Returning to the action (96), let us introduce the new scale-invariant variables

$$
\begin{aligned}
\widehat{\lambda} & \equiv\left(\frac{\phi}{\phi_{0}}\right) \lambda, \\
\widehat{p}_{a} & \equiv \widehat{h}_{a}^{\mu} p_{\mu}=\left(\frac{\phi}{\phi_{0}}\right)^{-1} p_{a}, \\
\widehat{v}^{a} & \equiv \widehat{b}^{a}{ }_{\mu} \frac{d x^{\mu}}{d \widehat{\lambda}}=v^{a}, \\
\widehat{e} & \equiv\left(\frac{\phi}{\phi_{0}}\right) e,
\end{aligned}
$$

where $\widehat{p}_{a}, \widehat{v}^{a}$, and $\widehat{e}$ are to be considered as functions of $\widehat{\lambda}$, such that, for example, $\widehat{p}_{a}(\widehat{\lambda}) \equiv$ $\widehat{h}_{a}^{\mu} p_{\mu}(\lambda(\widehat{\lambda}))$. It is then straightforward to show that (96) can be rewritten as

$$
S=-\int d \widehat{\lambda}\left[\widehat{p}_{a} \widehat{v}^{a}-\frac{1}{2} \widehat{\epsilon}\left(\widehat{p}_{a} \widehat{p}^{a}-\mu^{2} \phi_{0}^{2}\right)\right],
$$

where we have introduced the new scale-invariant einbein $\widehat{\epsilon}=\left[1-\widehat{\lambda}\left(d x^{\mu} / d \widehat{\lambda}\right) \partial_{\mu} \ln \left(\phi / \phi_{0}\right)\right] \widehat{e}$. Thus, in terms of the scale-invariant variables $\widehat{\lambda}, \widehat{p}_{a}, \widehat{v}^{a}$, and $\widehat{\epsilon}$, respectively, the action (106) has the same 
functional form as it does in terms of the original variables $\lambda, p_{a}, v^{a}$, and $e$ with $\phi=\phi_{0}$. Moreover, in the definitions of the scale-invariant variables (105), each occurrence of the gravitational gauge field $h_{a}{ }^{\mu}$ or its inverse $b^{a}{ }_{\mu}$ in the original variables has merely been replaced by its scale-invariant counterpart $\widehat{h}_{a}^{\mu}$ or $\widehat{b}_{\mu}^{a}$. Thus, the calculation presented above follows through in a similar manner, but in terms of the corresponding scale-invariant variables and with $\phi=\phi_{0}$. One therefore arrives at a result analogous to (104) but written entirely in terms of scale-invariant variables, namely,

$$
\widehat{v}^{b 0} \widehat{\mathcal{D}}_{b} \widehat{v}_{a}=0
$$

where we have defined the derivative operator ${ }^{0} \widehat{\mathcal{D}}_{a} \equiv \widehat{h}_{a}^{\mu}{ }^{\mu} \mathcal{D}_{\mu}$.

\section{N. Reduced WGT}

In Section II E, we introduced the "reduced" WGT covariant derivative operator ${ }^{0} D_{\mu}^{*}$ in (48), to which the "full" WGT covariant derivative (20) reduces in the case that the WGT torsion $\mathcal{T}_{a b c}^{*}$ vanishes (which is a properly WGT-covariant condition). In the context of WGT, we will use the term "reduced" to refer to versions of quantities in which, by construction, the rotational gauge field is not an independent field, but is determined by the other gauge fields $h$ and $B$ through the expression (46) with $\mathcal{T}_{a b c}^{*} \equiv 0$. Hence such quantities may be written entirely in terms of these other gauge fields and are denoted by a zero superscript preceding the kernel letter.

One can use the covariant derivative ${ }^{0} D_{\mu}^{*}$ to build an alternative class of scale-invariant gravitational gauge theories, which we term "reduced WGT," that depend only on the $h$ and $B$ gravitational gauge fields and correspond mathematically to imposing the condition of vanishing WGT torsion directly at the level of the action. Such theories have been studied previously, most notably by Dirac, ${ }^{54}$ and are of some phenomenological interest.

One begins by defining the "reduced" gauge field strengths in the usual manner by considering the commutator of reduced covariant derivatives. One finds that

$$
\left[{ }^{0} D_{\mu}^{*},{ }^{0} D_{\nu}^{*}\right] \varphi=\frac{1}{2}{ }^{*} R^{* a b}{ }_{\mu \nu} \Sigma_{a b} \varphi+w H_{\mu \nu} \varphi,
$$

where we have defined the "reduced" field strength tensor ${ }^{0} R^{* a b}{ }_{\mu \nu}\left(h, \partial h, \partial^{2} h, B, \partial B\right)$, which is again given by the formula (39), but with $A^{a b}{ }_{\mu}$ replaced by ${ }^{0} A^{* a b}{ }_{\mu}$, and hence depends only on the $h$ and $B$ fields and their derivatives, as indicated. Considering instead the commutator of "reduced" generalised covariant derivatives, one obtains

$$
\left[{ }^{0} \mathcal{D}_{c}^{*},{ }^{0} \mathcal{D}_{d}^{*}\right] \varphi=\frac{1}{2}{ }^{0} \mathcal{R}^{* a b}{ }_{c d} \Sigma_{a b} \varphi+w \mathcal{H}_{c d} \varphi
$$

where ${ }^{0} \mathcal{R}^{* a b}{ }_{c d}=h_{c}{ }^{\mu} h_{d}{ }^{\mu 0} R^{* a b}{ }_{\mu \nu}$. Unlike (41), the commutator (109) has no term containing a "reduced" translational field strength of the $h$ gauge field, since ${ }^{0} \mathcal{T}^{* a}{ }_{b c} \equiv h_{b}{ }^{\mu} h_{c}{ }^{\nu}\left({ }^{0} D_{\mu}^{*} b^{a}{ }_{v}-\right.$ $\left.{ }^{0} D_{v}^{*} b^{a}{ }_{\mu}\right)=0$. As one might expect, the Bianchi identities satisfied by the reduced field strength tensors are identical to those given in Section II F, but with the replacements $\mathcal{D}_{a}^{*} \rightarrow{ }^{0} \mathcal{D}_{a}^{*}, \mathcal{R}_{a b c d} \rightarrow{ }^{0} \mathcal{R}_{a b c d}^{*}$ and $\mathcal{T}_{a b c}^{*} \rightarrow{ }^{0} \mathcal{T}_{a b c}^{*} \equiv 0$.

Since the "full" generalised covariant derivative is given in terms of the "reduced" one by (49), it is straightforward to show that the "full" field strength tensor $\mathcal{R}^{a b}{ }_{c d}$ appearing in (41) is related to its "reduced" counterpart by

$$
\begin{aligned}
\mathcal{R}^{a b}{ }_{c d} & ={ }^{0} \mathcal{R}^{* a b}{ }_{c d}+{ }^{0} \mathcal{D}_{c}^{*} \mathcal{K}^{* a b}{ }_{d}-{ }^{0} \mathcal{D}_{d}^{*} \mathcal{K}^{* a b}{ }_{c}+\mathcal{K}^{* a}{ }_{e c} \mathcal{K}^{* e b}{ }_{d}-\mathcal{K}^{* a}{ }_{e d} \mathcal{K}^{* e b}{ }_{c}, \\
\mathcal{R}^{a}{ }_{c} & ={ }^{0} \mathcal{R}^{* a}{ }_{c}+{ }^{0} \mathcal{D}_{c}^{*} \mathcal{K}^{* a b}{ }_{b}-{ }^{0} \mathcal{D}_{b}^{*} \mathcal{K}^{* a b}{ }_{c}+\mathcal{K}^{* a}{ }_{e c} \mathcal{K}^{* e b}{ }_{b}-\mathcal{K}^{* a}{ }_{e b} \mathcal{K}^{* e b}{ }_{c}, \\
\mathcal{R} & ={ }^{0} \mathcal{R}^{*}+\frac{1}{4} \mathcal{T}^{* a b c} \mathcal{T}_{a b c}{ }^{*}+\frac{1}{2} \mathcal{T}^{* a b c} \mathcal{T}_{b a c}{ }^{*}-\mathcal{T}^{* a} \mathcal{T}_{a}{ }^{*}-2^{0} \mathcal{D}_{a}^{*} \mathcal{T}^{* a},
\end{aligned}
$$

where, for completeness, we have also given explicitly the relationships between the contractions of $\mathcal{R}^{a b}{ }_{c d}$ and ${ }^{0} \mathcal{R}^{* a b}{ }_{c d}$.

For reduced WGT, the free gravitational Lagrangian density $\mathcal{L}_{\mathrm{G}}=h^{-1} L_{\mathrm{G}}$, where by analogy with (58),

$$
L_{\mathrm{G}}=L_{0_{\mathcal{R}^{* 2}}}+L_{\mathcal{H}^{2}},
$$


which is based on (4) with $\mathcal{R}^{a b}{ }_{c d}$ replaced by ${ }^{0} \mathcal{R}^{* a b}{ }_{c d}$. As previously, one can simplify (111) still further (in $D \leq 4$ dimensions), since ${ }^{0} \mathcal{R}_{a b c d}^{*}$ and its contractions also satisfy a Gauss-Bonnet identity of the form (59), but with $\mathcal{R}_{a b c d} \rightarrow{ }^{0} \mathcal{R}_{a b c d}^{*}$. Thus, one can set to zero any one of the parameters $\alpha_{i}$ in (111) with no loss of generality (at least classically). A typical form for the matter Lagrangian density $\mathcal{L}_{\mathrm{M}}$ is that given in (79), but with appropriate modifications, ${ }^{81}$ namely,

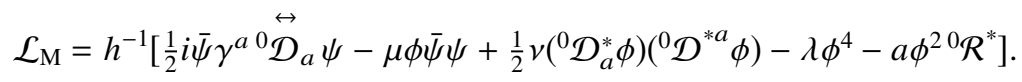

The total Lagrangian density thus has the following functional dependencies in the most general case:

$$
\mathcal{L}_{\mathrm{T}}=\mathcal{L}_{\mathrm{G}}\left(h, \partial h, \partial^{2} h, B, \partial B\right)+\mathcal{L}_{\mathrm{M}}\left(\varphi, \partial \varphi, \phi, \partial \phi, h, \partial h, \partial^{2} h, B, \partial B\right) .
$$

The resulting field equations for the gravitational gauge fields will clearly have the same generic structure as those given in Section II $\mathrm{H}$, although without the $A$-field equation (62b), but the specific forms for each term are not, in general, obtained from the corresponding "full" WGT expressions simply by replacing the "full" covariant derivative and field strength tensors with their "reduced" counterparts. Nonetheless, the matter field equations will still take the form (67a) and (67b), but with the replacements $\mathcal{D}_{a}^{*} \rightarrow{ }^{0} \mathcal{D}_{a}^{*}$ and $\mathcal{T}_{a}^{*} \rightarrow{ }^{0} \mathcal{T}_{a}{ }^{*} \equiv 0$. One should also note, however, that the gravitational and matter Lagrangians are both, in general, quadratic in second derivatives of the $h$-field, and so the resulting field equations are typically linear in fourth-order derivatives of $h$; such theories typically suffer from Ostrogradsky's instability, although this needs to be investigated on a case-by-case basis.

The conservation laws in reduced WGT do have the same form as those given in Section II I for WGT, but with the replacements $\mathcal{D}_{a}^{*} \rightarrow{ }^{0} \mathcal{D}_{a}^{*}, \mathcal{T}_{a b c}^{*} \rightarrow{ }^{0} \mathcal{T}_{a b c}^{*} \equiv 0$, and $s_{a b c} \equiv 0 \equiv \sigma_{a b c}$. Furthermore, by performing analogous calculations to those presented in Sections II L and II $\mathrm{M}$, respectively, one may show that our conclusions in WGT regarding the interpretation of the Einstein gauge and the motion of test particles also apply in reduced WGT.

Finally, as alluded to above, it is important to distinguish reduced WGT, in which the condition of vanishing WGT torsion is imposed directly at the level of the action, from instead setting the (properly WGT-covariant) condition $\mathcal{T}_{a b c}^{*}=0$ in the field equations of WGT. In the latter case, the notion of the reduced covariant derivative operator ${ }^{0} D_{\mu}^{*}$ in (48) is still useful, as we have seen in Sections II J, II K, and II M, but the basic field equations remain linear in secondorder derivatives of the gauge fields; although one can substitute for the rotational gauge field to obtain equations that contain higher-order derivatives, the theory does not suffer from Ostrogradsky's instability. In essence, the two approaches correspond to "second-order" and "first-order" variational formalisms, respectively. Indeed, they are analogous to the metric and Palatini variations used in the study of geometric theories of modified gravity, where in the latter method the metric (Levi-Civita) connection is subsequently imposed in the resulting field equations; the two methods yield the same (second-order) field equations only for the Lovelock action, ${ }^{82-84}$ which coincides with the Einstein-Hilbert action (plus a cosmological constant term) in $D=4$ dimensions.

\section{O. Geometric interpretation of WGT}

So far, we have been firm in our resolve to regard $h_{a}{ }^{\mu}, A^{a b}{ }_{\mu}$, and $B_{\mu}$ purely as gauge fields in Minkowski spacetime and have avoided attaching any geometric interpretation to them. Although we will maintain this viewpoint in this paper, it is nonetheless a common practice to reinterpret WGT in geometric terms, and we therefore give a brief account of this reinterpretation here.

At the heart of the geometric interpretation of WGT (and PGT) is the identification of $h_{a}{ }^{\mu}$ as the components of a vierbein system in a more general spacetime. Thus, at any point $x$ in the spacetime, one demands that the orthonormal tetrad frame vectors $\hat{\boldsymbol{e}}_{a}(x)$ and the coordinate frame 
vectors $\boldsymbol{e}_{\mu}(x)$ are related by ${ }^{85}$

$$
\hat{\boldsymbol{e}}_{a}=h_{a}{ }^{\mu} \boldsymbol{e}_{\mu}, \quad \boldsymbol{e}_{\mu}=b^{a}{ }_{\mu} \hat{\boldsymbol{e}}_{a},
$$

with similar relationships holding between the dual basis vectors $\hat{\boldsymbol{e}}^{a}(x)$ and $\boldsymbol{e}^{\mu}(x)$ in each set. For any other vector $\boldsymbol{J}$, written in the coordinate basis as (say) $J_{\mu} \boldsymbol{e}^{\mu}$, one then identifies the quantities $J_{a}=h_{a}{ }^{\mu} J_{\mu}$, for example, as the components of the same vector, but in the tetrad basis. This is a fundamental difference from the Minkowski spacetime viewpoint presented earlier, in which $\mathcal{J}_{a}=h_{a}{ }^{\mu} J_{\mu}$ would be regarded as the components in the tetrad basis of a new vector field $\mathcal{J}$.

The identification of $h_{a}{ }^{\mu}$ as the components of a vierbein system has a number of far-reaching consequences. First, the index-conversion properties of $h_{a}{ }^{\mu}$ and $b^{a}{ }_{\mu}$ are extended. It is straightforward to show, for example, that $h_{a}{ }^{\mu} J^{a}=J^{\mu}$ and $b^{a}{ }_{\mu} J^{\mu}=J^{a}$. Moreover, any contraction over Latin (Greek) indices can be replaced by one over Greek (Latin) indices. None of these operations is admissible when the $h$ and $A$ fields are viewed purely as gauge fields in Minkowski spacetime.

Perhaps the most important consequence of identifying $h_{a}{ }^{\mu}$ as the components of a vierbein system is that the inner product of the coordinate basis vectors becomes

$$
\boldsymbol{e}_{\mu} \cdot \boldsymbol{e}_{\nu}=\eta_{a b} b^{a}{ }_{\mu} b^{b}{ }_{\nu} \equiv g_{\mu \nu} .
$$

Thus, in this geometric interpretation, one must work in a more general spacetime with metric $g_{\mu \nu}$. Conversely, since the tetrad basis vectors still form an orthonormal set, one has

$$
\hat{\boldsymbol{e}}_{a} \cdot \hat{\boldsymbol{e}}_{b}=\eta_{a b}=g_{\mu \nu} h_{a}{ }^{\mu} h_{b}{ }^{\nu} .
$$

From (115), one also finds that $h^{-1}=\sqrt{-g}$ (where we are working with a metric signature of -2 ). Under a (local, physical) dilation, the spacetime metric and $h$-field have Weyl weights $w\left(g_{\mu \nu}\right)=2$ and $w\left(h_{a}{ }^{\mu}\right)=-1$, respectively, and so (115) and (116) imply that $w\left(\eta_{a b}\right)=0$, as expected. From (114)-(116), one immediately finds that the $h$-field and its inverse are directly related by index raising/lowering, so there is no need to distinguish between them by using different kernel letters. Consequently, the standard practice, which we will follow here, is to notate $h_{a}{ }^{\mu}$ and $b^{a}{ }_{\mu}$ as $e_{a}{ }^{\mu}$ and $e^{a}{ }_{\mu}$, respectively.

One is also led naturally to the interpretation of $A^{a b}{ }_{\mu}$ as the components of the "spinconnection" that encodes the rotation of the local tetrad frame between points $x$ and $x+\delta x$, which is accompanied by a local change in the standard of length between the two points, which is encoded by $B_{\mu}$. Thus, the operation of parallel transport for some vector $J^{a}$ of weight $w$ is defined as

$$
\delta J^{a}=-\left(A_{b \mu}^{a}+w B_{\mu} \delta_{b}^{a}\right) J^{b} \delta x^{\mu},
$$

which is required to compare vectors $J^{a}(x)$ and $J^{a}(x+\delta x)$ at points $x$ and $x+\delta x$, determined with respect to the tetrad frames $\hat{\boldsymbol{e}}_{a}(x)$ and $\hat{\boldsymbol{e}}_{a}(x+\delta x)$, respectively. Hence, in general, a vector not only changes its direction on parallel transport around a closed loop, but also its length. The expression (117) establishes the correct form for the related $(\Lambda, \rho)$-covariant derivative, e.g.,

$$
D_{\mu}^{*} J^{a}=\partial_{\mu} J^{a}+w B_{\mu} J^{a}+A_{b \mu}^{a} J^{b}=\partial_{\mu}^{*} J^{a}+A_{b \mu}^{a} J^{b},
$$

where we have used the partial derivative operator $\partial_{\mu}^{*}$ defined in (25). Moreover, the existence of tetrad frames at each point of the spacetime implies the existence of the Lorentz metric $\eta_{a b}$ at each point. Then demanding that $\eta_{a b}$ is invariant under parallel transport, and recalling that $w\left(\eta_{a b}\right)=0$, requires the spin-connection to be antisymmetric, i.e., $A^{a b}{ }_{\mu}=-A^{b a}{ }_{\mu}$, as previously.

Substantial differences between the Minkowski spacetime gauge field viewpoint and the geometric interpretation do occur, however, when generalising the $(\Lambda, \rho)$-covariant derivative to apply to fields with definite GCT tensor behaviour. First, in the geometric interpretation, one can in general no longer construct a global inertial Cartesian coordinate system in the more general spacetime. Thus, one must rely on arbitrary coordinates and so define the "total" covariant derivative

$$
\Delta_{\mu}^{*} \equiv \partial_{\mu}^{*}+\Gamma_{\rho \mu}^{\sigma} \mathrm{X}_{\sigma}^{\rho}+\frac{1}{2} A^{a b}{ }_{\mu} \Sigma_{a b}=\nabla_{\mu}^{*}+D_{\mu}^{*}-\partial_{\mu}^{*},
$$

where $\nabla_{\mu}^{*}=\partial_{\mu}^{*}+\Gamma_{\rho \mu}^{\sigma} \mathrm{X}^{\rho}{ }_{\sigma}$ and $\mathrm{X}^{\rho}{ }_{\sigma}$ are the GL $(4, R)$ generator matrices appropriate to the GCT tensor character of the field to which $\Delta_{\mu}^{*}$ is applied. If a field $\psi$ carries only Latin indices, then 
$\nabla_{\mu}^{*} \psi=\partial_{\mu}^{*} \psi$ and so $\Delta_{\mu}^{*} \psi=D_{\mu}^{*} \psi$; conversely, if a field $\psi$ carries only Greek indices, then $D_{\mu}^{*} \psi=\partial_{\mu}^{*} \psi$ and so $\Delta_{\mu}^{*} \psi=\nabla_{\mu}^{*} \psi$. When acting on an object of weight $w$, for all these derivative operators the resulting object also transforms covariantly with the same weight $w$.

Most importantly, in a dynamical spacetime, the affine connection coefficients $\Gamma^{\sigma}{ }_{\rho \mu}$ are themselves dynamical variables, no longer fixed by our choice of coordinate system. They are, however, necessarily related to the spin-connection and dilation vector since, provided $J^{a}$ has weight $w=1$, the tetrad components of a vector with coordinate components $J^{\mu}$ should, when parallel transported from $x$ to $x+\delta x$, be equal to $J^{a}+\delta J^{a}$, i.e.,

$$
J^{a}+\delta J^{a}=\left(J^{\mu}+\delta J^{\mu}\right) e_{\mu}^{a}(x+\delta x) .
$$

In other words, the quantities $A^{a b}{ }_{\mu}$ and $\Gamma_{\rho \mu}^{\sigma}$ represent the same geometrical object in two different frames, and hence there remain 44 gravitational field variables in all. From (120), we obtain the relation

$$
\Delta_{\mu}^{*} e^{a}{ }_{\nu} \equiv \partial_{\mu}^{*} e^{a}{ }_{\nu}-\Gamma^{\sigma}{ }_{\nu \mu} e^{a}{ }_{\sigma}+A^{a}{ }_{b \mu} e^{a}{ }_{\nu}=0,
$$

which relates $A$ and $\Gamma$ (and $B$ ); in particular, we note that $w\left(\Gamma^{\sigma}{ }_{v \mu}\right)=0$. The relation (121) is sometimes known as the "tetrad postulate," but note that it always holds. It is straightforward to show that $A$ or $\Gamma$ may be written explicitly in terms of the other as

$$
\begin{aligned}
& \Gamma^{\lambda \mu}=e_{a}{ }^{\lambda}\left(\partial_{\mu}^{*} e^{a}{ }_{\nu}+A^{a}{ }_{b \mu} e^{b}{ }_{v}\right), \\
& A^{a}{ }_{b \mu}=e^{a}{ }_{\lambda}\left(\partial_{\mu}^{*} e_{b}{ }^{\lambda}+\Gamma^{\lambda}{ }_{\nu \mu} e_{b}{ }^{\nu}\right) .
\end{aligned}
$$

Using (115) and (121), one finds that $\nabla_{\sigma}^{*} g_{\mu \nu}=0$, and so this derivative operator commutes with raising and lowering of coordinate indices. Equivalently, one may write this semi-metricity condition as

$$
\nabla_{\sigma} g_{\mu \nu}=-2 B_{\sigma} g_{\mu \nu},
$$

which shows that the spacetime has, in general, a Weyl-Cartan $Y_{4}$ geometry. Hence, in general, the connection is neither metric compatible nor torsion-free. Moreover, substituting (123) into the expressions (39) and (42) for the gauge field strengths $R^{a b}{ }_{\mu \nu}$ and $T^{* a}{ }_{\mu \nu}$, one finds that

$$
\begin{aligned}
R_{\sigma \mu \nu}^{\rho} & =2\left(\partial_{[\mu} \Gamma_{|\sigma| v]}^{\rho}+\Gamma_{\lambda[\mu}^{\rho} \Gamma_{|\sigma| v]}^{\lambda}\right)-H_{\mu \nu} \delta_{\sigma}^{\rho}, \\
T_{\mu \nu}^{* \lambda} & =2 \Gamma_{[v \mu]}^{\lambda}, \\
H_{\mu \nu} & =2 \partial_{[\mu} B_{\nu]},
\end{aligned}
$$

where $R_{\sigma \mu \nu}^{\rho}=e_{a}{ }^{\rho} e^{b}{ }_{\sigma} R_{b \mu \nu}^{a}$ and $T_{\mu \nu}^{* \lambda}=e_{a}^{\lambda} T^{* a}{ }_{\mu \nu}$. Thus, although we recognise $T^{* \lambda}{ }_{\mu \nu}$ as (minus) the torsion tensor of the $Y_{4}$ spacetime, we see that $R_{\sigma \mu \nu}^{\rho}$ is not simply its Riemann tensor. Rather, the Riemann tensor of the $Y_{4}$ spacetime is given by

$$
\widetilde{R}_{\sigma \mu \nu}^{\rho} \equiv R_{\sigma \mu \nu}^{\rho}+H_{\mu \nu} \delta_{\sigma}^{\rho}
$$

One should note that, although $\widetilde{R}_{\rho \sigma \mu \nu}$ is antisymmetric in $(\mu, v)$, it is no longer antisymmetric in $(\rho, \sigma)$ (indeed $\left.\widetilde{R}_{(\rho \sigma) \mu \nu}=g_{\rho \sigma} H_{\mu \nu}\right)$ and does not satisfy the familiar cyclic and Bianchi identities of the Riemann tensor in a Riemannian $V_{4}$ spacetime. One may also show that, with the given arrangements of indices, both $\widetilde{R}_{\sigma \mu \nu}^{\rho}$ (or $R_{\sigma \mu \nu}^{\rho}$ ) and $T^{* \lambda}{ }_{\mu \nu}$ transform covariantly with weight $w=0$ under a local dilation. It is also worth noting that $\widetilde{R}_{\mu \nu} \equiv \widetilde{R}_{\mu \lambda \nu}{ }^{\lambda}=R_{\mu \nu}-H_{\mu \nu}$ and $\widetilde{R} \equiv \widetilde{R}_{\mu}^{\mu}=R$. As one might expect, the quantities (125)-(128) arise naturally in the expression for the commutator of two derivative operators acting on a vector $J^{\rho}$ (say) of Weyl weight $w$, which is given by

$$
\left[\nabla_{\mu}^{*}, \nabla_{\nu}^{*}\right] J^{\rho}=\widetilde{R}_{\sigma \mu \nu}^{\rho} J^{\sigma}+w H_{\mu \nu} J^{\rho}-T_{\mu \nu}^{* \sigma} \nabla_{\sigma}^{*} V^{\rho} .
$$

Finally, from (124), one also finds that the affine connection must satisfy

$$
\Gamma_{\mu \nu}^{\lambda}={ }^{0} \Gamma_{\mu \nu}^{* \lambda}+K_{\mu \nu}^{* \lambda},
$$


where the first term on the RHS reads

$$
\begin{aligned}
{ }^{0} \Gamma_{\mu \nu}^{* \lambda} & =\frac{1}{2} g^{\lambda \rho}\left(\partial_{\mu}^{*} g_{\nu \rho}+\partial_{\nu}^{*} g_{\mu \rho}-\partial_{\rho}^{*} g_{\mu \nu}\right) \\
& ={ }^{0} \Gamma_{\mu \nu}^{\lambda}+\delta_{\nu}^{\lambda} B_{\mu}+\delta_{\mu}^{\lambda} B_{v}-g_{\mu \nu} B^{\lambda},
\end{aligned}
$$

in which ${ }^{0} \Gamma^{\lambda}{ }_{\mu \nu} \equiv \frac{1}{2} g^{\lambda \rho}\left(\partial_{\mu} g_{\nu \rho}+\partial_{\nu} g_{\mu \rho}-\partial_{\rho} g_{\mu \nu}\right)$ is the standard metric (Christoffel) connection and $K^{* \lambda}{ }_{\mu \nu}$ is the $Y_{4}$ contortion tensor

$$
K_{\mu \nu}^{* \lambda}=-\frac{1}{2}\left(T_{\mu \nu}^{* \lambda}-T^{*}{ }^{\lambda}{ }_{\mu}+T^{*}{ }_{\mu \nu}^{\lambda}\right),
$$

for which it is worth noting that $K_{\lambda \mu \nu}^{*}=-K_{\mu \lambda v}^{*}$.

The result (130) is the analogue of the expression (47) in the gauge theory viewpoint. Indeed, it is also of interest to consider briefly the geometric interpretation of the quantities ${ }^{0} A^{* a b}{ }_{\mu}$, introduced in (47), which depend only on the $h$-field, its first derivatives, and the $B$-field. Following an analogous argument to that given above, but considering instead the reduced covariant derivative ${ }^{0} D_{\mu}^{*}$, as defined in (48), one finds that ${ }^{0} A^{* a b}{ }_{\mu}$ and the connection ${ }^{0} \Gamma^{* \sigma}{ }_{\rho \mu}$ represent the same geometrical object in two different frames, and one obtains a "reduced" form of the tetrad postulate (121) given by

$$
{ }^{0} \Delta_{\mu}^{*} e^{a}{ }_{\nu} \equiv \partial_{\mu}^{*} e^{a}{ }_{\nu}-{ }^{0} \Gamma^{* \sigma}{ }_{\nu \mu} e^{a}{ }_{\sigma}+{ }^{0} A^{* a}{ }_{b \mu} e^{b}{ }_{\nu}=0 .
$$

It thus follows that the relationships (122) and (123) again hold with the replacements $\Gamma^{\sigma}{ }_{\nu \mu} \rightarrow$ ${ }^{0} \Gamma^{* \sigma}{ }_{\nu \mu}$ and $A_{b \mu}^{a} \rightarrow{ }^{0} A^{* a}{ }_{b \mu}$, from which one can directly derive (131). One also obtains the metricity condition ${ }^{0} \nabla_{\sigma}^{*} g_{\mu \nu}=0$. Finally, the expression (125) for the curvature also holds, but with $R_{\sigma \mu \nu}^{\rho} \rightarrow{ }^{0} R^{*} \rho_{\sigma \mu \nu}$ and $\Gamma^{\sigma}{ }_{v \mu} \rightarrow{ }^{0} \Gamma^{* \sigma}{ }_{\nu \mu}$, whereas (126) becomes simply ${ }^{0} T^{* \lambda}{ }_{\mu \nu}=0$, indicating the absence of torsion, as expected. The expression (129) is also valid, but with $\nabla_{\mu}^{*} \rightarrow{ }^{0} \nabla_{\mu}^{*}$, $R_{\sigma \mu \nu}^{\rho} \rightarrow{ }^{0} R^{* \rho} \rho_{\sigma \nu}$, and $T^{* \sigma}{ }_{\mu \nu} \rightarrow{ }^{0} T^{* \sigma}{ }_{\mu \nu}=0$.

Finally, it is worth noting that, in any given gravitational theory, one can choose to interpret some quantities as geometric properties of the underlying spacetime and others as fields residing in that spacetime. Indeed, one can place the dividing line anywhere, with the gauge theory approach advocated in this paper and the geometric interpretation just described representing extreme ends of this range of possibilities: the former interprets all quantities as fields residing in a background Minkowski spacetime, whereas the latter interprets all gravitational quantities in terms of geometric properties of the underlying spacetime. This is discussed further in Appendix B, in the context of PGT.

\section{EXTENDED WEYL GAUGE THEORY}

We now consider a novel alternative to standard Weyl gauge theory, which has a number of interesting features. Recall that, in Sections II J and II K, respectively, we showed that the PGT matter actions for the (massless) Dirac field and the electromagnetic field are already invariant under local dilations (and are, indeed, identical to their WGT counterparts), provided the gravitational gauge fields $h_{a}{ }^{\mu}$ and $A^{a b}{ }_{\mu}$ transform under local Weyl transformations as (27) and (23), respectively. Considering just the local dilation part of the transformation, ${ }^{86}$ for the fields $h_{a}{ }^{\mu}$ and $A^{a b}{ }_{\mu}$, one has

$$
\begin{aligned}
h_{a}^{\prime \mu}(x) & =e^{-\rho(x)} h_{a}{ }^{\mu}(x), \\
A^{\prime a b}{ }_{\mu}(x) & =A^{a b}{ }_{\mu}(x),
\end{aligned}
$$

showing that they transform covariantly with weights -1 and 0 , respectively (where we have temporarily reinstated the explicit notational dependence of the fields on spacetime position $x$ ).

It is straightforward to show, however, that the Dirac and electromagnetic field actions in PGT (and WGT) remain invariant under local dilations, even if one assumes a more general "extended" transformation law for the $A$-field, whilst still retaining the original $h$-field transformation law, such 
that (134) and (135) are replaced by

$$
\begin{aligned}
h_{a}^{\prime \mu} & =e^{-\rho} h_{a}{ }^{\mu}, \\
A^{\prime a b}{ }_{\mu} & =A^{a b}{ }_{\mu}+\theta\left(b^{a}{ }_{\mu} \mathcal{P}^{b}-b^{b}{ }_{\mu} \mathcal{P}^{a}\right),
\end{aligned}
$$

where $P_{v} \equiv \partial_{v} \rho, \mathcal{P}_{a} \equiv h_{a}{ }^{v} P_{v}$, and $\theta$ is an arbitrary parameter that can take any value. ${ }^{87}$ Moreover, under a global scale transformation, the "extended" transformation laws (136) and (137) for the $h$ and $A$ fields reduce to the same form as the "normal" ones (134) and (135) and may be considered as an equally valid gauging of global scale invariance. These observations provide compelling motivation for exploring the properties of the gauge theory of local Weyl transformations in which the $h$ and $A$ fields obey the transformations (136) and (137) under local dilations; we call this extended Weyl gauge theory (eWGT).

A complementary motivation for exploring eWGT is that the "extended" transformation laws (136) and (137) lead to transformation properties for the PGT curvature $\mathcal{R}^{a b}{ }_{c d}$ and torsion $\mathcal{T}^{a}{ }_{b c}$ that are on a more equal footing with one another than those that result from the standard WGT transformations laws (134) and (135). Under the latter set of transformations, $\mathcal{R}^{a b}{ }_{c d}$ and $\mathcal{T}^{a}{ }_{b c}$ behave in very different ways. The PGT curvature $\mathcal{R}^{a b}{ }_{c d}$ transforms covariantly with weight $w=-2$ and also acts as the field strength tensor of the $A$ gauge field in WGT. By contrast, the PGT torsion $\mathcal{T}^{a}{ }_{b c}$ transforms inhomogeneously as

$$
\mathcal{T}^{\prime a}{ }_{b c}=e^{-\rho}\left(\mathcal{T}^{a}{ }_{b c}+\mathcal{P}_{b} \delta_{c}^{a}-\mathcal{P}_{c} \delta_{b}^{a}\right),
$$

and one must instead introduce $\mathcal{T}^{* a} b c$ as the field strength tensor for the $h$ gauge field in WGT. Indeed, the transformations (134) and (135) have the consequence of inducing PGT torsion. Considering the case where the $h$-field originally has vanishing PGT torsion $\mathcal{T}^{a}{ }_{b c}=0$, the inhomogeneous nature of the transformation law (138) means that the local dilation leads to $\mathcal{T}^{\prime a}{ }_{b c} \neq 0$.

Assuming the "extended" transformation laws (136) and (137), however, the PGT curvature and torsion transform under local dilations as

$$
\begin{aligned}
\mathcal{R}^{\prime a b}{ }_{c d}= & e^{-2 \rho}\left\{\mathcal{R}^{a b}{ }_{c d}+2 \theta \delta_{d}^{[a}\left(\mathcal{D}_{c}-\theta \mathcal{P}_{c}\right) \mathcal{P}^{b]}-2 \theta \delta_{c}^{[a}\left(\mathcal{D}_{d}-\theta \mathcal{P}_{d}\right) \mathcal{P}^{b]}\right. \\
& \left.-2 \theta \mathcal{P}^{[a} \mathcal{T}^{b]}{ }_{c d}-2 \theta^{2} \delta_{c}^{[a} \delta_{d}^{b]} \mathcal{P}^{e} \mathcal{P}_{e}\right\}, \\
\mathcal{T}^{\prime a}{ }_{b c}= & e^{-\rho}\left\{\mathcal{T}^{a}{ }_{b c}+2(1-\theta) \mathcal{P}_{[b} \delta_{c]}^{a}\right\} .
\end{aligned}
$$

Thus, for general values of $\theta$, neither $\mathcal{R}^{a b}{ }_{c d}$ nor $\mathcal{T}^{\dagger a}{ }_{b c}$ transforms covariantly, but for $\theta=0$ one recovers the "normal" transformation law (135) for the $A$-field, which results in a covariant transformation law for the PGT curvature under local dilations, whereas for $\theta=1$ one obtains the "special" transformation law for the $A$-field,

$$
A^{\prime a b}{ }_{\mu}=A^{a b}{ }_{\mu}+\left(b^{a}{ }_{\mu} \mathcal{P}^{b}-b^{b}{ }_{\mu} \mathcal{P}^{a}\right),
$$

which leads to a covariant transformation law with weight $w=-1$ for the PGT torsion. By considering the "extended" A-field transformation law (137), one can therefore accommodate both cases in a balanced manner. The corresponding transformation laws for the contractions of the PGT curvature and torsion are given explicitly in Section V A, and those for the "reduced" PGT curvature ${ }^{0} \mathcal{R}^{a b}{ }_{c d}$ and its contractions (see Appendix B) are given in Section V B; the "reduced" PGT torsion ${ }^{0} \mathcal{T}{ }^{a} b c$ vanishes identically.

\section{A. Extended local Weyl invariance}

As usual, our first task is to define a method for converting a matter action invariant under global Weyl transformations into one that is invariant under local Weyl transformations, but where the $h$ and $A$ fields are now assumed to transform under local dilations as (136) and (137), respectively. From now on we will call this combination "extended local Weyl transformations," although it should be noted that we are not extending the form of GCT, local Lorentz, or local scale transformations under consideration, but merely assuming an alternative (or "extended") form for the transformation of the $A$-field under local dilations. In particular, we require the action to be invariant 
for arbitrary values of the parameter $\theta$ in (137). Following the standard gauge-theory approach, we construct a new covariant derivative that transforms under these transformations in the same way as the standard partial derivative transforms under global Weyl transformations, namely, according to (8). As in WGT, we construct the covariant derivative in two stages.

In the first step, we construct a $(\Lambda, \rho)$-covariant derivative $D_{\mu}^{\dagger} \varphi$ by introducing a dilation vector gauge field (called $V_{\mu}$ to distinguish it from the dilation gauge field $B_{\mu}$ in WGT). In constructing this derivative, the $A$-field transformation law (137) suggests the introduction of a modified $A$-field of the form

$$
A_{\mu}^{\dagger a b} \equiv A_{\mu}^{a b}+\left(\mathcal{V}^{a} b^{b}{ }_{\mu}-\mathcal{V}^{b} b^{a}{ }_{\mu}\right),
$$

where $\mathcal{V}_{a}=h_{a}{ }^{\mu} V_{\mu}$. Clearly, by construction, $A^{\dagger a b}{ }_{\mu}$ is also antisymmetric in $a$ and $b$. It is worth noting that we do not consider $A^{\dagger a b}{ }_{\mu}$ to be a fundamental field, but merely a shorthand for the combination of the gauge fields $h_{a}{ }^{\mu}$ (or its inverse), $A^{a b}{ }_{\mu}$, and $V_{\mu}$ on the RHS of (142). As in WGT, one must also include terms in the covariant derivative to cancel terms arising from $\partial_{\mu} \varphi^{\prime}$ and, in this case, also from the transformation of $A^{\dagger a b}{ }_{\mu}$. By demanding that $D_{\mu}^{\dagger} \varphi$ transforms in the same way as (22) under extended local Weyl transformations, one finds that the appropriate form for the covariant derivative is ${ }^{88}$

$$
D_{\mu}^{\dagger} \varphi \equiv\left(\partial_{\mu}+\frac{1}{2} A_{\mu}^{\dagger a b} \Sigma_{a b}-w V_{\mu}-\frac{1}{3} w T_{\mu}\right) \varphi,
$$

in which $w$ is the Weyl weight of the field $\varphi$ and $T_{\mu}=b^{a}{ }_{\mu} \mathcal{T}_{a}$, where $\mathcal{T}_{a}$ is the trace of the PGT torsion (see Appendix B), obtained by removing the asterisks from Equation (66). Here we consider $T_{\mu}$ merely as a shorthand for this corresponding function of the gauge fields $h_{a}{ }^{\mu}$ and $A^{a b}{ }_{\mu}$. Under the extended local scale transformations (136)-(137), it is straightforward to show that $T_{\mu}^{\prime}=T_{\mu}+3(1-\theta) P_{\mu}$ and so (143) does indeed transform covariantly with Weyl weight $w$ provided that the dilation gauge field transforms (inhomogeneously, as expected) according to

$$
V_{\mu}^{\prime}=V_{\mu}+\theta P_{\mu},
$$

which is the "extended" counterpart to (24) in WGT. This transformation law also ensures that the modified $A$-field (142) is invariant under the extended scale transformations (136)-(137), i.e., $A^{\dagger \prime a b}{ }_{\mu}=A^{\dagger a b}{ }_{\mu}$ and so it has a weight $w=0$. Note how this emulates the invariance property (135) of the original $A$-field under normal scale transformations.

An alternative approach to obtaining the form of the covariant derivative (143) and the $V$-field transformation law (144) is given in Appendix E, where we demonstrate that the Weyl weight $w$ of the field $\varphi$ on which the covariant derivative acts does not need to be inserted into (143) "by hand," but instead arises naturally, in contrast to standard WGT, and (143) is picked out as the unique form for the eWGT covariant derivative.

It is important to note that the covariant derivative (143) does not explicitly contain the parameter $\theta$. Consequently, it does not reduce to the standard WGT covariant derivative $D_{\mu}^{*} \varphi$ (with $B_{\mu}$ replaced by $V_{\mu}$ ) in the case $\theta=0$, in which one recovers the "normal" local Weyl transformations. Indeed, in this case, one sees from (144) that one can covariantly set $V_{\mu}=0$, so that $A^{\dagger a b}{ }_{\mu}=A^{a b}{ }_{\mu}$ and the covariant derivative (143) may be written simply as $\left.D_{\mu}^{\dagger} \varphi\right|_{\theta=0}=\left(D_{\mu}-\frac{1}{3} w T_{\mu}\right) \varphi$, in which the role played by the usual dilation gauge field $B_{\mu}$ in WGT has been taken up instead by the trace of the PGT torsion; such a replacement has been discussed previously. ${ }^{89}$ Conversely, in the case $\theta=1$, for which one recovers the "special" transformation (141) for the $A$-field, one can covariantly set the PGT torsion to zero, and hence $T_{\mu}=0$, so that the covariant derivative (143) may be written as $\left.D_{\mu}^{\dagger} \varphi\right|_{\theta=1} \equiv\left(\partial_{\mu}+\frac{1}{2} A^{\dagger a b} \Sigma_{a b}-w V_{\mu}\right) \varphi$.

For the remainder of our discussion, however, we will consider the general case in which the parameter $\theta$ may take any value. As for any gauge theory, the corresponding covariant derivative (143) defines the structure of eWGT. Indeed, in principle, one could forgo the preceding discussion and simply start with the definition (143), together with (142) and the transformation laws of $h_{a}{ }^{\mu}$, $A^{a b}{ }_{\mu}$, and $V_{\mu}$, to construct eWGT. By analogy with WGT, we note that it is sometimes convenient to write the $(\Lambda, \rho)$-covariant derivative (143) as

$$
D_{\mu}^{\dagger} \varphi=\left(\partial_{\mu}^{\dagger}+\frac{1}{2} A_{\mu}^{\dagger a b} \Sigma_{a b}\right) \varphi
$$


where we have defined the derivative operator

$$
\partial_{\mu}^{\dagger} \equiv \partial_{\mu}-w\left(V_{\mu}+\frac{1}{3} T_{\mu}\right) .
$$

It is straightforward to show that $\partial_{\mu}^{\dagger} \varphi(x)$ itself also transforms covariantly with weight $w$ (i.e., the same weight as the field $\varphi$ ) under local dilations assuming the extended transformation laws (136) and (137) (but does not transform covariantly under local Poincaré transformations).

In the second step of the gauging process, precisely as in WGT, we define a generalised covariant derivative

$$
\mathcal{D}_{a}^{\dagger} \varphi \equiv h_{a}{ }^{\mu} D_{\mu}^{\dagger} \varphi
$$

which transforms covariantly with weight $w-1$, as desired. Having achieved our aim of constructing an appropriate covariant derivative, we can now straightforwardly convert a matter action invariant under global Weyl transformations into one that is invariant under extended local Weyl transformations, which is given by

$$
S_{\mathrm{M}}=\int h^{-1} L_{\mathrm{M}}\left(\varphi, \mathcal{D}_{a}^{\dagger} \varphi\right) d^{4} x
$$

Once again, it is convenient to denote the integrand of the action by the Lagrangian density $\mathcal{L}_{\mathrm{M}} \equiv h^{-1} L_{\mathrm{M}}\left(\varphi, \mathcal{D}^{\dagger} \varphi\right)$. We note that the gravitational gauge fields $h, A$, and $V$ in eWGT contain a total of 44 independent variables, as in WGT. As was the case in WGT, (148) is not guaranteed to inherit invariance properties possessed by original action under other types of transformation, so (148) may need to be modified to satisfy any further required invariances.

As in WGT, one may also introduce an additional "compensator" scalar field $\phi$ (or fields) into the matter action, which opens up possibilities for the inclusion of further terms in the matter action that non-minimally (conformally) couple $\phi$ to the eWGT gravitational gauge field strengths (see Section III D). In particular, terms proportional to $\phi^{2} \mathcal{R}^{\dagger}$ or $\phi^{2} L_{\mathcal{T}^{\dagger 2}}$ are extended Weyl-covariant with weight $w=-4$ and so may be added to $L_{\mathrm{M}}$.

\section{B. Relationship to other approaches}

Before continuing with the formal development of eWGT, it is worth commenting briefly on the relationship of our approach to others in the literature. In particular, by introducing the modified $A$-field in (142), one might ask whether the modified transformation law in (137) corresponds to some "deformation" of the action of the Weyl group, which underlies the physical symmetries we are assuming. Indeed, it might seem that such a deformation does occur if one takes the view that specifying the group action leads uniquely to a given set of gauge fields and transformation laws; in some approaches these do follow directly from the structure constants of the associated Lie group.

There have, for example, been several attempts in the literature to carry out local gauging not just for the Weyl group, but for the full conformal group, i.e., with special conformal translations included as well as rotations, translations, and dilations. An early approach to this by Kaku et al. ${ }^{90}$ considers the representation of the full conformal group $\mathrm{O}(4,2)$ via the $4 \times 4$ Dirac matrix algebra of $\mathrm{SU}(2,2)$, to which $\mathrm{O}(4,2)$ is locally isomorphic. In this approach, the structure constants of the group to be locally gauged feed through via the Maurer-Cartan equations to give the "curvatures" corresponding to the different group elements. These equations are therefore fixed by the initial group structure.

Of course, such a method is very different to that which we carry out here. We emphasise in particular that after the decoupling of the translation and dilational parts of the transformation described at the start of Section II B, the form of scaling we describe here corresponds to "Weyl scaling," as discussed, for example, in Section 2.2 of Blagojevic. ${ }^{10}$ In this case, all fields are rescaled according to their Weyl weight, but without any accompanying coordinate changes. This is still exactly the symmetry for which we are providing a local gauging via our modified $A$-field (142), and modified transformation laws (137), and thus no "deformation" has taken place of the underlying symmetry. In particular, the associated group is still "Weyl scaling," despite the changes in the implementation of it. 
It would, however, still be of interest to attempt to relate the proposed new transformations and extended $A$-field found here with attempts at full conformal gauging, such as carried out by Kaku et al., since some intriguing relationships between the two approaches do exist. In particular, both Kaku et al. and later Wheeler, ${ }^{91}$ find that the gauge field corresponding to special conformal transformations, which in their approaches is an additional "vierbein-like" field similar to the field corresponding to translations, does not propagate and can be eliminated via its equations of motion. This leaves a version of "Weyl scaling" as the only further symmetry remaining in addition to translational and rotational symmetries.

The interest for our approach lies in the fact that, during the gauging process, additional components of the spin connection are found that are of similar form to the new piece of the $A$-field we are proposing. An explicit example of this can be seen in equation (6) of Kaku et al., in which a piece $e_{b \mu} b_{a}-e_{a \mu} b_{b}$ is added to the spin connection $\omega_{\mu a b}$ (their $b$-field is the "dilatation field," which in our case corresponds to $\mathcal{V}$, while their $e_{a \mu}$ is a version of either our $h$-field or its inverse; it is difficult to make an exact correspondence due to the very different way the translation gauge field is treated between their approach and ours).

Furthermore, one of the problems of gauging the conformal group, which the approach of Kaku et al. is trying to overcome, lies in the fact that the global group generators in a coordinate representation involve a naked $4 \mathrm{D}$ position vector, $x^{\mu}$, and a local equivalent of this does not exist. However, if we nevertheless examine global conformal transformations, then Section 4.1 of Blagojevic ${ }^{10}$ shows that how variation under these leads to a modified Lorentz spin connection containing an extra term of the form $c_{b \mu} x_{a}-c_{a \mu} x_{b}$, where here $c_{a \mu}$ is the extra "vierbein-type" field corresponding to the special conformal transformations. Here $x_{a}$ is a global surrogate for $\mathcal{V}_{a}$ (noting the fact that the generator of global dilations is $x \cdot \nabla$ ), and so this is again suggestive that our proposed additional term in the Lorentz spin connection given in (142), and the modified transformation laws (137) (involving an arbitrary new parameter $\theta$, which in the end does not appear in the covariant derivative itself), may be linked with the symmetries of the full conformal group and may even provide a new route through to its successful gauging. We leave this topic to future research, however, and now continue with our development of eWGT.

\section{Minkowski spacetime interpretation of eWGT}

The comments made in Section II C also hold in eWGT, in particular the freedom to choose global inertial Cartesian coordinates in the Minkowski spacetime and, if required, the way in which the covariant derivative can be extended to act on quantities with a definite tensor behaviour under GCT. In particular, the $(\Lambda, \rho)$-covariant derivative in (143) becomes

$$
D_{\mu}^{\dagger}=\partial_{\mu}^{\dagger}+{ }^{0} \Gamma_{\rho \mu}^{\sigma} \mathrm{X}_{\sigma}^{\rho}+\frac{1}{2} A^{\dagger a b}{ }_{\mu} \Sigma_{a b}={ }^{0} \nabla_{\mu}^{\dagger}+\frac{1}{2} A^{\dagger a b}{ }_{\mu} \Sigma_{a b},
$$

where ${ }^{0} \nabla_{\mu}^{\dagger} \equiv{ }^{0} \nabla_{\mu}-w\left(V_{\mu}+\frac{1}{3} T_{\mu}\right),{ }^{0} \Gamma_{\mu \nu}^{\lambda} \equiv \frac{1}{2} \gamma^{\lambda \rho}\left(\partial_{\mu} \gamma_{\nu \rho}+\partial_{\nu} \gamma_{\mu \rho}-\partial_{\rho} \gamma_{\mu \nu}\right)$ is the metric connection corresponding to the (flat-space) metric $\gamma_{\mu \nu}$ defined by the coordinate system, and $\mathrm{X}^{\rho}{ }_{\sigma}$ are the $\mathrm{GL}(4, R)$ generator matrices appropriate to the GCT tensor character of the field to which $D_{\mu}^{\dagger}$ is applied, and $w$ is its Weyl weight.

\section{Gauge field strengths in eWGT}

As in WGT, we define the gauge field strengths in terms of the commutator of covariant derivatives. Considering first the $\operatorname{eWGT}(\Lambda, \rho)$-covariant derivative, one finds that

$$
\left[D_{\mu}^{\dagger}, D_{\nu}^{\dagger}\right] \varphi=\frac{1}{2} R_{\mu \nu}^{\dagger a b} \Sigma_{a b} \varphi-w H_{\mu \nu}^{\dagger} \varphi,
$$

which is clearly of a similar form to the corresponding result (38) in WGT, but the eWGT field strengths have very different forms to their WGT counterparts, in particular their dependencies on the gauge fields. 
The eWGT rotational field strength tensor is found to have the form

$$
\begin{aligned}
R_{\mu \nu}^{\dagger a b} & \equiv \partial_{\mu} A^{\dagger a b}{ }_{\nu}-\partial_{\nu} A^{\dagger a b}{ }_{\mu}+A^{\dagger a}{ }_{c \mu} A^{\dagger c b}{ }_{\nu}-A^{\dagger a}{ }_{c \nu} A^{\dagger c b}{ }_{\mu} \\
& =R^{a b}{ }_{\mu \nu}+4 b^{[b}{ }_{[\nu} D_{\mu]} \mathcal{V}^{a]}+4 \mathcal{V}^{[a} V_{[\mu} b^{b]}{ }_{\nu]}-2 \mathcal{V}^{e} \mathcal{V}_{e} b^{[a}{ }_{\mu} b^{b]}{ }_{\nu}+2 \mathcal{V}^{[a} T^{b]}{ }_{\mu \nu},
\end{aligned}
$$

where $R^{a b}{ }_{\mu \nu}$ is the rotational gauge field strength (curvature) in PGT (and WGT), $T^{a}{ }_{\mu \nu}$ is the PGT translational gauge field (torsion), and $D_{\mu}$ is the PGT covariant derivative. The eWGT dilation gauge field strength reads

$$
H_{\mu \nu}^{\dagger}=\partial_{\mu}\left(V_{\nu}+\frac{1}{3} T_{\nu}\right)-\partial_{\nu}\left(V_{\mu}+\frac{1}{3} T_{\mu}\right) .
$$

As in WGT, both $R^{\dagger a b}{ }_{\mu \nu}$ and $H_{\mu \nu}^{\dagger}$ transform covariantly under GCT and local Lorentz rotations in accordance with their respective index structures and are invariant under extended local dilations.

Considering instead the commutator of two generalised covariant derivatives, one finds

$$
\left[\mathcal{D}_{c}^{\dagger}, \mathcal{D}_{d}^{\dagger}\right] \varphi=\frac{1}{2} \mathcal{R}^{\dagger a b}{ }_{c d} \Sigma_{a b} \varphi-w \mathcal{H}_{c d}^{\dagger} \varphi-\mathcal{T}^{\dagger a}{ }_{c d} \mathcal{D}_{a}^{\dagger} \varphi
$$

where $\mathcal{R}^{\dagger a b}{ }_{c d}=h_{c}{ }^{\mu} h_{d}{ }^{\nu} R^{\dagger a b}{ }_{\mu \nu}$ and $\mathcal{H}_{c d}^{\dagger}=h_{c}{ }^{\mu} h_{d}{ }^{\nu} H_{\mu \nu}^{\dagger}$, and the translational field strength is now given by

$$
\mathcal{T}^{\dagger a}{ }_{b c} \equiv h_{b}{ }^{\mu} h_{c}{ }^{\nu}\left(D_{\mu}^{\dagger} b^{a}{ }_{\nu}-D_{\nu}^{\dagger} b^{a}{ }_{\mu}\right) \equiv h_{b}{ }^{\mu} h_{c}{ }^{\nu} T^{\dagger a}{ }_{\mu \nu} .
$$

It is easy to show that, similarly to WGT, $\mathcal{R}^{\dagger a b}{ }_{c d}, \mathcal{H}_{c d}^{\dagger}$, and $\mathcal{T}^{\dagger a}{ }_{c d}$ transform covariantly under extended local dilations with weights $w\left(\mathcal{R}^{\dagger a b}{ }_{c d}\right)=w\left(\mathcal{H}_{c d}^{\dagger}\right)=-2$ and $w\left(\mathcal{T}^{\dagger a}{ }_{c d}\right)=-1$, respectively.

For later convenience, it is worth noting that the explicit forms of $\mathcal{R}^{\dagger a b}{ }_{c d}$ and its contractions are given in terms of their PGT (and WGT) counterparts by

$$
\begin{aligned}
\mathcal{R}^{\dagger a b}{ }_{c d} & =\mathcal{R}^{a b}{ }_{c d}+2 \delta_{d}^{[b}\left(\mathcal{D}_{c}+\mathcal{V}_{c}\right) \mathcal{V}^{a]}-2 \delta_{c}^{[b}\left(\mathcal{D}_{d}+\mathcal{V}_{d}\right) \mathcal{V}^{a]}-2 \mathcal{V}^{e} \mathcal{V}_{e} \delta_{c}^{[a} \delta_{d}^{b]}+2 \mathcal{V}^{[a} \mathcal{T}^{b]}{ }_{c d}, \\
\mathcal{R}^{\dagger a}{ }_{c} & =\mathcal{R}^{a}{ }_{c}+2\left(\mathcal{D}_{c}+\frac{1}{2} \mathcal{T}_{c}+\mathcal{V}_{c}\right) \mathcal{V}^{a}+\delta_{c}^{a}\left(\mathcal{D}_{b}-2 \mathcal{V}_{b}\right) \mathcal{V}^{b}-\mathcal{T}^{a}{ }_{c b} \mathcal{V}^{b}, \\
\mathcal{R}^{\dagger} & =\mathcal{R}+6\left(\mathcal{D}_{a}+\frac{1}{3} \mathcal{T}_{a}-\mathcal{V}_{a}\right) \mathcal{V}^{a},
\end{aligned}
$$

where $\mathcal{D}_{a}$ is the general PGT covariant derivative. Similarly, $\mathcal{T}^{\dagger a}{ }_{b c}$ is given in terms of its PGT counterpart by

$$
\mathcal{T}^{\dagger a}{ }_{b c}=\mathcal{T}^{a}{ }_{b c}+\frac{1}{3}\left(\delta_{b}^{a} \mathcal{T}_{c}-\delta_{c}^{a} \mathcal{T}_{b}\right) .
$$

It is particularly important to note from (156) that the trace of the eWGT torsion vanishes identically, namely,

$$
\mathcal{T}_{b}^{\dagger} \equiv \mathcal{T}^{\dagger a}{ }_{b a}=0
$$

so that $\mathcal{T}^{\dagger a} b c$ is completely trace-free (contraction on any pair of indices yields zero). As we will see below, the property (157) has some desirable and interesting consequences for eWGT. Clearly, the dilational gauge field strength $\mathcal{H}_{a b}^{\dagger}$ is antisymmetric in $a$ and $b$ and hence also has no non-trivial contractions.

The functional dependencies of the three field strengths tensors on the gauge fields and their derivatives are $\mathcal{R}^{\dagger a b}{ }_{c d}(h, A, \partial A, V, \partial V), \mathcal{T}^{\dagger a}{ }_{b c}(h, \partial h, A)$, and $\mathcal{H}_{a b}^{\dagger}\left(h, \partial h, \partial^{2} h, A, \partial A, \partial V\right)$. It is important to note that these functional dependencies differ markedly from those in standard WGT. In particular, $\mathcal{R}^{\dagger a b}{ }_{c d}$ depends on the dilation gauge field $V$ and it first derivatives, whereas its WGT counterpart has no dependence on the WGT dilation gauge field $B$. By contrast, the translational field strength $\mathcal{T}^{\dagger a}{ }_{b c}$ does not depend on the dilation gauge field, whereas its WGT counterpart does. Finally, the most profound difference occurs for the dilation field strength $\mathcal{H}_{a b}^{\dagger}$, which depends on $\partial h, \partial^{2} h, A$, and $\partial A$, in addition to the dependency, in common with its WGT counterpart, on $h$ and the first derivative of the dilation gauge field. The dependence of $\mathcal{H}_{a b}^{\dagger}$ on the second derivative $\partial^{2} h$ is a particularly unusual feature as compared with typical gauge theories and is discussed in detail in Section IV B. 


\section{E. Alternative form of covariant derivative in eWGT}

In a similar manner to that discussed in Section II E for WGT, one may obtain an alternative form for the covariant derivative in eWGT. By analogy with (47) in WGT, it is straightforward to show that

$$
\mathcal{A}_{a b c}^{\dagger}={ }^{0} \mathcal{A}_{a b c}^{\dagger}(h, \partial h, A, V)+\mathcal{K}_{a b c}^{\dagger}(h, \partial h, A),
$$

in which we have defined the quantities ${ }^{0} \mathcal{A}_{a b c}^{\dagger} \equiv \frac{1}{2}\left(c_{a b c}^{\dagger}+c_{b c a}^{\dagger}-c_{c a b}^{\dagger}\right)$, where $c^{\dagger} c_{a b} \equiv h_{a}{ }^{\mu} h_{b}{ }^{v}$ $\left(\partial_{\mu}^{\dagger} b^{c}{ }_{\nu}-\partial_{\nu}^{\dagger} b^{c}{ }_{\mu}\right)$, and $\mathcal{K}_{a b c}^{\dagger} \equiv-\frac{1}{2}\left(\mathcal{T}_{a b c}^{\dagger}+\mathcal{T}_{b c a}^{\dagger}-\mathcal{T}_{c a b}^{\dagger}\right)$. As before, both ${ }^{0} \mathcal{A}_{a b c}^{\dagger}$ and $\mathcal{K}_{a b c}^{\dagger}$ are antisymmetric in their first two indices. There is a fundamental difference with WGT, however, since ${ }^{0} \mathcal{A}_{a b c}^{\dagger}$ itself depends on the $A$ gauge field, whereas this was not the case previously. Less importantly, $\mathcal{K}_{a b c}^{\dagger}$ is independent of the dilation gauge field, unlike its WGT counterpart. ${ }^{92}$

Under an extended local Weyl transformation, the quantities ${ }^{0} \mathcal{A}_{a b c}^{\dagger}$ transform in the same way as $\mathcal{A}_{a b c}^{\dagger}$, whereas $\mathcal{K}_{a b c}^{\dagger}$ transform as the components of a local tensor with weight $w=-1$. Thus, one can construct the "reduced" $(\Lambda, \rho)$-covariant derivative

$$
{ }^{0} D_{\mu}^{\dagger} \varphi \equiv\left(\partial_{\mu}^{\dagger}+\frac{1}{2}^{0} A^{\dagger a b}{ }_{\mu} \Sigma_{a b}\right) \varphi
$$

which transforms in the same way as $D_{\mu}^{\dagger} \varphi$ under an extended local Weyl transformation. The key difference in eWGT, however, is that ${ }^{0} D_{\mu}^{\dagger} \varphi$ still depends on the $A$ gauge field, and so is less deserving of the description "reduced" than its counterpart in WGT. One can define the corresponding generalised covariant derivative ${ }^{0} \mathcal{D}_{a}^{\dagger} \equiv h_{a}{ }^{\mu 0} D_{\mu}^{\dagger}$, which consequently transforms in the same way as $\mathcal{D}_{a}^{\dagger}$, as required. The "full" generalised covariant derivative is given in terms of the "reduced" one by the alternative form

$$
\mathcal{D}_{a}^{\dagger} \varphi=\left({ }^{0} \mathcal{D}_{a}^{\dagger}+\frac{1}{2} \mathcal{K}^{\dagger b c}{ }_{a} \Sigma_{b c}\right) \varphi
$$

which illustrates that, if one (covariantly) sets the eWGT torsion (and hence contortion) to zero, then $\mathcal{D}_{a}^{\dagger}$ reduces to ${ }^{0} \mathcal{D}_{a}^{\dagger}$.

\section{F. Bianchi identities in eWGT}

One may calculate the Bianchi identities satisfied by the gravitational gauge field strengths $\mathcal{R}^{\dagger a b}{ }_{c d}, \mathcal{T}^{\dagger a}{ }_{b c}$, and $\mathcal{H}_{a b}^{\dagger}$ in eWGT by applying the Jacobi identity to the generalised covariant derivative $\mathcal{D}_{a}^{\dagger}$, in a similar manner to that used in Section II F for WGT.

One quickly finds the three Bianchi identities

$$
\begin{aligned}
\mathcal{D}_{[a}^{\dagger} \mathcal{R}^{\dagger d e}{ }_{b c]}-\mathcal{T}^{\dagger f}{ }_{[a b} \mathcal{R}^{\dagger d e}{ }_{c] f} & =0, \\
\mathcal{D}_{[a}^{\dagger} \mathcal{T}^{\dagger d}{ }_{b c]}-\mathcal{T}^{\dagger e}{ }_{[a b} \mathcal{T}^{\dagger d}{ }_{c] e}-\mathcal{R}_{[a b c]}^{\dagger d}+\mathcal{H}_{[a b}^{\dagger} \delta_{c]}^{d} & =0, \\
\mathcal{D}_{[a}^{\dagger} \mathcal{H}_{b c]}^{\dagger}-\mathcal{T}^{\dagger e}{ }_{[a b} \mathcal{H}^{\dagger}{ }_{c] e} & =0 .
\end{aligned}
$$

Similar to WGT, for the special case in which the "torsion" is totally antisymmetric, such that $\mathcal{T}_{a b c}^{\dagger}=\epsilon_{a b c d} t^{d}$ for some vector $t^{d}$, then one may show that the second term on the LHS of (161b) vanishes.

By contracting over the indices $a$ and $d$ in the "R-identity" (161a), once obtains the oncecontracted Bianchi identity

$$
\mathcal{D}_{a}^{\dagger} \mathcal{R}^{\dagger a e}{ }_{b c}-2 \mathcal{D}_{[b}^{\dagger} \mathcal{R}^{\dagger e}{ }_{c]}-2 \mathcal{T}^{\dagger f}{ }_{a[b} \mathcal{R}^{\dagger a e}{ }_{c] f}-\mathcal{T}^{\dagger f}{ }_{b c} \mathcal{R}^{\dagger e}{ }_{f}=0 .
$$

Contracting again over $b$ and $e$, one then finds the twice-contracted Bianchi identity

$$
\mathcal{D}_{a}^{\dagger}\left(\mathcal{R}^{\dagger a}{ }_{c}-\frac{1}{2} \delta_{c}^{a} \mathcal{R}^{\dagger}\right)+\mathcal{T}^{\dagger f}{ }_{b c} \mathcal{R}^{\dagger b}{ }_{f}+\frac{1}{2} \mathcal{T}^{\dagger f}{ }_{a b} \mathcal{R}^{\dagger a b}{ }_{c f}=0 .
$$

Turning to the " $\mathcal{T}$-identity" (161b) and contracting over $a$ and $d$, one obtains the further once-contracted Bianchi identity

$$
\mathcal{D}_{a}^{\dagger} \mathcal{T}_{b c}^{\dagger a}+2 \mathcal{R}_{[b c]}^{\dagger}+2 \mathcal{H}_{b c}^{\dagger}=0 .
$$


It is worth noting that this is somewhat simpler than the corresponding Bianchi identity (56) in WGT, because of the condition (157) for the automatic vanishing of the eWGT torsion trace. Thus, even in the most general case, the covariant divergence of the translation gauge field strength depends only the rotational and dilational gauge field strengths. It is clear that the " $\mathcal{H}$-identity" (161c) has no non-trivial contractions.

\section{G. Free gravitational action in eWGT}

The construction of the free gravitational action in eWGT is analogous to the approach adopted in WGT and discussed in Section II G. In principle, from $\mathcal{R}_{a b c d}^{\dagger}, \mathcal{T}_{a b c}^{\dagger}$, and $\mathcal{H}_{a b}^{\dagger}$, one can construct a free gravitational action of the general form

$$
S_{\mathrm{G}}=\int h^{-1} L_{\mathrm{G}}\left(\mathcal{R}_{a b c d}^{\dagger}, \mathcal{T}_{a b c}^{\dagger}, \mathcal{H}_{a b}^{\dagger}\right) d^{4} x,
$$

where the requirement of local scale invariance imposes the constraint that $L_{\mathrm{G}}$ must be a relative scalar with Weyl weight $w\left(L_{\mathrm{G}}\right)=-4$. Hence, once again, $L_{\mathrm{G}}$ can be quadratic in $\mathcal{R}_{a b c d}^{\dagger}$ and $\mathcal{H}_{a b}^{\dagger}$, but terms linear in $\mathcal{R}^{\dagger} \equiv \mathcal{R}^{\dagger a b}{ }_{a b}$ or quadratic in $\mathcal{T}_{a b c}^{\dagger}$ are not allowed. Similarly, higher-order terms in $\mathcal{R}_{a b c d}^{\dagger}$ and $\mathcal{H}_{a b}^{\dagger}$ are forbidden; in principle, one could include quartic terms in $\mathcal{T}_{a b c}^{\dagger}$, but we will not consider them here.

Thus, by analogy with WGT, the general form of $L_{\mathrm{G}}$, possessing terms no higher than quadratic order in the field strengths, is of the form

$$
L_{\mathrm{G}}=L_{\mathcal{R}^{\dagger 2}}+L_{\mathcal{H}^{\dagger 2}}
$$

where the expressions for $L_{\mathcal{R}^{\dagger 2}}$ and $L_{\mathcal{H}^{\dagger 2}}$ are given in (4), but written in terms of the eWGT field strengths, i.e., making the replacements $\mathcal{R}_{a b c d} \rightarrow \mathcal{R}_{a b c d}^{\dagger}$ and $\mathcal{H}_{a b} \rightarrow \mathcal{H}_{a b}^{\dagger}$. Performing a similar derivation to the WGT case, ${ }^{71}$ one can show that the eWGT field strength $\mathcal{R}_{a b c d}^{\dagger}$ satisfies a form of the Gauss-Bonnet identity such that the combination (59), with the replacement $\mathcal{R}_{a b c d} \rightarrow \mathcal{R}_{a b c d}^{\dagger}$, contributes a total derivative to the action (in $D \leq 4$ dimensions), and so has no effect on the resulting field equations. Hence one may set any one of $\alpha_{1}, \alpha_{3}$, or $\alpha_{6}$ in (4) to zero, without loss of generality (at least classically), but we will retain all these terms for the moment.

We note that, in the total Lagrangian (see Section III H), one may also include terms of the generic form $\phi^{2} \mathcal{R}^{\dagger}$ and $\phi^{2} L_{\mathcal{T}^{\dagger 2}}$, where $L_{\mathcal{T}^{\dagger 2}}$ is given in (3) with $\mathcal{T}_{a b c} \rightarrow \mathcal{T}_{a b c}^{\dagger}$ (but with no term proportional to $\beta_{3}$, because the eWGT torsion trace vanishes identically). Since these terms depend upon the scalar (compensator) field $\phi$, in addition to the gravitational gauge fields, we do not consider them to be part of the free gravitational action, but instead regard them as belonging to the matter Lagrangian $L_{\mathrm{M}}$.

\section{H. Field equations in eWGT}

As in WGT, the total action $S_{\mathrm{T}}$ is simply the sum of the matter and free gravitational actions. In the free gravitational sector, the form of the gravitational Lagrangian (166) induces a dependence on all three gauge fields and their first derivatives, plus a dependence on $\partial^{2} h$ (suppressing indices for brevity). The dependence of the gravitational Lagrangian on $\partial h$ makes eWGT more similar to PGT than WGT, but the dependence on $\partial^{2} h$ is unique to eWGT and arises from the term proportional to $\mathcal{H}_{a b}^{\dagger} \mathcal{H}^{\dagger a b}$ in (166). In the matter sector, covariant derivatives of the matter field $\varphi$ induce a dependence on $\varphi, \partial \varphi, h, \partial h, A$, and $V$; the dependence on $\partial h$ is now compared to WGT and arises from the term containing $\mathcal{T}_{a}$ in $\mathcal{D}_{a}^{\dagger} \varphi$. We will also consider here the inclusion of an additional "compensator" scalar field $\phi$ in the matter action and further admit the possibility that it may include a kinetic term for the scalar field that contains derivatives of $\phi$. Moreover, if the matter action includes a term proportional to $\phi^{2} \mathcal{R}^{\dagger}$, then this brings an additional dependence on $\partial A$ and $\partial V$; the latter is now compared to WGT. Finally, unlike in WGT, the inclusion of a term of the generic form $\phi^{2} L_{\mathcal{T}}$;2 produces no additional functional dependencies. Consequently, we take the 
total Lagrangian density to be

$$
\mathcal{L}_{\mathrm{T}}=\mathcal{L}_{\mathrm{G}}\left(h, \partial h, \partial^{2} h, A, \partial A, V, \partial V\right)+\mathcal{L}_{\mathrm{M}}(\varphi, \partial \varphi, \phi, \partial \phi, h, \partial h, A, \partial A, V, \partial V),
$$

where we have indicated the functional dependencies in the most general case. It is worth reiterating that these dependencies differ considerably from those in WGT, as indicated in (60).

Although $\mathcal{L}_{\mathrm{T}}$ is at most quadratic in the field strength tensors $\mathcal{R}_{a b c d}^{\dagger}(h, A, \partial A, V, \partial V), \mathcal{T}_{a b c}^{\dagger}$ $(h, \partial h, A)$, and $\mathcal{H}_{a b}^{\dagger}\left(h, \partial h, \partial^{2} h, A, \partial A, \partial V\right)$, the last of these depends (linearly) on the second derivative $\partial^{2} h$ of the translational gauge field, as indicated. Thus, unlike in WGT, if the term proportional to $\mathcal{H}^{\dagger a b} \mathcal{H}_{a b}^{\dagger}$ is included in $\mathcal{L}_{\mathrm{G}}$, then the corresponding terms it generates in the resulting field equations will typically be linear in fourth-order derivatives of $h$. We discuss this issue in detail in Section IV B.

Variation of $S_{\mathrm{T}}$ with respect to $h_{a}{ }^{\mu}, A^{a b}{ }_{\mu}$, and $V_{\mu}$ leads to three coupled gravitational field equations. As previously, it is convenient to work with quantities carrying only Latin indices and write the general field equations as

$$
\begin{aligned}
t^{a}{ }_{b}+\tau^{a}{ }_{b} & =0, \\
s_{a b}{ }^{c}+\sigma_{a b}{ }^{c} & =0, \\
j^{a}+\zeta^{a} & =0,
\end{aligned}
$$

where $t^{a}{ }_{b} \equiv t^{a}{ }_{\mu} h_{b}{ }^{\mu}, s_{a b}{ }^{c} \equiv s_{a b^{\mu}} b^{c}{ }_{\mu}$, and $j^{a} \equiv j^{\mu} b^{a}{ }_{\mu}$, and similarly for the matter sector. In the gravitational sector, $t^{a}{ }_{\mu} \equiv \delta \mathcal{L}_{\mathrm{G}} / \delta h_{a}{ }^{\mu}, s_{a b}{ }^{\mu} \equiv \delta \mathcal{L}_{\mathrm{G}} / \delta A^{a b}{ }_{\mu}$, and $j^{\mu} \equiv \delta \mathcal{L}_{\mathrm{G}} / \delta V_{\mu}$, respectively. In the matter sector, the energy-momentum $\tau^{a}{ }_{\mu} \equiv \delta \mathcal{L}_{\mathrm{M}} / \delta h_{a}{ }^{\mu}$, spin-angular-momentum $\sigma_{a b}{ }^{\mu} \equiv$ $\delta \mathcal{L}_{\mathrm{M}} / \delta A^{a b}{ }_{\mu}$, and dilation current $\zeta^{\mu} \equiv \delta \mathcal{L}_{\mathrm{M}} / \delta V_{\mu}$ of the matter fields act as sources.

All the quantities in (168a)-(168c) are clearly invariant under GCT, and it is straightforward to show that they all transform covariantly under local Lorentz rotations in accordance with their respective index structures. Under extended local dilations, the quantities in (168b) and (168c) are all covariant with weight $w=1$. Contrary to WGT, however, the individual quantities $t_{b}{ }_{b}$ and $\tau^{a}{ }_{b}$ in (168a) are not, in general, covariant under extended local dilations. This rather unusual feature is a result of the transformation law (137) for $A^{a b}{ }_{\mu}$ containing one of the other gauge fields, namely, the inverse $h$-field $b^{a}{ }_{\mu}$. Indeed, under an extended local dilation,

$$
\begin{aligned}
t_{a b}^{\prime} & =t_{a b}+2 \theta\left(s_{a c}{ }^{c} \mathcal{P}_{b}-s_{c b a} \mathcal{P}^{c}\right), \\
\tau_{a b}^{\prime} & =\tau_{a b}+2 \theta\left(\sigma_{a c}{ }^{c} \mathcal{P}_{b}-\sigma_{c b a} \mathcal{P}^{c}\right),
\end{aligned}
$$

although it is worth noting that the traces $t^{a}{ }_{a}$ and $\tau^{a}{ }_{a}$ do transform covariantly, with weight $w=0$ (i.e., invariant). Hence the non-covariant part of the transformation results only from the antisymmetric part of the energy-momentum tensor. Nonetheless, although neither $t_{a b}$ nor $\tau_{a b}$ is individually covariant, one sees that the full $h$-field equation (168a) is covariant, since the non-covariant terms in (169a) and (169b) cancel by virtue of the $A$-field equation (168b). Moreover, the transformations (169a) and (169b) suggest ${ }^{93}$ how to construct covariant forms of $t_{a b}$ and $\tau_{a b}$. From the transformation property (144) of the vector gauge field $V_{\mu}$, we see immediately that, under an extended local dilation, the quantity

$$
\tau_{a b}^{\dagger} \equiv \tau_{a b}+2 \sigma_{c b a} \mathcal{V}^{c}-2 \sigma_{a c}^{c} \mathcal{V}_{b}
$$

does transform covariantly with weight $w=0$ (i.e., it is invariant). It is also worth noting that $\tau^{\dagger a}{ }_{a}=\tau_{a}^{a}$. One may construct a covariant $t_{a b}^{\dagger}$ in a similar way. Indeed, the gravitational field equation (168a) can then be replaced by

$$
t^{\dagger a}{ }_{b}+\tau^{\dagger a}{ }_{b}=0 .
$$

Varying $S_{\mathrm{T}}$ with respect to $\varphi$ and $\phi$ leads to the matter field equations. These are easily found from those obtained for WGT in Section II H and amount to replacing $\mathcal{D}_{a}^{*}$ with $\mathcal{D}_{a}^{\dagger}$. An important difference does arise, however, since $\mathcal{T}_{a}^{\dagger} \equiv 0$. Consequently, in eWGT, one finds that the matter 
equations of motion can be written in the form

$$
\begin{aligned}
& \frac{\delta L_{\mathrm{M}}}{\delta \varphi}=\frac{\bar{\partial} L_{\mathrm{M}}}{\partial \varphi}-\mathcal{D}_{a}^{\dagger}\left(\frac{\partial L_{\mathrm{M}}}{\partial\left(\mathcal{D}_{a}^{\dagger} \varphi\right)}\right)=0, \\
& \frac{\delta L_{\mathrm{M}}}{\delta \phi}=\frac{\bar{\partial} L_{\mathrm{M}}}{\partial \phi}-\mathcal{D}_{a}^{\dagger}\left(\frac{\partial L_{\mathrm{M}}}{\partial\left(\mathcal{D}_{a}^{*} \phi\right)}\right)=0,
\end{aligned}
$$

where $\bar{\partial} L_{\mathrm{M}} / \partial \varphi \equiv\left[\partial L_{\mathrm{M}}\left(\varphi, \mathcal{D}_{a}^{\dagger} u, \phi, \mathcal{D}_{a}^{\dagger} \phi, \ldots\right) / \partial \varphi\right]_{u=\varphi}$, so that $\varphi$ and $\mathcal{D}_{a}^{\dagger} \varphi$ are treated as independent variables, and similarly for the $\phi$ field equation. This result has the important consequence that, contrary to the general case in WGT (and PGT), the matter field equations derived from the minimal-coupling procedure applied to the action $S_{\mathrm{M}}$ are equivalent to those obtained simply by applying the minimal-coupling procedure to the field equations directly, independent of the forms of $\mathcal{L}_{\mathrm{G}}$ and $\mathcal{L}_{\mathrm{M}}$. We should note, however, that the general form (172a) is only valid if the action (148) has not subsequently been modified to restore any further required invariances that were lost in the localisation of the extended Weyl symmetry.

\section{Conservation laws in eWGT}

The conservation laws for eWGT may also be straightforwardly obtained. Invariance of $S_{\mathrm{G}}$ under (infinitesimal) local Lorentz rotations, GCTs, and extended local dilations, respectively, leads (after a lengthy calculation) to the following manifestly covariant conservation laws in the free gravitational sector:

$$
\begin{array}{r}
\mathcal{D}_{c}^{\dagger}\left(h s_{a b}{ }^{c}\right)+h t_{[a b]}^{\dagger}=0, \\
\mathcal{D}_{c}^{\dagger}\left(h t^{\dagger c}{ }_{d}\right)-h\left(s_{a b}{ }^{c} \mathcal{R}^{\dagger a b}{ }_{c d}-t^{\dagger c}{ }_{b} \mathcal{T}^{\dagger b}{ }_{c d}+j^{\dagger c} \mathcal{H}_{c d}\right)=0,
\end{array}
$$

where we have defined the manifestly covariant quantity

$$
j^{\dagger a} \equiv j^{a}-2 s_{b}^{a b}
$$

Comparing these conservation laws with the equivalent results in standard WGT, we see that (68a) and (173) have analogous forms, whereas the forms (68b) and (174) differ slightly in that the latter does not contain the field strength tensor $H_{a b}^{\dagger}$ of the vector gauge field (152), but instead the simpler object $H_{a b}=h_{a}{ }^{\mu} h_{b}{ }^{\nu}\left(\partial_{\mu} V_{v}-\partial_{v} V_{\mu}\right)$, which is easily verified also to be covariant under extended local dilations; further, this object is multiplied by $j^{\dagger a}$ rather than simply by $j^{a}$. The largest difference with WGT occurs, however, when we consider invariance of $S_{\mathrm{G}}$ under (infinitesimal) local dilations, which in eWGT leads to the two further manifestly covariant identities

$$
\begin{aligned}
D_{c}^{\dagger}\left(h j^{\dagger c}\right) & =0, \\
h t^{\dagger c}{ }_{c} & =0 .
\end{aligned}
$$

Thus, whereas in WGT one obtains the single differential conservation equation (68c), in eWGT one also obtains an additional algebraic relation. The relation (177) shows that the trace of the gravitational sector's contribution to the $h$-field equation (171) vanishes. In practice, the relation (177) provides a very useful check (albeit only partial) on the derivation of the gravitational sector's contribution to the $h$-field equation.

Turning to the matter sector, the local Lorentz, GCT, and extended local dilation invariance properties of $S_{\mathrm{M}}$ lead, respectively, to the corresponding manifestly covariant identities,

$$
\begin{aligned}
\mathcal{D}_{c}^{\dagger}\left(h \sigma_{a b}{ }^{c}\right)+h \tau_{[a b]}^{\dagger}+\frac{1}{2} \frac{\delta L_{\mathrm{M}}}{\delta \varphi} \Sigma_{a b} \varphi & =0, \\
\mathcal{D}_{c}^{\dagger}\left(h \tau^{\dagger c}{ }_{d}\right)-h\left(\sigma_{a b}{ }^{c} \mathcal{R}^{\dagger a b}{ }_{c d}-\tau^{\dagger c}{ }_{b} \mathcal{T}^{\dagger b}{ }_{c d}+\zeta^{\dagger c} \mathcal{H}_{c d}\right)+\frac{\delta L_{\mathrm{M}}}{\delta \phi} \mathcal{D}_{d}^{\dagger} \phi+\frac{\delta L_{\mathrm{M}}}{\delta \varphi} \mathcal{D}_{d}^{\dagger} \varphi & =0, \\
\mathcal{D}_{c}^{\dagger}\left(h \zeta^{\dagger c}\right) & =0, \\
h \tau^{\dagger c}{ }_{c}+\frac{\delta L_{\mathrm{M}}}{\delta \phi} \phi-\frac{\delta L_{\mathrm{M}}}{\delta \varphi} w \varphi & =0,
\end{aligned}
$$


where we have defined the quantity

$$
\zeta^{\dagger a} \equiv \zeta^{a}-2 \sigma_{b}^{a b},
$$

which we will consider in more detail below. Thus, as in PGT and WGT, one is assured with the help of the matter field equations that the gravitational field equations become consistent. Moreover, the relation (178d) shows that the trace of the matter sector's contribution to the $h$-equation is simply related to the $\phi$ and $\varphi$ matter field equations; this provides a useful (partial) check on the derivation of the matter sector's contribution to the $h$-field equation. It is worth noting that the above sets of conservation laws hold for any subset of terms in $L_{\mathrm{G}}$ and $L_{\mathrm{M}}$, respectively, that is covariant with weight $w=-4$ under extended local Weyl transformations.

\section{J. Alternative variational principle in eWGT}

The identification of the covariant energy-momentum tensor (170) and its gravitational sector counterpart $t_{a b}^{\dagger}$, and the covariant currents (175) and (179), raises the question of whether these quantities may be arrived at more directly from an alternative variational principle. It transpires that this is indeed the case, and they arise naturally if one simply makes a change of field variables from the set $\varphi, \phi, h_{a}^{\mu}, A^{a b}{ }_{\mu}, V_{\mu}$, and their derivatives, with which we have worked so far, to the new set $\varphi, \phi, h_{a}^{\mu}, A^{\dagger a b}{ }_{\mu}, V_{\mu}$, and their derivatives (i.e., we replace $A^{a b}{ }_{\mu}$ by $A^{\dagger a b}{ }_{\mu}$ in the set). One should note that we are simply making a change of field variables here, rather than considering $A^{\dagger a b}{ }_{\mu}$ to be an independent field variable; in other words, we still consider $A^{\dagger a b}{ }_{\mu}$ to be given in terms of $h_{a}^{\mu}, A^{a b}{ }_{\mu}$, and $V_{\mu}$ by its defining relationship (142), rather than an independent quantity whose relationship to the other variables would be determined from the variational principle. It is worth recalling that the quantities $A^{\dagger a b}{ }_{\mu}$ (unlike $A^{a b}{ }_{\mu}$ ) transform covariantly (with weight $w=0$, i.e., invariant) under extended local dilations, and so we might expect a more straightforward expression of the covariance of $\mathcal{L}_{\mathrm{M}}$ and $\mathcal{L}_{\mathrm{G}}$ when they are written in terms of $A^{\dagger a b}{ }_{\mu}$ rather than $A^{a b}{ }_{\mu}$.

On making this change of field variables, a significant simplification does indeed occur. This is most easily demonstrated by introducing the derivative operators (see Appendix E)

$$
\mathcal{D}_{a}^{\natural} \varphi \equiv h_{a}{ }^{\mu} D_{\mu}^{\natural} \varphi \equiv h_{a}{ }^{\mu}\left(\partial_{\mu}+\frac{1}{2} A^{\dagger b c}{ }_{\mu} \Sigma_{b c}\right) \varphi .
$$

One should note that these derivatives do not transform covariantly under extended local dilations. Nonetheless, by analogy with (154), one can still define the quantities

$$
\mathcal{T}^{\natural a}{ }_{b c} \equiv h_{b}{ }^{\mu} h_{c}{ }^{v}\left(D_{\mu}^{\natural} b^{a}{ }_{\nu}-D_{\nu}^{\natural} b^{a}{ }_{\mu}\right)
$$

and the corresponding trace $\mathcal{T}_{b}^{\natural} \equiv \mathcal{T}^{\natural a}{ }_{b a}$. It is straightforward to show that $\mathcal{T}_{b}^{\natural}=\mathcal{T}_{b}+3 \mathcal{V}_{b}$, where $\mathcal{T}_{b}$ is the trace of the PGT torsion. Hence the full eWGT covariant derivative can be written as

$$
\mathcal{D}_{a}^{\dagger} \varphi=\left(\mathcal{D}_{a}^{\natural}-\frac{1}{3} w \mathcal{T}_{a}^{\natural}\right) \varphi,
$$

which is expressible wholly in terms of the fields $h, \partial h$, and $A^{\dagger}$ (suppressing indices). Since (182) does not depend explicitly on the dilation gauge field $V_{\mu}$ or its derivatives, then neither will any Lagrangian constructed from eWGT covariant derivatives or field strengths, when working in terms of the new set of field variables. Thus, the functional dependencies in (167) become

$$
\mathcal{L}_{\mathrm{T}}=\mathcal{L}_{\mathrm{G}}\left(h, \partial h, \partial^{2} h, A^{\dagger}, \partial A^{\dagger}\right)+\mathcal{L}_{\mathrm{M}}\left(\varphi, \partial \varphi, \phi, \partial \phi, h, \partial h, A^{\dagger}, \partial A^{\dagger}\right),
$$

in which there is no explicit dependence on the dilation gauge field $V_{\mu}$ or its derivatives. Thus, eWGT can be expressed in terms of just the two combinations $h$ and $A^{\dagger}$ of the gauge fields. In this respect, eWGT is structurally more similar to PGT, whilst possessing (a more general version of) the local scale invariance of WGT.

Working in terms of the new field variables, $\mathcal{L}_{\mathrm{T}}$ is at most quadratic in the field strength tensors $\mathcal{R}_{a b c d}^{\dagger}\left(h, A^{\dagger}, \partial A^{\dagger}\right), \mathcal{T}_{a b c}^{\dagger}\left(h, \partial h, A^{\dagger}\right)$, and $\mathcal{H}_{a b}^{\dagger}\left(h, \partial h, \partial^{2} h, A^{\dagger}, \partial A^{\dagger}\right)$, where once again the last of these depends (linearly) on the second derivative $\partial^{2} h$ of the translational gauge field, as indicated. Hence, the term proportional to $\mathcal{H}_{a b}^{\dagger} \mathcal{H}^{\dagger a b}$ in $\mathcal{L}_{\mathrm{G}}$ (if included) will again generate terms in the equations 
of motion that are typically linear in fourth-order derivatives of $h$. As mentioned previously, we discuss this issue in detail in Section IV B.

As a notational convenience, when working with the new set of field variables, we denote the variational derivative of a Lagrangian $\mathcal{L}$ with respect to any one of the fields $\chi$ by $(\delta \mathcal{L} / \delta \chi)_{\dagger}$ to distinguish it from the variational derivative $\delta \mathcal{L} / \delta \chi$ obtained previously using the original set of field variables. Straightforward (but lengthy) application of the chain rule for partial derivatives shows that for (say) the matter Lagrangian one obtains

$$
\begin{aligned}
\left(\frac{\delta \mathcal{L}_{\mathrm{M}}}{\delta h_{c}{ }^{\mu}}\right)_{\dagger} & =\tau^{c}{ }_{\mu}+2 \sigma_{a b}{ }^{c} \mathcal{V}^{a} b_{\mu}^{b}-2 \sigma^{c b}{ }_{b} V_{\mu}=\tau^{\dagger c}{ }_{\mu}, \\
\left(\frac{\delta \mathcal{L}_{\mathrm{M}}}{\delta A^{\dagger a b}{ }_{\mu}}\right)_{\dagger} & =\sigma_{a b}{ }^{\mu}, \\
\left(\frac{\delta \mathcal{L}_{\mathrm{M}}}{\delta V_{\mu}}\right)_{\dagger} & =\zeta^{\mu}-2 h_{a}{ }^{\mu} \sigma^{a b}{ }_{b}=\zeta^{\dagger \mu}=0, \\
\left(\frac{\delta \mathcal{L}_{\mathrm{M}}}{\delta \varphi}\right)_{\dagger} & =\frac{\delta \mathcal{L}_{\mathrm{M}}}{\delta \varphi}, \\
\left(\frac{\delta \mathcal{L}_{\mathrm{M}}}{\delta \phi}\right)_{\dagger} & =\frac{\delta \mathcal{L}_{\mathrm{M}}}{\delta \phi},
\end{aligned}
$$

and directly analogous results hold for the free gravitational Lagrangian. Thus we see that straightforward variation with respect to the new set of field variables leads directly to the covariant energy-momentum tensor (170) and the covariant current (175) (and their counterparts in the free gravitational sector) that we identified earlier; the latter of which always vanishes. We note further that the spin-angular momentum tensor $\sigma_{a b}{ }^{\mu}$ and the variational derivatives with respect to $\varphi$ and $\phi$ (and their counterparts from the gravitational sector) are not altered by working in terms of the new set of field variables. Indeed, the results (184a)-(184e) (and their counterparts for the free gravitational sector) lead to valuable short-cuts in calculating the equations of motion in terms of either the old or new set of variables, since holding $A^{\dagger a b}{ }_{\mu}$ constant in the derivatives (rather than just $A^{a b}{ }_{\mu}$, as done previously) vastly reduces the number of terms to be calculated.

The relation $(184 \mathrm{c})$ that defines the covariant dilation current is worthy of further comment. Since the matter Lagrangian does not depend explicitly on the dilation gauge field $V_{\mu}$ or its derivative, then $\zeta^{\dagger \mu}=0$ and so

$$
\zeta^{a}=2 \sigma_{b}^{a b}
$$

and similarly for the free gravitational Lagrangian, so

$$
j^{a}=2 s^{a b}{ }_{b}
$$

Thus, when working in terms of the original set of fields, the contribution of the matter sector to the $V$-field equation (168c) is merely twice the relevant contraction of its contribution to the $A$-field equation (168b). The result (186) shows that the same is true for the gravitational sector. Finally, we note that, combining the relations (177) and (178d) and the relations (185) and (186) shows that the resulting theory has only three independent field equations, namely, the $h$-equation (171), the $A$-equation (or, equivalently, the $A^{\dagger}$-equation) (168b), and the $\varphi$-equation (172a). In this respect, eWGT is again more similar to PGT than standard WGT.

For the remainder of this paper, unless otherwise stated, we will work in terms of the original set of fields $\varphi, \phi, h_{a}{ }^{\mu}, A^{a b}{ }_{\mu}, V_{\mu}$, and their derivatives, but we will use the relations (184a) and (184c) to simplify our calculations. Moreover, we will use the relations (177), (178d), (185), and (186) as a check on our derivations of the two independent gravitational field equations (171) and (168b). 


\section{K. Dirac matter field in eWGT}

To illustrate the coupling of matter to the gravitational gauge fields in eWGT, we again consider a Dirac field and begin with the special-relativistic Lagrangian (70), which was the starting point for our discussion in Section II J. Following our general procedure for constructing a matter action (148) that is invariant under extended Weyl transformations and applying it to (70), the appropriate form for the corresponding Lagrangian density is

$$
\mathcal{L}_{\mathrm{D}}=h^{-1} L_{\mathrm{D}}=h^{-1}\left(\frac{1}{2} i \bar{\psi} \gamma \gamma^{a} \stackrel{\leftrightarrow}{\mathcal{D}_{a}^{\dagger}} \psi-\mu \phi \bar{\psi} \psi\right),
$$

which is simply the WGT Lagrangian density (71), but with $\mathcal{D}_{a}^{*} \rightarrow \mathcal{D}_{a}^{\dagger}$. Similar to WGT, since both $\psi$ and $\bar{\psi}$ have Weyl weight $w=-\frac{3}{2}$, the dilation gauge field $V_{\mu}$ interacts in the same manner with each of them, thereby ruling out the interpretation of $V_{\mu}$ as the electromagnetic potential. Moreover, $V_{\mu}$ similarly vanishes completely from the action (187). Thus, as occurred in WGT, (187) is identical to the covariant Dirac Lagrangian in PGT, given in (72). In other words, the kinetic term in the Dirac action in PGT is already also invariant to extended local dilations. Indeed, this was one of our original motivations for exploring eWGT.

Varying the action corresponding to (187) with respect to $\bar{\psi}$, one obtains a field equation of the form (172a) (with $\varphi$ replaced by $\bar{\psi}$ ), which is immediately found to read

$$
i \gamma^{a} \mathcal{D}_{a}^{\dagger} \psi-\mu \phi \psi=0
$$

An equivalent adjoint field equation in $\bar{\psi}$ is obtained by varying the action with respect to $\psi$. In particular, we verify that the field equation (188) derived from minimal coupling at the level of the action is equivalent to that which would be obtained simply by applying the minimal-coupling procedure to the field equation directly, independent of the form of $\mathcal{L}_{\mathrm{G}}$. Since the Dirac Lagrangian density (187) may also be expressed as (72), however, one can rewrite (188) more simply in terms of PGT quantities as in (74). The covariance of (74) under extended local dilations is easily checked directly by noting that $\gamma^{a}\left(\mathcal{D}_{a}+\frac{1}{2} \mathcal{T}_{a}\right) \psi=\gamma^{a} \mathcal{D}_{a}^{\dagger} \psi$, where we have used the fact that for a Dirac spinor $w=-\frac{3}{2}$ and $\gamma^{a} \Sigma_{b a}=\frac{1}{4} \gamma^{a}\left[\gamma_{b}, \gamma_{a}\right]=-\frac{3}{2} \gamma_{b}$. We also note that (188) and (74) are valid for any choice of the gravitational Lagrangian density $\mathcal{L}_{\mathrm{G}}$, but the expression for $\mathcal{T}_{a}$ in (74) will depend on the form of $\mathcal{L}_{\mathrm{G}}$.

Following the discussion in Section II J, one may rewrite (74), and hence (188), in terms of the reduced PGT covariant derivative as (76), which again reveals that only the total antisymmetric part of the PGT "torsion" explicitly affects the dynamics of the Dirac field $\psi$. Nonetheless, unlike in WGT, one must note that the presence of torsion (even in the case $\mathcal{T}_{[a b c]}=0$ ) will still induce physical effects on $\psi$, since the PGT torsion enters into the definition of $\mathcal{R}_{a b c d}^{\dagger}$ which depend on the gravitational field equations that determine the $h$ and $B$ fields. Alternatively, one can rewrite (188) in manifestly eWGT-covariant form in terms of the reduced eWGT covariant derivative, and one quickly finds that (76) is again satisfied, but with the replacements ${ }^{0} \mathcal{D}_{a} \rightarrow{ }^{0} \mathcal{D}_{a}^{\dagger}$ and $\mathcal{T}_{[a b c]} \rightarrow \mathcal{T}_{[a b c]}^{\dagger}$. Thus, only the total antisymmetric part of the eWGT "torsion" explicitly affects the dynamics of the $\psi$ field.

From (76), the energy-momentum and spin-angular-momentum tensors of the massive Dirac field $\psi$ are again given by the expressions (77) and (78), respectively. The expression (78) for the spin-angular-momentum tensor $\sigma_{a b}{ }^{c}$ is already manifestly eWGT covariant. For the energymomentum tensor $\tau_{b}^{a}$, one may show that (77) can be rewritten as

$$
\tau_{b}^{a}=\frac{1}{2} i h^{-1} \bar{\psi} \gamma^{a} \stackrel{\leftrightarrow}{\mathcal{D}_{b}^{\dagger}} \psi-\delta_{b}^{a} \mathcal{L}_{\mathrm{D}}-2 \sigma_{c b}{ }^{a} \mathcal{V}^{c},
$$

but, as anticipated from our discussion in Section III $\mathrm{H}$, this quantity is not covariant with respect to extended local dilations. Indeed, one may show that $\tau^{a}{ }_{b}$ transforms as

$$
\tau^{\prime a}{ }_{b}=\tau_{b}^{a}-2 \theta \sigma_{c d}{ }^{a} \mathcal{P}^{c} \delta_{b}^{d} .
$$

Recalling that $\sigma_{a b}{ }^{b}=0$ for Dirac matter, the transformation (190) agrees with the general result (169b). Following the discussion in Section III H, however, we can construct the eWGT-covariant 
energy-momentum tensor

$$
\tau_{b}^{\dagger a}=\tau_{b}^{a}+2 \sigma_{c b}{ }^{a} \mathcal{V}^{c} .
$$

Finally, we note that one might also consider adding further terms to the matter Lagrangian density (72) in an analogous manner to that discussed in Section II J for WGT. Hence, we base our massive Dirac field matter Lagrangian on (79), but appropriately generalised to be eWGT covariant. Thus, in the most general case, one might consider

$$
\mathcal{L}_{\mathrm{M}}=h^{-1}\left[\frac{1}{2} i \bar{\psi} \gamma \gamma^{a} \stackrel{\leftrightarrow}{\mathcal{D}}_{a} \psi-\mu \phi \bar{\psi} \psi+\frac{1}{2} \nu\left(\mathcal{D}_{a}^{\dagger} \phi\right)\left(\mathcal{D}^{\dagger a} \phi\right)-\lambda \phi^{4}-a \phi^{2} \mathcal{R}^{\dagger}+\phi^{2} L_{\mathcal{T}^{\dagger 2}}\right],
$$

where $\mu, v, \lambda$, and $a$ are dimensionless constants (usually positive), and there are two further dimensionless constants $\beta_{1}$ and $\beta_{2}$ in $L_{\mathcal{T}}{ }^{\dagger 2}$. It is worth noting that $\mu \phi$ has the dimensions of mass in natural units.

\section{Electromagnetic field in eWGT}

The dynamics of the electromagnetic field in eWGT follow by direct analogy from our discussion in Section II K in the context of WGT, with the replacements $\widehat{\mathcal{F}}_{a b}^{*} \rightarrow \widehat{\mathcal{F}}_{a b}^{\dagger}, \mathcal{T}^{* c}{ }_{a b} \rightarrow \mathcal{T}^{\dagger c}{ }_{a b}$, and $\mathcal{D}_{a}^{*} \rightarrow \mathcal{D}_{a}^{\dagger}$, and recalling that $\mathcal{T}_{a}^{\dagger} \equiv 0$. Thus, as in WGT (and PGT), one arrives at the conclusion that the appropriate Lagrangian density for the EM field is given by (83), namely,

$$
\mathcal{L}_{\mathrm{M}}=h^{-1} L_{\mathrm{M}}=-h^{-1}\left(\frac{1}{4} \mathcal{F}_{a b} \mathcal{F}^{a b}+\mathcal{J}^{a} \mathcal{A}_{a}\right),
$$

which has an overall Weyl weight of zero, as required, provided $w\left(\mathcal{J}_{a}\right)=-3$. Hence, the electromagnetic Lagrangian in PGT is already covariant under extended local dilations, which again was one of our original motivations for exploring eWGT.

Since the Lagrangian (193) is identical to that in PGT, then the equation of motion for the electromagnetic field is again given by (86), which may be straightforwardly recast as

$$
\mathcal{D}_{a}^{\dagger} \mathcal{F}^{a c}-\frac{1}{2} \mathcal{T}^{\dagger c}{ }_{a b} \mathcal{F}^{a b}=\mathcal{J}^{c},
$$

which is manifestly eWGT covariant. One should note that this differs slightly from the corresponding form (84) in WGT, since $\mathcal{T}_{a}^{\dagger} \equiv 0$. Also, the differential identity (87) satisfied by the electromagnetic field strength tensor may also directly be shown to be covariant under local dilations, since it can be easily rewritten as

$$
\mathcal{D}_{[a}^{\dagger} \mathcal{F}_{b c]}-\mathcal{T}_{[a b}^{\dagger d} \mathcal{F}_{c] d}=0
$$

Since (86) and (87) also hold in eWGT, then so too do the alternative forms given in (89) in terms of the reduced covariant derivative in PGT, which show that the PGT torsion plays no explicit role in the dynamics of the electromagnetic field. One may also rewrite (89) in terms of the reduced eWGT covariant derivative, and one finds that (89) is again satisfied, but with the replacement ${ }^{0} \mathcal{D}_{a} \rightarrow{ }^{0} \mathcal{D}_{a}^{\dagger}$. Thus the eWGT torsion similarly has no direct effect of the EM field dynamics. As mentioned above for the Dirac field, however, the presence of torsion will still induce physical effects (unlike in WGT), since the reduced covariant derivative ${ }^{0} \mathcal{D}_{a}^{\dagger}$ depends on the gauge fields, which are determined by gravitational field equations; these, in turn, depend on the "curvative" tensor $\mathcal{R}_{a b c d}^{\dagger}$ or its contractions, which contain the torsion.

The energy-momentum tensor $\tau^{a}{ }_{b}$ of the EM field has the same form as in PGT and WGT, namely, that given in (90), which is already manifestly eWGT covariant. Indeed, from (170), one sees immediately that $\tau_{b}^{\dagger a}=\tau_{b}^{a}$, since the EM field spin-angular-momentum tensor $\sigma_{a b}{ }^{c}$ vanishes.

Finally, one may straightforwardly include the interaction of the electromagnetic and Dirac fields, both of which are coupled to gravity, in the same way as in WGT, as discussed in Section II K. 


\section{Einstein gauge and scale-invariant variables}

As was the case for WGT, the forms of the field equations in eWGT can be simplified considerably by using the scale gauge freedom to impose the Einstein gauge, in which the scalar field is set to a constant everywhere, $\phi=\phi_{0}$ (provided $\phi$ does not vanish). Moreover, as occurred for WGT (see Section II L), we show here that the resulting field equations are identical in form to those obtained when working in terms of a new set of scale-invariant field variables. Thus, this again provides an alternative interpretation of the Einstein gauge, since the approach using scale-invariant variables involves no breaking of the scale symmetry.

It is convenient to work in terms of the alternative set of field variables $\varphi, \phi, h_{a}^{\mu}$, and $A^{\dagger a b}{ }_{\mu}$ discussed in Section III J, in which the total Lagrangian density $\mathcal{L}_{\mathrm{T}}$ does not depend explicitly on the dilational gauge field $V_{\mu}$ or its derivatives. We begin by introducing the new scale-invariant matter field and gravitational gauge fields

$$
\widehat{\varphi} \equiv\left(\frac{\phi}{\phi_{0}}\right)^{-w} \varphi, \quad \widehat{h}_{a}^{\mu} \equiv\left(\frac{\phi}{\phi_{0}}\right)^{-1} h_{a}{ }^{\mu}, \quad \widehat{A}_{\mu}^{\dagger a b} \equiv A_{\mu}^{\dagger a b},
$$

where $w$ is the Weyl weight of the original matter field $\varphi$. It is also convenient to define the further scale-invariant variables $\widehat{\mathcal{A}}^{\dagger a b} \equiv \widehat{h}_{a}^{\mu} \widehat{A}_{\mu}^{\dagger a b}$.

As shown in Section III J, the eWGT covariant derivative of some general field $\chi$ with Weyl weight $w$ can be written in the form $\mathcal{D}_{a}^{\dagger} \chi=\left(\mathcal{D}_{a}^{\natural}-\frac{1}{3} w \mathcal{T}_{a}^{\natural}\right) \chi$, where $\mathcal{D}_{a}^{\natural}$ and $\mathcal{T}_{a}^{\natural}$ are defined in (180) and (181), respectively. In an analogous manner, we may define the quantities $\widehat{\mathcal{D}}_{a}^{\natural}$ and $\widehat{\mathcal{T}}_{a}^{\natural}$, for which each occurrence of $h_{a}{ }^{\mu}$ and $A^{\dagger a b}{ }_{\mu}$ in (E2) and (181) is replaced by its scale-invariant counterpart $\widehat{h}_{a}^{\mu}$ and $\widehat{A}_{\mu}^{\dagger a b}$, respectively. Thus, $\widehat{\mathcal{D}}_{a}^{\natural}=\widehat{h}_{a}^{\mu} D_{\mu}^{\natural}$ and a short calculation shows that $\widehat{\mathcal{T}}_{a}^{\natural}$ transforms covariantly with Weyl weight $w=0$ under local dilations, i.e., it is scale-invariant. One then immediately finds that the eWGT covariant derivative of the scalar field $\phi$ may be written as $\mathcal{D}_{a}^{\dagger} \phi=\frac{1}{3}\left(\phi^{2} / \phi_{0}\right) \widehat{\mathcal{T}}_{a}^{\natural}$, whereas the covariant derivative of some general field $\chi$ with Weyl weight $w$ may be written as

$$
\mathcal{D}_{a}^{\dagger} \chi=\left(\frac{\phi}{\phi_{0}}\right)^{1-w} \widehat{\mathcal{D}}_{a}^{\dagger} \widehat{\chi},
$$

where $\hat{\chi}=\left(\phi / \phi_{0}\right)^{-w} \chi$ and we have defined the derivative operator $\widehat{\mathcal{D}}_{a}^{\dagger}=\widehat{\mathcal{D}}_{a}^{\natural}-\frac{1}{3} w \widehat{\mathcal{T}}_{a}^{\natural}$, which preserves the Weyl weight of the quantity on which it acts. From (197), one sees that, aside from the overall multiplicative factor $\left(\phi / \phi_{0}\right)^{1-w}$, the scale-invariant quantity $\widehat{\mathcal{D}}_{a}^{\dagger} \widehat{\chi}$ has the same functional dependency on $\widehat{\chi}, \widehat{h}_{a}^{\mu}$, and $\widehat{A}_{\mu}^{\dagger a b}$, respectively, as $\mathcal{D}_{a}^{\dagger} \chi$ does on $\chi, h_{a}{ }^{\mu}$, and $A^{\dagger a b}{ }_{\mu}$. Similarly, one also quickly finds that the eWGT gauge field strengths defined in (41) may be written as $\mathcal{R}^{\dagger a b}{ }_{c d}=\left(\phi / \phi_{0}\right)^{2} \widehat{\mathcal{R}}_{c d}^{\dagger a b}, \mathcal{T}^{\dagger a}{ }_{b c}=\left(\phi / \phi_{0}\right) \widehat{\mathcal{T}}^{\dagger a}{ }_{b c}$, and $\mathcal{H}_{a b}^{\dagger}=\left(\phi / \phi_{0}\right)^{2} \widehat{\mathcal{H}}_{a b}^{\dagger}$, where each quantity with a caret is scale-invariant and has the same functional dependence on $\widehat{h}_{a}^{\mu}$ and $\widehat{A}_{\mu}^{\dagger a b}$ as the corresponding original quantity does on $h_{a}{ }^{\mu}$ and $A^{\dagger a b}{ }_{\mu}$, respectively.

Thus, noting that $h^{-1}=\left(\phi / \phi_{0}\right)^{-4} \widehat{h}^{-1}$, one is led to the important conclusion, analogous to that discussed in Section II L for WGT, that the Lagrangian density $\mathcal{L}_{\mathrm{T}}$ in (183) may be written as (suppressing indices for brevity)

$$
\mathcal{L}_{\mathrm{T}}=\mathcal{L}_{\mathrm{G}}\left(\widehat{h}, \partial \widehat{h}, \partial^{2} \widehat{h}, \widehat{A}^{\dagger}, \partial \widehat{A}^{\dagger}\right)+\mathcal{L}_{\mathrm{M}}\left(\widehat{\varphi}, \partial \widehat{\varphi}, \phi_{0}, 0, \widehat{h}, \partial \widehat{h}, \widehat{A}^{\dagger}, \partial \widehat{A}^{\dagger}\right) .
$$

Specifically, when written in terms of the scale-invariant field variables, the Lagrangian density $\mathcal{L}_{\mathrm{T}}$ (indeed each term separately) has the same functional form as it does in terms of the original variables with $\phi=\phi_{0}$. Thus, if $\chi$ represents $\varphi, h_{a}{ }^{\mu}$, or $A^{\dagger a b}{ }_{\mu}$, one may immediately conclude that (each term in) the equation of motion $\delta \mathcal{L}_{\mathrm{T}} / \delta \widehat{\chi}=0$ has the same functional form as (the corresponding term in) $\delta \mathcal{L}_{\mathrm{T}} /\left.\delta \chi\right|_{\phi=\phi_{0}}=0$, but with $\varphi, h_{a}{ }^{\mu}$, and $A^{\dagger a b}{ }_{\mu}$ replaced by their scale-invariant counterparts (196).

As mentioned in Section III J, the equations of motion for the $\varphi, h$, and $A$ (or $A^{\dagger}$ ) fields are the only three independent field equations of eWGT. One can also obtain equation of motion for the $V$ and $\phi$ fields, but the relations (177), (178d), (185), and (186) show that these correspond merely 
to contractions of the $A$-equation and $h$-equation, respectively. Thus, somewhat more straightforwardly than in WGT, one may immediately conclude that the equivalence discussed above also holds for the $V$ and $\phi$ equations of motion.

It is worth mentioning that one may arrive at similar conclusions to those reached above, if one instead begins by working in terms of the "standard" set of field variables $\varphi, \phi, h_{a}^{\mu}, A^{a b}{ }_{\mu}$, and $V_{\mu}$, rather than the alternative set introduced in Section III J. In this case, in place of (196), one introduces the scale-invariant matter field and gravitational gauge fields

$$
\begin{aligned}
\widehat{\varphi} & \equiv\left(\frac{\phi}{\phi_{0}}\right)^{-w} \varphi, \\
\widehat{h}_{a}^{\mu} & \equiv\left(\frac{\phi}{\phi_{0}}\right)^{-1} h_{a}{ }^{\mu}, \\
\widehat{A}_{\mu}^{\dagger a b} & \equiv A^{a b}{ }_{\mu}+\left(\mathcal{V}^{a} b^{b}{ }_{\mu}-\mathcal{V}^{b} b^{a}{ }_{\mu}\right), \\
\widehat{V}_{\mu} & \equiv V_{\mu}+\frac{1}{3} T_{\mu}+\partial_{\mu} \ln \left(\frac{\phi}{\phi_{0}}\right),
\end{aligned}
$$

where it is clear from the last definition that $\widehat{V}_{\mu}=\frac{1}{3} \widehat{T}_{\mu}^{\natural}$. It is also convenient to define the further scale-invariant variables $\widehat{\mathcal{A}}^{\dagger a b}{ }_{c} \equiv \widehat{h}_{a}{ }^{\mu} \widehat{A}_{\mu}^{\dagger a b}$ and $\widehat{V}_{a} \equiv \widehat{h}_{a}{ }^{\mu} \widehat{V}_{\mu}$. The situation is, however, slightly different to that outlined above. Specifically, the equations of motion in terms of $\varphi, h_{a}^{\mu}, A^{a b}{ }_{\mu}$, and $V_{\mu}$ arrived at by adopting the Einstein gauge must first be rewritten in terms of $\varphi, h_{a}^{\mu}$, and the combinations $A_{\mu}^{\dagger a b}$ and $V_{\mu}+\frac{1}{3} T_{\mu}$ (which will always be possible). Only then they will be identical in form to the full field equations written in terms of the scale-invariant variables $\widehat{\varphi}, \widehat{h}_{a}^{\mu}, \widehat{A}_{\mu}^{\dagger a b}$, and $\widehat{V}_{\mu}$, respectively.

\section{N. Motion of test particles in eWGT}

To determine the equation of motion of a massive test particle in eWGT, we follow the procedure outlined in Section II M for WGT, but modify it to accommodate extended local scale-invariance. Thus, we again adopt the fully classical point particle action (96), in which the Weyl weights of the dynamical variables, namely, the particle 4-momentum $p^{a}(\lambda)$, 4-velocity $v^{a}(\lambda)$, and the einbein $e(\lambda)$ along the worldline parameterised by $\lambda$, are the same as in WGT, so that the action is scale-invariant.

Varying the action (96) with respect to the three dynamical variables, respectively, one again obtains the equations of motion (97)-(99). In this case, however, it is straightforward to show that (98) can be written in a manifestly eWGT-covariant manner as $v^{c}\left(\mathcal{D}_{c}^{\dagger} p_{a}-\mathcal{T}_{c a b}^{\dagger} p^{b}\right)=e \mu^{2} \phi \mathcal{D}_{a}^{\dagger} \phi$. By analogy with our treatment in WGT, one may rewrite this equation in terms of the reduced eWGT covariant derivative, defined in (159), as $v^{c 0} \mathcal{D}_{c}^{\dagger} p_{a}=e \mu^{2} \phi^{0} \mathcal{D}_{a}^{\dagger} \phi$, which is again manifestly eWGT-covariant. As in WGT, this takes its simplest form when one chooses $e=1 /(\mu \phi)$, in which case $v^{2}=1$ and $\lambda$ corresponds to the proper time $\tau$ along the worldline; in this case the equation of motion becomes

$$
\phi v^{b 0} \mathcal{D}_{b}^{\dagger} v_{a}=\left(\delta_{a}^{b}-v_{a} v^{b}\right)^{0} \mathcal{D}_{b}^{\dagger} \phi .
$$

Once again, for ease of calculation, this may be rewritten in terms of the reduced PGT-covariant derivative to yield (103), which is also eWGT covariant, but not manifestly so. Precisely as in WGT, if one uses local scale invariance to impose the Einstein gauge $\phi=\phi_{0}$ (a constant), then (103) reduces to same result as that given in (104) for WGT, which again describes the gauge theory equivalent of geodesic motion. Unlike in WGT, however, the presence of PGT torsion may still induce physical effects, since the $h$ and $B$ fields are determined by gravitational field equations that depend on the curvature tensor $\mathcal{R}_{a b c d}^{\dagger}$, into which the PGT torsion enters.

As in WGT, by setting $\mu=0$ in the action (96) and choosing the einbein $e=1$, such that $v^{2}=0$, one finds that the equation of motion in the massless case (for example, a massless neutrino) is also given by (104), even without imposing the Einstein gauge. One may also arrive at a similar conclusion for the motion of photons by directly considering the dynamics of the electromagnetic field. 
As discussed in Section III L, the EM field tensor in eWGT again satisfies the field equation and Bianchi identity given in (89), which have precisely the same form as those obtained in the absence of torsion. Consequently, one may immediately infer that the equation of motion for photons is also given by (104), so that they too follow the gauge theory equivalent of geodesic motion.

Finally, as in WGT, the imposition of the Einstein gauge is not necessary to arrive at the geodesic equation of motion (104). One may again introduce the set of scale-invariant variables (105) and rewrite the action (96) in the form (106). Thus, one arrives once more at the geodesic equation of motion (107), which is written entirely in terms of scale-invariant variables.

\section{O. Reduced eWGT}

In Section III E, we introduced the "reduced" eWGT covariant derivative operator ${ }^{0} D_{\mu}^{\dagger}$ in (159), to which the "full" eWGT covariant derivative (143) reduces in the case that the eWGT torsion $\mathcal{T}_{a b c}^{\dagger}$ vanishes (which is a properly eWGT-covariant condition). In the context of eWGT, the term "reduced" refers to versions of quantities in which, by construction, the expression (158) holds with $\mathcal{T}_{a b c}^{\dagger} \equiv 0$ (and hence $\mathcal{K}_{a b c}^{\dagger} \equiv 0$ ). In contrast to WGT, however, such quantities still depend on the rotational gauge field $A$ and hence cannot be written entirely in terms of the other gauge fields. Such quantities are again denoted by a zero superscript preceding the kernel letter, but are perhaps less deserving of the description "reduced" than their counterparts in WGT (or PGT; see Appendix B).

Nonetheless, as was the case in WGT, one can use the covariant derivative ${ }^{0} D_{\mu}^{\dagger}$ to build an alternative class of scale-invariant gravitational gauge theories, which we term "reduced eWGT." They correspond mathematically to imposing the condition of vanishing eWGT torsion directly at the level of the action, but differ from reduced WGT in that they still depend on all the gravitational gauge fields, in particular the $A$ gauge field.

In the usual manner, one begins by defining the "reduced" gauge field strengths by considering their commutator, which gives

$$
\left[{ }^{0} D_{\mu}^{\dagger},{ }^{0} D_{\nu}^{\dagger}\right] \varphi=\frac{1}{2}^{0} R^{\dagger a b}{ }_{\mu \nu} \Sigma_{a b} \varphi+w H_{\mu \nu}^{\dagger} \varphi,
$$

where we have defined the "reduced" field strength tensor ${ }^{0} R^{\dagger a b}{ }_{\mu \nu}\left(h, \partial h, \partial^{2} h, A, \partial A, V, \partial V\right)$, which is again given by the formula (39), but with $A^{a b}{ }_{\mu}$ replaced by ${ }^{0} A^{\dagger a b}{ }_{\mu}$. Unlike in WGT, however, ${ }^{0} R^{\dagger a b}{ }_{\mu \nu}$ depends on all the gauge fields and their derivatives, most particularly the $A$ gauge field; indeed its functional dependencies are more complicated than the "full" field strength tensor $R^{\dagger a b}{ }_{\mu \nu}(h, A, \partial A, V, \partial V)$. Considering instead the commutator of "reduced" generalised covariant derivatives, one obtains

$$
\left[{ }^{0} \mathcal{D}_{c}^{\dagger},{ }^{0} \mathcal{D}_{d}^{\dagger}\right] \varphi=\frac{1}{2}^{0} \mathcal{R}^{\dagger a b}{ }_{c d} \Sigma_{a b} \varphi+w \mathcal{H}_{c d}^{\dagger} \varphi,
$$

where ${ }^{0} \mathcal{R}^{\dagger a b}{ }_{c d}=h_{c}{ }^{\mu} h_{d}{ }^{\mu 0} R^{\dagger a b}{ }_{\mu \nu}$, and the commutator (202) has no term containing a "translational" field strength of the $h$ gauge field (or "torsion"), since ${ }^{0} \mathcal{T}^{\dagger a}{ }_{b c} \equiv h_{b}{ }^{\mu} h_{c}{ }^{\nu}\left({ }^{0} D_{\mu}^{\dagger} b^{a}{ }_{\nu}-\right.$ $\left.{ }^{0} D_{\nu}^{\dagger} b^{a}{ }_{\mu}\right)=0$. As one might expect, the Bianchi identities satisfied by the reduced field strength tensors are identical to those given in Section III F, but with the replacements $\mathcal{D}_{a}^{\dagger} \rightarrow{ }^{0} \mathcal{D}_{a}^{\dagger}, \mathcal{R}_{a b c d} \rightarrow$ ${ }^{0} \mathcal{R}_{a b c d}^{\dagger}$, and $\mathcal{T}_{a b c}^{\dagger} \rightarrow{ }^{0} \mathcal{T}_{a b c}^{\dagger}=0$.

Since the "full" generalised covariant derivative is given in terms of the "reduced" one by (160), it follows that the field strength tensor $\mathcal{R}^{\dagger a b}{ }_{c d}$ appearing in (153), and its contractions, may be written in the forms (110a)-(110c), but with the replacements on the RHS ${ }^{0} \mathcal{R}^{a b}{ }_{c d} \rightarrow{ }^{0} \mathcal{R}^{\dagger a b}{ }_{c d}$, ${ }^{0} \mathcal{D}_{a} \rightarrow{ }^{0} \mathcal{D}_{a}^{\dagger}, \mathcal{K}_{a b c} \rightarrow \mathcal{K}_{a b c}^{\dagger}$, and $\mathcal{T}_{a b c} \rightarrow \mathcal{T}_{a b c}^{\dagger}$ and recalling that $\mathcal{T}_{a}^{\dagger} \equiv 0$.

For reduced eWGT, the free gravitational Lagrangian density $\mathcal{L}_{\mathrm{G}}=h^{-1} L_{\mathrm{G}}$, where by analogy with (166),

$$
L_{\mathrm{G}}=L_{\mathcal{R}^{\dagger 2}}+L_{\mathcal{H}^{\dagger 2}},
$$

which is based on (4) with $\mathcal{R}^{a b}{ }_{c d} \rightarrow{ }^{0} \mathcal{R}^{\dagger a b}{ }_{c d}$ and $\mathcal{H}_{a b} \rightarrow{ }^{0} \mathcal{R}^{\dagger} a b$. As was the case for WGT, one can simplify (203) still further (in $D \leq 4$ dimensions), since ${ }^{0} \mathcal{R}_{a b c d}^{\dagger}$ and its contractions also satisfy a Gauss-Bonnet identity of the form (59), but with $\mathcal{R}_{a b c d} \rightarrow{ }^{0} \mathcal{R}_{a b c d}^{\dagger}$. Thus, one can set to zero any one of the parameters $\alpha_{i}$ in (203) with no loss of generality (at least classically). Unlike in 
WGT, however, the Lagrangian (203) still depends on all the gauge fields and their derivatives, in particular the $A$-field. A typical form for the matter Lagrangian density $\mathcal{L}_{M}$ is that given in (192), but with appropriate modifications, ${ }^{94}$ namely,

$$
\mathcal{L}_{\mathrm{M}}=h^{-1}\left[\frac{1}{2} i \bar{\psi} \gamma^{a} 0 \stackrel{\leftrightarrow}{\mathcal{D}}_{a} \psi-\mu \phi \bar{\psi} \psi+\frac{1}{2} v\left({ }^{0} \mathcal{D}_{a}^{\dagger} \phi\right)\left({ }^{0} \mathcal{D}^{\dagger a} \phi\right)-\lambda \phi^{4}-a \phi^{20} \mathcal{R}^{\dagger}\right] .
$$

The total Lagrangian density thus has the following functional dependencies in the most general case:

$$
\mathcal{L}_{\mathrm{T}}=\mathcal{L}_{\mathrm{G}}\left(h, \partial h, \partial^{2} h, A, \partial A, V, \partial V\right)+\mathcal{L}_{\mathrm{M}}\left(\varphi, \partial \varphi, \phi, \partial \phi, h, \partial h, \partial^{2} h, A, \partial A, V, \partial V\right) .
$$

In terms of the alternative set of variables discussed in Section III J, one may equally write

$$
\mathcal{L}_{\mathrm{T}}=\mathcal{L}_{\mathrm{G}}\left(h, \partial h, \partial^{2} h, A^{\dagger}, \partial A^{\dagger}\right)+\mathcal{L}_{\mathrm{M}}\left(\varphi, \partial \varphi, \phi, \partial \phi, h, \partial h, \partial^{2} h, A^{\dagger}, \partial A^{\dagger}\right),
$$

which does not depend explicitly on the $V$ gauge field or its derivatives. In each case, the resulting field equations for the gravitational gauge fields (including the $A$-field equation, which was absent in reduced WGT) will clearly have the same generic structure as those given in Sections III H and III J, respectively, although once again the specific forms for each term are not, in general, obtained from the corresponding "full" eWGT expressions simply by replacing the "full" covariant derivative and field strength tensors with their "reduced" counterparts. For the matter field equations, however, the forms (67a) and (67b) are still valid, but with the replacement $\mathcal{D}_{a}^{\dagger} \rightarrow{ }^{0} \mathcal{D}_{a}^{\dagger}$. As in WGT, however, the gravitational and matter Lagrangians are both, in general, quadratic in second derivatives of the $h$-field (indeed, every term in $\mathcal{L}_{\mathrm{G}}$ and the term proportional to $\phi^{20} \mathcal{R}^{\dagger}$ in $\mathcal{L}_{\mathrm{M}}$ depend on $\partial^{2} h$, whereas in "full" eWGT, only $L_{\mathcal{H}^{\dagger 2}}$ has this dependency). Thus, the resulting field equations will typically be linear in fourth-order derivatives of $h$, and such theories typically suffer from Ostrogradsky's instability, although this needs to be investigated on a case-by-case basis.

The conservation laws in reduced eWGT will have the same form as those given in Section III I, but with the replacements $\mathcal{D}_{a}^{\dagger} \rightarrow{ }^{0} \mathcal{D}_{a}^{\dagger}, \mathcal{R}_{a b c d}^{\dagger} \rightarrow{ }^{0} \mathcal{R}_{a b c d}^{\dagger}$, and $\mathcal{T}_{a b c}^{\dagger} \rightarrow{ }^{0} \mathcal{T}_{a b c}^{\dagger} \equiv 0$. Furthermore, by performing analogous calculations to those presented in Sections III M and III $\mathrm{N}$, respectively, one may show that our conclusions in eWGT regarding the interpretation of the Einstein gauge and the motion of test particles also apply in reduced eWGT.

As was discussed for WGT in Section III O, it is important to distinguish reduced eWGT, in which the condition of vanishing eWGT torsion is imposed directly at the level of the action, from instead setting the (properly eWGT-covariant) condition $\mathcal{T}_{a b c}^{\dagger}=0$ in the field equations of eWGT. In the latter case, aside from terms generated by the term proportional to $\mathcal{H}_{a b}^{\dagger} \mathcal{H}^{\dagger a b}$ in $L_{\mathrm{G}}$ (if included), the basic field equations remain linear in second-order derivatives of the gauge fields. Although one can substitute for the rotational gauge field to obtain terms that contain higher-order derivatives, such theories do not suffer from Ostrogradsky's instability if the term proportional to $\mathcal{H}_{a b}^{\dagger} \mathcal{H}^{\dagger a b}$ is excluded from $L_{\mathrm{G}}$.

\section{P. Geometric interpretation of eWGT}

We have developed eWGT as a gauge theory of gravity in Minkowski spacetime, but one can reinterpret eWGT in geometric terms, along similar lines to WGT, as we presented in Section II O. The central tenet of the geometrical interpretation remains the identification of $h_{a}{ }^{\mu}$ as the components of a vierbein system in a more general spacetime (see Equation (114)). As in WGT, this identification leads to the results (115) and (116) relating the spacetime metric $g_{\mu \nu}$, Lorentz metric $\eta_{a b}$, and the inverse $h$-field $b^{a}{ }_{\mu}$, and consequently the result $h^{-1}=\sqrt{-g}$ also remains valid. Under a (local, physical) extended dilation, the spacetime metric and $h$-field have Weyl weights $w\left(g_{\mu \nu}\right)=2$ and $w\left(h_{a}{ }^{\mu}\right)=-1$, respectively, as in WGT, and so (115) and (116) again imply that $w\left(\eta_{a b}\right)=0$, as expected. As in WGT, one immediately finds that the $h$-field and its inverse are directly related by index raising/lowering, so there is no need to distinguish between them by using different kernel letters. We therefore again notate $h_{a}{ }^{\mu}$ and $b^{b}{ }_{v}$ by $e_{a}{ }^{\mu}$ and $e^{b}{ }_{v}$, respectively.

In eWGT, unlike WGT, it is the combination $A^{\dagger a b}{ }_{\mu}$ in (142) of the three gauge fields $e_{a}{ }^{\mu}$, $A^{a b}{ }_{\mu}$, and $V_{\mu}$ which is naturally interpreted as the components of the spin-connection that encode 
the rotation of the local tetrad frame between points $x$ and $x+\delta x$. As in WGT, this is accompanied by a local change in the standard of length between the two points, but in contrast to WGT this is encoded not only by the dilation gauge field $V_{\mu}$, but also by the trace of the PGT torsion $T_{\mu}$, which depends on $h_{a}{ }^{\mu}$ and $A^{a b}{ }_{\mu}$. Thus, both the rotation of the local tetrad frame and the local change in the standard of length between two points are encoded by all three gauge fields. The operation of parallel transport for some vector $J^{a}$ of weight $w$ is therefore defined as

$$
\delta J^{a}=-\left[A_{b \mu}^{\dagger a}-w\left(V_{\mu}+\frac{1}{3} T_{\mu}\right) \delta_{b}^{a}\right] J^{b} \delta x^{\mu} .
$$

As in WGT, in general, a vector not only changes its direction on parallel transport around a closed loop, but also its length. The expression (207) establishes the correct form for the related $(\Lambda, \rho)$-covariant derivative, e.g.,

$$
\begin{aligned}
D_{\mu}^{\dagger} J^{a} & =\partial_{\mu} J^{a}-w\left(V_{\mu}+\frac{1}{3} T_{\mu}\right) J^{a}+A_{b \mu}^{\dagger a} J^{b} \\
& =\partial_{\mu}^{\dagger} J^{a}+A_{b \mu}^{\dagger a} J^{b},
\end{aligned}
$$

where we have used the partial derivative operator $\partial_{\mu}^{\dagger}$ defined in (146). As in WGT, one can easily deduce that $A_{\mu}^{\dagger a b}=-A_{\mu}^{\dagger b a}$, as previously.

Owing to the necessity of using arbitrary coordinates in the more general spacetime, one must generalise the $(\Lambda, \rho)$-covariant derivative to apply to fields with definite GCT tensor behaviour. Following the approach used in WGT, one defines the "total" covariant derivative

$$
\Delta_{\mu}^{\dagger} \equiv \partial_{\mu}^{\dagger}+\Gamma_{\rho \mu}^{\sigma} \mathrm{X}_{\sigma}^{\rho}+\frac{1}{2} A^{a b}{ }_{\mu} \Sigma_{a b}=\nabla_{\mu}^{\dagger}+D_{\mu}^{\dagger}-\partial_{\mu}^{\dagger},
$$

where $\nabla_{\mu}^{\dagger}=\partial_{\mu}^{\dagger}+\Gamma_{\rho \mu}^{\sigma} \mathrm{X}^{\rho}{ }_{\sigma}$ and $\mathrm{X}^{\rho}{ }_{\sigma}$ are the GL $(4, R)$ generator matrices appropriate to the GCT tensor character of the field to which $\Delta_{\mu}^{\dagger}$ is applied. If a field $\psi$ carries only Latin indices, then $\nabla_{\mu}^{\dagger} \psi=\partial_{\mu}^{\dagger} \psi$ and so $\Delta_{\mu}^{\dagger} \psi=D_{\mu}^{\dagger} \psi$; conversely, if a field $\psi$ carries only Greek indices, then $D_{\mu}^{\dagger} \psi=\partial_{\mu}^{\dagger} \psi$ and so $\Delta_{\mu}^{\dagger} \psi=\nabla_{\mu}^{\dagger} \psi$. When acting on an object of weight $w$, for all these derivative operators the resulting object also transforms covariantly with the same weight $w$.

The affine connection coefficients $\Gamma_{\rho \mu}^{\sigma}$ again become dynamical variables, but are necessarily related to the spin-connection and dilation current, since (120) should still hold provided $J^{a}$ has weight $w=1$. This result then yields the relation

$$
\Delta_{\mu}^{\dagger} e^{a}{ }_{v} \equiv \partial_{\mu}^{\dagger} e^{a}{ }_{v}-\Gamma^{\sigma}{ }_{v \mu} e^{a}{ }_{\sigma}+A^{\dagger a}{ }_{b \mu} e^{b}{ }_{v}=0
$$

which relates $A$ and $\Gamma$ (and $V$ and $T$ ). Comparing this result with the equivalent relation (121) in WGT, we see that (210) is obtained simply by making the replacements $\partial_{\mu}^{*} \rightarrow \partial_{\mu}^{\dagger}$ and $A_{\mu}^{a b} \rightarrow A_{\mu}^{\dagger a b}$. Consequently, the results (122) and (123) explicitly relating $A$ and $\Gamma$ are simply replaced by expressions of the same form but with the above substitutions. As in WGT, this replacement strategy enables one to straightforwardly derive the further relationships in the geometric interpretation of eWGT that correspond to the results (124)-(132) in WGT.

In particular, using (115) and (210), one finds that $\nabla_{\sigma}^{\dagger} g_{\mu \nu}=0$, and so this derivative operator commutes with raising and lowering of coordinate indices. Equivalently, one may write this as the semi-metricity condition

$$
\nabla_{\sigma} g_{\mu \nu}=2\left(V_{\sigma}+\frac{1}{3} T_{\sigma}\right) g_{\mu \nu}
$$

Comparing this result with the corresponding semi-metricity condition (124) in WGT, we see that it has a similar form. ${ }^{95}$ In addition to depending on the dilation gauge field $V_{\mu}$, however, the eWGT version also depends on the trace of the PGT torsion, which is itself a function of $e_{a}{ }^{\mu}$ and $A^{a b}{ }_{\mu}$. Therefore, the spacetime may be considered as having some extended form of Weyl-Cartan geometry. To our knowledge, spacetimes with the particular semi-metricity condition (211) have not been studied previously; in what follows, we refer to them as $\widehat{Y}_{4}$ spacetimes. Moreover, one finds that

$$
\begin{aligned}
R_{\sigma \mu \nu}^{\dagger \rho_{\sigma \nu}} & =2\left(\partial_{[\mu} \Gamma_{|\sigma| v]}^{\rho}+\Gamma_{\lambda[\mu}^{\rho} \Gamma_{|\sigma| v]}^{\lambda}\right)+H_{\mu \nu}^{\dagger} \delta_{\sigma \sigma}^{\rho}, \\
T_{\mu \nu}^{\dagger \lambda} & =2 \Gamma_{[\nu \mu]}^{\lambda}, \\
H_{\mu \nu}^{\dagger} & =\partial_{\mu}\left(V_{\nu}+\frac{1}{3} T_{\nu}\right)-\partial_{\nu}\left(V_{\mu}+\frac{1}{3} T_{\mu}\right),
\end{aligned}
$$


where $R^{\dagger \rho}{ }_{\sigma \mu \nu}=e_{a}{ }^{\rho} e^{b}{ }_{\sigma} R^{\dagger a}{ }_{b \mu \nu}$ and $T^{\dagger \lambda}{ }_{\mu \nu}=e_{a}^{\lambda} T^{\dagger a}{ }_{\mu \nu}$. We thus recognise (213) as (minus) the torsion tensor of the $\widehat{Y}_{4}$ spacetime. One further finds that the trace of the torsion vanishes, $T^{\dagger \lambda}{ }_{\mu \lambda}=0$, so that the affine connection has the additional symmetry property

$$
\Gamma_{\mu \lambda}^{\lambda}=\Gamma_{\lambda \mu}^{\lambda}
$$

From (212), we see that $R^{\dagger} \rho_{\sigma \mu \nu}$ is not simply its Riemann tensor. Rather, the Riemann tensor of the $\widehat{Y}_{4}$ spacetime is given by

$$
\widehat{R}_{\sigma \mu \nu}^{\rho} \equiv R_{\sigma \mu \nu}^{\dagger \rho}-H_{\mu \nu}^{\dagger} \delta_{\sigma}^{\rho} .
$$

One should note that, although $\widehat{R}_{\rho \sigma \mu \nu}$ is antisymmetric in $(\mu, v)$, it is not antisymmetric in $(\rho, \sigma)$, since $\widehat{R}_{(\rho \sigma) \mu \nu}=-g_{\rho \sigma} H_{\mu \nu}^{\dagger}$. Indeed, it does not satisfy the usual cyclic and Bianchi identities of the Riemann tensor in a Riemannian $V_{4}$ spacetime. One may also show that, with the given arrangements of indices, both $\widehat{R}_{\sigma \mu \nu}^{\rho}$ (or $R^{\dagger \rho}{ }_{\sigma \nu \nu}$ ) and $T^{\dagger \lambda}{ }_{\mu \nu}$ transform covariantly with weight $w=0$ under a local dilation. It is also worth noting that $\widehat{R}_{\mu \nu} \equiv \widehat{R}_{\mu \lambda \nu}{ }^{\lambda}=R_{\mu \nu}^{\dagger}+H_{\mu \nu}^{\dagger}$ and $\widehat{R} \equiv \widehat{R}_{\mu}^{\mu}=R^{\dagger}$. In a similar manner to WGT, the quantities (212)-(216) arise naturally in the expression for the commutator of two derivative operators acting on a vector $J^{\rho}$ (say) of Weyl weight $w$, which is given by

$$
\left[\nabla_{\mu}^{\dagger}, \nabla_{\nu}^{\dagger}\right] J^{\rho}=\widehat{R}_{\sigma \mu \nu}^{\rho} J^{\sigma}-w H_{\mu \nu}^{\dagger} J^{\rho}-T_{\mu \nu}^{\dagger \sigma} \nabla_{\sigma}^{\dagger} V^{\rho} .
$$

One also finds that the affine connection must satisfy

$$
\Gamma_{\mu \nu}^{\lambda}={ }^{0} \Gamma_{\mu \nu}^{\dagger \lambda}+K_{\mu \nu}^{\dagger \lambda},
$$

where the first term on the RHS reads

$$
\begin{aligned}
{ }^{0} \Gamma_{\mu \nu}^{\dagger \lambda} & =\frac{1}{2} g^{\lambda \rho}\left(\partial_{\mu}^{\dagger} g_{\nu \rho}+\partial_{\nu}^{\dagger} g_{\mu \rho}-\partial_{\rho}^{\dagger} g_{\mu \nu}\right) \\
& ={ }^{0} \Gamma_{\mu \nu}^{\lambda}-\delta_{v}^{\lambda}\left(V_{\mu}+\frac{1}{3} T_{\mu}\right)-\delta_{\mu}^{\lambda}\left(V_{v}+\frac{1}{3} T_{\nu}\right)+g_{\mu \nu}\left(V^{\lambda}+\frac{1}{3} T^{\lambda}\right),
\end{aligned}
$$

in which ${ }^{0} \Gamma^{\lambda}{ }_{\mu \nu}$ is the standard metric (Christoffel) connection and $K^{\dagger \lambda}{ }_{\mu \nu}$ is the $\widehat{Y}_{4}$ contortion tensor,

$$
K_{\mu \nu}^{\dagger \lambda}=-\frac{1}{2}\left(T_{\mu \nu}^{\dagger \lambda}-T^{\dagger}{ }_{\nu}^{\lambda}{ }_{\mu}+T_{\mu \nu}^{\dagger}{ }^{\lambda}\right) \text {. }
$$

In direct comparison with our treatment of WGT, the result (218) is the analogue of the expression (158) in the gauge theory viewpoint. We may consider the geometric interpretation of the quantities ${ }^{0} A^{\dagger a b}{ }_{\mu}$, introduced in (158), but note that they depend on all the gauge fields; this contrasts with PGT and WGT, for which the corresponding quantities do not depend on the $A$-field. Following an analogous argument to that given above, but considering instead the reduced covariant derivative ${ }^{0} D_{\mu}^{\dagger}$, as defined in (159), one finds that ${ }^{0} A^{\dagger a b}{ }_{\mu}$ and the connection ${ }^{0} \Gamma^{\dagger \sigma}{ }_{\rho \mu}$ represent the same geometrical object in two different frames, and one obtains a "reduced" form of the tetrad postulate (210) given by

$$
{ }^{0} \Delta_{\mu}^{\dagger} e^{a}{ }_{\nu} \equiv \partial_{\mu}^{\dagger} e^{a}{ }_{\nu}-{ }^{0} \Gamma^{\dagger \sigma}{ }_{v \mu} e^{a}{ }_{\sigma}+{ }^{0} A^{\dagger a}{ }_{b \mu} e^{b}{ }_{\nu}=0
$$

It thus follows that the relationships (122) and (123) again hold with the replacements $\partial_{\mu}^{*} \rightarrow \partial_{\mu}^{\dagger}$, $\Gamma^{\sigma}{ }_{v \mu} \rightarrow{ }^{0} \Gamma^{\dagger \sigma}{ }_{v \mu}$, and $A^{a}{ }_{b \mu} \rightarrow{ }^{0} A^{\dagger a}{ }_{b \mu}$, from which one can directly derive (219). One also obtains the metricity condition ${ }^{0} \nabla_{\sigma}^{\dagger} g_{\mu \nu}=0$. Finally, the expression (212) for the curvature also holds, but with $R^{\dagger \rho}{ }_{\sigma \mu \nu} \rightarrow{ }^{0} R^{\dagger \rho} \sigma_{\sigma \mu \nu}$ and $\Gamma^{\sigma}{ }_{\nu \mu} \rightarrow{ }^{0} \Gamma^{\dagger \sigma}{ }_{\nu \mu}$, whereas (213) becomes simply ${ }^{0} T^{\dagger \lambda}{ }_{\mu \nu}=0$, indicating the absence of torsion, as expected. The expression (217) is also valid, but with $\nabla_{\mu}^{\dagger} \rightarrow{ }^{0} \nabla_{\mu}^{\dagger}$, $R_{\sigma \mu \nu}^{\rho} \rightarrow{ }^{0} R^{\dagger \rho} \rho_{\sigma \mu \nu}$, and $T^{\dagger \sigma}{ }_{\mu \nu} \rightarrow{ }^{0} T^{\dagger \sigma}{ }_{\mu \nu}=0$.

\section{QUADRATIC EXTENDED WEYL GAUGE THEORY OF GRAVITY}

The new scale-invariant gauge theory of gravity that we now consider in detail has a total Lagrangian $L_{\mathrm{T}}=L_{\mathrm{G}}+L_{\mathrm{M}}$ corresponding to the general eWGT form (167). 
In the free gravitational sector, it is defined by the most general parity-invariant ${ }^{96}$ eWGT Lagrangian (166) that is at most quadratic in the eWGT field strengths, namely, $L_{G}=L_{\mathcal{R}^{\dagger 2}}+L_{\mathcal{H}^{\dagger 2}}$, where

$$
\begin{aligned}
L_{\mathcal{R}^{\dagger 2}}= & \alpha_{1} \mathcal{R}^{\dagger 2}+\alpha_{2} \mathcal{R}_{a b}^{\dagger} \mathcal{R}^{\dagger a b}+\alpha_{3} \mathcal{R}_{a b}^{\dagger} \mathcal{R}^{\dagger b a}+\alpha_{4} \mathcal{R}_{a b c d}^{\dagger} \mathcal{R}^{\dagger a b c d}+\alpha_{5} \mathcal{R}_{a b c d}^{\dagger} \mathcal{R}^{\dagger a c b d} \\
& +\alpha_{6} \mathcal{R}_{a b c d}^{\dagger} \mathcal{R}^{\dagger c d a b}, \\
L_{\mathcal{H}^{\dagger 2}}= & \frac{1}{2} \xi \mathcal{H}_{a b}^{\dagger} \mathcal{H}^{\dagger a b},
\end{aligned}
$$

in which $\alpha_{i}$ and $\xi$ are dimensionless parameters and the factor of $\frac{1}{2}$ has been introduced for later convenience. As discussed in Section III G, the eWGT field strength $\mathcal{R}_{a b c d}^{\dagger}$ satisfies a form of the Gauss-Bonnet identity such that, with no loss of generality (up to a classically unimportant boundary term in $D \leq 4$ dimensions), one is free to set one of $\alpha_{1}, \alpha_{3}$, or $\alpha_{6}$ equal to zero. In what follows, we will retain all these terms, but this freedom should be borne in mind during our analysis. ${ }^{97}$

In the matter sector, we base our Lagrangian on (192), which builds on Dirac's original suggestion for accommodating "ordinary" matter in scale-invariant theories of gravity. In particular, we adopt the form $L_{\mathrm{M}}=L_{\varphi}+L_{\phi}+\phi^{2} L_{\mathcal{R}^{\dagger}}+\phi^{2} L_{\mathcal{T}^{\dagger 2}}$, where

$$
\begin{aligned}
L_{\varphi} & =L_{\varphi}(\varphi, \partial \varphi, h, \partial h, A, V, \phi), \\
L_{\phi} & =\frac{1}{2} \nu \mathcal{D}_{a}^{\dagger} \phi \mathcal{D}^{\dagger a} \phi-\lambda \phi^{4}, \\
L_{\mathcal{R}^{\dagger}} & =-\frac{1}{2} a \mathcal{R}^{\dagger}, \\
L_{\mathcal{T}^{\dagger 2}} & =\beta_{1} \mathcal{T}_{a b c}^{\dagger} \mathcal{T}^{\dagger a b c}+\beta_{2} \mathcal{T}_{a b c}^{\dagger} \mathcal{T}^{\dagger b a c},
\end{aligned}
$$

in which $v, \lambda, a$, and $\beta_{i}$ are again dimensionless parameters (with $v, \lambda$, and $a$ usually positive) and some factors of $\frac{1}{2}$ have been introduced for later convenience. ${ }^{98}$ For the moment we allow the form of $L_{\varphi}$ for the matter field $\varphi$ to remain general and possibly include a dependence on the scalar field $\phi$.

In this section, we limit ourselves to deriving the field equations for the above theory and commenting briefly on their general structure. The phenomenological content of these field equations, including their application and solution in various astrophysical and cosmological situations, will be discussed in forthcoming papers.

\section{A. Field equations}

Recalling the discussion of Section III H, the theory has only three independent field equations, namely, the $h$-equation (171), the $A$-equation (168b), and the $\varphi$-equation (172a). We calculate the explicit forms of the first two of these equations (recall we are leaving $L_{\varphi}$ general for the moment), without setting to zero any of the dimensionless parameters in (222)-(227). This entails a very long, but nevertheless straightforward, calculation, the results of which are given below. In addition, although they are not independent equations of motion, we will also calculate the $V$-field equation (168c) and the $\phi$-field equation (172b) to check that they are indeed related simply to the contracted $A$-equation and the contracted $h$-equation, respectively, through the results (177), (178d), (185), and (186). This provides a useful, albeit partial, check on our calculations.

\section{The $h$-field equation}

The $h$-field equation (171) takes the form

$$
\left(t_{\mathcal{R}^{2}}^{\dagger}\right)^{a}{ }_{b}+\left(t_{\mathcal{H}^{2}}^{\dagger}\right)^{a}{ }_{b}+\left(\tau_{\varphi}^{\dagger}\right)^{a}{ }_{b}+\left(\tau_{\phi}^{\dagger}\right)^{a}{ }_{b}+\left(\tau_{\mathcal{R}}^{\dagger}\right)_{b}{ }_{b}+\left(\tau_{\mathcal{T}^{2}}^{\dagger}\right)^{a}{ }_{b}=0,
$$

where, in the free gravitational sector, one finds

$$
\begin{aligned}
h\left(t_{\mathcal{R}^{2}}^{\dagger}{ }^{a}{ }_{b}=\right. & \alpha_{1} \mathcal{R}^{\dagger}\left(4 \mathcal{R}^{\dagger a}{ }_{b}-\delta_{b}^{a} \mathcal{R}^{\dagger}\right) \\
& +\alpha_{2}\left[2\left(\mathcal{R}^{\dagger c a} \mathcal{R}_{c b}^{\dagger}-\mathcal{R}^{\dagger c d} \mathcal{R}^{\dagger a}{ }_{c d b}\right)-\delta_{b}^{a} \mathcal{R}_{c d}^{\dagger} \mathcal{R}^{\dagger c d}\right] \\
& +\alpha_{3}\left[2\left(\mathcal{R}^{\dagger a c} \mathcal{R}_{c b}^{\dagger}-\mathcal{R}^{\dagger d c} \mathcal{R}^{\dagger a}{ }_{c d b}\right)-\delta_{b}^{a} \mathcal{R}_{c d}^{\dagger} \mathcal{R}^{\dagger d c}\right]
\end{aligned}
$$




$$
\begin{aligned}
& +\alpha_{4}\left[4 \mathcal{R}^{\dagger c d e a} \mathcal{R}_{c d e b}^{\dagger}-\delta_{b}^{a} \mathcal{R}^{\dagger c d e f} \mathcal{R}_{c d e f}^{\dagger}\right] \\
& +\alpha_{5}\left[2\left(\mathcal{R}^{\dagger a c d e}-\mathcal{R}^{\dagger e c d a}\right) \mathcal{R}_{c d e b}^{\dagger}-\delta_{b}^{a} \mathcal{R}^{\dagger c e d f} \mathcal{R}_{c d e f}^{\dagger}\right] \\
& +\alpha_{6}\left[4 \mathcal{R}^{\dagger e a c d} \mathcal{R}_{c d e b}^{\dagger}-\delta_{b}^{a} \mathcal{R}^{\dagger e f c d} \mathcal{R}_{c d e f}^{\dagger}\right], \\
h\left(t_{\mathcal{H}^{2}}^{\dagger}{ }^{a}{ }_{b}{ }_{b}=\right. & \frac{1}{2} \xi\left[4 \mathcal{H}_{b c}^{\dagger} \mathcal{H}^{\dagger a c}-\delta_{b}^{a} \mathcal{H}_{c d}^{\dagger} \mathcal{H}^{\dagger c d}+\frac{4}{3} \mathcal{D}_{b}^{\dagger}\left(\mathcal{D}_{c}^{\dagger} \mathcal{H}^{\dagger c a}-\frac{1}{2} \mathcal{T}^{\dagger a}{ }_{c d} \mathcal{H}^{\dagger c d}\right)\right],
\end{aligned}
$$

and in the matter sector, in addition to $\left(\tau_{\varphi}^{\dagger}\right)^{a}{ }_{b}=h_{b}{ }^{\mu}\left(\delta L_{\varphi} / \delta h_{a}{ }^{\mu}\right)_{\dagger}$, one has

$$
\begin{aligned}
h\left(\tau_{\phi}^{\dagger}\right)^{a}{ }_{b}= & \frac{1}{2} v\left[\frac{4}{3} \mathcal{D}^{\dagger a} \phi \mathcal{D}_{b}^{\dagger} \phi-\frac{1}{3} \delta_{b}^{a} \mathcal{D}^{\dagger c} \phi \mathcal{D}_{c}^{\dagger} \phi+\frac{2}{3} \phi\left(\delta_{b}^{a} \mathcal{D}_{c}^{\dagger} \mathcal{D}^{\dagger c} \phi-\mathcal{D}_{b}^{\dagger} \mathcal{D}^{\dagger a} \phi\right)\right]+\lambda \delta_{b}^{a} \phi^{4}, \\
h\left(\tau_{\mathcal{R}}^{\dagger}\right)_{b}{ }_{b}= & -a \phi^{2}\left(\mathcal{R}^{\dagger a}{ }_{b}-\frac{1}{2} \delta_{b}^{a} \mathcal{R}^{\dagger}\right), \\
h\left(\tau_{\mathcal{T}^{2}}^{\dagger}\right)^{a}{ }_{b}= & \beta_{1}\left[\phi^{2}\left(4 \mathcal{T}^{\dagger c d a} \mathcal{T}_{c d b}^{\dagger}-2 \mathcal{T}^{\dagger a d e} \mathcal{T}_{b d e}^{\dagger}-\delta_{b}^{a} \mathcal{T}^{\dagger c d e} \mathcal{T}_{c d e}^{\dagger}\right)+4 \mathcal{D}_{c}^{\dagger}\left(\phi^{2} \mathcal{T}_{b}^{\dagger c a}\right)\right] \\
& \left.+\beta_{2}\left[\phi^{2}\left(2 \mathcal{T}^{\dagger d c a} \mathcal{T}_{c d b}^{\dagger}+2 \mathcal{T}^{\dagger a d c} \mathcal{T}_{c d b}^{\dagger}-2 \mathcal{T}^{\dagger a c d} \mathcal{T}_{c b d}^{\dagger}-\delta_{b}^{a} \mathcal{T}^{\dagger d c e} \mathcal{T}_{c d e}^{\dagger}\right)+4 \mathcal{D}_{c}^{\dagger}\left(\phi^{2} \mathcal{T}^{\dagger[c}{ }_{b}{ }^{a}\right]\right)\right] .
\end{aligned}
$$

In particular, we note that $\left(t_{\mathcal{H}^{2}}^{\dagger}\right)^{a}{ }_{b}$ in (230) is linear in fourth-order derivatives of the $h$-field. As discussed in Section III H, this occurs because the dilation field strength $\mathcal{H}_{a b}^{\dagger}$ is itself linear in the second-order derivatives of the $h$-field. Moreover, since $\mathcal{H}_{a b}^{\dagger}$ contains first-order derivatives of the $h, A$, and $V$ gauge fields, $\left(t_{\mathcal{H}^{2}}^{\dagger}\right)_{b}^{a}$ also depends on third-order derivatives of these fields. We will shortly discuss this issue in detail in Section IV B.

\section{The A-field equation}

The $A$-field equation (168b) has the form

$$
\left(s_{\mathcal{R}^{2}}\right)_{a b}{ }^{c}+\left(s_{\mathcal{H}^{2}}\right)_{a b}{ }^{c}+\left(\sigma_{\varphi}\right)_{a b}{ }^{c}+\left(\sigma_{\phi}\right)_{a b}{ }^{c}+\left(\sigma_{\mathcal{R}}\right)_{a b}{ }^{c}+\left(\sigma_{\mathcal{T}^{2}}\right)_{a b}{ }^{c}=0,
$$

where, in the free gravitational sector,

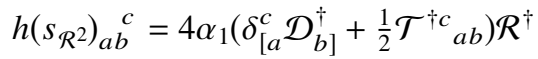

$$
\begin{aligned}
& +4 \alpha_{2}\left(\delta_{\left[d^{c}\right.}^{c} \mathcal{D}_{e]}^{\dagger}+\frac{1}{2} \mathcal{T}^{\dagger c}{ }_{d e}\right) \mathcal{R}_{[a}^{\dagger d} \delta_{b]}^{e} \\
& +4 \alpha_{3}\left(\delta_{\left[d^{c}\right.}^{c} \mathcal{D}_{e]}^{\dagger}+\frac{1}{2} \mathcal{T}^{\dagger c}{ }_{d e}\right) \mathcal{R}^{\dagger{ }_{[a}} \delta_{b]}^{e} \\
& +4 \alpha_{4}\left(\delta_{\left[d^{c}\right.}^{c} \mathcal{D}_{e]}^{\dagger}+\frac{1}{2} \mathcal{T}^{\dagger c}{ }_{d e}\right) \mathcal{R}_{a b}^{\dagger d e} \\
& \left.+4 \alpha_{5}\left(\delta_{\left[d^{c}\right.}^{c} \mathcal{D}_{e]}^{\dagger}+\frac{1}{2} \mathcal{T}^{\dagger c}{ }_{d e}\right) \mathcal{R}_{[a b]}^{\dagger\left[d_{[a b}\right.} e\right] \\
& +4 \alpha_{6}\left(\delta_{\left[d^{c}\right.}^{c} \mathcal{D}_{e]}^{\dagger}+\frac{1}{2} \mathcal{T}^{\dagger c}{ }_{d e}\right) \mathcal{R}^{\dagger d e}{ }_{a b}, \\
& h\left(s_{\mathcal{H}^{2}}\right)_{a b}{ }^{c}=\frac{2}{3} \xi \eta_{f[b} \delta_{a]}^{c}\left(\mathcal{D}_{e}^{\dagger} \mathcal{H}^{\dagger e f}-\frac{1}{2} \mathcal{T}^{\dagger f}{ }_{d e} \mathcal{H}^{\dagger d e}\right),
\end{aligned}
$$

and in the matter sector, in addition to $\left(\sigma_{\varphi}^{\dagger}\right)_{a b}{ }^{c}=b^{c}{ }_{\mu} \delta L_{\varphi} / \delta A^{a b}{ }_{\mu}$, one has

$$
\begin{aligned}
h\left(\sigma_{\phi}\right)_{a b}{ }^{c} & =-\frac{1}{3} \nu \phi \delta_{[a}^{c} \mathcal{D}_{b]}^{\dagger} \phi, \\
h\left(\sigma_{\mathcal{R}}\right)_{a b}{ }^{c} & =-a\left(\delta_{[a}^{c} \mathcal{D}_{b]}^{\dagger}+\frac{1}{2} \mathcal{T}^{\dagger c}{ }_{a b}\right) \phi^{2}, \\
h\left(\sigma_{\mathcal{T}^{2}}\right)_{a b}{ }^{c} & =-4 \beta_{1} \phi^{2} \mathcal{T}^{\dagger}{ }_{[a b]}{ }^{c}+2 \beta_{2} \phi^{2}\left(\mathcal{T}^{\dagger c}{ }_{a b}+\mathcal{T}^{\dagger}{ }_{[a b]}{ }^{c}\right) .
\end{aligned}
$$

We note that $\left(s_{\mathcal{H}^{2}}\right)_{a b}{ }^{c}$ in (236) is linear in third-order derivatives of the $h$-field but, as mentioned above, we will shortly discuss this issue in detail in Section IV B. It is also worth noting in (239) that the translational field strength satisfies the identity $\mathcal{T}_{c a b}^{\dagger}+2 \mathcal{T}_{[a b] c}^{\dagger}-3 \mathcal{T}_{[c a b]}^{\dagger}=0$.

As one might expect, the contributions to the $A$-field equation (234) arising from the Riemannsquared terms in the action (namely, those in (235) proportional to $\alpha_{4}, \alpha_{5}$, and $\alpha_{6}$, respectively) have a form similar to the LHS of the equation of motion (194) for the electromagnetic field. Indeed, recalling that $\mathcal{R}_{a b c d}^{\dagger}$ is antisymmetric in $a$ and $b$, the term proportional to $\alpha_{4}$ in (236) is 
completely analogous to the LHS of (194). Moreover, the term proportional to $\alpha_{6}$ in (235) can be brought into the same form by working in terms of the adjoint Riemann tensor $\overline{\mathcal{R}}_{a b c d}^{\dagger} \equiv \mathcal{R}_{c d a b}^{\dagger}$. Since $\overline{\mathcal{R}}^{\dagger a}{ }_{b}=\mathcal{R}_{b}^{\dagger}$, one also sees that terms in (235) proportional to $\alpha_{2}$ and $\alpha_{3}$, respectively, may be written in a symmetrical fashion in terms of the Ricci tensor and its adjoint; this is also true for the term proportional to $\alpha_{1}$, since $\overline{\mathcal{R}}^{\dagger}=\mathcal{R}^{\dagger}$. The term proportional to $\alpha_{5}$, however, cannot be written in a simpler or more symmetric form using adjoints. Nonetheless, this is to be expected, since only the term proportional to $\alpha_{5}$ in the Lagrangian (222) cannot be written as the product of the Riemann, or one of its contractions, and the corresponding adjoint quantity. Finally, we note also that the Bianchi identity (161a) satisfied by the Riemann tensor (and its adjoint) is analogous to the identity (195) satisfied by the EM field strength tensor.

Similar comments to the above apply to the contribution (236) to the $A$-field equation, which contains a factor identical in form to the LHS of EM field equation (194), but in terms of the dilation field strength tensor $\mathcal{H}_{a b}^{\dagger}$. Moreover, $\mathcal{H}_{a b}^{\dagger}$ satisfies the Bianchi identity (161c), which is again directly analogous to the identity (195) satisfied by the EM field strength tensor. The above observations taken together suggest that one might search for solutions of the $A$-field equation (234) using techniques derived from electromagnetism. This is indeed the case and will be discussed in a forthcoming paper.

\section{The $V$-field equation}

The $V$-field equation (168c) has the form

$$
\left(j_{\mathcal{R}^{2}}\right)_{a}+\left(j_{\mathcal{H}^{2}}\right)_{a}+\left(\zeta_{\varphi}\right)_{a}+\left(\zeta_{\phi}\right)_{a}+\left(\zeta_{\mathcal{R}}\right)_{a}+\left(\zeta_{\mathcal{T}^{2}}\right)_{a}=0
$$

where, in the free gravitational sector,

$$
\begin{aligned}
h\left(j_{\left.\mathcal{R}^{2}\right)_{a}=}\right. & -12 \alpha_{1} \mathcal{D}_{a}^{\dagger} \mathcal{R}^{\dagger} \\
& -4 \alpha_{2}\left[\mathcal{D}_{b}^{\dagger}\left(\mathcal{R}_{a}^{\dagger b}+\frac{1}{2} \delta_{a}^{b} \mathcal{R}^{\dagger}\right)-\frac{1}{2} \mathcal{T}^{\dagger b}{ }_{a c} \mathcal{R}_{b}^{\dagger c}\right] \\
& -4 \alpha_{3}\left[\mathcal{D}_{b}^{\dagger}\left(\mathcal{R}^{\dagger b}{ }_{a}+\frac{1}{2} \delta_{a}^{b} \mathcal{R}^{\dagger}\right)-\frac{1}{2} \mathcal{T}^{\dagger b}{ }_{a c} \mathcal{R}^{\dagger c}{ }_{b}\right] \\
& -8 \alpha_{4}\left[\mathcal{D}_{b}^{\dagger} \mathcal{R}_{a}^{\dagger b}-\frac{1}{2} \mathcal{T}^{\dagger b c d} \mathcal{R}_{a b c d}^{\dagger}\right] \\
& -2 \alpha_{5}\left[\mathcal{D}_{b}^{\dagger}\left(\mathcal{R}_{a}^{\dagger b}+\mathcal{R}^{\dagger b}{ }_{a}\right)-\mathcal{T}^{\dagger b c d}\left(\mathcal{R}_{c b a d}^{\dagger}+\mathcal{R}_{c a b d}^{\dagger}\right)\right] \\
& -8 \alpha_{6}\left[\mathcal{D}_{b}^{\dagger} \mathcal{R}^{\dagger b}{ }_{a}-\frac{1}{2} \mathcal{T}^{\dagger b c d} \mathcal{R}_{c d a b}^{\dagger}\right], \\
h\left(j_{\mathcal{H}^{2}}\right)_{a}= & -2 \xi\left[\mathcal{D}_{b}^{\dagger} \mathcal{H}^{\dagger b}{ }_{a}-\frac{1}{2} \mathcal{T}^{\dagger}{ }_{a c d} \mathcal{H}^{\dagger c d}\right],
\end{aligned}
$$

and in the matter sector, in addition to $\left(\zeta_{\varphi}\right)_{a}=b_{a \mu} \delta L_{\varphi} / \delta V_{\mu}$,

$$
\begin{aligned}
h\left(\zeta_{\phi}\right)_{a} & =v \phi \mathcal{D}_{a}^{\dagger} \phi, \\
h\left(\zeta_{\mathcal{R}}\right)_{a} & =3 a \mathcal{D}_{a}^{\dagger} \phi^{2}, \\
h\left(\zeta_{\mathcal{T}^{2}}\right)_{a} & =0 .
\end{aligned}
$$

By comparing the expressions (241)-(245) with those given in (235)-(239), we see that, as anticipated, the $V$-equation (240) is indeed identical to (twice) the $A$-equation (234) contracted on the indices $b$ and $c$, in accordance with the general results (185) and (186), and so $j_{a}^{\dagger}$ and $\zeta_{a}^{\dagger}$ vanish identically for each part of the Lagrangian.

\section{The $\phi$-field equation}

Finally, the $\phi$-field equation (172b) has the form

$$
\partial_{\phi} L_{\mathcal{R}^{2}}+\partial_{\phi} L_{\mathcal{H}^{2}}+\partial_{\phi} L_{\varphi}+\delta_{\phi} L_{\phi}+\partial_{\phi} L_{\mathcal{R}}+\partial_{\phi} L_{\mathcal{T}^{2}}=0
$$


where $\partial_{\phi} \equiv \partial / \partial \phi$ and $\delta_{\phi} \equiv \delta / \delta \phi$, since only $L_{\phi}$ depends on the derivatives of $\phi$. In the free gravitational sector,

$$
\begin{aligned}
\partial_{\phi} L_{\mathcal{R}^{2}} & =0, \\
\partial_{\phi} L_{\mathcal{H}^{2}} & =0,
\end{aligned}
$$

and, in the matter sector,

$$
\begin{aligned}
\delta_{\phi} L_{\phi} & =-v \mathcal{D}_{a}^{\dagger}\left(\mathcal{D}^{\dagger a} \phi\right)-4 \lambda \phi^{3}, \\
\partial_{\phi} L_{\mathcal{R}} & =-a \phi^{2} \mathcal{R}^{\dagger}, \\
\partial_{\phi} L_{\mathcal{T}^{2}} & =2 \beta_{1} \phi \mathcal{T}_{a b c}^{\dagger} \mathcal{T}^{\dagger a b c}+2 \beta_{2} \phi \mathcal{T}_{a b c}^{\dagger} \mathcal{T}^{\dagger b a c} .
\end{aligned}
$$

By comparing the expressions (247)-(251) with those given in (229)-(233), we see that, as anticipated, the $\phi$-equation (246) is indeed identical to the $h$-equation (228) contracted on the indices $a$ and $b$ and divided through by $-\phi$, in accordance with the general results (177) and (178d), assuming the $\varphi$-equation $\delta_{\varphi} L_{\varphi}=0$ is satisfied.

Since the number of terms in the $\phi$-equation is quite small, it is worth writing it out as a single equation, which reads

$$
v \mathcal{D}_{a}^{\dagger}\left(\mathcal{D}^{\dagger a} \phi\right)+4 \lambda \phi^{3}-\partial_{\phi} L_{\varphi}+\phi\left(a \mathcal{R}^{\dagger}-2 \beta_{1} \mathcal{T}_{a b c}^{\dagger} \mathcal{T}^{\dagger a b c}-2 \beta_{2} \mathcal{T}_{a b c}^{\dagger} \mathcal{T}^{\dagger b a c}\right)=0 .
$$

\section{B. Higher derivative terms in the field equations}

As we have mentioned several times, and demonstrated above, if one includes in the free gravitational Lagrangian the term proportional to $\mathcal{H}_{a b}^{\dagger} \mathcal{H}^{\dagger a b}$, namely, (223), the corresponding terms it generates in the $h$-field equation are linear in fourth-order derivatives of the $h$-field and also contain third-order derivatives of all three gauge fields $h, A$, and $V$. Moreover, the terms generated in the $A$-field equation are linear in third-order derivatives of the $h$-field (we need not consider the field equations for $\phi$ and $V$, since they are related simply to contractions of the $h$ and $A$ field equations, respectively).

At first sight, this would seem to indicate that theories containing the $\mathcal{H}_{a b}^{\dagger} \mathcal{H}^{\dagger a b}$ term in the Lagrangian suffer from Ostrogradsky's instability. This conclusion is not clear cut, however, since in applying such theories to particular physical systems, which we will discuss in forthcoming papers, we have found in every case that the field equations organise themselves into combinations of coupled second-order equations in the gauge fields. Specifically, one finds the terms containing third- or fourth-order derivatives correspond to the derivative of already known expressions and so contain no new information. Moreover, on linearising the set of field equations displayed above, which we will also discuss in a forthcoming publication, we find that the same behaviour occurs generally, independent of any application of the theory to a particular physical system. Since the leading order of the field equations is preserved by linearisation, this suggests that the full non-linear set of field equations derived above should also enjoy this general property and hence not suffer from Ostrogradsky's instability, although we do not yet have a direct proof in this case. Consequently, we have chosen to retain the terms resulting from the inclusion of the $\mathcal{H}_{a b}^{\dagger} \mathcal{H}^{\dagger a b}$ in the field equations given above, so that they are readily available if required.

The obvious alternative approach to ensuring that Ostrogradsky's instability does not occur is simply to omit the term proportional to $\mathcal{H}_{a b}^{\dagger} \mathcal{H}^{\dagger a b}$ from the free gravitational Lagrangian (by setting $\xi=0$ ). Indeed, one can argue that the inclusion of the $\mathcal{H}_{a b}^{\dagger} \mathcal{H}^{\dagger a b}$ term as the kinetic term for the $V$ gauge field is not well motivated. As discussed in Section III J, eWGTs of the general form considered here can be written entirely in terms of the two combinations $h$ and $A^{\dagger}$ of the gauge fields, such that there is no explicit dependence on the dilation gauge field $V$ or its derivatives. Moreover, the natural field strength tensors for the $h$ and $A^{\dagger}$ fields are $\mathcal{T}_{a b c}^{\dagger}$ and $\mathcal{R}_{a b c d}^{\dagger}$, respectively, and the total Lagrangian already contains all possible (parity-invariant) terms quadratic in these field strengths without including the $\mathcal{H}_{a b}^{\dagger} \mathcal{H}^{\dagger a b}$ term. The field equations in the special case $\xi=0$ still display a rich phenomenology, and there is already a good deal to be explored in them. In particular, we note that in this case the linearisation process mentioned above reveals the further desirable property that 
one can obtain a second-order equation in $V$ alone, which can then be used to solve for $A$ alone and thence for the $h$ function alone, with sensible boundary conditions being available at each stage. Thus, in this case, the theory is inherently fully solvable. These issues will be discussed fully in a forthcoming paper.

\section{Einstein gauge}

As discussed in Section III M, the field equations can be simplified considerably by adopting the Einstein gauge, provided $\phi \neq 0$. To obtain the corresponding forms of the field equations, one merely sets $\phi=\phi_{0}$ and makes the replacement $\left.\mathcal{D}_{a}^{\dagger} \phi\right|_{\phi=\phi_{0}}=\phi_{0}\left(\mathcal{V}_{a}+\frac{1}{3} \mathcal{T}_{a}\right)$ throughout, but we will not carry out this procedure explicitly here. As we showed earlier, however, if the resulting field equations are written in terms of $\varphi, h_{a}^{\mu}$, and the combinations $A^{\dagger a b}{ }_{\mu}$ and $V_{\mu}+\frac{1}{3} T_{\mu}$ (which will always be possible), they will be identical in form to the full field equations above expressed in terms of the scale-invariant variables $\widehat{\varphi}, \widehat{h}_{a}^{\mu}, \widehat{A}_{\mu}^{\dagger a b}$, and $\widehat{V}_{\mu}$, respectively, so that there is no need to consider the condition $\phi=\phi_{0}$ as representing a spontaneously broken symmetry.

\section{LOCALLY SCALE-INVARIANT POINCARÉ GAUGE THEORY}

In Sections II-IV, we have focussed our attention on locally scale-invariant gauge theories for which the local Poincare invariance is extended to include invariance under local changes of scale by gauging the Weyl group. In Section II, we assumed the "normal" form for the transformation of the rotational gauge field under local dilations to obtain the well-known Weyl gauge theory (WGT). This approach provides a systematic means of constructing both free gravitational and matter actions in which each term (and hence the full action in each case) is invariant under local dilations, in addition to GCT and local Lorentz rotations, by the introduction of a new (vector) gravitational gauge field related to local dilations and the construction of an associated and more general covariant derivative. Moreover, in Section III, we presented a novel alternative to WGT by considering a more general "extended" form of the transformation law of the rotational gauge field under local dilation, which includes the "normal" transformation law of WGT as a special case. The resulting "extended" Weyl gauge theory (eWGT) again relies on the introduction of a new (vector) gravitational gauge field related to these "extended" local dilations and the construction of an even more general associated covariant derivative.

As mentioned in the Introduction, however, one may construct PGTs (see Appendix B for a summary of PGT) that are already invariant under local dilations without the need to introduce an additional gravitational gauge field. This is possible assuming either the "normal" or "extended" transformation law for the rotational gauge field; we will focus on the latter here, which clearly includes the former as a special case. The construction of such locally scale-invariant PGTs is achieved by requiring the free parameters in the free gravitational action $S_{\mathrm{G}}$ and matter action $S_{\mathrm{M}}$ to obey certain relationships, so that, although each term in the actions may not be individually invariant under (extended) local dilations, $S_{\mathrm{G}}$ and $S_{\mathrm{M}}$ do each enjoy this property and hence so does the total action..$^{99}$ In Section V A, we discuss the construction of such PGTs, and in Section V B we consider locally scale-invariant reduced PGT.

\section{A. Local scale invariance in PGT}

As discussed in Section III, under a local dilation the "extended" transformations laws of the $h$ and $A$ gauge fields are given by ${h^{\prime}}_{a}{ }^{\mu}=e^{-\rho} h_{a}{ }^{\mu}$ and $A^{\prime a b}{ }_{\mu}=A^{a b}{ }_{\mu}+\theta\left(b^{a}{ }_{\mu} \mathcal{P}^{b}-b^{b}{ }_{\mu} \mathcal{P}^{a}\right)$, respectively, where $\mathcal{P}_{a}=h_{a}{ }^{\mu} \partial_{\mu} \rho$. Here $\theta=0$ recovers the "normal" transformations laws (134) and (135) assumed in WGT and $\theta=1$ corresponds to the "special" transformation law (141) for the $A$ field.

Consequently, under a local dilation, the PGT covariant derivative transforms inhomogeneously as

$$
\mathcal{D}_{c}^{\prime} \varphi^{\prime}=e^{(w-1) \rho}\left[\mathcal{D}_{c} \varphi+w \mathcal{P}_{c} \varphi+\theta \mathcal{P}^{[b} \delta_{c}^{a]} \Sigma_{a b} \varphi\right]
$$


where $w$ is the Weyl weight of the field $\varphi$ and $\Sigma_{a b}$ are the generator matrices of the Lorentz group representation to which $\varphi$ belongs. Thus, one finds that the PGT rotational gauge field strength and its contractions transform as

$$
\begin{aligned}
\mathcal{R}^{\prime a b}{ }_{c d}= & e^{-2 \rho}\left\{\mathcal{R}^{a b}{ }_{c d}+2 \theta \delta_{d}^{[a}\left(\mathcal{D}_{c}-\theta \mathcal{P}_{c}\right) \mathcal{P}^{b]}-2 \theta \delta_{c}^{[a}\left(\mathcal{D}_{d}-\theta \mathcal{P}_{d}\right) \mathcal{P}^{b]}-2 \theta \mathcal{P}^{[a} \mathcal{T}^{b]}{ }_{c d}\right. \\
& \left.-2 \theta^{2} \delta_{c}^{[a} \delta_{d}^{b]} \mathcal{P}^{e} \mathcal{P}_{e}\right\}, \\
\mathcal{R}^{\prime a}{ }_{c}= & e^{-2 \rho}\left\{\mathcal{R}^{a}{ }_{c}-2 \theta \mathcal{D}_{c} \mathcal{P}^{a}-\theta \delta_{c}^{a} \mathcal{D}_{b} \mathcal{P}^{b}+2 \theta^{2} \mathcal{P}^{a} \mathcal{P}_{c}-2 \theta^{2} \delta_{c}^{a} \mathcal{P}^{2}+\theta \mathcal{P}^{b} \mathcal{T}^{a}{ }_{c b}-\theta \mathcal{P}^{a} \mathcal{T}_{c}\right\}, \\
\mathcal{R}^{\prime}= & e^{-2 \rho}\left\{\mathcal{R}-6 \theta\left(\mathcal{D}_{a}+\frac{1}{3} \mathcal{T}_{a}\right) \mathcal{P}^{a}-6 \theta^{2} \mathcal{P}^{2}\right\},
\end{aligned}
$$

where $\mathcal{P}^{2} \equiv \mathcal{P}_{a} \mathcal{P}^{a}$, and the translational gauge field strength and its contractions transform as

$$
\begin{aligned}
\mathcal{T}^{\prime a}{ }_{b c} & =e^{-\rho}\left\{\mathcal{T}^{a}{ }_{b c}+2(1-\theta) \mathcal{P}_{[b} \delta_{c]}^{a}\right\}, \\
\mathcal{T}^{\prime}{ }_{b} & =e^{-\rho}\left\{\mathcal{T}_{b}+3(1-\theta) \mathcal{P}_{b}\right\} .
\end{aligned}
$$

Thus neither $\mathcal{R}^{a b}{ }_{c d}$ nor $\mathcal{T}^{a}{ }_{b c}$ is covariant under a general extended Weyl transformation with arbitrary $\theta$. As mentioned in Section III, however, one sees that $\mathcal{R}^{a b}{ }_{c d}$ is covariant with weight $w=-2$ under "normal" $(\theta=0)$ transformations, whereas $\mathcal{T}^{a} b c$ is covariant with weight $w=-1$ under "special" transformations $(\theta=1)$.

\section{Free gravitational action}

To construct a free gravitational action that is invariant under local dilations, one requires the gravitational Lagrangian $L_{\mathrm{G}}$ to transform covariantly with weight $w=-4$, at least up to a surface term. Assuming the extended transformation law for the $A$-field, with $\theta \neq 0$, a long but straightforward calculation shows that there is no PGT Lagrangian of the form $L_{\mathcal{R}^{2}}$ in (2) that transforms covariantly (aside from the trivial case $\alpha_{i}=0$ for all $i$ ) and so $L_{\mathrm{G}}$ must vanish. Nonetheless, by contrast, in the special case of the "normal" transformation law $(\theta=0)$ for the $A$-field, $L_{\mathrm{G}}$ may be any PGT Lagrangian of the form $L_{\mathcal{R}^{2}}$ in (2).

\section{Matter action}

To construct an appropriate matter action, we follow the rationale presented in Section II J and base our Lagrangian density on (79), but appropriately modified to use only PGT quantities. Thus, in the most general case, one might consider ${ }^{100}$

$$
\mathcal{L}_{\mathrm{M}}=h^{-1}\left[\frac{1}{2} i \bar{\psi} \gamma^{a} \stackrel{\leftrightarrow}{\mathcal{D}_{a}} \psi-\mu \phi \bar{\psi} \psi+\frac{1}{2} v\left(\mathcal{D}_{a} \phi\right)\left(\mathcal{D}^{a} \phi\right)-\lambda \phi^{4}-a \phi^{2} \mathcal{R}+\phi^{2} L_{\mathcal{T}^{2}}\right],
$$

where $\mu, v, \lambda$, and $a$ are dimensionless parameters (usually positive), and there are three further dimensionless parameters $\beta_{1}, \beta_{2}$, and $\beta_{3}$ in $L_{\mathcal{T}^{2}}$.

Let us denote (256) by $\mathcal{L}_{\mathrm{M}} \equiv \mathcal{L}_{\psi}+\mathcal{L}_{\phi}+\phi^{2} \mathcal{L}_{\mathcal{R}}+\phi^{2} \mathcal{L}_{\mathcal{T}^{2}}$. As discussed in Section III K, $\mathcal{L}_{\psi}$ is invariant under extended local dilations, but one may show that the other constituent parts of $\mathcal{L}_{\mathrm{M}}$ transform inhomogeneously as

$$
\begin{aligned}
\mathcal{L}_{\phi}^{\prime} & =\mathcal{L}_{\phi}+\frac{1}{2} v h^{-1}\left(\phi^{2} \mathcal{P}^{2}-2 \phi \mathcal{P}^{a} \mathcal{D}_{a} \phi\right), \\
\left(\phi^{2} \mathcal{L}_{\mathcal{R}}\right)^{\prime} & =\phi^{2} \mathcal{L}_{\mathcal{R}}+a \phi^{2} h^{-1} \theta\left[6\left(\mathcal{D}_{a}+\frac{1}{3} \mathcal{T}_{a}\right) \mathcal{P}^{a}+6 \theta \mathcal{P}^{2}\right], \\
\left(\phi^{2} \mathcal{L}_{\mathcal{T}^{2}}\right)^{\prime} & =\phi^{2} \mathcal{L}_{\mathcal{T}^{2}}+\left(2 \beta_{1}+\beta_{2}+3 \beta_{3}\right) \phi^{2} h^{-1}(1-\theta)\left[2 \mathcal{P}_{a} \mathcal{T}^{a}+3(1-\theta) \mathcal{P}^{2}\right] .
\end{aligned}
$$

In order to make $\mathcal{L}_{\mathrm{M}}$ invariant, one must therefore impose some conditions on the (dimensionless) parameters it contains. Indeed, a short calculation reveals a substantial simplification that is obtained by setting

$$
\frac{1}{2} v=-6 a \theta=3(\theta-1)\left(2 \beta_{1}+\beta_{2}+3 \beta_{3}\right),
$$

in which case $\mathcal{L}_{\mathrm{M}}$ transforms as

$$
\mathcal{L}_{\mathrm{M}}^{\prime}=\mathcal{L}_{\mathrm{M}}+6 a \theta h^{-1}\left(\mathcal{D}_{a}+\mathcal{T}_{a}\right)\left(\phi^{2} \mathcal{P}^{a}\right) .
$$


It is a simple matter to show that the second term on the RHS is a total derivative, and so the action based on (256) is invariant under extended local dilations with a particular value of $\theta$, provided the dimensionless parameters $v, a$, and $\beta_{i}(i=1,2,3)$ satisfy the conditions (258). It is worth noting that for a positive value of $\theta$, the parameters $v$ and $a$ must be of opposite sign, which is different to the usual case in WGT and eWGT, where they are both positive.

It is also worth noting the two special cases of a "normal" transformation $(\theta=0)$, for which one requires $v=0=2 \beta_{1}+\beta_{2}+3 \beta_{3}$, and a "special" transformation $(\theta=1)$, for which $v=0=a$. Thus, in both special cases, the kinetic term for the scalar field $\phi$ is inadmissible. Moreover, in the former case $(\theta=0)$, one obtains a generalisation of the gravitational theory proposed by Obukhov, ${ }^{89}$ who set $v=\beta_{1}=\beta_{2}=\beta_{3}=0$. Adopting our more general conditions $v=0=2 \beta_{1}+\beta_{2}+3 \beta_{3}$, one may further show that the matter Lagrangian (256) can be written as

$$
\mathcal{L}_{\mathrm{M}}=h^{-1}\left[\frac{1}{2} i \bar{\psi} \gamma^{a} \stackrel{\leftrightarrow}{\mathcal{D}}_{a} \psi-\mu \phi \bar{\psi} \psi-\lambda \phi^{4}-a \phi^{2} \mathcal{R}+\phi^{2} L_{\mathcal{T} * 2}\right],
$$

with $2 \beta_{1}+\beta_{2}+3 \beta_{3}=0$ in $L_{\mathcal{T} * 2}$, which is merely a special case of the general WGT matter Lagrangian (79).

Since the condition (258) depends on the value of the parameter $\theta$, it is clear that no single matter Lagrangian of the form (256) results in an action that is invariant under an extended local dilation for arbitrary values of $\theta$. Nonetheless, it is possible to construct such a Lagrangian using only PGT quantities by making only a minor modification to (256). Specifically, making the replacement $\mathcal{D}_{a} \phi \rightarrow\left(\mathcal{D}_{a}+\frac{1}{3} \mathcal{T}_{a}\right) \phi$ (and similarly for $\mathcal{D}^{a} \phi$ ) in the kinetic term for $\phi$, one may show that one recovers the transformation law (259) under an extended local dilation, provided only that $\frac{1}{2} v+6 a=0$ and $2 \beta_{1}+\beta_{2}+3 \beta_{3}=0$ separately. Thus, in this case, the parameters $v$ and $a$ must always be of opposite sign, rather than the usual case in WGT and eWGT, where both are positive. One may further show that these conditions allow one to write the resulting Lagrangian as

$$
\begin{aligned}
& \mathcal{L}_{\mathrm{M}}=h^{-1}\left[\frac{1}{2} i \bar{\psi} \gamma^{a} \stackrel{\leftrightarrow}{\mathcal{D}}_{a} \psi-\mu \phi \bar{\psi} \psi+\frac{1}{2} v\left(\mathcal{D}_{a}^{\dagger} \phi\right)\left(\mathcal{D}^{\dagger a} \phi\right)-\lambda \phi^{4}+\frac{1}{12} v \phi^{2} \mathcal{R}^{\dagger}+\phi^{2} L_{\mathcal{T}^{\dagger 2}}\right] \\
& -\frac{1}{2} v h^{-1}\left(\mathcal{D}_{a}+\mathcal{T}_{a}\right)\left(\phi^{2} \mathcal{V}^{a}\right),
\end{aligned}
$$

with $2 \beta_{1}+\beta_{2}=0$ in $L_{\mathcal{T} \dagger 2}$ (in which there is no term proportional to $\beta_{3}$ ). Since the final term on the RHS is a total derivative, this Lagrangian is equivalent merely to a particular special case of the eWGT matter Lagrangian (192), but one where (unusually) the kinetic term for the $\phi$-field and the $\phi^{2} \mathcal{R}^{\dagger}$ term have the same sign.

\section{Einstein gauge, scale-invariant variables, and motion of test particles}

Considering first the general case in which $\theta$ may take an arbitrary value, the matter Lagrangian must have the form (261), which is equivalent to a special case of the eWGT matter Lagrangian (192). In this case, $\mathcal{L}_{\mathrm{M}}$ must in fact be the total Lagrangian, since one requires the free gravitational Lagrangian to vanish for arbitrary $\theta$, as discussed above. Hence, our conclusions in eWGT regarding the relationship between the Einstein gauge and scale-invariant variables presented in Section III M, and the motion of test particles given in Section III N, must also hold in this case.

For the special case $\theta=0$, the matter Lagrangian (260) is an example of the general WGT matter Lagrangian (79). Similarly, the free gravitational Lagrangian in the $\theta=0$ case may be any Lagrangian of the form $L_{\mathcal{R}^{2}}$ in (2), which is just a special case of the general WGT free gravitational Lagrangian (58). It therefore follows that our conclusions in WGT regarding the relationship between the Einstein gauge and scale-invariant variables presented in Section II L, and the motion of test particles given in Section II M, also apply in this locally scale-invariant PGT.

\section{B. Local scale-invariance in reduced PGT}

We now consider the construction of locally scale-invariant reduced PGTs. As discussed in Appendix B (see also Section II E), in the "reduced" PGT covariant derivative ${ }^{0} \mathcal{D}_{a}$, to which the full PGT covariant derivative reduces for vanishing PGT torsion, the rotational gauge field 
$A^{a b}{ }_{\mu}$ is replaced by ${ }^{0} A^{a b}{ }_{\mu}$, which depends only on the $h$-field and its first derivatives. This covariant derivative may be used to construct the "reduced" rotational field strength (or "curvature") tensor ${ }^{0} \mathcal{R}^{a b}{ }_{c d}$, which depends only on the $h$-field and its first and second derivatives. Moreover, the corresponding reduced translational field strength (or "torsion") tensor vanishes, since ${ }^{0} \mathcal{T}^{a}{ }_{b c} \equiv h_{b}{ }^{\mu} h_{c}{ }^{v}\left({ }^{0} D_{\mu} b^{a}{ }_{v}-{ }^{0} D_{\nu} b^{a}{ }_{\mu}\right)=0$. Thus, reduced PGTs can be expressed entirely in terms of the $h$-field and its derivatives and correspond to imposing the condition of vanishing PGT torsion directly at the level of the action.

One may show that, under an extended local dilation, ${ }^{0} A^{\prime a b}{ }_{\mu}={ }^{0} A^{a b}{ }_{\mu}+b^{a}{ }_{\mu} \mathcal{P}^{b}-b^{b}{ }_{\mu} \mathcal{P}^{a}$, which is independent of $\theta$, as is expected since ${ }^{0} A^{a b}{ }_{\mu}$ depends on only the $h$-field (and its first derivatives), for which $h^{\prime}{ }^{\mu}{ }^{\mu}=e^{-\rho} h_{a}{ }^{\mu}$. Thus, the "normal" and "extended" transformations laws for ${ }^{0} A^{a b}{ }_{\mu}$ coincide. The corresponding "reduced" PGT covariant derivative ${ }^{0} \mathcal{D}_{a}$ transforms inhomogeneously under a local dilation as

$$
{ }^{0} \mathcal{D}_{c}^{\prime} \varphi^{\prime}=e^{(w-1) \rho}\left[{ }^{0} \mathcal{D}_{c} \varphi+w \mathcal{P}_{c} \varphi+\mathcal{P}^{[b} \delta_{c}^{a]} \Sigma_{a b} \varphi\right],
$$

where $w$ is the Weyl weight of the field $\varphi$ and $\Sigma_{a b}$ are the generator matrices of the Lorentz group representation to which $\varphi$ belongs. Similarly, one finds that the "reduced" rotational field strength transforms as

$$
\begin{aligned}
{ }^{0} \mathcal{R}^{\prime a b}{ }_{c d} & =e^{-2 \rho}\left\{{ }^{0} \mathcal{R}^{a b}{ }_{c d}+2 \delta_{d}^{[a}\left({ }^{0} \mathcal{D}_{c}-\mathcal{P}_{c}\right) \mathcal{P}^{b]}-2 \delta_{c}^{[a}\left({ }^{0} \mathcal{D}_{d}-\mathcal{P}_{d}\right) \mathcal{P}^{b]}-2 \delta_{c}^{[a} \delta_{d}^{b]} \mathcal{P}^{e} \mathcal{P}_{e}\right\}, \\
{ }^{0} \mathcal{R}^{\prime a}{ }_{c} & =e^{-2 \rho}\left\{{ }^{0} \mathcal{R}^{a}{ }_{c}-2{ }^{0} \mathcal{D}_{c} \mathcal{P}^{a}-\delta_{c}^{a}{ }^{0} \mathcal{D}_{b} \mathcal{P}^{b}+2 \mathcal{P}^{a} \mathcal{P}_{c}-2 \delta_{c}^{a} \mathcal{P}^{2}\right\}, \\
{ }^{0} \mathcal{R}^{\prime} & =e^{-2 \rho}\left\{{ }^{0} \mathcal{R}-6{ }^{0} \mathcal{D}_{a} \mathcal{P}^{a}-6 \mathcal{P}^{2}\right\},
\end{aligned}
$$

and ${ }^{0} \mathcal{T}^{a}{ }_{b c}$ remains self-consistently zero. It is worth noting that, under a local dilation, the "reduced" quantities ${ }^{0} A^{a b}{ }_{\mu},{ }^{0} \mathcal{D}_{c} \varphi$, and ${ }^{0} \mathcal{R}^{a b}{ }_{c d}$ all transform independently of $\theta$, and in the same way as their "full" PGT counterparts $A^{a b}{ }_{\mu}, \mathcal{D}_{c} \varphi$, and $\mathcal{R}^{a b}{ }_{c d}$ do assuming the "special" transformation law (141) for the $A$-field $(\theta=1)$, but with the replacements $\mathcal{D}_{c} \rightarrow{ }^{0} \mathcal{D}_{c}$ and $\mathcal{T}^{a} b c$ $\rightarrow{ }^{0} \mathcal{T}^{a}{ }_{b c} \equiv 0$.

\section{Free gravitational action}

Following the discussion in Appendix B, if $L_{G}$ contains only terms that are at most quadratic in ${ }^{0} \mathcal{R}^{a b}{ }_{c d}$ and its contractions, then it has the general form (B3). For $L_{G}$ to transform covariantly under local dilations, however, it cannot contain the dimensionful constants $\kappa$ and $\Lambda$, and so the most general form one need to consider is that given in (B4), namely,

$$
L_{\mathrm{G}}=\alpha_{1}{ }^{0} \mathcal{R}^{2}+\alpha_{2}{ }^{0} \mathcal{R}_{a b}{ }^{0} \mathcal{R}^{a b}+\alpha_{3}{ }^{0} \mathcal{R}_{a b c d}{ }^{0} \mathcal{R}^{a b c d},
$$

where the $\alpha_{i}$ are dimensionless free parameters. As mentioned in Appendix B, one can use the Gauss-Bonnet identity to set to zero any one of the parameters $\alpha_{i}$, with no loss of generality (at least classically). For our current purposes, however, it is more convenient to retain the form (264).

Using the expressions (263a)-(263c), a short calculation reveals that $L_{G}$ transforms covariantly with weight $w=-4$ under an extended local dilation, provided $2 \alpha_{3}+\alpha_{2}=0$ and $3 \alpha_{1}-\alpha_{3}=0$. Letting $\alpha=\alpha_{3}$ for convenience, one thus obtains the one-parameter free gravitational Lagrangian

$$
L_{\mathrm{G}}=\alpha\left({ }^{0} \mathcal{R}_{a b c d}{ }^{0} \mathcal{R}^{a b c d}-2^{0} \mathcal{R}_{a b}{ }^{0} \mathcal{R}^{a b}+\frac{1}{3}^{0} \mathcal{R}^{2}\right),
$$

for which the corresponding action is thus unique up to surface terms and an overall scaling. If one wishes, one may now use the fact that the Gauss-Bonnet term (59) contributes a total derivative to the action (in $D \leq 4$ dimensions) to remove terms in $L_{G}$. The calculation of the field equations is simplest if one removes the term proportional to ${ }^{0} \mathcal{R}_{a b c d}{ }^{0} \mathcal{R}^{a b c d}$ to obtain

$$
L_{\mathrm{G}}=2 \alpha\left({ }^{0} \mathcal{R}_{a b}{ }^{0} \mathcal{R}^{a b}-\frac{1}{3}{ }^{0} \mathcal{R}^{2}\right),
$$

which changes only by a surface term under local changes of scale, and hence, the equations of motions are covariant under such a transformation. 
When interpreted geometrically, the theory defined by (265) or (266) coincides with so-called conformal gravity theory. ${ }^{101}$ Indeed, as one might suspect, one can easily show that (265) is proportional to the Weyl-squared Lagrangian $L_{\mathrm{W}}={ }^{0} C_{a b c d} C^{a b c d}$, where ${ }^{0} C_{a b c d}$ is the gauge theory equivalent of the Weyl tensor, given by

${ }^{0} \mathcal{C}_{a b c d}={ }^{0} \mathcal{R}_{a b c d}-\frac{1}{2}\left(\eta_{a c}{ }^{0} \mathcal{R}_{b d}-\eta_{a d}{ }^{0} \mathcal{R}_{b c}-\eta_{b c}{ }^{0} \mathcal{R}_{a d}+\eta_{b d}{ }^{0} \mathcal{R}_{a c}\right)+\frac{1}{6}\left(\eta_{a c} \eta_{b d}-\eta_{a d} \eta_{b c}\right)^{0} \mathcal{R}$.

This tensor represents the traceless part of ${ }^{0} \mathcal{R}_{a b c d}$; it has the same symmetries, but satisfies the extra condition of being trace-free (contraction on any pair of indices yields zero). It transforms covariantly as ${ }^{0} C_{a b c d}^{\prime}=e^{-2 \rho 0} C_{a b c d}$ under a local change of scale, so that $L_{W}$ also transforms covariantly with a Weyl weight $w\left(L_{W}\right)=-4$, and hence the corresponding action is invariant under a local change of scale, as found above.

It should be noted, however, that the Lagrangian (265) or (266) is quadratic in second-order derivatives of the $h$-field, and so the corresponding field equations will typically be linear in fourth-order derivatives of $h$. This suggests that conformal gravity may suffer from Ostrogradsky's instability, although this needs to be checked in detail. Ostrogradsky's instability is often reinterpreted as non-unitary behaviour in the associated quantum theory, but this instability occurs in the classical Hamiltonian and survives canonical quantisation; ${ }^{19}$ it thus seems unlikely that it can be circumvented by any attempt to redefine the Fock space of the quantum theory. ${ }^{102}$ Such issues may be problematic for conformal gravity as a fundamental description of the gravitational field, although further investigation is clearly necessary.

\section{Matter action}

To construct an appropriate matter action, we again follow the rationale presented in Section II J, but appropriately modified to use "reduced" PGT quantities relevant to the special case of vanishing PGT torsion. Thus, in the most general case, one might consider the Lagrangian (256), but with the replacements $\mathcal{D}_{a} \rightarrow{ }^{0} \mathcal{D}_{a}, \mathcal{R} \rightarrow{ }^{0} \mathcal{R}$, and without the term proportional to $L_{\mathcal{T}^{2}}$, namely, ${ }^{103}$

$$
\mathcal{L}_{\mathrm{M}}=h^{-1}\left[\frac{1}{2} i \bar{\psi} \gamma^{a}{\stackrel{\leftrightarrow}{\mathcal{D}_{a}} \psi}_{a}-\mu \phi \bar{\psi} \psi+\frac{1}{2} v\left({ }^{0} \mathcal{D}_{a} \phi\right)\left({ }^{0} \mathcal{D}^{a} \phi\right)-\lambda \phi^{4}-a \phi^{2}{ }^{0} \mathcal{R}\right] .
$$

Using (262) and (263c), one may show that, under a local change of scale, $\mathcal{L}_{\mathrm{M}}$ transforms as

$$
\mathcal{L}_{\mathrm{M}}^{\prime}=\mathcal{L}_{\mathrm{M}}-h^{-1}\left[\frac{1}{2} \nu \mathcal{P}^{a} 0 \mathcal{D}_{a} \phi^{2}-6 a \phi^{20} \mathcal{D}_{a} \mathcal{P}^{a}+\left(\frac{1}{2} \nu+6 a\right) \phi^{2} \mathcal{P}_{a} \mathcal{P}^{a}\right]
$$

By setting $\frac{1}{2} v+6 a=0$, however, one obtains

$$
\mathcal{L}_{\mathrm{M}}^{\prime}=\mathcal{L}_{\mathrm{M}}-h^{-1}\left[\frac{1}{2} \nu^{0} \mathcal{D}_{a}\left(\phi^{2} \mathcal{P}^{a}\right)\right]
$$

where we recognise the second term on the RHS as a total derivative. Thus, in this special case, the corresponding field equations are covariant under local dilations. The resulting matter Lagrangian, obtained by setting $\frac{1}{2} v+6 a=0$ in (268), coincides with that typically assumed in conformal gravity. ${ }^{101}$ It is worth noting that, as found in Sec. V A 2, the dimensionless parameters $v$ and $a$ must have opposite signs, which is different to the usual case in WGT and eWGT, where both parameters are positive. Thus, the resulting matter Lagrangian is again unusual in having the same sign for the kinetic term for $\phi$ and the $\phi^{20} \mathcal{R}$ term.

\section{Einstein gauge, scale-invariant variables, and motion of particles}

One may show that the definition (267) of the Weyl tensor ${ }^{0} C_{a b c d}$ also holds if one makes either of the replacements ${ }^{0} \mathcal{R}_{a b c d} \rightarrow{ }^{0} \mathcal{R}_{a b c d}^{*}$ or ${ }^{0} \mathcal{R}_{a b c d} \rightarrow{ }^{0} \mathcal{R}_{a b c d}^{\dagger}$ (and similarly for the associated contractions) on the RHS, thereby replacing the "reduced" curvature tensor in PGT with its counterpart either in WGT or eWGT. Thus, the free gravitational Lagrangian (265) retains its form if one makes either set of replacements and is hence a special case of the free gravitational Lagrangians in both reduced WGT and reduced eWGT, namely, (111) and (203), respectively.

Similarly, the matter Lagrangian (268) with $\frac{1}{2} v+6 a=0$ may be shown to equal (261) with $\mathcal{D}_{a} \rightarrow{ }^{0} \mathcal{D}_{a}$ and $\mathcal{T}_{a} \equiv 0$ and either set of replacements $\mathcal{D}_{a}^{\dagger} \rightarrow{ }^{0} \mathcal{D}_{a}^{*}, \mathcal{R}^{\dagger} \rightarrow{ }^{0} \mathcal{R}^{*} \mathcal{T}_{a b c}^{\dagger} \rightarrow{ }^{0} \mathcal{T}_{a b c}^{*} \equiv 0$ or 
$\mathcal{D}_{a}^{\dagger} \rightarrow{ }^{0} \mathcal{D}_{a}^{\dagger}, \mathcal{R}^{\dagger} \rightarrow{ }^{0} \mathcal{R}^{\dagger} \mathcal{T}_{a b c}^{\dagger} \rightarrow{ }^{0} \mathcal{T}_{a b c}^{\dagger} \equiv 0$. Thus, (268) with $\frac{1}{2} v+6 a=0$ is equivalent to a special case of the general matter Lagrangian in both reduced WGT and reduced eWGT, namely, (79) and (192), respectively, with vanishing WGT/eWGT torsion.

As a result, our conclusions regarding the equivalence of the Einstein gauge and scale-invariant variables, and the motion of test particles, presented in Sections II L and II M for WGT and Sections III M and III N for eWGT, also hold in this case.

\section{DISCUSSION AND CONCLUSIONS}

We have presented a general approach to constructing gauge theories of gravity in a manner that differs from that usually presented in the literature. In particular, in keeping with the field theories that describe the other fundamental interactions, we maintain throughout the notion of gauge fields in Minkowski spacetime, rather than adopting the more usual geometric interpretation, which has some non-trivial consequences. We also work exclusively in terms of finite local transformations, rather than their usual infinitesimal forms; this allows, in our view, for a more transparent interpretation of the resulting theories.

We focus our attention in particular on constructing locally scale-invariant gauge theories of gravity. This is usually achieved by extending local Poincaré invariance to include invariance under local changes of scale by gauging Weyl transformations. The resulting Weyl gauge theory (WGT) is well known and generalises Poincaré gauge theory (PGT) by providing a systematic gauge-theoretic means of constructing both matter and gravitational actions that are invariant under local scale transformations, in addition to GCT and local Lorentz rotations, by the introduction of a new (vector) gravitational gauge field related to local dilations and the construction of an associated and more general covariant derivative. The WGT action also typically depends on a scalar compensator field $\phi$, which opens up the possibility of including terms in which $\phi$ is non-minimally coupled to the gauge field strength tensors, in addition (usually) to kinetic and self-interaction terms for $\phi$. Although WGT has been widely studied, we make some observations that differ from typical accounts of the subject, in particular regarding the interpretation of the Einstein gauge and also the equations of motion of matter fields and test particles. In the Einstein gauge, one simplifies the equations of motion by using local scale-invariance to set the compensator scalar field to a constant, $\phi(x)=\phi_{0}$. This is usually considered as representing the choice of some definite scale in the theory and is often given the physical interpretation of corresponding to some spontaneous breaking of the scale symmetry. We show, however, that the equations of motion in the Einstein gauge are identical in form to those obtained when working in terms of scale-invariant variables, where the latter involves no breaking of the scale symmetry. This suggests that one should introduce further scalar fields, in addition to the compensator field $\phi$, to enable a true breaking of the scale symmetry. Regarding the trajectories of test particles in WGT (and PGT as a special case), we construct an action for a classical spin- $\frac{1}{2}$ point particle, which we use as our model for "ordinary matter," and show that such particles satisfy an equation of motion that corresponds to geodesic paths, rather than autoparallels; we also show that the same is true for photons.

Our main objective, however, is to present a novel alternative to WGT by considering a more general "extended" transformation law for the rotational gauge field under local dilations, which includes its "normal" transformation law in WGT as a special case. This is motivated by the observation that the PGT (and WGT) matter actions both for the Dirac field and the electromagnetic field are already invariant under local dilations if one assumes this "extended" transformation law in the same way as they are for the "normal" transformation law assumed in WGT. Moreover, under a global scale transformation, the two transformation laws coincide, and so both may be considered as equally valid gauging of global Weyl scale invariance. The key difference between the two sets of transformations is that, whereas under the "normal" Weyl transformations the PGT gauge fields $h_{a}{ }^{\mu}$ and $A^{a b}{ }_{\mu}$ transform covariantly with weights of $w,-1$ and 0 , respectively, under the "extended" transformations, the rotational gauge field $A^{a b}{ }_{\mu}$ transforms inhomogeneously. This has the consequence that the transformation properties of the PGT rotational gauge field strength (or "curvature") $\mathcal{R}_{a b c d}$ and translational gauge field strength (or "torsion") $\mathcal{T}_{a b c}$ are treated in a 
more balanced manner. The resulting "extended" Weyl gauge theory (eWGT) again relies on the introduction of a new (vector) gravitational gauge field $V_{\mu}$ related to the "extended" local dilations and the construction of an associated covariant derivative $\mathcal{D}_{a}^{\dagger}$.

Our extended WGT has a number of interesting features, which include the following.

- The translational, rotational, and dilational field strength tensors $\mathcal{R}_{a b c d}^{\dagger}(h, A, \partial A, V, \partial V), \mathcal{T}_{a b c}^{\dagger}$ $(h, \partial h, A)$, and $\mathcal{H}_{a b}^{\dagger}\left(h, \partial h, \partial^{2} h, A, \partial A, \partial V\right)$, respectively, depend on the gauge fields $h_{a}{ }^{\mu}, A^{a b}{ }_{\mu}$, and $V_{\mu}$ and their derivatives in a profoundly different way to WGT, as indicated, leading to a very different dependence of the free gravitational and matter actions on the gauge fields.

- An action that is at most quadratic in the field strength tensors $\mathcal{R}_{a b c d}^{\dagger}$ and $\mathcal{T}_{a b c}^{\dagger}$, respectively, yields equations of motion that are, in general, linear in the second derivatives of the gauge fields, and hence, the corresponding Hamiltonian does not suffer from Ostrogradsky's instability. If one includes a term quadratic in $\mathcal{H}_{a b}^{\dagger}$, however, it contributes terms to the resulting field equations that are linear in fourth-order derivatives of the $h_{a}{ }^{\mu}$ field. Nonetheless, as we discussed in Section IV B, there are grounds for omitting the term quadratic in $\mathcal{H}_{a b}^{\dagger}$ from the action and, moreover, even if this term is included, the theory may still not suffer from Ostrogradsky's instability.

- The trace $\mathcal{T}_{a}^{\dagger}$ of the eWGT translational gauge field strength vanishes identically, which has a number of consequences. These include the automatic equivalence of the minimal coupling procedure when applied at the level of the action or directly in the field equations; a simplified torsion-squared part of the free gravitational action; and simplified Bianchi identities. In particular, the covariant divergence of the translation gauge field strength depends only on the rotational and dilational gauge field strengths.

- The energy-momentum tensors $t_{a b}$ and $\tau_{a b}$ derived from the free gravitational and matter actions, respectively, are not separately covariant under extended local dilations, although their non-covariant parts cancel to yield a covariant $h$-field equation, as required. Nonetheless, one can construct covariant versions $t_{a b}^{\dagger}$ and $\tau_{a b}^{\dagger}$ of free-gravitational and matter energy momentum tensors; this is most naturally achieved by adopting an alternative variational principle in which one makes a change of field variables such that $A^{a b}{ }_{\mu}$ is replaced by $A^{\dagger a b}{ }_{\mu} \equiv A^{a b}{ }_{\mu}+\left(\mathcal{V}^{a} b^{b}{ }_{\mu}-\mathcal{V}^{b} b^{a}{ }_{\mu}\right)$.

- In terms of this alternative set of field variables, the total Lagrangian density does not depend explicitly on the dilation gauge field $V_{\mu}$ or its derivatives. This leads to the identification of an alternative dilation current $\zeta_{a}^{\dagger}$, which vanishes identically. As a consequence, the contribution of the free gravitational and matter sectors, respectively, to the $V$-field equation is merely twice the relevant contraction of their contributions to the $A$-field equation.

- The conservation equations have a very different form to those in PGT or WGT. In particular, invariance of the free gravitational action under extended local dilations leads to a differential conservation law similar to that obtained in WGT, but also an additional algebraic condition that the trace $t^{\dagger a}{ }_{a}$ of the free-gravitational energy momentum tensor (i.e., the trace of the gravitational sector's contribution to the $h$-field equation) should vanish. Similarly, in the matter sector, one obtains an additional algebraic condition on the trace $\tau^{\dagger a}{ }_{a}$ of the total matter energy-momentum tensor in terms of the equations of motion of the matter field $\varphi$ and (compensator) scalar field $\phi$.

- The combination of the previous two results leads to eWGT having only three independent field equations, namely, the $h$-equation, the $A$-equation, and the $\varphi$-equation. In this respect, eWGT is more similar to PGT than standard WGT.

- The conclusions given above regarding the interpretation of the Einstein gauge and the equations of motion of test particles in WGT are also found to hold in eWGT.

- In the special case of identically vanishing eWGT translational gauge field strength, $\mathcal{T}_{a b c}^{\dagger} \equiv 0$, the eWGT covariant derivative $\mathcal{D}_{a}^{\dagger}$ simplifies to a "reduced" form ${ }^{0} \mathcal{D}_{a}^{\dagger}$ that has a very different structure to that in WGT, since it still depends on the rotational gauge field $A^{a b}{ }_{\mu}$.

- One may use the "reduced" covariant derivative ${ }^{0} \mathcal{D}_{a}^{\dagger}$ to construct "reduced" eWGT, which corresponds to imposing the condition of vanishing eWGT torsion directly at the level of 
the action. Unlike reduced PGT and WGT, the resulting theory, and in particular the reduced rotational field strength tensor ${ }^{0} \mathcal{R}_{a b c d}^{\dagger}\left(h, \partial h, \partial^{2} h, A, \partial A, V, \partial V\right)$, depends on all the gauge fields, most notably the rotational $A$-gauge field. As in reduced PGT and WGT, however, the rotational field strength tensor contains second-order derivatives of the $h$-field, so that terms in the action that are quadratic in ${ }^{0} \mathcal{R}_{a b c d}^{\dagger}$ will contribute terms to the resulting equations of motion that are linear in fourth-order derivatives of $h$, and such theories typically suffer from Ostrogradsky's instability. It is important, however, to distinguish reduced eWGT from simply imposing the condition $\mathcal{T}_{a b c}^{\dagger}=0$ in the field equations of eWGT, which yields different equations of motion in general, and a theory that does not suffer from Ostrogradsky's instability, if the term proportional to $\mathcal{H}_{a b}^{\dagger} \mathcal{H}^{\dagger a b}$ is excluded from the free gravitational Lagrangian.

- The geometric interpretation of eWGT is in terms of a new spacetime geometry that represents an extension of Weyl-Cartan $Y_{4}$ spacetime.

- The extended transformation law introduced for the rotational gauge field $A^{a b}{ }_{\mu}$, and the associated introduction of its modified counterpart $A^{\dagger a b}{ }_{\mu}$, implements Weyl scaling in a novel way that may be related to gauging of the full conformal group, although this remains a topic for future research.

We have also explicitly presented a new scale-invariant gauge theory of gravity, defined by the most general parity-invariant eWGT Lagrangian that is at most quadratic in the eWGT field strengths and can accommodate "ordinary" matter. We derive the field equations for this theory and comment briefly on their structure. The phenomenology of eWGT and its application to astrophysics and cosmology will be described in forthcoming papers.

Finally, we consider the construction of PGT actions that are invariant under local dilations, focussing on the extended transformation law for the rotational gauge field (which includes as special case the normal transformation law usually assumed in WGT). After considering the general case, we discuss the construction of locally scale-invariant reduced PGT and identify the unique resulting theory as equivalent to conformal gravity, when interpreted geometrically, but note that this theory may suffer from Ostrogradsky's instability. We show that, in general, locally scale-invariant (reduced) PGTs are merely special cases of (reduced) WGT or eWGT, depending on the transformation law assumed for the rotational gauge field.

\section{ACKNOWLEDGMENTS}

The authors thank Steve Gull, Chris Doran, and Paul Alexander for numerous interesting discussions of gravity over many years and the anonymous referee for several insightful suggestions.

\section{APPENDIX A: SEMI-CLASSICAL MODEL FOR A SPINNING POINT PARTICLE}

The dynamics of a fermion is described by the Dirac equation, together with the quantummechanical rules for constructing observables. For many applications, however, such as determining the motion of a massive matter particle, it is useful to work with semi-classical and classical approximations to the full quantum theory, which we summarise here.

\section{Re-expression of the Dirac Lagrangian}

The standard special-relativistic Lagrangian for a classical Dirac field $\psi$ of mass $m$ is given by

$$
L_{\mathrm{D}}=\frac{1}{2} i\left[\bar{\psi} \gamma^{\mu} \partial_{\mu} \psi-\left(\partial_{\mu} \bar{\psi}\right) \gamma^{\mu} \psi\right]-m \bar{\psi} \psi \equiv \mathfrak{R}(i \bar{\psi} \not \partial \psi)-m \bar{\psi} \psi,
$$

where the kinetic energy term is written so that it is manifestly real (and the sign ensures that the terms contribute positive energy on quantisation) and $\not \partial \equiv \gamma^{\mu} \partial_{\mu}$. Our aim here is to rewrite the kinetic term in (A1) in an alternative form by obtaining an identity that will allow us to replace $\bar{\psi}$. 
We begin by considering the quantity $\bar{\psi} \not$, where $J^{\mu}=\bar{\psi} \gamma^{\mu} \psi$ is the Dirac current, which we may write as

$$
\bar{\psi} \phi=J^{\mu} \bar{\psi} \gamma_{\mu}=\bar{\psi} \gamma^{\mu} \psi \bar{\psi} \gamma_{\mu} .
$$

The spinor outer product $\psi \bar{\psi}$ may be rewritten using the Fierz rearrangement formula as

$$
\psi \bar{\psi}=\frac{1}{4}\left(\bar{\psi} \psi+\bar{\psi} \gamma^{v} \psi \gamma_{\nu}-\bar{\psi} \gamma^{v} \gamma^{5} \psi \gamma_{v} \gamma^{5}+\frac{1}{2} \bar{\psi} \sigma^{v \rho} \psi \sigma_{v \rho}+\bar{\psi} \gamma^{5} \psi \gamma^{5}\right)
$$

where $\gamma^{5}=\gamma_{5}=i \gamma^{0} \gamma^{1} \gamma^{2} \gamma^{3}$ and $\sigma^{v \rho}=\frac{i}{2}\left[\gamma^{\nu}, \gamma^{\rho}\right]$ (and we have assumed the spinor components to be normal $c$-numbers, rather than Grassmann variables). Inserting (A3) into (A2), one finds after a short calculation that

$$
\bar{\psi} \not \supset=\bar{\psi}\left(\bar{\psi} \psi+\bar{\psi} i \gamma^{5} \psi i \gamma^{5}\right) .
$$

It is convenient to define the real scalar $\rho \equiv \bar{\psi} \psi$ and real pseudoscalar $\beta \equiv \bar{\psi} i \gamma^{5} \psi$, so that (A4) reads $\bar{\psi} f=\bar{\psi}\left(\rho+\beta i \gamma^{5}\right)$. Postmultiplying this expression by $\left(\rho-\beta i \gamma^{5}\right)$ and rearranging, one finds that $\bar{\psi}$ can be written as

$$
\bar{\psi}=\frac{\bar{\psi}\left(\rho+\beta i \gamma^{5}\right) \not}{\rho^{2}+\beta^{2}} .
$$

Inserting this form into the kinetic part of the Dirac Lagrangian (A1) and writing the result explicitly in terms of $\psi$ and $\bar{\psi}$, one finally obtains the re-expressed form

$$
L_{\mathrm{D}}=\frac{\mathfrak{R}\left[i \bar{\psi}\left(\bar{\psi} \psi+\bar{\psi} i \gamma^{5} \psi i \gamma^{5}\right) \not \not \partial \psi\right]}{(\bar{\psi} \psi)^{2}+\left(\bar{\psi} i \gamma^{5} \psi\right)^{2}}-m \bar{\psi} \psi
$$

In particular, we note that the quantity $\not \partial \psi$ appearing in (A6) may be written as

$$
\not \partial \not \psi=J^{\mu} \partial_{\mu} \psi-i \sigma^{\mu \nu} J_{\mu} \partial_{\nu} \psi \text {. }
$$

The quantity $J^{\mu} \partial_{\mu} \psi$ is clearly interpreted as a derivative along a streamline of the Dirac fluid. Similarly, the remaining term on the RHS of (A7) is a derivative along the direction $n^{\nu} \equiv-i \sigma^{\mu \nu} J_{\mu}$, which is orthogonal to $J^{\mu}$, since $J_{v} n^{v}=-i \sigma^{\mu \nu} J_{\mu} J_{v}=0$. Thus, $-i \sigma^{\mu \nu} J_{\mu} \partial_{\nu} \psi$ is interpreted as a derivative perpendicular to a streamline of the Dirac fluid.

\section{Action for a spin- $\frac{1}{2}$ point particle}

Our reason for rewriting the Dirac Lagrangian in the alternative form (A6) is that it lends itself to the construction of an action for a classical spin- $\frac{1}{2}$ point particle, which we use as our model for "ordinary matter." The essential idea ${ }^{9}$ is to specialise to motion along a single streamline defined by the Dirac current $J^{\mu}$. Thus the particle is described by a worldline $x^{\mu}(\lambda)$, together with a "rotor" $R(\lambda)$, which is a Dirac spinor defined along the worldline that satisfies the normalisation conditions $\bar{R} R=1$ and $\bar{R} i \gamma^{5} R=0$, and contains information about the velocity and spin of the particle.

Thus, to obtain the Lagrangian for a point particle, one replaces $\psi$ by $R$ in (A6), restricts derivatives to lie purely along the particle worldline, and identifies the velocity $\dot{x}^{\mu} \equiv d x^{\mu} / d \lambda$ with $\bar{R} \gamma^{\mu} R$. The last identification is enforced by including in the action integral a Lagrange multiplier $p_{\mu}(\lambda)$, which is identified with the momentum of the particle; note that, in general, $p_{\mu}$ and $\dot{x}^{\mu}$ are not collinear. Finally, an einbein $e(\lambda)$ is introduced (written as $m e(\lambda)$ for later convenience) to ensure reparameterisation invariance along the particle worldline. The resulting action may be written as

$$
S=\int d \lambda\left[\Re(i \bar{R} \dot{R})-p_{\mu}\left(\dot{x}^{\mu}-m e \bar{R} \gamma^{\mu} R\right)-m^{2} e\right] .
$$

An equivalent expression has been derived previously, ${ }^{9}$ but there the action is instead expressed in terms of geometric algebra. Variation of the action with respect to the dynamical variables $x^{\mu}(\lambda)$, $R(\lambda), p^{\mu}(\lambda)$, and $e(\lambda)$ leads to the semi-classical equations of motion for a spin- $\frac{1}{2}$ point particle. ${ }^{104}$

One proceeds to the full classical approximation by replacing $\bar{R} \gamma^{\mu} R$ by $p^{\mu} / m$ and then neglecting the particle spin by dropping all terms that contain $R$. Redefining the einbein $e(\lambda) \rightarrow \frac{1}{2} e(\lambda)$ for 
later convenience, this process leads to the action

$$
S=-\int d \lambda\left[p_{\mu} \dot{x}^{\mu}-\frac{1}{2} e\left(p_{\mu} p^{\mu}-m^{2}\right)\right]
$$

and variation with respect to the remaining dynamical variables $x^{\mu}(\lambda), p^{\mu}(\lambda)$, and $e(\lambda)$ leads to the classical equations of motion for the point particle.

\section{APPENDIX B: POINCARÉ GAUGE THEORY}

The discussion of WGT in Section II also serves, with appropriate notational modifications, as an account of PGT, which may be considered as a special case. In particular, by setting $\rho=0$ in the global Weyl transformation (6), one recovers a global Poincaré transformation. Thus, all the subsequent results in Section II A relating to global Weyl transformations are also valid for global Poincaré transformations provided one sets $\rho=0$.

In an analogous manner, local Weyl transformations reduce to local Poincaré transformations if $\rho(x)=0$. In this case, it is no longer necessary to introduce the dilation gauge field $B_{\mu}$ to construct the PGT $\Lambda$-covariant derivative $D_{\mu} \varphi$. Thus, all the results given in Sections II B and II C also hold in PGT if one sets $\rho(x)=0=B_{\mu}$ and removes all asterisks from derivative operators. In particular, it is worth noting that the rotational gauge field $A^{a b}{ }_{\mu}$ transforms identically in PGT and WGT, according to (23).

Similar considerations apply to field strength tensors. Indeed, all the results presented in Section II D are also valid in PGT if one sets $B_{\mu}=0$ and removes all asterisks. In particular, it is worth noting that the rotational field strength tensor $R^{a b}{ }_{\mu \nu}$ in (39) has the same form in both PGT and WGT (and so no distinction is made between them), whereas the dilation field strength $H_{\mu \nu}$ in (40) vanishes identically in PGT. Also, the translation field strengths in PGT and WGT are related by (44).

Equally, the results presented in Section II E regarding the construction of a "reduced" covariant derivative also hold in PGT if one again sets $B_{\mu}=0$ and removes all asterisks. Moreover, the decomposition (46) or (47) holds with all asterisks removed from the RHS. Consequently, in the special case where the equations of motion result in $\mathcal{T}_{a b c}$ being independent of $\mathcal{A}_{a b c}$, one obtains an explicit expression for the $A$-field in terms of just the $h$-gauge field. Turning to the Bianchi identities, the same prescription of setting $B_{\mu}=0$ (so that $H_{\mu v}=0$ ) and removing all asterisks from the results given in Section II F leads to the corresponding identities in PGT.

In constructing a free gravitational Lagrangian for PGT, one has considerably more freedom than in the WGT case discussed in Section II G, since one requires only that $L_{\mathrm{G}}$ is a scalar function of the two PGT field strengths $\mathcal{R}_{a b c d}$ and $\mathcal{T}_{a b c}$ and there is no restriction on its Weyl weight $w$. Thus, in principle, $L_{\mathrm{G}}$ may contain terms of any order in the field strengths. As discussed in the Introduction, however, if one demands adequacy of kinematics and dynamics, and consistency with the standard Einstein general relativity in the macroscopic limit, one is led naturally to a Lagrangian $L_{\mathrm{G}}$ that is, at most, quadratic in the field strength tensors, such that

$$
L_{\mathrm{G}}=-\kappa^{-1}(\Lambda+a \mathcal{R})+L_{\mathcal{R}^{2}}+\kappa^{-1} L_{\mathcal{T}^{2}},
$$

where $\mathcal{R} \equiv \mathcal{R}^{a b}{ }_{a b}, \Lambda$ is a cosmological constant, $a$ is a dimensionless free parameter (usually positive), and $L_{\mathcal{R}^{2}}$ and $L_{\mathcal{T}^{2}}$ are given by (2) and (3), respectively. As in WGT, the field strength $\mathcal{R}_{a b c d}$ satisfies a form of the Gauss-Bonnet identity, such that the combination in (59) contributes a total derivative to the action (in $D \leq 4$ dimensions). Hence one may set any one of $\alpha_{1}, \alpha_{3}$, or $\alpha_{6}$ in (2) to zero, without loss of generality (at least classically). The precise version of PGT under consideration depends on the choice of the parameters $a, \Lambda,\left\{\alpha_{i}\right\}$, and $\left\{\beta_{i}\right\}$ in $L_{\mathrm{G}}$. As an illustration, in Appendix $\mathrm{C}$ we give a brief account of the form of the theory first considered by Kibble, in which $L_{\mathrm{G}} \propto \mathcal{R}$.

The total action $S_{\mathrm{T}}$ is simply the sum of the free gravitational and matter actions. In the free gravitational sector, adopting the usual form of the Lagrangian (B1) induces dependencies on both gauge fields $h$ and $A$ (suppressing indices for brevity), and their first derivatives. In particular, the dependence on $\partial h$ results from the presence of $L_{\mathcal{T}^{2}}$ in (B1), which is absent in WGT. In the matter 
sector, covariant derivatives of the matter field $\varphi$ induce a dependence on $\varphi, \partial \varphi, h$, and $A$, but one typically does not include a compensator scalar field $\phi$ (although $\phi$ is usually introduced in locally scale-invariant PGTs, see Section V, we will not consider this possibility here). Thus, the total Lagrangian density is

$$
\mathcal{L}_{\mathrm{T}}=\mathcal{L}_{\mathrm{G}}(h, \partial h, A, \partial A)+\mathcal{L}_{\mathrm{M}}(\varphi, \partial \varphi, h, A),
$$

where we have indicated the functional dependencies in the most general case. The general structure of the resulting equations of motion for the gauge fields is the same as that described for WGT in Section II H, except that the $B$-field equation (62c) in WGT is obviously absent in PGT. Similarly, the equation of motion for the matter field $\varphi$ has the same form as (67a) but with all asterisks removed, but the $\phi$-field equation (67b) in WGT is typically absent in PGT. The conservation laws in PGT may also be obtained from those given for WGT in Section II I, by removing all asterisks and setting $\mathcal{H}_{a b}, j^{a}, \zeta^{a}$ to zero and $\phi$ to unity in (68a)-(68b) and (69a)-(69b). Clearly, the WGT conservation laws (68c) and (69c) relating to local dilation invariance are usually absent in PGT.

The coupling of the PGT gravitational gauge fields to the Dirac matter field and electromagnetic field is analogous to that in WGT, discussed in Sections II J and II K, except that one does not typically introduce the scalar (compensator) field $\phi$. Indeed, as mentioned in these discussions, in both cases the Lagrangian density in PGT is identical to that in WGT, with the slight modification $\mu \phi \bar{\psi} \psi \rightarrow m \bar{\psi} \psi$ for the Dirac field. Thus, after this change and then following our prescription of removing all asterisks and setting $B_{\mu}=0$ and $\phi=1$, all the results in these sections are also valid in PGT. Similar considerations apply to results regarding the motion of test particles given in Section II M, with the key conclusion that they follow the gauge theory equivalent of geodesic motion, rather than autoparallels.

For "reduced" PGT, an analogous situation to that described in Section II N holds. In particular, the results regarding the construction of "reduced" field strengths also hold in PGT if one again sets $B_{\mu}=0$ (and so $H_{\mu v}=0$ also) and removes all asterisks. Since the reduced "translational" field strength of the $h$-field again vanishes identically, ${ }^{0} \mathcal{T}^{a}{ }_{b c} \equiv 0$, only the reduced "rotational" field strength ${ }^{0} \mathcal{R}^{a b}{ }_{c d}\left(h, \partial h, \partial^{2} h\right)$ remains, which depends only on the $h$-field and its first and second derivatives, as indicated. Thus, the entire resulting theory can be written purely in terms of just the $h$-gauge field. For the Bianchi identities involving "reduced" field strengths, it is worth noting that the PGT versions of (51) and (52) reduce to the familiar differential Bianchi identity and cyclic identity, respectively, of the Riemann tensor in $V_{4}$. Also, (55) and (56) reduce, respectively, to the familiar results in $V_{4}$ that the covariant divergence of the Einstein tensor vanishes and the Ricci tensor is symmetric. The expressions for how the "full" PGT rotational field strength tensor $\mathcal{R}^{a b}{ }_{c d}$ and its contractions are related to their "reduced" counterparts are obtained by removing all asterisks from (110a)-(110c). It is worth noting that the PGT version of the last term on the RHS of (110c) may be rewritten in terms of a total derivative as ${ }^{0} \mathcal{D}_{a} \mathcal{T}^{a}=h \partial_{\mu}\left(h^{-1} h_{a}{ }^{\mu} \mathcal{T}^{a}\right)$, whereas this is not possible in WGT. ${ }^{105}$

For reduced PGT, the free gravitational Lagrangian analogous to (B1) is

$$
L_{\mathrm{G}}=-\kappa^{-1}\left(\Lambda+a^{0} \mathcal{R}\right)+L_{0^{2}},
$$

where $L_{0^{2}}$ is based on (2) with $\mathcal{R}^{a b}{ }_{c d}$ replaced by ${ }^{0} \mathcal{R}^{a b}{ }_{c d}$. Unlike its WGT counterpart, however, ${ }^{0} \mathcal{R}^{a b}{ }_{c d}$ obeys all the usual symmetries of the curvature tensor in a Riemannian $V_{4}$ spacetime, and so the number of distinct terms in $L_{0_{\mathcal{R}^{2}}}$ is reduced to

$$
L_{0} \mathcal{R}^{2}=\alpha_{1}{ }^{0} \mathcal{R}^{2}+\alpha_{2}{ }^{0} \mathcal{R}_{a b}{ }^{0} \mathcal{R}^{a b}+\alpha_{3}{ }^{0} \mathcal{R}_{a b c d}{ }^{0} \mathcal{R}^{a b c d},
$$

where the $\alpha_{i}$ are dimensionless free parameters. In fact, one can simplify (B4) still further (in $D \leq 4$ dimensions), since ${ }^{0} \mathcal{R}_{a b c d}$ and its contractions also satisfy a Gauss-Bonnet identity of the form (59), but with $\mathcal{R}_{a b c d} \rightarrow{ }^{0} \mathcal{R}_{a b c d}$. Thus, one can set to zero any one of the parameters $\alpha_{i}$ in (B4) with no loss of generality (at least classically). The calculation of the corresponding field equations is simplest if one sets $\alpha_{3}=0$, which is the preferred choice, and thus yields the modest four-parameter family of free gravitational Lagrangians

$$
L_{\mathrm{G}}=-\kappa^{-1}\left(\Lambda+a^{0} \mathcal{R}\right)+\alpha_{1}{ }^{0} \mathcal{R}^{2}+\alpha_{2}{ }^{0} \mathcal{R}_{a b}{ }^{0} \mathcal{R}^{a b} .
$$


This Lagrangian depends only on the $h$ gauge field and its derivatives. In general, however, it is quadratic in second-order derivatives of the $h$-field, and so the corresponding field equations will typically be linear in fourth-order derivatives of $h$; such theories typically suffer from Ostrogradsky's instability, although this must be investigated on a case-by-case basis. If one demands the field equations to be at most second-order in derivatives of $h$, then one must set $\alpha_{1}=\alpha_{2}=0$ (at least in $D \leq 4$ spacetime dimensions), and on choosing $a=\frac{1}{2}$ the resulting theory corresponds precisely to general relativity with a cosmological constant, when interpreted geometrically. Another interesting choice of coefficients in (B5) is $a=0=\Lambda, \alpha_{1}=-\frac{1}{3} \alpha_{2}$, which corresponds to the free gravitational Lagrangian of so-called conformal gravity theory, when interpreted geometrically; ${ }^{101}$ this theory is discussed briefly in Section V B as the unique example of a locally scale-invariant reduced PGT.

Finally, the geometric interpretation of PGT follows from the corresponding discussion of WGT in Section II O by adopting the usual prescription of removing all asterisks and setting $B_{\mu}$ (and therefore $H_{\mu \nu}$ ) to zero. In particular, it is worth noting that RHS of the semi-metricity condition (124) vanishes in PGT, which shows that the spacetime has, in general, a Riemann-Cartan $U_{4}$ geometry, i.e., the connection is metric compatible but may exhibit non-zero torsion. Also, the quantities (125) and (126) become simply the curvature and (minus) torsion tensor of the $U_{4}$ spacetime, and (127) vanishes identically. For the geometric interpretation of the "reduced" quantities, one adopts the same approach as above, which leads one to recognise ${ }^{0} R^{\rho}{ }_{\sigma \mu \nu}$ as the standard curvature tensor in a Riemannian $V_{4}$ spacetime, which obeys all the familiar symmetries, plus the cyclic and Bianchi identities. It is worth noting that the comments made at the end of Section II O regarding the choice to interpret some quantities as geometric properties of the underlying spacetime and others as fields residing in that spacetime are equally true in PGT. As a concrete example, consider the PGT version of (110c) in the geometric interpretation, namely,

$$
R={ }^{0} R+\frac{1}{4} T^{\mu \nu \lambda} T_{\mu \nu \lambda}+\frac{1}{2} T^{\mu \nu \lambda} T_{\nu \mu \lambda}-T^{\mu} T_{\mu}-\frac{2}{\sqrt{-g}} \partial_{\mu}\left(\sqrt{-g} T^{\mu}\right)
$$

which relates the Ricci-Cartan scalar $R \equiv R^{\mu \nu}{ }_{\mu \nu}$ in $U_{4}$ spacetime to the Ricci scalar ${ }^{0} R \equiv{ }^{0} R^{\mu \nu}{ }_{\mu \nu}$ in $V_{4}$ spacetime. In standard Einstein-Cartan theory (see Appendix $C$ for its interpretation as a gauge theory in Minkowski spacetime), the gravitational Lagrangian is taken to be $L_{\mathrm{G}}=-R /(2 \kappa)$ and the theory is interpreted as a geometric theory in $U_{4}$ spacetime. One could, however, equally well rewrite the Lagrangian using (B6) and interpret the theory as a geometric theory in $V_{4}$ spacetime that includes a tensor field $T_{\mu \nu \sigma}$ (obeying the appropriate symmetries) defined on that spacetime, for which the Lagrangian is given by the terms containing $T_{\mu \nu \lambda}$ in (B6) (apart from the final one, which can be omitted since it contributes only a total derivative to the integrand of the action).

\section{APPENDIX C: EINSTEIN-CARTAN THEORY}

The precise version of PGT under consideration is determined by the form of the gravitational Lagrangian density $\mathcal{L}_{\mathrm{G}}$ and the matter Lagrangian density $\mathcal{L}_{\mathrm{M}}$. As an example, we here give a brief account of the original such theory, first considered by Kibble, in which $\mathcal{L}_{\mathrm{G}}$ is chosen to be proportional to the allowable covariant expression of lowest degree, namely, the linear invariant $\mathcal{R}=\mathcal{R}^{a b}{ }_{a b}$. Thus, in Kibble's original theory, one has

$$
\mathcal{L}_{\mathrm{G}}=-\frac{1}{2 \kappa} h^{-1} \mathcal{R}
$$

which corresponds to setting $a=\frac{1}{2}$ and $\Lambda=\alpha_{i}=\beta_{i}=0$ in (B1); the factor $\frac{1}{2}$ is introduced for later convenience. The resulting theory is known as Einstein-Cartan (EC) theory, ${ }^{106}$ which, when re-interpreted geometrically, is a direct generalisation of general relativity to include torsion sourced by the spin-angular-momentum (if any) of the matter field. We will first consider a general such matter field, but then specialise to the Dirac field. 


\section{Field equations}

The above choice for the gravitational Lagrangian density simplifies the general structure of the PGT gravitational field equations a little, since $\mathcal{L}_{\mathrm{G}}$ does not depend on derivatives of the $h$-field. In particular, one finds that the gravitational field equations read (after slight rearrangement)

$$
\begin{aligned}
\mathcal{R}^{a c}{ }_{b c}-\frac{1}{2} \delta_{b}^{a} \mathcal{R} & =\kappa h \tau^{a}{ }_{b}, \\
h b^{c}{ }_{\mu} D_{\nu}\left[h^{-1}\left(h_{a}{ }^{\mu} h_{b}{ }^{\nu}-h_{a}{ }^{\nu} h_{b}{ }^{\mu}\right)\right] & =2 \kappa h \sigma_{a b}{ }^{c},
\end{aligned}
$$

where the energy-momentum and spin-angular-momentum, respectively, associated with the matter Lagrangian $\mathcal{L}_{\mathrm{M}}$ provide the source terms on the right-hand sides of the equations.

The first field equation (C2) is written primarily in terms of the rotational gauge field strength $\mathcal{R}^{a b}{ }_{c d}$ and clearly resembles the Einstein equations in general relativity, although with a very different interpretation here. We can, in fact, rewrite the second field equation (C3) in terms of the translational field strength $\mathcal{T}^{a} b c$ to obtain

$$
\mathcal{T}^{c}{ }_{a b}+\delta_{a}^{c} \mathcal{T}_{b}-\delta_{b}^{c} \mathcal{T}_{a}=2 \kappa h \sigma_{a b}{ }^{c},
$$

where $\mathcal{T}_{a} \equiv \mathcal{T}^{c}{ }_{a c}$, as defined in (66). In particular, by contracting (C4) on the indices $b$ and $c$, we find

$$
\mathcal{T}_{a}=-\kappa h \sigma_{a b}{ }^{b} .
$$

This result may be used to re-write the second field equation (C4) to give an explicit expression for the translational gauge field strength, which reads

$$
\mathcal{T}^{c}{ }_{a b}=\kappa h\left(2 \sigma_{a b}{ }^{c}+\delta_{a}^{c} \sigma_{b d}{ }^{d}-\delta_{b}^{c} \sigma_{a d}{ }^{d}\right) .
$$

It is worth noting that this relationship is algebraic, rather than differential. Thus, as mentioned in the Introduction, the $\mathcal{T}$-field is non-propagating, i.e., it must vanish outside of spin sources.

It should be noted that the second field equation (C4) can, in principle, be solved for $A^{a b}{ }_{\mu}$ or, equivalently, for its fully Lorentz-index counterpart $\mathcal{A}^{a b}{ }_{c}=h_{c}{ }^{\mu} A^{a b}{ }_{\mu}$. In particular, combining the PGT form of the expression (46) (with all asterisks removed) and (C6), one quickly finds that

$$
\begin{aligned}
\mathcal{A}_{a b c}= & \frac{1}{2}\left(c_{a b c}+c_{b c a}-c_{c a b}\right) \\
& +\kappa h\left(\sigma_{a b c}-\sigma_{b c a}-\sigma_{c a b}+\eta_{a c} \sigma_{b d}{ }^{d}-\eta_{b c} \sigma_{a d}{ }^{d}\right),
\end{aligned}
$$

where $c^{c}{ }_{a b}$ was defined in (45) (again removing all asterisks). In the general case, in which $\sigma_{a b}{ }^{c} \neq 0$, this is not, however, an explicit solution since $\mathcal{A}_{a b c}$ also occurs on the right-hand side. Nonetheless, if $\mathcal{L}_{\mathrm{M}}$ is linear in the derivatives of the matter field(s), then $\sigma_{a b c}$ is independent of $\mathcal{A}_{a b c}$ and so (C7) is an explicit solution for $\mathcal{A}_{a b c}$. In this case, $\mathcal{A}_{a b c}$ is no longer an independent dynamical variable, but is derivable from the $h$-field, and so one may express the entire theory in "second-order" form.

The second field equation (C4) also allows for straightforward consideration of special cases in which $\sigma_{a b}{ }^{c}$ satisfies given constraints. From (C5), we see that in the special case where $\sigma_{a b}{ }^{b}=0$, then $\mathcal{T}_{a}$ also vanishes, thereby greatly simplifying (C4). Moreover, if the spin-angular-momentum of the matter field vanishes altogether, such that $\sigma_{a b}{ }^{c}=0$, then so too must the translational gauge field strength $\mathcal{T}^{c}{ }_{a b}$, and so the $h$-field itself must satisfy

$$
\mathcal{D}_{b} h_{a}{ }^{\mu}-\mathcal{D}_{a} h_{b}{ }^{\mu}=0 \text {. }
$$

\section{Dirac matter field}

It is worth noting that, if the matter source in EC theory is a massive Dirac field $\psi$, such that the matter Lagrangian density is given by (70) with $\mu \phi \equiv m$, several simplifications occur. First, the Dirac matter Lagrangian is linear in the derivatives of $\psi$, so that the spin-angular-momentum tensor $\sigma_{a b c}$ is independent of the rotational gauge field $\mathcal{A}_{a b c}$. Thus, as discussed above, the latter may be expressed in terms of the $h$-field, and is no longer an independent field. Moreover, from (C5), we 
find that

$$
\mathcal{T}_{a}=-\kappa h \sigma_{a b}{ }^{b}=0,
$$

since $\sigma_{a b c}=\sigma_{[a b c]}$ for Dirac matter and so all contractions of the spin-angular-momentum tensor vanish. An immediate consequence of (C9) is that the second gravitational field equation (C4) takes the much simpler form $\mathcal{T}^{c}{ }_{a b}=2 \kappa h \sigma_{a b}{ }^{c}$. More interestingly, the condition (C9) implies that the covariant Dirac equation reduces to

$$
i \gamma^{a} \mathcal{D}_{a} \psi-m \psi=0
$$

which is precisely the form that would be obtained by applying the minimal-coupling procedure directly at the level of the Dirac equation, rather than at the level of the action. Thus, we see that in EC theory, at least, the two approaches are consistent.

\section{Geometrical interpretation of EC theory}

Finally, we comment briefly on the geometric interpretation of the special case of Einstein-Cartan theory, discussed in Appendix C. In this case, the gravitational field equations (C2) and (C6) can be rewritten in the form

$$
\begin{aligned}
R_{\mu \nu}-\frac{1}{2} g_{\mu \nu} R & =\kappa \frac{\tau_{\mu \nu}}{\sqrt{-g}}, \\
T^{\lambda}{ }_{\mu \nu} & =\frac{\kappa}{2 \sqrt{-g}}\left(2 \sigma_{\mu \nu}{ }^{\lambda}+\delta_{\mu}^{\lambda} \sigma_{\nu \rho}{ }^{\rho}-\delta_{\nu}^{\lambda} \sigma_{\mu \rho}{ }^{\rho}\right),
\end{aligned}
$$

where $R_{\mu \nu} \equiv R_{\mu \lambda \nu}^{\lambda}$ and $R \equiv R_{\mu}^{\mu}$ have their usual forms, but one must remember that the affine connection $\Gamma_{\mu \nu}^{\lambda}$ is not, in general, symmetric in $\mu$ and $v$. Also, $\tau_{\mu \nu}=e^{a}{ }_{\mu} e^{b}{ }_{\nu} \tau_{a b}$ and $\sigma_{\mu \nu}{ }^{\lambda}=$ $e^{a}{ }_{\mu} e^{b}{ }_{\nu} e_{c}{ }^{\lambda} \sigma_{a b}{ }^{c}$, where the former is, in general, not symmetric.

It is worth noting that, in the case where the spin-angular-momentum $\sigma_{\mu \nu}{ }^{\lambda}$ of the matter field vanishes, then so too does the torsion $T^{\lambda}{ }_{\mu \nu}$ and hence the affine connection reduces to the metric connection. In this case, $R_{\mu \nu}$ reduces to the standard symmetric Ricci tensor ${ }^{0} R_{\mu \nu} \equiv R_{\mu \nu}\left(V_{4}\right)$ in a Riemann spacetime, expressed entirely in terms of the metric $g_{\mu \nu}$. Hence (C11) reduces to the usual form for the Einstein equation and so, in this limit, the geometrical interpretation of EC theory reduces to general relativity.

\section{APPENDIX D: DIRAC GRAVITATIONAL THEORY}

The precise form of WGT under consideration depends on the form of the free gravitational Lagrangian density $\mathcal{L}_{\mathrm{G}}$ and matter Lagrangian density $\mathcal{L}_{\mathrm{M}}$. As an illustration, we consider here (an extension of) the first scale-invariant theory of gravity to be proposed that can accommodate "ordinary" matter, which was explored by Dirac ${ }^{54}$ in the context of attempting to establish a deeper physical understanding of his "large numbers hypothesis" relating microscopic (quantum) and macroscopic (gravitational) scales. Dirac originally cast his theory in wholly geometrical terms, but it can be naturally considered in the framework of WGT in Minkowski spacetime, as we show below. ${ }^{58}$ Indeed, the WGT approach allows for a straightforward generalisation of Dirac's theory to non-zero torsion, which we will include ${ }^{50}$ (thus Dirac's original theory was, in fact, an example of a reduced WGT, as discussed in Section II N). Our discussion parallels that carried out by Lewis et al. ${ }^{107}$ in the language of geometric algebra, but is presented here in the context of our more general formulation of WGT.

\section{Free gravitational and matter actions}

In constructing the free gravitational Lagrangian density $\mathcal{L}_{\mathrm{G}}=h^{-1} L_{\mathrm{G}}$, the requirements of local Weyl symmetry restrict our choice of $L_{\mathrm{G}}$ to the general " $\mathcal{R}^{2}+\mathcal{H}^{2}$ "-form in (4). The WGT equivalent of the free gravitational Lagrangian suggested by Dirac has the simple form ${ }^{108}$

$$
L_{\mathrm{G}}=-\frac{1}{4} \xi \mathcal{H}_{a b} \mathcal{H}^{a b}
$$


in which no " $\mathcal{R}^{2}$ "-terms appear, and where $\xi$ is a dimensionless constant (usually positive); the factor of $-\frac{1}{4}$ is included for later convenience.

Turning to the matter action, in addition to some original matter field $\varphi$, Dirac also added a "compensator" massless scalar field $\phi$. In particular, he included a kinetic term for $\phi$, a selfinteraction term proportional to $\phi^{4}$, and a term proportional to $\phi^{2} \mathcal{R}$ that non-minimally (conformally) couples $\phi$ to the rotational gravitational field strength. Thus, one has

$$
\begin{aligned}
L_{\mathrm{M}} & \equiv L_{\varphi}+L_{\phi}+\phi^{2} L_{\mathcal{R}} \\
& =L_{\varphi}+\frac{1}{2} \nu \mathcal{D}_{a}^{*} \phi \mathcal{D}^{* a} \phi-\lambda \phi^{4}-\frac{1}{2} a \phi^{2} \mathcal{R},
\end{aligned}
$$

where $v, \lambda$, and $a$ are dimensionless constants (usually positive), and factors of $\frac{1}{2}$ are included for later convenience. The functional dependencies of the Lagrangian for the matter field $\varphi$ are $L_{\varphi}(\varphi, \partial \varphi, h, A, B, \phi)$; note that $L_{\varphi}$ depends on $\phi$ if it includes some interaction term between $\phi$ and $\varphi$. Indeed, Dirac provided the original motivation for such matter Lagrangians, varying forms of which we have adopted in Sections II J, V A, and V B. ${ }^{109}$

The total Lagrangian density is then $\mathcal{L}_{T}=h^{-1} L_{T}$, where $L_{T}$ has the form

$$
\begin{aligned}
L_{T} & =L_{\mathrm{M}}+L_{\mathrm{G}} \\
& =L_{\varphi}+\frac{1}{2} \nu \mathcal{D}_{a}^{*} \phi \mathcal{D}^{* a} \phi-\lambda \phi^{4}-\frac{1}{2} a \phi^{2} \mathcal{R}-\frac{1}{4} \xi \mathcal{H}_{a b} \mathcal{H}^{a b} .
\end{aligned}
$$

We note again that the Lagrangian $L_{\varphi}$ is allowed, in general, to contain the scalar field $\phi$, and that the total Lagrangian does not contain any derivatives of the $h$-field. It is also worth noting that all the coupling constants $(a, \xi, v, \lambda)$ are dimensionless (and usually positive). In the case of $a$, Dirac (and others) believed that this implied $\phi^{-2}$ should replace Newton's gravitational constant $G$. Hence, if $\phi$ changed with time, tied to cosmological expansion in some way, then the effective $G$ would also change with time, thereby allowing his "large numbers hypothesis" (see the Introduction) to remain true at all cosmic epochs. In addition, Dirac chose to fix some of the constants in the Lagrangian so that $L_{T}$ did not contain the dilation gauge field $B_{\mu}$ explicitly (except perhaps through $L_{\varphi}$ ). Here, we will instead retain the more general form (D3).

A more profound difference between our approach and that originally taken by Dirac is that we will consider the theory in the context of WGT in Minkowski spacetime, rather than as a metric-based geometrical theory, although the former is easily interpreted in geometric terms, as discussed in Section II O. Perhaps more importantly, we will obtain the corresponding field equations by varying the action (D3) with respect to the four independent fields $h_{a}{ }^{\mu}, A^{a b}{ }_{\mu}, B_{\mu}$, and $\phi$, respectively, ${ }^{50}$ whereas Dirac varied his geometrically interpreted action with respect to $B_{\mu}, \phi$, and the spacetime metric $g_{\mu \nu}$. Our approach is thus closer in spirit to the Palatini formalism in geometric theories, in which the metric and the connection are varied independently, as opposed to variation with respect to the metric alone, assuming a predetermined form of the connection. Moreover, as already mentioned, Dirac chose the latter approach with the connection assumed to have the form given in (131), so that torsion is zero by construction, whereas our approach naturally allows for non-zero torsion.

Unsurprisingly, our approach does lead to some differences in the final field equations as compared with those obtained by Dirac but, as we will show, these differences are, in fact, rather minor and the overall structure of the resulting theory is very similar using either approach.

\section{Field equations}

On varying the action (D3), the $h$-field equation is found to be

$$
a \phi^{2}\left(\mathcal{R}^{a}{ }_{b}-\frac{1}{2} \delta_{b}^{a} \mathcal{R}\right)=h\left(\tau_{\varphi}\right)_{b}^{a}+\left[v\left(\mathcal{D}^{* a} \phi\right)\left(\mathcal{D}_{b}^{*} \phi\right)-\delta_{b}^{a} L_{\phi}\right]-\xi\left(\mathcal{H}^{a c} \mathcal{H}_{b c}-\frac{1}{4} \delta_{b}^{a} \mathcal{H}_{c d} \mathcal{H}^{c d}\right),
$$

where $\left(\tau_{\varphi}\right)_{b}^{a}$ is the energy-momentum tensor of the matter field $\varphi$, and we recognise the second and third terms on the RHS as the energy-momentum tensors of the scalar field $\phi$ and of the dilation gauge field $B_{\mu}$, respectively. 
The $A$-field equation is given by

$$
a\left[\phi^{2} \mathcal{T}_{a b}^{* c}+\delta_{a}^{c}\left(\mathcal{D}_{b}^{*}+\mathcal{T}_{b}^{*}\right) \phi^{2}-\delta_{b}^{c}\left(\mathcal{D}_{a}^{*}+\mathcal{T}_{a}^{*}\right) \phi^{2}\right]=2 h\left(\sigma_{\varphi}\right)_{a b}^{c},
$$

where $\left(\sigma_{\varphi}\right)_{a b}{ }^{c}$ is the spin-angular-momentum tensor of the $\varphi$ matter field. It is worth noting that (D5) still holds if one makes the replacements $\mathcal{D}_{a}^{*} \rightarrow \mathcal{D}_{a}, \mathcal{T}^{* c}{ }_{a b} \rightarrow \mathcal{T}^{c}{ }_{a b}$, and $\mathcal{T}_{a}^{*} \rightarrow \mathcal{T}_{a}$ to PGT quantities, although the resulting equation is clearly no longer manifestly WGT covariant.

Varying the action (D3) with respect to the $B$-field, and rewriting the resulting equation of motion in manifestly WGT covariant form, one finds

$$
\xi\left[\frac{1}{2} \mathcal{T}^{* c}{ }_{a b} \mathcal{H}^{a b}-\left(\mathcal{D}_{a}^{*}+\mathcal{T}_{a}^{*}\right) \mathcal{H}^{a c}\right]-v \phi \mathcal{D}^{* c} \phi=h\left(\zeta_{\varphi}\right)^{c},
$$

where $\left(\zeta_{\varphi}\right)^{c}$ is the dilation current of the $\varphi$ matter field. It is worth noting that the term proportional to $\xi$ can be rewritten in terms of PGT quantities by making the replacements $\mathcal{D}_{a}^{*} \rightarrow \mathcal{D}_{a}$, $\mathcal{T}^{* c}{ }_{a b} \rightarrow \mathcal{T}^{c}{ }_{a b}$, and $\mathcal{T}_{a}^{*} \rightarrow \mathcal{T}_{a}$.

Finally, the $\phi$-field equation has the form

$$
\frac{\partial L_{\varphi}}{\partial \phi}-v\left(\mathcal{D}_{a}^{*}+\mathcal{T}_{a}^{*}\right) \mathcal{D}^{* a} \phi-4 \lambda \phi^{3}-a \phi \mathcal{R}=0 .
$$

\section{Einstein gauge}

As discussed in Section II L in the context of WGT in general, provided $\phi \neq 0$, the set of field equations can be simplified considerably by recasting the theory entirely in terms of scale-invariant field variables defined in (91). As we showed, however, the resulting field equations in the new variables are identical in form to those in the original variables after setting $\phi(x)=\phi_{0}$, which is the standard Einstein gauge condition. Since the latter approach is more familiar and straightforward, we will adopt it here, but bear in mind the above equivalence throughout. It is worth noting that this conclusion precludes Dirac's original hope of substantiating a time-varying gravitational parameter $G$ and his large numbers hypothesis. To obtain the forms of the field equations (D4)-(D7) in the Einstein gauge, one merely sets $\phi=\phi_{0}$ and makes the replacement $\left.\mathcal{D}_{a}^{*} \phi\right|_{\phi=\phi_{0}}=-\phi_{0} \mathcal{B}_{a}$ throughout. It is also convenient to set $a=1 /\left(\kappa \phi_{0}^{2}\right)$, where $\kappa$ is Einstein's gravitational constant, which is a dimensionally consistent identification.

In this case, it is straightforward to show that the $h$-field equation (D4) becomes

$$
\mathcal{R}^{a}{ }_{b}-\frac{1}{2} \delta_{b}^{a} \mathcal{R}-\delta_{b}^{a} \Lambda=\kappa\left[\left.h\left(\tau_{\varphi}\right)^{a}{ }_{b}\right|_{\phi=\phi_{0}}+v\left(\mathcal{B}^{a} \mathcal{B}_{b}-\frac{1}{2} \delta_{b}^{a} \mathcal{B}^{c} \mathcal{B}_{c}\right)-\xi\left(\mathcal{H}^{a c} \mathcal{H}_{b c}-\frac{1}{4} \delta_{b}^{a} \mathcal{H}_{c d} \mathcal{H}^{c d}\right)\right],
$$

where $\Lambda=\lambda \phi_{0}^{2} / a=\kappa \lambda$ has the correct dimensions of (length) ${ }^{-2}$ for a physical cosmological constant. Thus, one recovers the $h$-field equation (C2) from standard EC theory, with the addition of an extra term on the LHS representing a cosmological constant, and extra terms on the RHS denoting the energy-momentum of the dilation gauge field $B_{\mu}$, which we recognise as that of a massive vector field (or Proca field) of mass-squared $m^{2}=v \phi_{0}^{2} / \xi$.

In the Einstein gauge, the $A$-field equation (D5) becomes

$$
\mathcal{T}^{c}{ }_{a b}+\delta_{a}^{c} \mathcal{T}_{b}-\delta_{b}^{c} \mathcal{T}_{a}=\left.2 \kappa h\left(\sigma_{\varphi}\right)_{a b}{ }^{c}\right|_{\phi=\phi_{0}},
$$

where we have written the result in terms of PGT (unstarred) quantities for later convenience. This equation is identical to the form (C3) in EC theory. Thus, on imposing the Einstein gauge, we find that the Dirac gravitational theory reduces simply to EC theory, but with the addition of a cosmological constant and the massive vector field $B_{\mu}$. Since the field equation (D9) is algebraic, rather than differential, we must again conclude that, as in EC theory, the torsion is non-propagating; this arises from the free gravitational Lagrangian (D1) not containing any " $\mathcal{R}$ " " terms.

Adopting the Einstein gauge, the equation of motion (D6) for the $B$-field reads

$$
\xi\left[\frac{1}{2} \mathcal{T}^{c}{ }_{a b} \mathcal{H}^{a b}-\left(\mathcal{D}_{a}+\mathcal{T}_{a}\right) \mathcal{H}^{a c}\right]+v \phi_{0}^{2} \mathcal{B}^{c}=\left.h\left(\zeta_{\varphi}\right)^{c}\right|_{\phi=\phi_{0}},
$$

where we have again rewritten the result in terms of unstarred PGT quantities. 
Finally, the $\phi$-field equation (D7) becomes

$$
\mathcal{R}+4 \Lambda=\kappa\left[\left.\phi_{0} \partial_{\phi} L_{\varphi}\right|_{\phi=\phi_{0}}+v \phi_{0}^{2}\left(\mathcal{D}_{a}+\mathcal{T}_{a}+\mathcal{B}_{a}\right) \mathcal{B}^{a}\right],
$$

where we have once again written the result in terms of unstarred PGT quantities.

It is worth re-iterating at this point that, when written in terms of the scale-invariant variables defined in (91), the full field equations (D4)-(D7) have precisely the same form as those in (D8)-(D11) obtained in the Einstein gauge, after replacing (in the latter set) the matter field $\varphi$ and the gravitational gauge fields $h_{a}{ }^{\mu}, A^{a b}{ }_{\mu}$, and $B_{\mu}$ (and hence the PGT covariant derivative and the field strength tensors $\mathcal{R}^{a b}{ }_{c d}, \mathcal{T}^{a}{ }_{b c}=\mathcal{T}^{* a}{ }_{b c}-\delta_{c}^{a} \mathcal{B}_{b}+\delta_{b}^{a} \mathcal{B}_{c}$ and $\mathcal{H}_{a b}$ and their contractions) with their scale-invariant counterparts (notated with carets) discussed in Section II L.

\section{Dirac matter field}

Let us now consider the particular case where the matter is described by a Dirac field. Following the discussion in Section II J, an appropriate locally Weyl covariant Lagrangian for the Dirac field is given by

$$
L_{\psi}=\frac{1}{2} i \bar{\psi} \gamma \gamma^{a} \stackrel{\leftrightarrow}{\mathcal{D}_{a}} \psi-\mu \phi \bar{\psi} \psi,
$$

where $\mu$ is a dimensionless parameter. It is worth noting that $\mu \phi$ has the dimensions of mass in natural units.

The energy-momentum and spin-angular-momentum tensors of the Dirac field $\psi$ are again given by (77) and (78), respectively, but with $m$ replaced by $\mu \phi$. Since $B_{\mu}$ does not appear in (D12), the dilation current $\left(\zeta_{\psi}\right)^{\mu}=0$ for the Dirac field. Moreover, since the $A$-field equation (D9) in the Einstein gauge is identical to $(\mathrm{C} 4)$ in EC theory, from $(\mathrm{C} 10)$, we again have $\mathcal{T}_{a}=0$. Thus, the equation of motion for the Dirac field is simply

$$
i \gamma^{a} \mathcal{D}_{a} \psi-\mu \phi \psi=0
$$

which, in turn, ensures that $L_{\mathrm{M}}$ evaluates to zero on shell. As a result, the trace of the energymomentum tensor of the Dirac field is easily found to be $\tau_{\psi}=h^{-1} \mu \phi \bar{\psi} \psi$. Applying the Einstein gauge condition $\phi=\phi_{0}$ to the above results is trivial.

\section{Dilation gauge field as a dark matter candidate}

In the Einstein gauge, both the $h$-field and $A$-field equations are identical to those in standard EC theory, with the addition of a cosmological constant and the dilation gauge field. It is therefore worth exploring the $B$-field equation in more detail to determine what additional physics beyond EC theory (if any) is contained in Dirac gravitational theory sourced by Dirac matter. Expanding out the field strengths on the LHS of (D10) in terms of the $B$-field, swapping the order of some covariant derivatives, and employing the results (C5), (C6), and (C9) from EC theory with Dirac matter, after a long calculation one obtains

$$
\mathcal{D}_{a} \mathcal{D}^{a} \mathcal{B}^{c}-\mathcal{R}^{a c} \mathcal{B}_{a}+m^{2} \mathcal{B}^{c}+2 \kappa\left[\left(\delta_{b}^{c} \mathcal{D}_{a}-\kappa h \sigma_{a b}{ }^{c}\right)\left(h \sigma^{a b d} \mathcal{B}_{d}\right)\right]=0,
$$

where we have defined $m^{2} \equiv v \phi_{0}^{2} / \xi$ and $\sigma_{a b}{ }^{c}$ is the spin-angular-momentum tensor of the Dirac field (evaluated in the Einstein gauge). Thus we see that the massive vector field $\mathcal{B}_{a}$ has a nonminimal coupling to the gravitational field and only couples to the Dirac matter field through its spin-angular-momentum. Moreover, in modelling a realistic matter fluid composed of spin-1/2 particles, one would expect spin-averaging effects to result in the spin-angular-momentum that the Dirac field would otherwise represent being massively diluted. Thus, we expect the vector field to be only weakly interacting with ordinary matter and it hence makes an interesting dark matter candidate. Clearly, in the case where the (effective) spin-angular-momentum of the matter field vanishes (and thus so does the torsion), then (D14) reduces to the equation of motion for a free massive vector field of mass $m$ non-minimally coupled to the gravitational field. 
In closing, it is worth pointing out that, in addition to automatically containing a candidate for dark matter, Dirac gravitational theory also provides a natural means for introducing a cosmological constant term in the gravitational field equation (D8). Thus, it would seem worthwhile to explore its phenomenology further, in particular its cosmological consequences.

\section{APPENDIX E: ALTERNATIVE DERIVATION OF EWGT COVARIANT DERIVATIVE}

We present here an alternative approach to deriving the form of the covariant derivative (143) in eWGT, which is complementary to that presented in Section III A and was, in fact, the original route taken by the authors. In particular, in this approach the Weyl weight $w$ of the field on which the covariant derivative acts does not need to be inserted "by hand," but instead arises naturally.

One begins by noting that in the standard WGT covariant derivative (20), namely, $D_{\mu}^{*}=$ $D_{\mu}+w B_{\mu}$, the value of $w$ has to change depending on the Weyl weight of the field on which it acts and this leads to the commutator of two such derivatives being similarly non-unique. This might all be deemed somewhat displeasing. Thus, as an alternative, one might instead consider introducing a vector dilation gauge field (called $V_{\mu}$ to distinguish it from the field $B_{\mu}$ in WGT) in a different manner, by including it in a modified rotational gauge field of the form (142), namely,

$$
A_{\mu}^{\dagger a b}=A_{\mu}^{a b}+\left(\mathcal{V}^{a} b^{b}{ }_{\mu}-\mathcal{V}^{b} b^{a}{ }_{\mu}\right),
$$

where $\mathcal{V}_{a}=h_{a}{ }^{\mu} V_{\mu}$. In turn, this leads one to introduce the new derivative operators

$$
\mathcal{D}_{a}^{\natural} \varphi \equiv h_{a}{ }^{\mu} D_{\mu}^{\natural} \varphi \equiv h_{a}{ }^{\mu}\left(\partial_{\mu}+\frac{1}{2} A^{\dagger b c}{ }_{\mu} \Sigma_{b c}\right) \varphi .
$$

As mentioned at the start of Section III, the PGT matter actions for the massless Dirac field and the electromagnetic field are already invariant under local dilations with the extended transformation law (137) for the $A$-field, in the same way as they are for the normal transformation law (135). Indeed, this provides a strong motivation for considering the extended form. This does mean, however, that one cannot determine the transformation law under local dilations of the gauge field $V_{\mu}$, and hence either derivative operator in (E2), by the usual technique of replacing the PGT covariant derivative $\mathcal{D}_{a}$ with $\mathcal{D}_{a}^{\natural}$ in either the Dirac or electromagnetic field action, and demanding invariance under local dilations.

As an alternative, however, one can fix the transformation properties of $V_{\mu}$ by demanding instead that the field strength tensor derived from the commutator of two derivatives transforms covariantly under local dilations. One finds that the commutator is given by

$$
\left[D_{\mu}^{\natural}, D_{\nu}^{\natural}\right] \varphi=\frac{1}{2} R^{\dagger a b}{ }_{\mu \nu} \Sigma_{a b} \varphi,
$$

where $R^{\dagger a b}{ }_{\mu \nu}$ is the eWGT rotational gauge field strength found previously and given in (151a). Then, demanding that $R^{\dagger a b}{ }_{\mu \nu}$ transforms covariantly under local dilations with the extended transformation law for the $A$-field, one requires that, as found previously, the dilation gauge field transforms as

$$
V_{\mu}^{\prime}=V_{\mu}+\theta P_{\mu},
$$

where $P_{\mu} \equiv \partial_{\mu} \rho$. This transformation for $V_{\mu}$ implies that the modified rotational gauge field $A^{\dagger a b}{ }_{\mu}$ in (E1) and hence the field strength tensor $R^{\dagger a b}{ }_{\mu \nu}$ are both invariant $(w=0)$ under extended local dilations. One can then define $\mathcal{R}^{\dagger a b}{ }_{c d} \equiv h_{c}{ }^{\mu} h_{d}{ }^{v} R^{\dagger a b}{ }_{\mu \nu}$ as before, which transforms covariantly with weight $w=-2$.

By adopting the transformation law (E4) for $V_{\mu}$, however, one quickly finds that $D_{\mu}^{\natural} \varphi$ does not transform covariantly under extended local dilations, hence explaining our reluctance thus far to call $D_{\mu}^{\natural}$ (or $\mathcal{D}_{a}^{\natural}$ ) a covariant derivative. Nonetheless, using the covariant field strength tensor $\mathcal{R}_{a b c d}^{\dagger}$ that we have constructed, one can still define a new scale-invariant theory of gravity identical to that presented in Section IV, but with $\xi=0$ in the free gravitational Lagrangian $L_{\mathrm{G}}$ and $v=\beta_{1}=\beta_{2}=0$ in the matter Lagrangian $L_{\mathrm{M}}$, since we have not (yet) defined the quantities appearing in these terms. By varying the resulting total Lagrangian with respect to the gauge fields $h_{a}{ }^{\mu}$ and $A^{a b}{ }_{\mu}$ (and also $V_{\mu}$ and $\phi$, if desired, although this does not yield independent field equations), one obtains precisely 
the same equations of motion as those presented in Section IV A, but with $\xi=v=\beta_{1}=\beta_{2}=0$. These equations of motion are covariant under extended local dilations, despite $\mathcal{D}_{a}^{\natural}$ not being a covariant derivative. Indeed, for any covariant field $\chi$ (which may be either a matter field $\varphi$ or $\phi$, or the rotational gauge field strength tensor $\mathcal{R}_{a b c d}^{\dagger}$, inspection of the equations of motion allows one to identify the terms that need to be added to $\mathcal{D}_{a}^{\natural} \chi$ to assemble the appropriate covariant derivative $\mathcal{D}_{a}^{\dagger} \chi$. In each case, one finds that $\mathcal{D}_{a}^{\dagger} \chi=h_{a}{ }^{\mu} D_{\mu}^{\dagger} \chi$, where $D_{\mu}^{\dagger}$ has the form (143), but with the appropriate value of the Weyl weight $w$ of the field $\chi$ picked out automatically.

Similarly, inspection of the equations of motion allows one to identify the covariant object $\mathcal{T}_{a b c}^{\dagger}$. Indeed, once one has picked out the appropriate form of the eWGT covariant derivative operator $\mathcal{D}_{a}^{\dagger}$, one can then consider its commutator (153) and identify $\mathcal{T}_{a b c}^{\dagger}$ and also $\mathcal{H}_{a b}^{\dagger}$ as further gauge field strength tensors, as introduced in Section III D. These can then be added to the total Lagrangian, as before, to arrive back at the full scale-invariant gauge theory of gravity discussed in Section IV, without requiring $\xi=v=\beta_{1}=\beta_{2}=0$.

\section{APPENDIX F: SYMBOLS AND NOTATION}

Since this paper contains a considerable amount of special notation, for readers' convenience we summarise in Table I those symbols that occur most frequently.

TABLE I. Frequently occurring symbols and notation used in this paper. We list only those quantities relating to the Minkowski spacetime interpretation of the gauge field theories; for a discussion of their geometric interpretation in terms of more general spacetimes and the associated notation, see Sections II O and III P and Appendix B. See also the discussion of the index conversion properties of $h_{a}{ }^{\mu}$ and $b^{a}{ }_{\mu}$ in Section II C, and the associated notation; aside from the gauge fields themselves, in this table we list primarily the covariant versions of quantities, which have exclusively Latin indices. Note that scale-invariant (Weyl weight $w=0$ ) versions of the quantities listed below are denoted with a caret, for example, $\widehat{h}_{a}^{\mu}$ or $\widehat{\mathcal{D}}_{a}^{*}$ or $\widehat{\mathcal{R}}_{c d}^{a b}$; see Sections II L and III M.

\begin{tabular}{|c|c|}
\hline$h_{a}{ }^{\mu}$ & Translational gauge field ( $h$-field) \\
\hline$h$ & Determinant of $h_{a}{ }^{\mu}$ \\
\hline$b^{a}{ }_{\mu}$ & Inverse $h$-field \\
\hline$A^{a b}{ }_{\mu}$ & Rotational gauge field ( $A$-field) \\
\hline$B_{\mu}$ & WGT dilational gauge field ( $B$-field) \\
\hline$V_{\mu}$ & eWGT dilational gauge field ( $V$-field) \\
\hline$A_{\mu}^{\dagger a b} \equiv A_{\mu}^{a b}+2 \eta^{c[a} b^{b]}{ }_{\mu} h_{c}^{\nu} V_{\nu}$ & eWGT extended rotational gauge field ( $A^{\dagger}$-field) \\
\hline \multicolumn{2}{|l|}{ Derivative operators } \\
\hline $\mathcal{D}_{a} \equiv h_{a}{ }^{\mu} D_{\mu} \equiv h_{a}{ }^{\mu}\left(\partial_{\mu}+\frac{1}{2} A^{a b}{ }_{\mu} \Sigma_{a b}\right)$ & PGT (generalised) covariant derivative ${ }^{\mathrm{a}}$ \\
\hline$\partial_{\mu}^{*} \equiv \partial_{\mu}+w B_{\mu}$ & WGT “augmented" partial derivative \\
\hline $\mathcal{D}_{a}^{*} \equiv h_{a}{ }^{\mu} D_{\mu}^{*} \equiv h_{a}{ }^{\mu}\left(\partial_{\mu}^{*}+\frac{1}{2} A^{a b}{ }_{\mu} \Sigma_{a b}\right)$ & WGT (generalised) covariant derivative \\
\hline$\partial_{\mu}^{\dagger} \equiv \partial_{\mu}-w\left(V_{\mu}+\frac{1}{3} T_{\mu}\right)$ & eWGT “augmented” partial derivative \\
\hline $\mathcal{D}_{a}^{\dagger} \equiv h_{a}{ }^{\mu} D_{\mu}^{\dagger} \equiv h_{a}{ }^{\mu}\left(\partial_{\mu}^{\dagger}+\frac{1}{2} A^{\dagger a b}{ }_{\mu} \Sigma_{a b}\right)$ & eWGT (generalised) covariant derivative ${ }^{a}$ \\
\hline $\mathcal{D}_{a}^{\natural} \equiv h_{a}{ }^{\mu} D_{\mu}^{\natural} \equiv h_{a}^{\mu}\left(\partial_{\mu}+\frac{1}{2} A^{\dagger a b}{ }_{\mu} \Sigma_{a b}\right)$ & eWGT (generalised) semi-covariant derivative ${ }^{a}$ \\
\hline \multicolumn{2}{|l|}{ Gauge field strengths } \\
\hline $\mathcal{R}^{a b}{ }_{c d}=\mathcal{R}^{* a b}{ }_{c d}=$ & PGT and WGT rotational gauge field strength ${ }^{\mathrm{a}, \mathrm{b}}$ \\
\hline \multicolumn{2}{|l|}{$2 h_{a}{ }^{\mu} h_{b}{ }^{v}\left(\partial_{[\mu} A^{a b}{ }_{\nu]}+A^{a}{ }_{c[\mu} A^{c b}{ }_{v]}\right)$} \\
\hline $\mathcal{R}^{\dagger a b_{c d}}=2 h_{a}{ }^{\mu} h_{b}{ }^{v}\left(\partial_{[\mu} A^{\dagger a b}{ }_{\nu]}+A^{\dagger a}{ }_{c[\mu} A^{\dagger c b_{\nu]}}\right)$ & eWGT rotational gauge field strength ${ }^{\mathrm{a}, \mathrm{b}}$ \\
\hline $\mathcal{T}^{a}{ }_{b c}=2 h_{b}{ }^{\mu} h_{c}{ }^{v} D_{[\mu} b^{a}{ }_{v]}$ & PGT translational gauge field strength ${ }^{\mathrm{b}}$ \\
\hline $\mathcal{T}^{* a}{ }_{b c}=2 h_{b}{ }^{\mu} h_{c}{ }^{v} D_{[\mu}^{*} b^{a}{ }_{v]}$ & WGT translational gauge field strength ${ }^{\mathrm{b}}$ \\
\hline $\mathcal{T}^{\dagger a_{b c}}=2 h_{b}{ }^{\mu} h_{c}{ }^{v} D_{[\mu}^{\dagger} b^{a}{ }_{\nu]}$ & eWGT translational gauge field strength ${ }^{\mathrm{b}}$ \\
\hline $\mathcal{T}^{\natural a}{ }_{b c}=2 h_{b}{ }^{\mu} h_{c}{ }^{v} D_{[\mu}^{\natural} b^{a}{ }_{v]}$ & eWGT translational gauge "semi" field strength ${ }^{\mathrm{a}, \mathrm{b}}$ \\
\hline $\mathcal{H}_{a b}=2 h_{a}{ }^{\mu} h_{b}{ }^{v} \partial_{[\mu} B_{v]}$ & WGT dilational gauge field strength \\
\hline $\mathcal{H}_{a b}^{\dagger}=2 h_{a}{ }^{\mu} h_{b}{ }^{v} \partial_{[\mu}\left(V_{v}+\frac{1}{3} T_{v]}\right)$ & eWGT dilational gauge field strength \\
\hline
\end{tabular}


Table I. (Continued.)

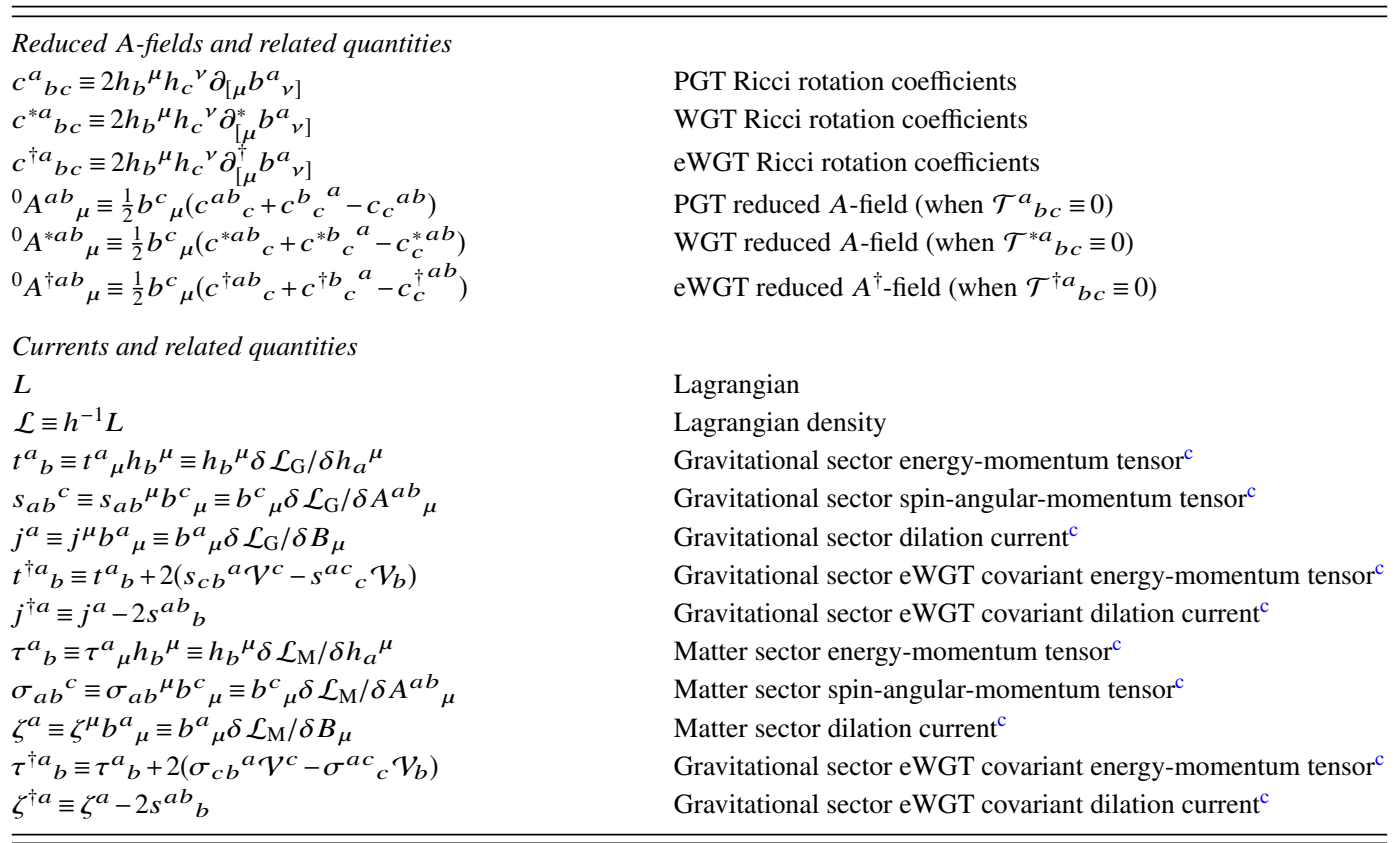

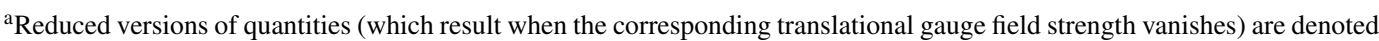
by a preceding zero superscript, for example, ${ }^{0} \mathcal{D}_{a}$ or ${ }^{0} \mathcal{R}^{a b}{ }_{c d}$, and are obtained by replacing $A^{a b}{ }_{\mu}$ or $A^{\dagger a b}{ }_{\mu}$ by the corresponding reduced $A$-field or $A^{\dagger}$-field, respectively; see Sections II N and III O and Appendix B.

${ }^{\mathrm{b}}$ Contractions are defined by $\mathcal{R}^{a}{ }_{b} \equiv \mathcal{R}^{a c}{ }_{b c}, \mathcal{R} \equiv \mathcal{R}^{a}{ }_{a}$, and $\mathcal{T}_{a}=\mathcal{T}^{b}{ }_{a b}$, and similarly for other versions.

${ }^{\mathrm{c}}$ Analogous quantities can be defined separately for the individual (covariant) parts of the gravitational and matter Lagrangian densities.

${ }^{1}$ R. Utiyama, Phys. Rev. 101, 1597 (1956).

2 T. W. B. Kibble, J. Math. Phys. 2, 212 (1961).

${ }^{3}$ D. Sciama, Rev. Mod. Phys. 36, 463 (1964).

${ }^{4}$ Note that, while $h_{a}{ }^{\mu}$ and $A^{a b}{ }_{\mu}$ are relatively standard notations for the translational and rotational gravitational gauge fields, respectively, the choice of notation for their corresponding field strength tensors varies considerably between authors; we have adopted a notation that anticipates the geometrical interpretation of these tensors as the curvature and (minus) torsion, respectively, of a Riemann-Cartan $\left(U_{4}\right)$ spacetime.

${ }^{5}$ F. W. Hehl, P. von der Heyde, G. D. Kerlick, and J. M. Nester, Rev. Mod. Phys. 48, 393 (1976).

${ }^{6}$ C. Wiesendanger, Classical Quantum Gravity 13, 681 (1996).

${ }^{7}$ If one varies only the fields and not the coordinates, as in the derivation of the Euler-Lagrange equations resulting from a given action, for example, then the "total" variation $\delta \varphi$ and the "form" variation $\delta_{0} \varphi$ clearly coincide, and is usually denoted by the former.

${ }^{8}$ N. Mukunda, in Gravitation, Gauge Theories and the Early Universe, edited by B. R. Iyer, N. Mukunda, and C. V. Vishveshwara (Kluwer, Dordrecht, 1989), p. 467.

${ }^{9}$ A. N. Lasenby, C. Doran, and S. F. Gull, Phil. Trans. R. Soc. Lond. A 356, 487 (1998).

${ }^{10}$ M. Blagojevic, Gravitation and Gauge Symmetries (IOP Publishing, Bristol, 2002).

${ }^{11}$ K. Hayashi and T. Shirafuji, Prog. Theor. Phys. 64, 866 (1980).

12 Y. N. Obukhov, V. N. Ponomariev, and V. V. Zhytnikov, Gen. Relativ. Gravitation 21, 1107 (1989).

${ }^{13}$ We adopt the following sign conventions: $(+,-,-,-)$ metric signature, $\left[\mathcal{D}_{c}, \mathcal{D}_{d}\right] \varphi \equiv \frac{1}{2} \mathcal{R}^{a b}{ }_{c d} \Sigma_{c d} \varphi-\mathcal{T}^{a}{ }_{c d} \mathcal{D}_{a} \varphi$, $\mathcal{R}^{a}{ }_{b} \equiv \mathcal{R}^{a c}{ }_{b c}$ and $\mathcal{T}_{a} \equiv \mathcal{T}^{b}{ }_{a b}$.

${ }^{14}$ P. Baekler and F. W. Hehl, Classical Quantum Gravity 28, 215017 (2011).

${ }^{15}$ Y. N. Obukhov and F. W. Hehl, Phys. Lett. B 713, 321 (2012).

${ }^{16}$ G. K. Karananas, Classical Quantum Gravity 32, 055012 (2015).

${ }^{17}$ F. W. Hehl, in Cosmology and Gravitation, edited by P. G. Bergmann and V. de Sabbata (Plenum, New York, 1980), p. 5.

${ }^{18}$ R. Kuhfuss and J. Nitsch, Gen. Relativ. Gravitation 18, 1207 (1986).

${ }^{19}$ R. P. Woodard, Scholarpedia 10, 32243 (2015).

${ }^{20}$ H. Motohashi and T. Suyama, Phys. Rev. D 91, 085009 (2015).

${ }^{21}$ P. von der Heyde, Phys. Lett. A 58, 141 (1976).

${ }^{22}$ A. Minkevich, Phys. Lett. A 80, 232 (1980).

${ }^{23}$ G. Rubilar, Classical Quantum Gravity 15, 239 (1998).

${ }^{24}$ R. T. Hammond, Rep. Prog. Phys. 65, 599 (2002).

${ }^{25}$ M. O. Katanaev, Gen. Relativ. Gravitation 25, 349 (1993).

${ }^{26}$ R. D. Hecht, J. Lemke, and R. R. Wallner, Phys. Rev. D 44, 2442 (1991). 
${ }^{27}$ R. D. Hecht, J. M. Nester, and V. V. Zhytnikov, Phys. Lett. A 222, 37 (1996).

${ }^{28}$ R. J. Rivers, Nuovo Cimento 34, 386 (1964).

${ }^{29}$ D. Neville, Phys. Rev. D 18, 3535 (1978).

${ }^{30}$ S. Weinberg, Phys. Rev. B 133, 1318 (1964).

${ }^{31}$ E. Sezgin and P. van Nieuwenhuisen, Phys. Rev. D 21, 3269 (1980).

${ }^{32}$ E. Sezgin, Phys. Rev. D 24, 1677 (1981).

${ }^{33}$ R. Battiti and M. Toller, Lett. Nuovo Cimento 44, 35 (1985).

${ }^{34}$ C. Rovelli, Nuovo Cimento 78, 167 (1983).

${ }^{35}$ P. van Nieuwenhuisen, Nucl. Phys. B 60, 478 (1973).

${ }^{36}$ A. Pais and G. E. Uhlenback, Phys. Rev 79, 145 (1950).

${ }^{37}$ D. Neville, Phys. Rev. D 21, 867 (1980).

${ }^{38}$ K. S. Stelle, Phys. Rev. D 16, 953 (1977).

${ }^{39}$ M. Y. Kalmykov and P. I. Pronin, Gen. Relativ. Gravitation 27, 873 (1995).

${ }^{40}$ G. Grignani and G. Nardelli, Phys. Rev. D 45, 2719 (1992).

${ }^{41}$ G. Grignani and G. Nardelli, Phys. Rev. D 48, 5032 (1993).

${ }^{42}$ T. Strobl, Phys. Rev. D 48, 5029 (1993).

${ }^{43}$ M. Y. Kalmykov, Classical Quantum Gravity 14, 367 (1997).

${ }^{44}$ T. Biswas, E. Gerwick, T. Koivisto, and A. Mazumdar, Phys. Rev. Lett. 108, 031101 (2012).

${ }^{45}$ T. Biswas, A. Conroy, A. S. Koshelev, and A. Mazumdar, Classical Quantum Gravity 31, 015022 (2014).

${ }^{46}$ L. Modesto and L. Rachwal, Nucl. Phys. B 900, 147 (2015).

${ }^{47}$ G. t'Hooft, Int. J. Mod. Phys. D 24, 1543001 (2015).

${ }^{48}$ A. Bregman, Prog. Theor. Phys. 49, 667 (1973).

49 J. Charap and W. Tait, Proc. R. Soc. A 340, 249 (1974).

${ }^{50}$ M. Kasuya, Nuovo Cimento B 28, 127 (1975).

${ }^{51}$ In his original paper, ${ }^{52}$ Weyl attempted to interpret the new gauge field $B_{\mu}$ as the electromagnetic 4-potential and thereby provide a unified description of gravity and electromagnetism. As Einstein later showed, however, the field $B_{\mu}$ interacts in the same manner with both particles and antiparticles, contrary to all experimental evidence about electromagnetic interactions. It was only later realised ${ }^{53}$ that electromagnetism was related to localisation of invariance under change of quantum-mechanical phase and, much later, that $\boldsymbol{B}_{\boldsymbol{\mu}}$ might be interpreted as an additional gravitational interaction.

52 H. Weyl, Sitzungsber. Preuss. Akad. Wiss. (Berlin) 26, 465 (1918).

${ }^{53}$ H. Weyl, Naturwissenschaften 19, 49 (1931).

${ }^{54}$ P. A. M. Dirac, Proc. R. Soc. A 333, 403 (1973).

${ }^{55}$ M. Omote and M. Kasuya, Prog. Theo. Phys. 58, 1627 (1977).

${ }^{56}$ D. Šijački, Phys. Lett. B 109, 435 (1982).

57 Y. Ne'eman and D. Šijački, Phys. Lett. B 200, 489 (1988).

${ }^{58}$ V. Canuto, P. J. Adams, S. H. Hsieh, and E. Tsiang, Phys. Rev. D 16, 1643 (1977).

${ }^{59}$ S. L. Adler, Rev. Mod. Phys. 54, 729 (1982).

${ }^{60}$ A. Zee, Ann. Phys. 151, 431 (1983).

${ }^{61}$ I. Antoniadis, J. Iliopoulos, and T. Tomaras, Nucl. Phys. B 261, 157 (1985).

${ }^{62}$ P. D. Mannheim and D. Kazanas, Astrophys. J. 342, 635 (1989).

${ }^{63}$ To keep the notation simple, however, we will still indicate Lorentz transformations by $\Lambda$, rather than $S L(2, C)$ elements.

${ }^{64} \mathrm{~A}$ re-expression of the Dirac Lagrangian is presented in Appendix A, which lends itself to the modelling of classical point matter particles, which we will discuss in Section II M.

${ }^{65}$ It is worth noting that the form adopted for the $(\Lambda, \rho)$-covariant derivative $D_{\mu}^{*} \varphi$ in (20) constitutes a choice about how to include the rotational and dilational gauge fields $A^{a b}{ }_{\mu}$ and $B_{\mu}$, respectively, which leads directly to the requirements (23) and (24) on their transformation properties.

${ }^{66}$ It is worth noting that, under a local dilation alone, if the field $\varphi$ has weight $w$, then $\mathcal{D}_{\mu}^{*} \varphi$ also transforms covariantly with weight $w$, whereas $\mathcal{D}_{a}^{*} \varphi$ has weight $w-1$.

${ }^{67}$ Since $\boldsymbol{h}$ is not symmetric, placing $\boldsymbol{J}$ into its first "slot" instead will, in general, lead to a new vector field that is different from $\mathcal{J}$, so that, for example, $h^{\mu}{ }_{a} J_{\mu} \neq \mathcal{J}_{a}$. We will not, however, need to consider this eventuality and may restrict our attention to the case in which the first index on the components of $\boldsymbol{h}$ (and $\boldsymbol{b}$ ) is Latin and the second is Greek.

${ }^{68}$ Unfortunately, this presents us with a notational difficulty. For objects possessing several indices, one should, in principle, use a different kernel symbol for each possible combination of Latin and Greek indices, but such an approach would quickly become unwieldy. So, in general, we will simply retain the original kernel letter, while keeping the above observations in mind. As a compromise, however, we will adopt the notation used above, which is in keeping with the definition (29), that the kernel letter of a quantity possessing only Latin indices (and its contractions over such indices) is the calligraphic font version of the kernel letter of the quantity possessing only Greek indices (following Lasenby, Doran and Gull), with the exception of quantities having Greek or lower-case kernel letters.

${ }^{69}$ It is also worth pointing out that, in terms of their counterparts ${ }^{0} \mathcal{A}_{a b c}$ and $\mathcal{K}_{a b c}$ in PGT (see Appendix B), one has ${ }^{0} \mathcal{A}^{* a b}{ }_{c}={ }^{0} \mathcal{A}^{a b}{ }_{c}+\delta_{c}^{a} \mathcal{B}^{b}-\delta_{c}^{b} \mathcal{B}^{a}$ and $\mathcal{K}^{* a b}{ }_{c}=\mathcal{K}^{a b}{ }_{c}-\delta_{c}^{a} \mathcal{B}^{b}+\delta_{c}^{b} \mathcal{B}^{a}$, so that the decomposition (46) or (47) also holds with all asterisks removed from the RHS.

${ }^{70}$ It is worth noting that the PGT (and WGT) rotational gauge field strength tensor (or "curvature") $\mathcal{R}_{a b c d}$ is already covariant under local dilations, whereas the PGT translational gauge field strength (or "torsion") $\mathcal{T}_{a b c}$ is not; one must instead introduce the WGT "torsion" (44).

${ }^{71}$ H. T. Nieh, J. Math. Phys. 21, 1439 (1980).

${ }^{72}$ One could alternatively define $t^{a}{ }_{\mu} \equiv h \delta \mathcal{L}_{\mathrm{G}} / \delta h_{a}{ }^{\mu}, s_{a b}{ }^{\mu} \equiv h \delta \mathcal{L}_{\mathrm{G}} / \delta A^{a b}{ }_{\mu}$, and $j^{\mu} \equiv h \delta \mathcal{L}_{\mathrm{G}} / \delta B_{\mu}$, and similarly for the matter sector. This choice corresponds more closely to the standard "metrical" versions of such tensors, but we will not adopt this convention here. 
${ }^{73}$ We note that, slightly unusually, in the last two definitions it is not the $h$-field, but its inverse, that is used to convert a Greek index into a Roman one; this should be borne in mind when dealing with these quantities.

${ }^{74}$ A. Saa, Gravitation Cosmol. 2, 89 (1996).

${ }^{75}$ To include gravitational effects in the action (A8) for a spinning point particle, one must, in addition, make the replacement $\dot{R} \equiv \dot{x}^{\mu} \partial_{\mu} R \rightarrow \dot{v}^{a} \mathcal{D}_{a}^{*} R$.

${ }^{76}$ It is worth noting that this form for the equation of motion of a test particle appears naturally in scalar-tensor gravity theory. ${ }^{77,78}$ In the standard interpretation, the scalar field in scalar-tensor gravity in fact plays the same role of introducing a physical scale, in a similar manner to the scalar field in the WGT, although see Section II L for a further discussion of this issue.

${ }^{77}$ C. Brans and R. H. Dicke, Phys. Rev. 124, 925 (1961).

${ }^{78}$ D. Puetzfeld and Y. N. Obukhov, Phys. Rev. D 92, 081502 (2015).

${ }^{79}$ N. Rosen, Found. Phys. 12, 213 (1982).

${ }^{80}$ S. Mirabotalebi, S. Jalalzadeh, S. Movahed, and H. R. Sepangi, Mon. Not. R. Astron. Soc. 385, 986 (2008).

${ }^{81}$ It is easily shown that $\bar{\psi} \gamma^{a 0} \stackrel{\leftrightarrow}{\mathcal{D}}_{a}^{*} \psi=\bar{\psi} \gamma^{a 0} \stackrel{\leftrightarrow}{\mathcal{D}}_{a} \psi$ (which does not depend on the dilation gauge field $B_{\mu}$ ), so the kinetic term in the Dirac action in reduced PGT is already invariant under local dilations, in the same way as its counterpart in "full" PGT, as discussed in Section II J.

${ }^{82}$ Q. Exirifard and M. M. Sheikh-Jabbari, Phys. Lett. B 661, 158 (2008).

${ }^{83}$ M. Borunda, B. Janssen, and M. Bastero-Gil, J. Cosmol. Astropart. Phys. 11, 008 (2008).

${ }^{84}$ N. Dadhich and J. M. Pons, Phys. Lett. B 705, 139 (2011).

${ }^{85}$ Since the $\boldsymbol{e}_{\mu}(x)$ are derived from a coordinate system, they form a holonomic set of basis vectors, whereas the set $\hat{\boldsymbol{e}}_{\boldsymbol{a}}(x)$ is, in general, non-holonomic.

${ }^{86}$ We need not consider the local Lorentz and GCT parts of the transformation, since the behaviour of the gravitational gauge fields $h_{a}{ }^{\mu}$ and $A^{a b}{ }_{\mu}$ under these transformations will remain the same as in WGT.

${ }^{87}$ The arbitrary parameter $\theta$ could, in principle, be promoted to a field $\theta(x)$. Provided it has Weyl weight $w=0$, one would obtain the same $\theta$-independent form for the eWGT covariant derivative $D_{\mu}^{\dagger}$ and the same gauge theory.

${ }^{88}$ The dagger on the derivative operator is simply to distinguish the eWGT covariant derivative from the PGT and WGT covariant derivatives $D_{\mu}$ and $D_{\mu}^{*}$, respectively, and should not be confused with the operation of Hermitian conjugation. In particular, we note that the eWGT covariant derivative $D_{\mu}^{\dagger} \varphi=\left[D_{\mu}+\frac{1}{2}\left(\mathcal{V}^{a} b^{b}{ }_{\mu}-\mathcal{V}^{b} b^{a}{ }_{\mu}\right) \Sigma_{a b}-w V_{\mu}-\frac{1}{3} w T_{\mu}\right] \varphi$ represents a far more substantial modification to $D_{\mu} \varphi$ than the WGT covariant derivative $D_{\mu}^{*} \varphi=\left(D_{\mu}+w B_{\mu}\right) \varphi$.

${ }^{89}$ Y. N. Obukhov, Phys. Lett. A 90, 13 (1982).

${ }^{90}$ M. Kaku, P. K. Townsend, and P. Van Nieuwenhuizen, Phys. Lett. B 69, 304 (1977).

${ }^{91}$ J. T. Wheeler, Phys. Rev. D 44, 1769 (1991).

${ }^{92}$ In terms of their counterparts ${ }^{0} \mathcal{A}_{a b c}$ and $\mathcal{K}_{a b c}$ in PGT (see Appendix B), one has ${ }^{0} \mathcal{A}^{\dagger a b}{ }_{c}={ }^{0} \mathcal{A}^{a b}{ }_{c}-\delta_{c}^{a}\left(\mathcal{V}^{b}+\right.$ $\left.\frac{1}{3} \mathcal{T}^{b}\right)+\delta_{c}^{b}\left(\mathcal{V}^{a}+\frac{1}{3} \mathcal{T}^{a}\right)$ and $\mathcal{K}^{\dagger a b}{ }_{c}=\mathcal{K}^{a b}{ }_{c}+\frac{1}{3} \delta_{c}^{a} \mathcal{T}^{b}-\frac{1}{3} \delta_{c}^{b} \mathcal{T}^{a}$, so confirming that the decomposition (158) also holds with all daggers removed, as discussed in Section II E.

${ }^{93}$ An alternative and more "automated" route for constructing covariant forms of $t_{a b}$ and $\tau_{a b}$ is given in Section III J.

${ }^{94}$ It is easily shown that $\bar{\psi} \gamma^{a 0} \stackrel{\leftrightarrow}{\mathcal{D}_{a}^{\dagger}} \psi=\bar{\psi} \gamma^{a 0} \stackrel{\leftrightarrow}{\mathcal{D}_{a}} \psi$ (which does not depend on the dilation gauge field $V_{\mu}$ ), so the kinetic term in the Dirac action in reduced PGT is already invariant under extended local dilations, in the same way as its counterpart in "full" PGT, as discussed in Section III K.

${ }^{95}$ It is worth noting that the semi-metricity condition (211) and that given in (124) for WGT can both be written as $\nabla_{\sigma} g_{\mu \nu}=-\frac{1}{2}\left(\Gamma_{\lambda \sigma}^{\lambda}-{ }^{0} \Gamma_{\lambda \sigma}^{\lambda}\right) g_{\mu \nu}$, where ${ }^{0} \Gamma_{\mu \nu}^{\lambda}$ is the metric connection; this also holds in PGT.

${ }^{96}$ One can straightforwardly construct parity-odd terms for inclusion in the free gravitational Lagrangian in an analogous manner to WGT, but we do not consider such terms here.

${ }^{97}$ The expression (222) can also be written in a form for which its symmetries are more manifest, namely, $L_{\mathcal{R}^{2}}=$ $\alpha_{1} \mathcal{R}^{\dagger 2}+\alpha_{2}^{\prime} \mathcal{R}_{(a b)}^{\dagger} \mathcal{R}^{\dagger(a b)}+\alpha_{3}^{\prime} \mathcal{R}_{[a b]}^{\dagger} \mathcal{R}^{\dagger[a b]}+\alpha_{4}^{\prime} \mathcal{R}_{a(b c) d}^{\dagger} \mathcal{R}^{\dagger a(b c) d}+\alpha_{5}^{\prime} \mathcal{R}_{a[b c] d}^{\dagger} \mathcal{R}^{\dagger a[b c] d}+\alpha_{6} \mathcal{R}_{a b c d}^{\dagger} \mathcal{R}^{\dagger c d a b}$, where the new coefficients are $\alpha_{2}^{\prime}=\alpha_{2}+\alpha_{3}, \alpha_{3}^{\prime}=\alpha_{2}-\alpha_{3}, \alpha_{4}^{\prime}=\alpha_{4}+\alpha_{5}$, and $\alpha_{5}^{\prime}=\alpha_{4}-\alpha_{5}$. In this case, the eWGT version of the Gauss-Bonnet identity allows one to set $\alpha_{1}$ or $\alpha_{6}$ to zero as before, or set $\alpha_{2}^{\prime}=\alpha_{3}^{\prime}$.

${ }^{98}$ The expression (227) can also be written in the more manifestly symmetric form $L_{\mathcal{T}^{2}}=\beta_{1}^{\prime} \mathcal{T}_{(a b) c}^{\dagger} \mathcal{T}^{\dagger(a b) c}+$ $\beta_{2}^{\prime} \mathcal{T}_{[a b] c}^{\dagger} \mathcal{T}^{\dagger[a b] c}$, where $\beta_{1}^{\prime}=\beta_{1}+\beta_{2}$ and $\beta_{2}^{\prime}=\beta_{1}-\beta_{2}$.

${ }^{99}$ One might even consider the case in which $S_{\mathrm{G}}$ and $S_{\mathrm{M}}$ are themselves not individually invariant under (extended) local dilations, but the total action $S_{\mathrm{T}}=S_{\mathrm{G}}+S_{\mathrm{M}}$ is so. We will not pursue this class of theories, however, since they would require the presence of (non locally scale-invariant) matter to induce local scale invariance of the full theory in the presence of gravitation (although such a possibility cannot be ruled out a priori). It seems more natural to demand the theory to be scale invariant in vacuo, in which case $S_{\mathrm{G}}$ and $S_{\mathrm{M}}$ must be separately locally scale invariant.

100 One may also straightforwardly include the electromagnetic field in $\mathcal{L}_{\mathrm{M}}$, coupled both to the Dirac field and to gravity, in an analogous manner to that discussed at the end of Section II K, but will we not consider this extension here.

101 P. D. Mannheim, Prog. Part. Nucl. Phys. 56, 340 (2006).

102 C. M. Bender and P. D. Mannheim, Phys. Rev. Lett. 100, 110402 (2008).

103 Once again, one may also straightforwardly include the electromagnetic field in $\mathcal{L}_{\mathrm{M}}$, coupled both to the Dirac field and to gravity, but will we not consider this extension here.

${ }^{104}$ C. Doran, A. N. Lasenby, A. Challinor, and S. F. Gull, J. Math. Phys. 39, 3303 (1998).

${ }^{105}$ Moreover, after a long but straightforward calculation, one may show that ${ }^{0} \mathcal{R}^{a b}{ }_{c d}$ is itself related to the corresponding WGT quantity ${ }^{0} \mathcal{R}^{* a b}{ }_{c d}$ by ${ }^{0} \mathcal{R}^{* a b}{ }_{c d}={ }^{0} \mathcal{R}^{a b}{ }_{c d}-2 \delta_{d}^{[b}\left({ }^{0} \mathcal{D}_{c}-\mathcal{B}_{c}\right) \mathcal{B}^{a]}+2 \delta_{c}^{[b}\left({ }^{0} \mathcal{D}_{d}-\mathcal{B}_{d}\right) \mathcal{B}^{a]}-2 \mathcal{B}^{e} \mathcal{B}_{e} \delta_{c}^{[a} \delta_{d}^{b]}$, from which one finds that their contractions are related by ${ }^{0} \mathcal{R}^{* a}{ }_{c}={ }^{0} \mathcal{R}^{a}{ }_{c}-2\left({ }^{0} \mathcal{D}_{c}-\mathcal{B}_{c}\right) \mathcal{B}^{a}-\left({ }^{0} \mathcal{D}_{e}+2 \mathcal{B}_{e}\right) \mathcal{B}^{e} \delta_{c}^{a}$ and 
${ }^{0} \mathcal{R}^{*}={ }^{0} \mathcal{R}-6\left({ }^{0} \mathcal{D}_{e}+\mathcal{B}_{e}\right) \mathcal{B}^{e}$. Similarly, ${ }^{0} \mathcal{R}^{a b}{ }_{c d}$ and its contractions are related to the corresponding eWGT quantities by the same relationships, but with the replacements ${ }^{0} \mathcal{R}^{* a b}{ }_{c d} \rightarrow{ }^{0} \mathcal{R}^{\dagger a b}{ }_{c d}$ and $\mathcal{B}_{a} \rightarrow-\left(\mathcal{V}_{a}+\frac{1}{3} \mathcal{T}_{a}\right)$.

${ }^{106}$ More appropriately, the theory is also known as Einstein-Cartan-Kibble-Sciama (ECKS) theory, but we shall adopt the more usual, shorter naming convention (with apologies to Kibble and Sciama).

${ }^{107}$ A. Lewis, A. N. Lasenby et al., "Scale invariant gauge theory gravity," http://cosmologist.info/notes/old/ConfGrav.ps (unpublished).

${ }^{108}$ It is probably fair to say that Dirac would not have considered $L_{\mathrm{G}}$ in (D1) to be his entire gravitational Lagrangian and would have also included the term proportional to $\phi^{2} \mathcal{R}$ that appears in (D2). Since this term contains the scalar field $\phi$, however, we have instead included it in the matter Lagrangian.

${ }^{109}$ In fact, Dirac's original theory has vanishing torsion and so he proposed a matter Lagrangian of the form (D2), but with the WGT covariant derivative and Ricci scalar replaced by their "reduced" versions ${ }^{0} \mathcal{D}_{a}^{*}$ and ${ }^{0} \mathcal{R}^{*}$, respectively, as described in Section II E. We extend his approach here to include torsion. 